Universidade de São Paulo

Escola Politécnica da USP

RICARDO DOLL LAHUERTA

\title{
Projeto de Mecanismos Flexíveis Baseado no Efeito da Flambagem Não Linear Utilizando o
} Método de Otimização Topológica 



\section{Projeto de Mecanismos Flexíveis Baseado no Efeito da Flambagem Não Linear Utilizando o Método de Otimização Topológica}

Tese apresentada à Escola Politécnica da Universidade de São Paulo para obtenção do Título de Doutor em Ciências.

Área de Concentração:

Engenharia de Controle e Automação Mecânica.

Orientador:

Prof. Dr. Emílio Carlos Nelli Silva

São Paulo 

Este exemplar foi revisado e corrigido em relação à versão original, sob responsabilidade única do autor e com a anuência de seu orientador.

São Paulo, de de

Assinatura do autor:

Assinatura do orientador:

Catalogação-na-publicação

Doll Lahuerta, Ricardo

Projeto de Mecanismos Flexíveis Baseado no Efeito da Flambagem Não Linear Utilizando o Método de Otimização Topológica / R. Doll Lahuerta -versão corr. -- São Paulo, 2017.

$122 \mathrm{p}$.

Tese (Doutorado) - Escola Politécnica da Universidade de São Paulo. Departamento de Engenharia Mecatrônica e de Sistemas Mecânicos.

1.Método de Otimização Topológica 2.Método de Elementos Finitos 3.Mecanismos Flexíveis 4.Flambagem Não Linear 5.Policonvexidade I.Universidade de São Paulo. Escola Politécnica. Departamento de Engenharia Mecatrônica e de Sistemas Mecânicos II.t. 



\section{Dedicatória}

Aos meus pais, Leonardo e Marli, que sempre incentivaram a busca pelo conhecimento e aos meus queridos irmãos Luiz Fernando e Rafael pelo apoio em todos os momentos.

À minha amada esposa pelo apoio incondicional na realização dos meus sonhos. E ao meu querido filho por me trazer a alegria e incentivo que eu precisava para a conclusão deste projeto. Muito Obrigado! 



\section{Agradecimentos}

À minha família;

Aos amigos;

Ao meu orientador;

À CAPES, pelo apoio financeiro;

À USP pela oportunidade. 

"Sucesso. O sucesso é ir de fracasso em fracasso sem perder entusiasmo." Winston Churchill

"A imaginação é mais importante que o conhecimento."

Albert Einstein

"Sonhos não morrem, apenas adormecem na alma da gente."

Chico Xavier 



\section{Resumo}

Mecanismo Flexível é um dispositivo mecânico utilizado para transformar movimento, força ou energia entre as portas de entrada e saída sem a presença de juntas, pinos baseados em uma estrutura em monolítica, em outras palavras, a transformação do movimento é dada pela flexibilidade de sua estrutura. Deste modo a transformação pode ser direcionada em uma direção em específico, amplificando ou reduzindo o deslocamento ou força aplicados. Por este motivo mecanismos flexíveis tem grandes aplicações em micromanipulação e nano posicionamento. A concepção deste tipo de mecanismo é complexa e uma das possibilidades de elaboração deste dispositivo mecânico é através da distribuição de flexibilidade ou rigidez dentro do domínio de projeto utilizando o Método de Otimização Topológica (MOT), que essencialmente combina algoritmos de otimização numéricos com o Método de Elementos Finitos (MEF), por exemplo. A grande maioria das classes de mecanismos flexíveis existentes trabalha sob pequenos deslocamentos, na ordem de micro ou nano metros, no entanto, existe uma classe de mecanismos que utiliza o recurso da flambagem não linear para operar com grandes deslocamentos. O procedimento de concepção desta de classe de mecanismo é complexa e ainda se encontra em estagio inicial, necessitando de aprimoramentos que permitam o seu projeto completo via métodos computacionais. Portanto, esta tese foi desenvolvida como objetivo desenvolver uma metodologia computacional para projetar esta classe de mecanismo flexível inovador que emprega a flambagem não linear na sua estrutura como meio para obter sob grandes deslocamentos na porta de saída. A metodologia desenvolvida se baseia no MOT para obter a topologia da estrutura que satisfaça as restrições de projeto. A modelagem do comportamento físico da estrutura utiliza uma formulação variacional não linear do problema elástico, considerando a cinemática 
não linear com um modelo constitutivo policonvexo. $\mathrm{O}$ modelo de material aplicado para obter a topologia da estrutura do mecanismo foi o Solid Isotropic Material with Penalization (SIMP) com um algoritmo de otimização numérico baseado no método de ponto interior, onde foi utilizada a implementação do [pOpt em conjunto com a plataforma Python FEniCS de soluções de Equações Diferenciais Parciais (EDPs). São apresentados resultados bidimensionais de mecanismos considerando algumas configurações de geometria, condições de contorno e restrições de flambagem não-linear, como incremento de carga.

Palavras-chave: Mecanismos Flexíveis. Policonvexidade. Método de Elementos Finitos. Flambagem Não Linear. Método de Otimização Topológica. Método de Ponto Interno. 


\section{Abstract}

The compliant mechanism is a mechanical device used to transform displacement, force or energy between the input and output ports without joints, pins based on a monolithic structure, in other words, the motion transformation is given by the flexibility of its structure. In this way the movement can be defined to a specific axis direction, amplifying or reducing the applied displacement or force. For this reason, the compliant mechanism has significant applications in micromanipulation and nanopositioning system. The design of this type of device is intricate, and one way to achieve such design is trying to distribution flexibility or rigidity within the design domain using the Topology Optimization Method (TOM), which essentially combines numerical optimization algorithms with Finite Element Method ( $\overline{\text { FEM) }}$, for example. Most models of existing compliant mechanism work under small displacements, in the order of micro or nanometers, nevertheless, there is a class of such mechanisms that uses the nonlinear buckling behavior to operate under large displacements. The design process of this mechanism type is complicated and is still at early stages, requiring improvements that allow a complete design process via computational methods. Therefore, this thesis goal is to develop a computational methodology to create this class of innovative compliant mechanism that employs nonlinear buckling behavior to work under large displacement at the output port. The approach developed is based on TOM to achieve the optimal structure topology that satisfies the design and optimization constraints. The modeling of the elasticity behavior of the structure relies on the nonlinear variational formulation, applying the nonlinear kinematics with a polyconvex constitutive 
model. The SIMP is employed as a material model to obtain the optimal topology of the mechanism structure with a numeric optimization algorithm based on the interior point method, where the IpOptimplementation was used with the high-level Python interfaces to FEniCS to solve the partial differential equations (표 $)$ problem. Two-dimensional results of mechanisms are presented considering some geometric, boundary configuration, and including nonlinear buckling as design constraints.

Keywords: Compliant Mechanism. Policonvexity. Finite Element Method. Nonlinear Buckling. Topology Optimization Method. Interior Point Method. 


\section{Lista de Abreviaturas}

CAD Computer-Aided Design

CAE Computer-Aided Engineering

MH Método de Homogeneização

MOT Método de Otimização Topológica

OT Otimização Topológica

TOM Topology Optimization Method

SIMP Solid Isotropic Material with Penalization

PVM Princípio do Variacional Misto

MEF Método de Elementos Finitos

FEM Finite Element Method

PK1 Primeiro Tensor das Tensões de Piola Kirchhoff

PK2 Segundo Tensor das Tensões de Piola Kirchhoff

KSV Lei Material Kirchhof Saint Venant

nH Lei Material neo-Hookiana de Simo-Ciarlet

PZT Titanato Zircanato de Chumbo 
MPI Método do Ponto Interior

IpOpt Interior-Point Optimization Algorithm

MAM Método das Assíntotas Móveis

MMQ Método dos Mínimos Quadrados

LSQ Least Squares Method

CAEM Coeficiente de Acoplamento Eletro-Mecânico

MEMS Microelectromechanical Systems

NEMS Nanoelectromechanical Systems

MA Mechanical Advantage

GA Geometrical Advantage

co Critério da Optimalidade

CONLIN Convex Linearization

MCGD Método do Controle Generalizado dos Deslocamentos

MCA Método do Comprimento de Arco

PGR Parâmetro Generalizado de Rigidez

MCA Método do Comprimento de Arco

PLS Programação Linear Sequencial

PQS Programação Quadrática Sequencial

PNL Programação Não-Linear

KKT Condição Karush-Kuhn-Tucker

EDP Equação Diferencial Parcial

EDPs Equações Diferenciais Parciais 
PDEs partial differential equations

EPD Estado Plano de Deformação

EPT Estado Plano de Tensão

FEniCS Finite Element Computational Software

UFL Unified Form Language

FFC FEniCS Form Compiler

LAPACK Linear Algebra Package 



\section{Lista de Símbolos}

\section{Operadores Algébricos}

\begin{tabular}{cl}
\hline Símbolos & Descrição \\
\hline $\boldsymbol{a} \cdot \boldsymbol{b}$ & Produto duplo (contração tensorial) \\
$\boldsymbol{a} \circ \boldsymbol{b}$ & Produto interno entre os vetores $\boldsymbol{a}$ e $\boldsymbol{b}$ \\
$\boldsymbol{a} \oslash \boldsymbol{b}$ & Divisão de Hadamard entre os vetores $\boldsymbol{a}$ e $\boldsymbol{b}$ \\
$\times$ & Produto vetorial \\
$\otimes$ & Produto diádico ou produto tensorial \\
$\nabla$ & Operador gradiente \\
\|\|$_{1}$ & Norma Euclidiana $L^{1}$ \\
\|\|$_{2}$ & Norma Euclidiana $L^{2}$ \\
$\operatorname{det}$ & Operador determinante \\
$\epsilon_{i j k}$ & Operador de permutação Levi-Civita \\
cof & Cofator \\
adj & Operador Adjunto \\
div & Operador divergente \\
$\operatorname{tr}$ & Operador traço \\
$(\bullet)^{\top}$ & Operador transposto \\
Sym & Operador de Simetria, Sym $(A)=\frac{1}{2}\left(A+A^{\top}\right)$ \\
Skew & Operador Anti-Simétrico, Skew $(A)=\frac{1}{2}\left(A-A^{\top}\right)$ \\
$\ln$ & Logaritmo natural \\
sign & Sinal de uma variável ou função \\
$\delta_{i j}$ & Deltade Kronecker \\
$(\mathrm{G} * \mathrm{a})$ & Operador de Convolução ou Integral de Convolução \\
\hline &
\end{tabular}




\section{Mecânica do Contínuo}

\begin{tabular}{cl}
\hline Símbolos & Descrição \\
\hline $\boldsymbol{x}^{\mathrm{r}}$ & Vetor de um ponto material na configuração de referência \\
$\boldsymbol{x}$ & Vetor de um ponto material na configuração atualizada \\
$\boldsymbol{u}$ & Vetor dos deslocamentos de um ponto material \\
$\boldsymbol{u}$ & Vetor de velocidade de um ponto material \\
$\ddot{\boldsymbol{u}}$ & Vetor de aceleração de um ponto material \\
$\boldsymbol{e}$ & Vetor de base na configuração atualizada \\
$\boldsymbol{e}^{\mathrm{r}}$ & Vetor de base na configuração referência \\
$\boldsymbol{n}$ & Vetor do plano normal na configuração atualizada \\
$\boldsymbol{n}^{\mathrm{r}}$ & Vetor do plano normal na configuração referência \\
$\boldsymbol{b}$ & Vetor das forças de volume na configuração atualizada \\
$\boldsymbol{b}^{\mathrm{r}}$ & Vetor das forças de volume na configuração referência \\
$\boldsymbol{t}^{\mathrm{r}}$ & Vetor das forças na superfície na configuração atualizada \\
$\boldsymbol{t}^{\mathrm{r}}$ & Vetor das forças na superfície na configuração referência \\
$n$ & Dimensão do problema, 2 para 2Ds e 3 para 3Ds \\
$m$ & Massa específica na configuração atualizada \\
$m^{r}$ & Massa específica na configuração referência \\
$\boldsymbol{I}$ & Tensor identidade de segunda Ordem \\
$\boldsymbol{L}$ & Tensor do gradiente dos deslocamentos \\
$\boldsymbol{F}$ & Tensor do gradiente da transformação \\
$\boldsymbol{R}$ & Tensor de rotação \\
$\boldsymbol{f}_{\mathrm{i}}$ & Componente $i$ do tensor $\boldsymbol{F}$ (ver eq. 2.2) \\
$\boldsymbol{C}$ & Tensor direito de Cauchy-Green \\
$\boldsymbol{B}$ & Tensor esquerdo de Cauchy-Green \\
$\boldsymbol{E}$ & Tensor das deformações de Green \\
$\boldsymbol{P}$ & Primeiro tensor das tensões de Piola-Kirchhoff \\
$\boldsymbol{S}$ & Segundo tensor das tensões de Piola-Kirchhoff \\
$\boldsymbol{T}$ & Segundo tensor das tensões de Cauchy \\
$\boldsymbol{\tau}_{\boldsymbol{i}}$ & Componente $i$ do tensor $\boldsymbol{P}$ (ver eq. 2.22) \\
$\mathrm{I}_{1}^{\mathrm{C}}, \boldsymbol{I}_{2}^{\mathrm{C}}$ & Primeiro e segundo invariante do tensor direito de Cauchy (ver eq. \\
$\mathrm{I}_{1}^{\mathrm{E}}, \mathrm{I}_{2}^{\mathrm{E}}$ & Primeiro e segundo invariante do tensor de Green (ver eq. .2 $)$ \\
\hline &
\end{tabular}




\section{Mecânica do Contínuo}

\begin{tabular}{cl}
\hline Símbolos & Descrição \\
\hline $\mathcal{H}^{1}$ & Espaço de Hilbert \\
$\mathrm{U}_{\text {int }}$ & Energia potencial interna \\
$\mathrm{U}_{\text {ext }}$ & Energia potencial externa \\
$J$ & Determinante de $\boldsymbol{F}$ \\
$C_{i j}$ & Tensor tangente material em $\boldsymbol{P}: \boldsymbol{F}$ \\
$\boldsymbol{L}_{\mathrm{s}}$ & Tensor tangente das forças de superfície \\
$\boldsymbol{L}_{v}$ & Tensor tangente das forças de volume \\
$\varphi$ & Função de energia específica \\
$\boldsymbol{\omega}_{\alpha}^{0}$ & Tensor corretor de PK1] no EPT (eq. [13) \\
$\boldsymbol{Q}_{\alpha \beta}$ & Tensor corretor do tensor tangente material $C_{i j}$ no EPT] (eq. [.15) \\
$\lambda, \mu$ & Constantes de Lamé \\
$d a^{r}$ & Derivada da área na configuração de referência \\
$d v^{r}$ & Derivada do volume na configuração de referência \\
$\mathrm{V}^{r}$ & Volume na configuração de referência \\
\hline
\end{tabular}

\section{Implementação Numérica}

\begin{tabular}{cl}
\hline Símbolos & Descrição \\
\hline $\mathbf{N}_{e}$ & Matriz das funções de forma de um elemento e \\
$\mathbf{N}_{e, i}$ & Matriz das derivadas das funções de forma de um elemento e na \\
& direção $i$ \\
$\mathbf{f}_{\text {int }}$ & Vetor das força internas elásticas \\
$\mathbf{f}_{\text {ext }}$ & Vetor das força externas elásticas \\
$\mathbf{r}$ & Vetor dos resíduos \\
$\bar{\lambda}$ & Incremento de carga \\
$\overline{\Delta \lambda}$ & Fator de carga proporcional \\
$\mathbf{h}_{i}$ & Vetor de seleção dos graus de liberdade $i$ \\
$\mathbf{K}_{M}$ & Matriz tangente material \\
$\mathbf{K}_{V}$ & Matriz tangente das forças de volume \\
$\mathbf{K}_{S}$ & Matriz tangente das forças de superfície \\
$\mathbf{K}_{\mathrm{T}}$ & Matriz tangente \\
\hline
\end{tabular}




\begin{tabular}{cl}
\hline & \multicolumn{1}{c}{ Otimização } \\
\hline Símbolos & Descrição \\
\hline $\mathcal{L}$ & Função Lagrangiana \\
$\Lambda_{j}$ & Multiplicadores de Lagrange ou variáveis duais \\
$b_{j}$ & Valores das restrições Lagrangianas \\
$n_{g}$ & Número de restrições \\
$\mu_{\mathrm{o}}$ & Parâmetro de barreira \\
$B\left(\mu_{\mathrm{o}}\right)$ & Função de barreira \\
$S_{n}$ & Função de mínimos quadrados \\
$w_{n}$ & Parâmetro de penalização da função de mínimos quadrados \\
$\bar{\Omega}_{e}^{r}$ & Domínio da função de projeção ao redor do elemento (e) na \\
$r_{\text {min }}$ & configuração de referência \\
$\overline{\mathbf{w}}_{e}$ & Raio de projeção (figura 4.5) \\
& Vetor com os pesos da função de projeção referente ao elemento (e) \\
\hline
\end{tabular}

Atuadores/ Mecanismo Flexível

\begin{tabular}{cl}
\hline Símbolos & Descrição \\
\hline$\partial \Omega_{\text {in }}^{r}$ & Área da porta de entrada \\
$\partial \Omega_{\mathrm{ou}}^{\mathrm{r}}$ & Área da porta de saída \\
$W_{\mathrm{in}}$ & Energia na porta de entrada (eq. 4.25$)$ \\
$W_{\mathrm{ou}}$ & Energia na porta de saída (eq. 4.25$)$ \\
$\mathbf{f}_{\mathrm{in}}$ & Vetor de força na porta de entrada \\
$\mathbf{f}_{\mathrm{ou}}$ & Vetor de força de saída \\
$\mathbf{u}_{\mathrm{in}}$ & Vetor dos deslocamentos na porta de entrada \\
$\mathbf{u}_{\mathrm{ou}}$ & Vetor dos deslocamentos na porta de saída \\
$U$ & Energia interna retida pela estrutura ou mecanismo \\
$\mathbf{U}_{\mathrm{ext}}$ & Função de flexibilidade externa (end compliance) \\
\hline
\end{tabular}




\section{Lista de Figuras}

1.1 Exemplos de Atuadores Flextensionais piezelétricos. . . . . . . . . . . ₹

1.2 Constituição básica de um transdutor piezocompósito. . . . . . . . . . 百

1.3 Configuração de trabalho do atuador piezelétrico que utiliza o mecanismo de flambagem não linear . . . . . . . . . . . . . . . . . . . 苗

1.4 Exemplo do processo iterativo do MOT . . . . . . . . . . . . 5

1.5 Procedimento de projeto de atuadores flextensionais que utilizam o mecanismo de flambagem não linear via o MOT, . . . . . . . . . . . . . . . 6

2.1 Configurações de um sólido deformável no espaço . . . . . . . . . . . . . . 20

3.1 Representação da hipótese doEPD $\left(\gamma_{33}=0\right.$ e $\left.\tau_{33} \neq 0\right) . \ldots \ldots$. . . . . . 38

3.2 Representação da hipótese doEPT $\left(\gamma_{33} \neq 0\right.$ e $\left.\tau_{33}=0\right) \ldots \ldots \ldots \ldots$

3.3 Representação da hipótese de axissimetria. . . . . . . . . . . . . 40

3.4 (a) Configuração do modelo do pórtico Lee. (b) Curva incremental de carga versus deslocamento da estrutura nos nós de aplicação.

3.5 Gráfico da Energia de Deformação Específica $\left(\varphi^{0}\right)$ em relação ao deslocamento e ao incremento de pseudo-tempo do modelo do pórtico Lee. . . . 53 
4.1 Topologia de um sólido deformável no MOT . . . . . . . . . . . . 61

4.2 Influência do fator de penalização na propriedade efetiva da função de energia de deformação específica. . . . . . . . . . . . . . . 63

4.3 Representação da escala de cinza. . . . . . . . . . . . . . . . . 65

4.4 Resultado com instabilidade de tabuleiro. . . . . . . . . . . . 65

4.5 Representação do raio de influência $r_{\text {min }}$ considerando a penalização a função de ponderação $\bar{w}_{e}^{p}$ onde $p=1 \ldots \ldots \ldots \ldots \ldots$

4.6 Resultados de topologia ótima para diferentes tamanhos de elementos para uma viga engastada de dimensões de $1000 \times 250 \mathrm{~mm}$, para $r_{\min }=30$ com uma discretização de 175, 1250, 2500 e 5000 elementos respectivamente. . . . . . . . . . . . . . . . . . 60

4.7 Representação do domínio de projeto de um mecanismo flexível. . . . . . 72

4.8 Generalização do algorítimo de Otimização Topológica (OT). . . . . . . . . 76

5.1 Configuração do domínio fixo estendido para à síntese de um mecanismo biestável. . . . . . . . . . . . . . . . . . 799

5.2 Topologia do mecanismo flexível biestável considerando a restrição de volume em $60 \% \ldots \ldots \ldots \ldots \ldots$

5.3 Evolução da função objetivo considerando a restrição de volume em $60 \%$. : 80

5.4 Gráfico de evolução das funções de restrições. . . . . . . . . . . . . . . 8̊0

5.5 Gráfico de incremento de carga em relação ao deslocamento (a) e pseudotempo (b) considerando a restrição de volume em $60 \%$. . . . . . . . . 8 81

5.6 Malha da interpretação dos resultados considerando a restrição de volume em $60 \%$. . . . . . . . . . . . . . . . . 81

5.7 Gráfico de incremento de carga em relação ao deslocamento (a) e pseudotempo (b), configuração deformada (c) do modelo interpretado considerando a restrição de volume em $60 \% \ldots \ldots \ldots \ldots \ldots \ldots$

5.8 Topologia do mecanismo flexível biestável considerando a restrição de volume em $50 \% \ldots \ldots \ldots \ldots \ldots \ldots$ 
5.9 Evolução da função objetivo considerando a restrição de volume em $50 \%$.

5.10 Gráfico de evolução das funções de restrições. . . . . . . . . . . . . . 883

5.11 Gráfico de incremento de carga em relação ao deslocamento (a) e pseudotempo (b) considerando a restrição de volume em $50 \%$. . . . . . . . . . 通

5.12 Malha da interpretação dos resultados considerando a restrição de volume em $50 \%$. . . . . . . . . . . . . . . . . . . . . . .

5.13 Gráfico de incremento de carga em relação ao deslocamento (a) e pseudotempo (b), configuração deformada (c) do modelo interpretado considerando a restrição de volume em $50 \% \ldots$. . . . . . . . . . . . . 8 8

5.14 Topologia do mecanismo flexível biestável considerando a restrição de volume em $40 \% \ldots \ldots \ldots \ldots \ldots$. . . . . . . . . . . . . 85

5.15 Evolução da função objetivo considerando a restrição de volume em $40 \%$.

86

5.16 Gráfico de evolução das funções de restrições. . . . . . . . . . . . . . . . 886

5.17 Gráfico de incremento de carga em relação ao deslocamento (a) e pseudotempo (b) considerando a restrição de volume em $40 \%$. . . . . . . . . 86

5.18 Malha da interpretação dos resultados considerando a restrição de volume em $40 \%$. . . . . . . . . . . . . . . . . . . . . . 878

5.19 Gráfico de incremento de carga em relação ao deslocamento (a) e pseudotempo (b), configuração deformada (c) do modelo interpretado considerando a restrição de volume em $40 \%$. . . . . . . . . . . . . . . 80

5.20 Topologia do mecanismo flexível biestável considerando a restrição de volume em $50 \% \ldots \ldots \ldots \ldots \ldots \ldots \ldots \ldots \ldots \ldots$. . . . . . . . . . . . . . .

5.21 Evolução da função objetivo considerando a restrição de volume em $50 \%$.

5.22 Gráfico de evolução das funções de restrições.

5.23 Gráfico de incremento de carga em relação ao deslocamento (a) e pseudotempo (b) considerando a restrição de volume em $50 \%$. . . . . . . . . . 898

5.24 Malha da interpretação dos resultados considerando a restrição de volume em $50 \%$. . . . . . . . . . . . . . . . . . . . . . . . 90 
5.25 Gráfico de incremento de carga em relação ao deslocamento (a) e pseudotempo (b), configuração deformada (c) do modelo interpretado considerando a restrição de volume em $50 \%$. . . . . . . . . . . . . . . . . . .

5.26 Configuração do domínio fixo estendido para à síntese de um mecanismo flexível inversor. . . . . . . . . . . . . . . . . . . . . 914

5.27 Topologia do mecanismo flexível biestável inversor considerando a restrição de volume em $40 \% \ldots \ldots \ldots \ldots . \ldots \ldots$

5.28 Evolução da função objetivo considerando a restrição de volume em $60 \%$. 93

5.29 Evolução das funções de restrições de incremento de carga e fração de volume. . . . . . . . . . . . . . . . . . . 94

5.30 Evolução da restrição de deslocamento em $\partial \Omega_{\mathrm{ou}}^{\mathrm{r}} \ldots \ldots \ldots \ldots$

5.31 Gráfico de incremento de carga em relação ao deslocamento (a) e pseudotempo (b) considerando a restrição de volume em $40 \%$. . . . . . . . . . 94

5.32 Malha da interpretação dos resultados para um mecanismo inversor considerando a restrição de volume em $40 \%$. . . . . . . . . . . 95

5.33 Gráfico de incremento de carga em relação ao deslocamento (a) e pseudotempo (b), configuração deformada (c) do modelo interpretado considerando a restrição de volume em $40 \%$. . . . . . . . . . . . . . .

5.34 Avaliação dos deslocamentos relativo ao resultado obtido para um mecanismo biestável inversor considerando a restrição de volume em 40\%. . . 96

6.1 Comparação entres os resultados da literatura com os resultados obtidos com a metodologia desenvolvida neste trabalho. . . . . . . . . . . 99 


\section{Lista de Tabelas}

5.1 Propriedade elásticas do material utilizado . . . . . . . . . . . . 77 



\section{Sumário}

1. Introdução $\ldots \ldots \ldots \ldots \ldots \ldots \ldots \ldots \ldots \ldots \ldots$. . . . . . . .

1.1 Projeto de Mecanismos Flexíveis considerando o efeito da flambagem não-linear via MOT . . . . . . . . . . . . . . . . . . . . 由

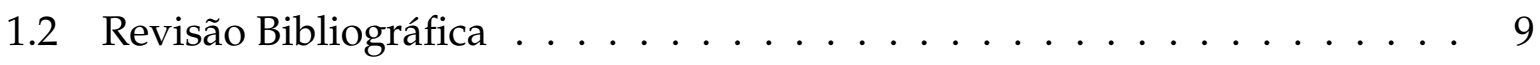

1.3 Objetivos ................... 14

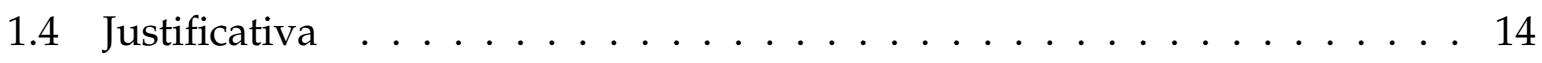

1.5 Contribuições Científicas . . . . . . . . . . . . . . . . . 15

1.6 Organização da Tese $\ldots \ldots \ldots \ldots \ldots \ldots \ldots \ldots$

1.7 Notação Matemática . . . . . . . . . . . . . . . . . . . . . . . 18

2. Formulação Teórica . . . . . . . . . . . . . . . . . . . . . . . . . 19

2.1 Mecânica do Contínuo . . . . . . . . . . . . . . . . 19

2.2 Equações Cinemáticas dos Sólidos Deformáveis $\ldots \ldots \ldots \ldots$

2.3 Princípio da Objetividade Material $\ldots \ldots \ldots \ldots \ldots$

2.4 Policonvexidade . . . . . . . . . . . . . . . . . . . . . 29 
2.5 Lei de Material de neo-Hookiano de Simo-Ciarlet

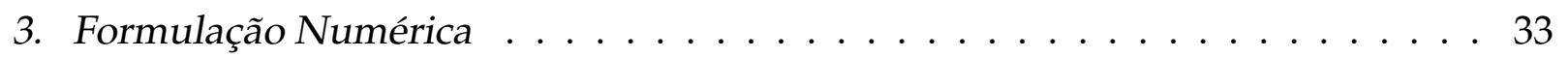

3.1 Ambiente de Implementação . . . . . . . . . . . . . . . . . . . 33

3.2 Linearização da Forma Fraca . . . . . . . . . . . . . . . . . 34

3.3 Método de Elementos Finitos . . . . . . . . . . . . . 35

3.4 Aproximações Bidimensionais . . . . . . . . . . . . . . . 38

3.4.1 Estado Plano de Deformação -EPD . . . . . . . . . . . . . 38

3.4.2 Estado Plano de Tensão -EPT . . . . . . . . . . . . . . . . 39

3.4.3 Condição de Axissimetria . . . . . . . . . . . . . . . 40

3.4.4 Tensor Tangente Material para Lei Material de neo-Hookiano de Simo-Ciarlet ................... . . 41

3.5 Métodos de Solução de Sistemas . . . . . . . . . . . . . . . . . 43

3.5.1 Método de Newton . . . . . . . . . . . . . . . . 43

3.5.2 Método de Newton Incremental . . . . . . . . . . . . . . . . . 44

3.5.3 Método do Comprimento de Arco - MCA . . . . . . . . . . 45

3.5.4 Método do Controle Generalizado dos Deslocamentos - MCGD . . . 48

3.6 Instabilidade Elástica . . . . . . . . . . . . . . . 49

3.7 Otimização Numérica . . . . . . . . . . . . . . . 54

3.8 Método de Ponto Interior $\ldots \ldots \ldots \ldots \ldots$

3.9 Método dos Mínimos Quadrados . . . . . . . . . . . . . . . 56

4. Método de Otimização Topológica . . . . . . . . . . . . . . . . . . 59 
4.1 Modelo de material - SIMP . . . . . . . . . . . . . . . . . . 61

4.2 Função de Continuação $\ldots \ldots \ldots \ldots \ldots \ldots \ldots$

4.3 Problemas associados à discretização no MOT . . . . . . . . . . . . . 6 64

4.4 Função de Projeção $\ldots \ldots \ldots$. . . . . . . . . . . . . . . . 65

4.5 Análise de Sensibilidade . . . . . . . . . . . . . . . . 68

4.5.1 Energia de Deformação Externa . . . . . . . . . . . . . 68

4.5.2 Deslocamento . . . . . . . . . . . . . . . .

4.5.3 Fator de Incremento de carga . . . . . . . . . . . . . . 70

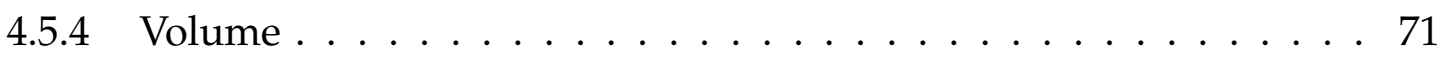

4.6 Sínteses de Mecanismos Flexíveis via Método de Otimização Topológica · 72

4.6.1 Minimização da Energia de Deformação Interna com Restrições via Método dos Mínimos Quadrados . . . . . . . . . . . . . 74

4.7 Implementação do MOT $\ldots \ldots \ldots \ldots \ldots \ldots \ldots$

5. Resultados ..................... 77

5.1 Mecanismo Flexível Biestável $\ldots \ldots \ldots \ldots \ldots$. . . . . . . 78

5.1 .1 Restrição de Volume em $60 \% \ldots \ldots \ldots \ldots$. . . . . . . . . .

5.1 .2 Restrição de Volume em $50 \% \ldots \ldots \ldots \ldots$. . . . . . . 82

5.1 .3 Restrição de Volume em $40 \% \ldots \ldots \ldots \ldots \ldots$

5.1.4 Restrição de Volume em 50\% com alteração na restrição de carga 87

5.2 Mecanismo Inversor $\ldots \ldots \ldots \ldots \ldots$

6. Conclusões . . . . . . . . . . . . . . . . . . . . . . 978 
6.1 Trabalhos Futuros . . . . . . . . . . . . . . . . 99

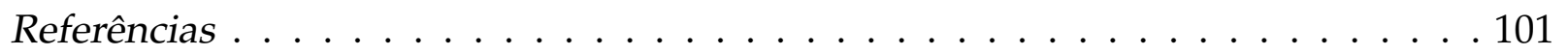

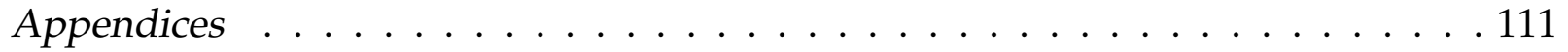




\section{Capítulo 1}

\section{Introdução}

O Mecanismo Flexível é um dispositivo mecânico utilizado para transformar movimento, força ou energia entre as portas de entrada e saída sem a presença de juntas ou pinos, baseando-se em uma estrutura monolítica, em outras palavras, a transformação do movimento é dada pela flexibilidade de sua estrutura. Deste modo, a transformação pode ser direcionada em um sentido específico, amplificando ou reduzindo o deslocamento ou força aplicados. Por este motivo são utilizados para concepção de atuadores piezelétricos flextensionais, onde as portas de entrada são acionadas por incertos de cerâmicas piezelétricas (ou "pilhas" de cerâmicas) que geram deslocamentos e/ ou forças em uma dada direção. Portanto têm aplicações em sensores e atuadores, eletromecânicos, piezelétricos para o controle de vibração estrutural, funcionamento de dispositivos com micro posicionamento e micro manipulação como: posicionadores de lentes em sistemas de interferometria laser, equipamentos para manipulação de células e sistemas micro eletromecânicos (Microelectromechanical Systems (MEMS)). São utilizados também em tecnologias mais recentes como a nanometrologia, equipamentos destinados à manipulação na nano escala (manipulação de nano tubos de carbono, por exemplo), sistemas nanoeletromecânicos (Nanoelectromechanical Systems (NEMS)) e igualmente aplicado a equipamentos de mecânica de precisão em geral (Ikeda, 1996; Anton e Sodano, 2007; Zhang et al., 2014; Hu et al., 2015; Uchino, 2010; Kögl e Bucalem, 2005; Silva, 2003).

Na figura 1.1 são apresentados dois tipos de atuadores piezelétricos flextensionais, 
o tipo "moonie"e o tipo "cymbal". Os atuadores do tipo moonie/cymbal foram desenvolvidos para amplificar os pequenos deslocamentos laterais induzidos pela cerâmica piezoelétrica, tendo características intermediárias dentre os atuadores bimórficos de múltiplas camadas convencionais.

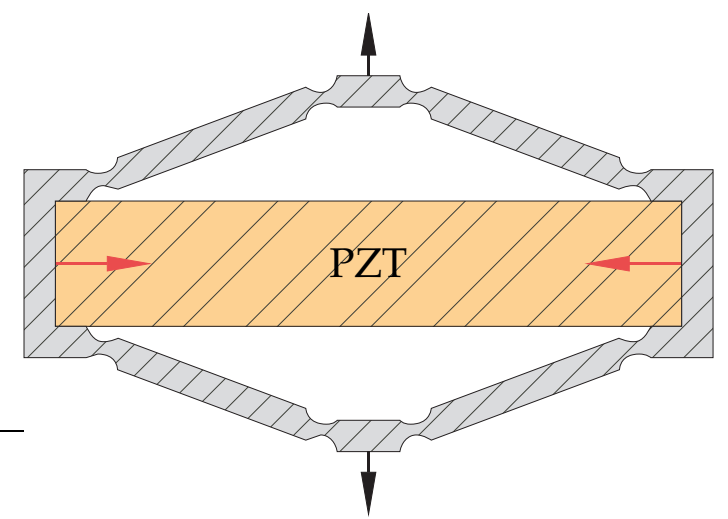

(a)

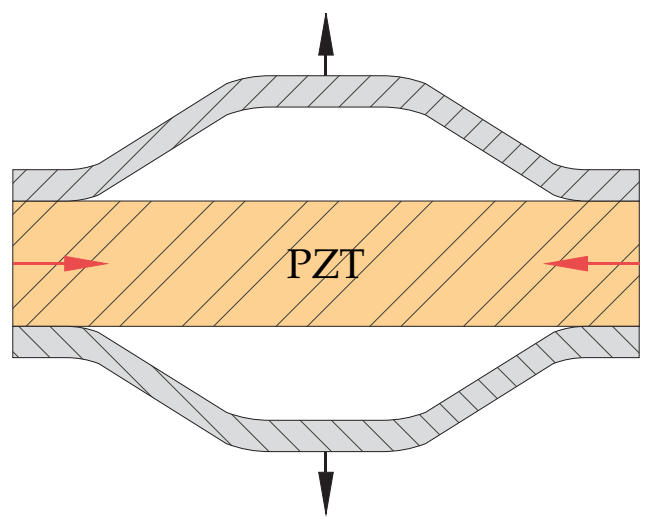

(b)

Figura 1.1: Exemplos de Atuadores Flextensionais piezelétricos, (a) atuador tipo "moonie", (b) "cymbal".

A maioria das classes dos atuadores flextensionais existentes geram deslocamentos da ordem de micrômetros, no entanto há uma classe de atuadores que geram grandes deslocamentos na ordem de milímetros ou mais e para isso utilizam-se do mecanismo de flambagem não-linear, ou seja utilizam a condição instável de sua estrutura para desta forma obter grandes deslocamentos na porta de saída. Neste tipo de atuador ou mecanismo o material estrutural utilizado em sua concepção é projetado para trabalhar em regime elástico, ou seja, sem efeito da plasticidade, pois se trata de um fenômeno irreversível, e tais mecanismos são projetados em regime de reversibilidade, para que possam operar múltiplas vezes.

Esta classe de atuadores que utilizam a flambagem não linear é apresentada na figura 1 (Iung e Yun, 2010), e são amplamente empregada na indústria de automação e aeroespacial pois possuem uma condição estrutural instável que é utilizada na maximização do deslocamento ou na coleta de energia. Um exemplo clássico deste tipo de atuador são os "twitters" utilizados em equipamento sonoros e de ultrassom. Outro exemplo de atuador que utiliza o mecanismo de flambagem não linear é o "THUNDER" (Mossi e 
Bishop, 1999), que é composto de materiais que incluem o PZT, adesivos poliméricos e uma camada metálica no substrato (aço inoxidável, alumínio, latão, cobre e etc.) prétensionada. Este tipo de transdutor tem uma montagem laminar conforme apresentado na figura 1.2 e o seu processo de fabricação envolve o aquecimento do material compósito à altas temperaturas $\left(325^{\circ} \mathrm{C}\right)$ seguido pelo resfriamento em temperatura ambiente para a inclusão do efeito de pré-tensão no substrato metálico. O laminado piezocompósito obtido neste processo é um dispositivo curvo cujo o deslocamento ou a força resultante na porta de saída é muito superior ao da porta de entrada e apresenta resultado melhor se comparado com uma cerâmica sozinha. Os atuadores de comportamento de flamba-

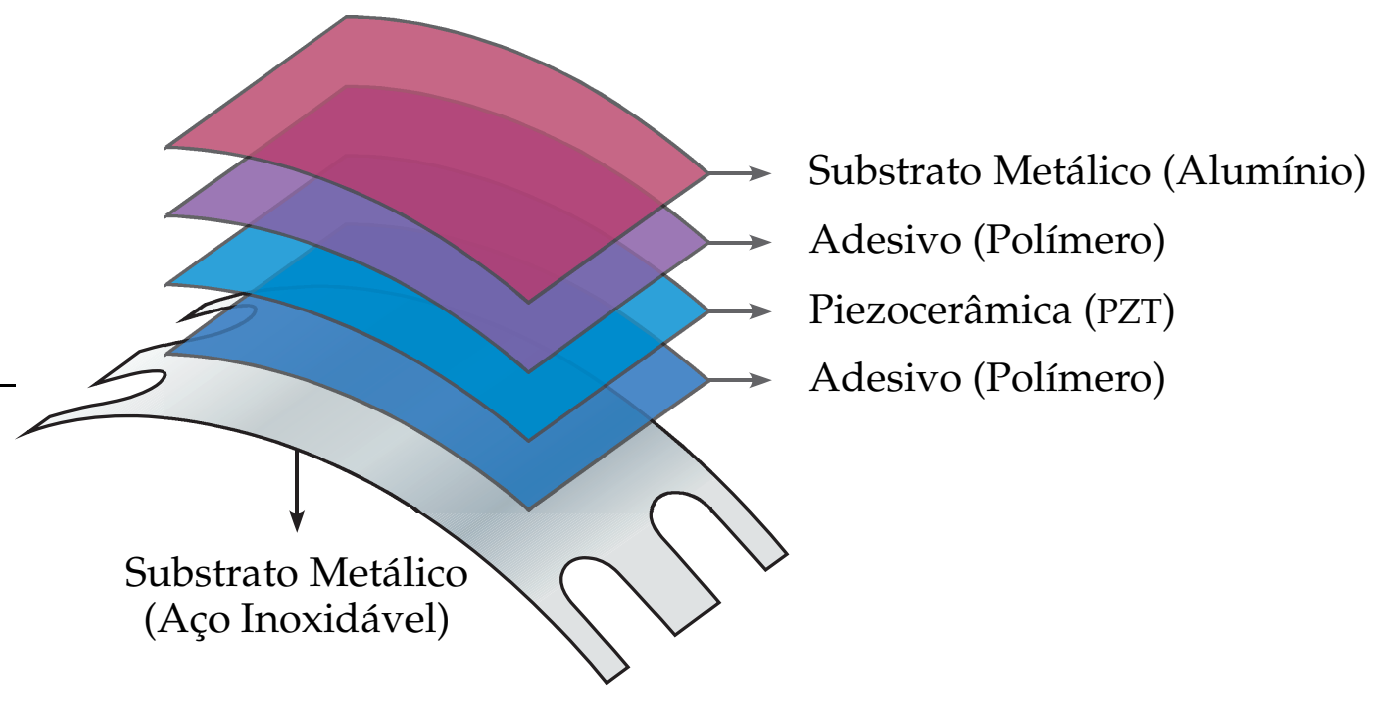

Figura 1.2: Constituição básica de um transdutor piezocompósito com múltiplas camadas.

gem não linear operam com deslocamento negativo com carga negativa e deslocamento positivo com carga positiva, conforme apresentado na figura 1 .

$\mathrm{Na}$ área de projetos de atuadores piezelétricos que utilizam a flambagem não linear, o trabalho de Schwartz e Naravanan (2002) disserta sobre o projeto de atuadores “THUNDER" alterando o efeito de pré-tensionamento do substrato metálico para um maior armazenamento de energia. Temos também o trabalho de Portela et al. (2008), onde numa placa de material compósito laminado de fibra de carbono pré-tensionada

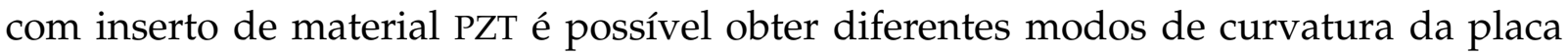
baseado no comportamento de flambagem não linear causada pela polarização do PZT. Em 2011 Tawfik et al. projetaram atuadores de comportamento de flambagem não linear utilizando placas laminadas pré-tensionadas em fibra de carbono empilhadas de forma 


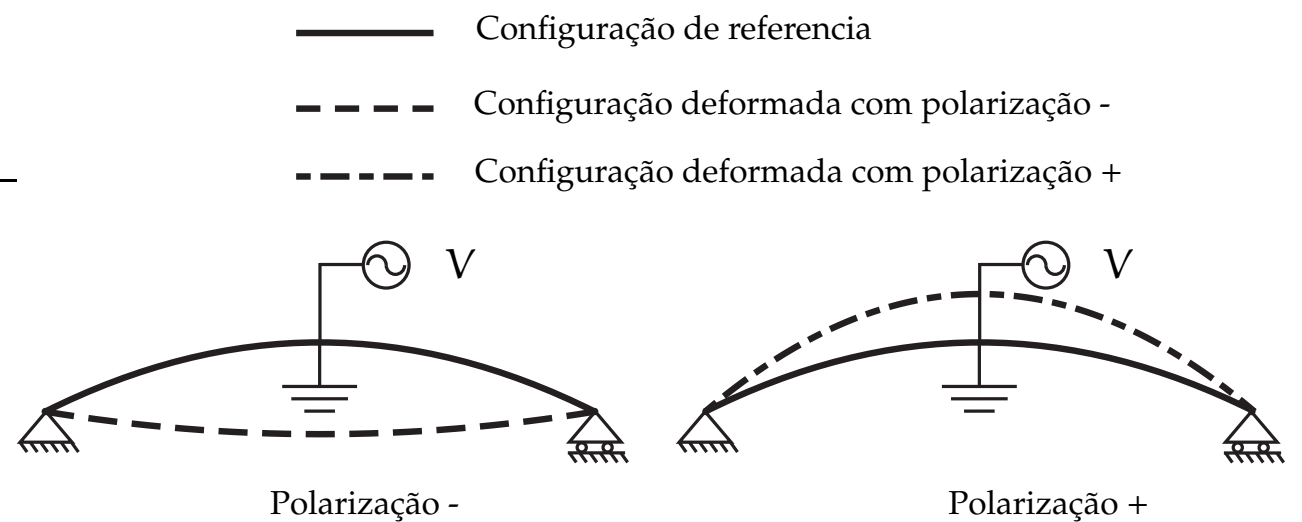

Figura 1.3: Configuração de trabalho do atuador piezelétrico que utiliza o mecanismo de flambagem não linear

não simétrica, demonstrando desta forma sua eficiência e viabilidade de construção, quando comparados com resultados experimentais.

Métodos e algoritmos de otimização podem ser utilizados para projetar de modo mais eficiente tais mecanismos e atuadores flextensionais. Um dos métodos de otimização mais robusto é o Método de Otimização Topológica (MOT), que foi utilizado neste projeto para projetar esta classe de mecanismos flexíveis. Este trabalho investigou diversas técnicas de otimização topológica, visando obter a topologia da estrutura que respeitasse um modo de flambagem não linear pré determinado, considerando os deslocamentos e forças nas portas de entrada e saída do mecanismo.

\subsection{Projeto de Mecanismos Flexíveis considerando o efeito da flambagem não-linear via MOT}

$\mathrm{OMOT}$ consiste num método computacional que distribui o material no interior de um domínio fixo de projeto de forma a minimizar uma função custo especificada (por exemplo, máxima rigidez com uma restrição de volume de material). Ao contrário dos métodos de otimização paramétrica e de forma que não permitem grande liberdade no projeto (Vanderplaats, 1984; Haftka e Gürdal, 2012), o MOT permite determinar onde é possível gerar novos furos na topologia da estrutura, mantendo a máxima eficiência na 
relação massa/rigidez, por exemplo, aumentando desta forma o desempenho da estrutura (Bendsøe e Kikuchi, 1988) e permitindo grande liberdade para desenvolver novos conceitos (ver figura 1.4). Este método é largamente utilizado nas indústrias automobilística e aeronáutica, nos Estados Unidos, Japão e Europa para o projeto de componentes mecânicos, além de recentemente ter se expandido para outras áreas da Engenharia no meio acadêmico, como o projeto de mecanismos flexíveis, micro-mecanismos flexíveis com atuação térmica (Jonsmann, 2000), atuadores flextensionais, MEMS, NEMS, antenas e motores eletromagnéticos, etc (Kögl e Silva, 2005; Silva et al., 2000; Bendsoe e Sigmund, 2013). O processo de OT é essencialmente um método numérico, pois, se baseia na

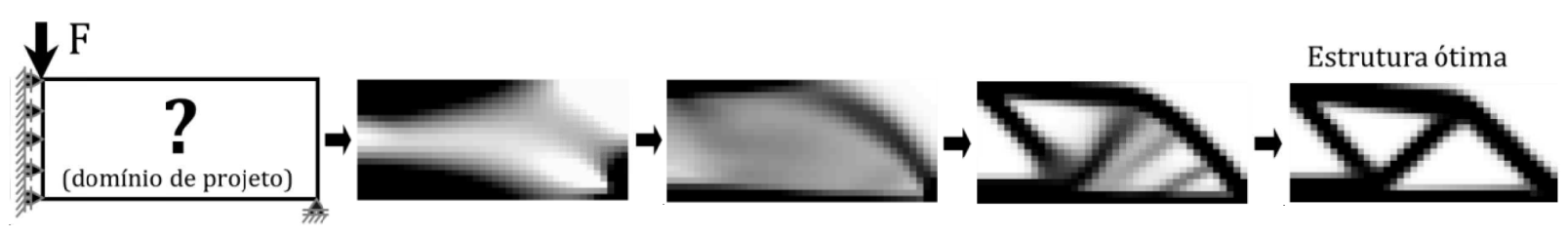

Figura 1.4: Exemplo de aplicação do MOT

maioria dos casos no MEF e num algoritmo de otimização numérico para encontrar a ótima distribuição de material no interior de um domínio inicial discretizado em elementos finitos, que permanecerá fixo durante o processo iterativo (Bendsøe, 1989). A cada iteração, a distribuição de material é alterada e o domínio corrente é analisado pelo MEF para o cálculo da função objetivo e restrições de projeto. Depois de satisfeito o critério de convergência, obtém-se como resultado a distribuição ótima de material ao longo do domínio de projeto.

Mecanismos flexíveis são uma classe de dispositivos mecânicos em que a deformação elástica é utilizada como meio de se obter uma transformação do movimento entre as portas de entrada e saída. Na concepção de mecanismos convencionais, onde a ligação das juntas de movimento são rígidas, a deformação elástica é geralmente indesejável. Porém, os mecanismos flexíveis são projetados para serem intencionalmente flexíveis, para que sua construção seja em uma única peça ou monolítica. Sua construção tem algumas vantagens em relação aos mecanismos convencionais, como, por exemplo, não necessitam de lubrificação em suas juntas, não tem atrito, não sofrem desgaste e não possuem folgas, o que torna sua aplicação ideal para a indústria de alta precisão. 


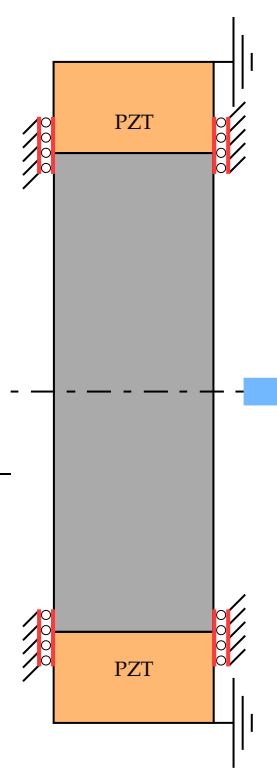

(a)

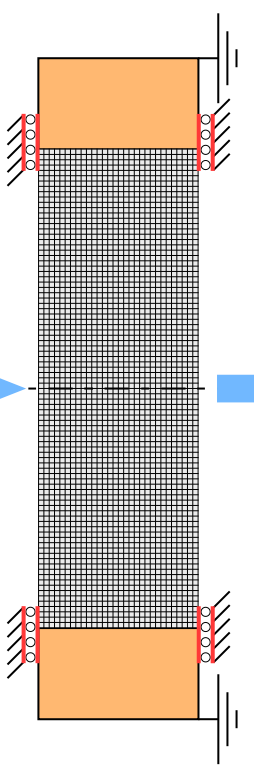

(b)

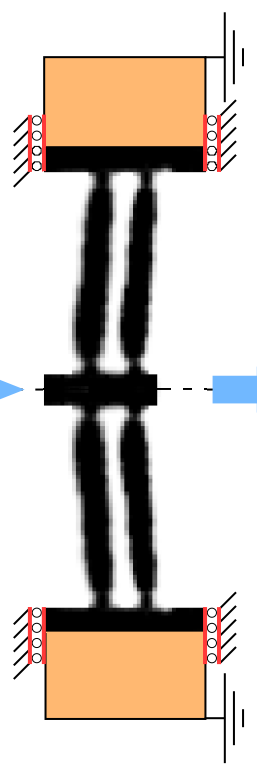

(c)

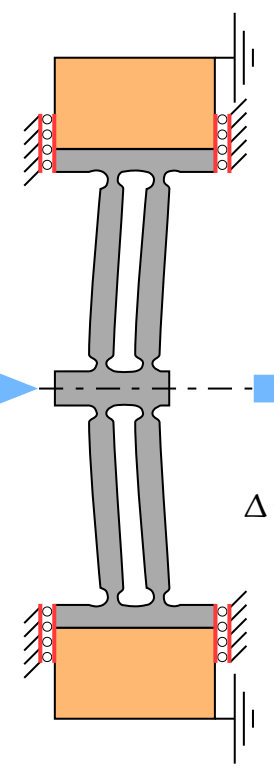

(d)

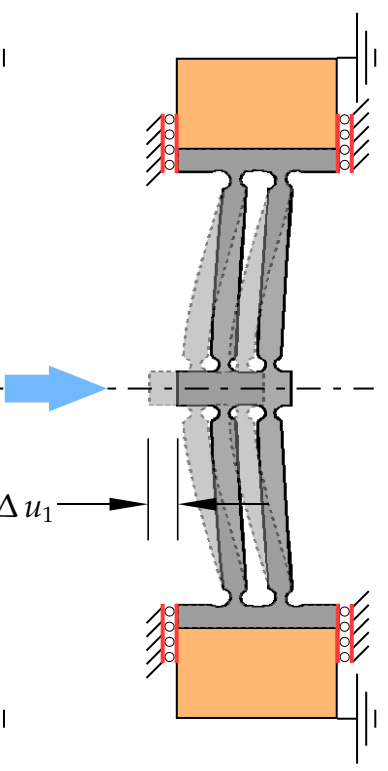

(e)

Figura 1.5: Procedimento de projeto de atuadores flextensionais que utilizam o efeito da flambagem não linear via o MOT.

(a) Domínio de Projeto. (b) Domínio de Projeto Discretizado.

(c) Resultados do processo de OT (d) Interpretação dos resultados (CAD).

(e) Verificação dos resultados (MEF)

Em geral a sua concepção é baseada nos coeficientes de eficiência mecânica e/ou geométrica (em inglês denominado por Mechanical Advantage (MA) e Geometrical Advantage (GA)) que relacionam a transformação entre as portas de entrada e saída (ver Seção 4.6). Os primeiros trabalhos na área utilizando o MOT inicialmente desenvolvido por Ananthasuresh (1994) utilizaram técnicas de otimização e o Método de Homogeneizacão (MH). Os trabalhos de Larsen et al. (1997); Sigmund (1997); Kikuchi e Kota (1997); Nishiwaki et al. (1998) propuseram o uso do MOT como o modelo de material SIMP para a concepção deste dispositivo mecânico, emergindo basicamente duas formulações. A primeira baseada na maximização dos deslocamentos na porta de saída, onde uma mola é fixada para impor uma rigidez artificial e auxiliar na formação de uma estrutura na porta de saída. A segunda formulação consiste em dividir o problema elástico em dois casos de carregamentos distintos, um carregamento na porta de entra e outro na porta de saída, combinando os dois carregamentos via deslocamento em uma função denominada de energia mútua Nishiwaki et al. (1998). Ambas as formulações demonstraram a grande potencialidade do MOT para projetar esta classe de dispositivos, no entanto, as metologias desenvolvidas não levavam em consideração as grandes 
deformações, deslocamentos e rotações.

Com relação à aplicação do MOTutilizando a mesma metodologia aplicada a mecanismos flexíveis, este conceito foi estendido por Silva et al. (2000) para o desenvolvimento de atuadores flextensionais através da expansão dos conceitos de energia mútua e flexibilidade média adicionada ao MOT. Assim foi possível projetar mecanismos flexíveis e atuadores piezelétricos especificando a funções objetivo e restrições de projeto, viabilizando de forma genérica e sistemática projetos não convencionais e inovadores como garras, grampos, pinças e etc (Carbonari et al., 2007).

Posteriormente os trabalhos de Pedersen et al. (2001); Bruns e Tortorelli (2001) apresentaram metodologias muito semelhantes que levavam em conta os grandes deslocamentos da estrutura, ou seja, o problema de elasticidade era não linear, ou seja, a sensibilidade considerava esta não linearidade. No entanto para estabilizar os elementos de baixa densidade foi utilizada a lógica proposta por Buhl et al. (2000), onde a força interna dos elementos de baixa densidade eram retirados do cálculo do equilíbrio.

O trabalho de Bruns et al. (2002b) foi o primeiro a incluir a flambagem não linear como uma restrição de projeto para sintetizar a topologia de mecanismos flexíveis via MOT, desenvolvendo uma metodologia de restrição do caminho do incremento de carga utilizando o Método das Assíntotas Móveis (ㅍM), porém, foi necessário melhorar o Método do Comprimento de Arco (MCA) devido a problemas de convergência advindo dos elementos de baixa densidade. No entanto a instabilidade causada pelos elementos de baixa densidade só seria realmente explicada no trabalho posterior de Lahuerta et al. (2013), onde testes numéricos demonstraram a importância da utilização de modelos constitutivos policonvexos (ver Seção 2.4).

Entretanto, o primeiro trabalho de Bruns et al. (2002b) levantou mais questões do que respostas sobre a metodologia apresentada. Devido a este motivo a continuação do trabalho (Bruns e Sigmund, 2004) detalhou melhor a metodologia desenvolvida e mais resultados foram apresentados. Sendo esta nova metodologia dividida em três fases, sendo cada uma responsável por projetar um ponto do caminho do carregamento 
instável. Quando este ponto era obtido, o valor de carga era incrementado, aumentando o número de incrementos de controle e a formulação do problema era modificado, com a finalidade de se manter os pontos instáveis anteriores e buscar por um novo.

Ohsaki e Nishiwaki (2005) também propuseram uma outra metodologia de projetar tais dispositivos impondo as restrições de deslocamento, incremento de carga e massa em uma única fase de concepção utilizando o algoritmo de otimização de Programação Quadrática Sequencial ( $\overline{\mathrm{PQS}}$, porém, utilizando um fator de relaxação das restrições para facilitar a convergência do problema. Conforme as restrições de projeto são respeitadas este fator de relaxação é minimizado até ser completamente removido do problema de otimização.

Examinando em detalhes às três metodologias apresentadas por ambos os autores Bruns et al. (2002a); Bruns e Sigmund (2004); Ohsaki e Nishiwaki (2005) fica explicito que o problema principal está relacionado ao valor inicial das variáveis duais (ver Seção 3.7) e não à violação das restrições. O trabalho de Bruns e Sigmund (2004) propõem justamente melhorar o valor inicial de cada fase, possibilitando a solução pelo MAM. Ajustes similares nas restrições de projeto foram feitas por Ohsaki e Nishiwaki (2005), porém, de uma maneira mais eficaz. O que leva a uma pergunta importante sobre o como projetar estruturas sujeitas a flambagem não linear: A formulação do problema de otimização é consistente? A utilização do MOT com MAMe o[QS baseado na formulação lagrangiana é adequada a este tipo de problema? São questões importantes que necessitam serem respondidas dadas as dificuldades do tema.

A figura 1.5 apresenta um esquema que resume os passos a serem seguidos num procedimento de projeto estrutural utilizando OT aplicado a mecanismos flexíveis biestáveis, baseado no efeito da flambagem não linear, onde a flambagem é desencadeada pela polarização do PZT que geram deslocamentos nas respectivas portas de entrada. 


\subsection{Revisão Bibliográfica}

A revisão bibliográfica do projeto de mecanismos flexíveis e atuadores flextensionais é um pouco extensa, pois, se trata de um tema que engloba diversas áreas do conhecimento, no entanto, se separamos somente uma revisão relativa a flambagem não linear o número de trabalhos se reduz muito. Portanto, um resumo cronológico é necessário para compreender a evolução, desafios e estágio atual do seu desenvolvimento em relação à parte teórica, de equacionamento via EDPs utilizando o MEFe o MOT, Faremos uma distinção entre os tipos de flambagem, para flambagem linear a referência se faz em relação a uma análise linear, com o objetivo de predizer os modos de flambagem e de carga crítica através do uso dos autos-valores e vetores da matriz rigidez elástica e geométrica (para mais detalhes ver Capítulo 7.1.1 do livro (Wriggers, 2008)).

A flambagem é um fenômeno de instabilidade estrutural, onde a estrutura entra em colapso por motivos de cinemática ou ligados a efeitos locais de material que alteram a cinemática estrutural acarretando instabilidade. O mecanismo de flambagem pode ser analisado de forma aproximada, ou seja somente consideram os efeitos de pré-tensão e geométricos (imperfeições) em uma análise de auto-valor e vetor com o propósito de determinar os valores de carga limite e modos de flambagem. Entretanto, a flambagem pode ser analisada considerando todo o equilíbrio não linear da estrutura, onde a cinemática da estrutura é tratada de forma mais precisa e, portanto, é possível determinar a configuração atualizada, obtendo desta maneira o valor de incremento de carga em cada ponto de instabilidade, também referida na literatura como análise de pós flambagem, pois, após a primeira flambagem não linear da estrutura outras configurações instáveis são analisadas até uma condição limite (para mais detalhes ver Capítulo 7.1.2 do livro (Wriggers, 2008)).

Inicialmente o projeto de mecanismos e atuadores não se embasava em ferramentas de base física analítica onde uma equação ou um conjunto de equações eram responsáveis pela sua concepção (Crawley e De Luis, 1987). A maioria dos projetos destes atuadores eram feitas com o conhecimento experimental adquirido ao longo diversas 
tentativas e erros de projeto. Esta revisão bibliográfica é direcionada somente à revisão dos mecanismos flexíveis e atuadores flextensionais que trabalham de alguma forma com o mecanismo de flambagem, por esta ser a motivação deste trabalho.

A ideia inicial de projetar mecanismos e atuadores como uma estrutura inteligente ou com alguma característica física que pudesse ser alterada a partir de uma perturbação externa foi inicialmente proposta por Crawley e De Luis (1987), como já mencionado anteriormente estes atuadores são basicamente estruturas flexíveis com portas de entrada e saída que transferem ou transformam o movimento, força ou energia gerada pela piezocerâmica, também denominada de mecanismos flexíveis. A combinação entre mecanismos flexíveis e material[PZT|constituem um atuador piezoelétrico.

Alguns anos após Crawlev e De Luis (1987) terem proposto o projeto de estruturas inteligentes, Meressi e Paden (1993) verificaram a possibilidade de controlar os primeiros modos de flambagem de uma viga prismática biapoiada, aplicando duas camadas externas de material PZT. Quando o material Titanato Zircanato de Chumbo (PZT) era polarizado notou-se alterações nos valores de carga limite e dos modos de colapso da viga, ou seja era o início do controle de certas características físicas ou mecanismos de uma estrutura inteligente. Em seguida, Sigmund (1997) apresentou uma nova metodologia para o projeto de mecanismos flexíveis lineares, utilizando o MOT, uma ferramenta mais sistemática e muito poderosa que não se limitava a elementos de viga e ao projeto ótimo de estruturas rígidas. Era uma metodologia que permitia trabalhar com qualquer outro tipo de elemento ou discretização numérica capaz de conceber a forma e topologia de mecanismos flexíveis.

Baseado na potencialidade do MOT, Silva e Kikuchi (1999) propuseram a inclusão do efeito eletromecânico dos materiais PZT desenvolvendo uma metodologia capaz de projetar transdutores PZT de alto desempenho, aproximando linearmente as deformações elásticas no cálculo do equilíbrio e gradientes de forma iterativa. Foi utilizado o algoritmo otimização numérico Programação Linear Sequencial (록 $)$ com informações dos gradientes das funções objetivo e de restrições, obtendo de forma iterativa a distribuição ótima de material de modo a criar uma estrutura ou mecanismo capaz de amplificar 
ou reduzir os deslocamentos na porta de saída quando os insertos de material $\overline{\text { PZT }}$ estivessem polarizados nas portas de entrada. No entanto, o MOT era utilizado somente com uma aproximação linear do variacional relativo ao equilíbrio eletromecânico, desta forma os deslocamentos, rotacões e deformacões eram aproximados linearmente (Bendsoe e Sigmund, 2013). Portanto, havia a necessidade de um grande número de variáveis de projeto para que fosse possível obter uma distribuição de material que gerassem resultados interpretáveis, requisitando desta maneira um grande esforço computacional para a obtenção de resultados relevantes em relação ao processo de manufatura. Se considerarmos a solução completa do equilíbrio não linear da estrutura, o custo computacional é muitas vezes superior em comparação a aproximação linear, relato feito no trabalho de Pedersen et al. (2001). Por este motivo que poucos trabalhos que considerassem a solução do equilíbrio não linear no MOTforam publicados até aquele momento (Iog, 1996; Buhl et al., 2000; Gea e Luo, 2001).

Trabalhos com o enfoque teórico e de aplicação do MOT considerando a não linearidade elástica ou geométrica foram apresentados por Buhl et al. (2000); Bruns e Tortorelli (2001); Bruns et al. (2002b); Gea e Luo (2001); Pedersen et al. (2001), que dedicaram estudos sobre este tema e fizeram uso de muitos artifícios numéricos sem consistência física para estabilizar o MOT. Somente depois de alguns anos foi proposta uma formulação mais consistente (Lahuerta et al., 2013; Penzler et al., 2012; Wallin e Ristinmaa, 2015) que utilizava uma lei material policonvexa, mas estes trabalhos se limitaram na utilização do método somente na pré flambagem, ou seja, o mecanismo de instabilidade estrutural era negligenciado. Os trabalhos de Bruns et al. (2002a); Bruns e Sigmund (2004); Ohsaki e Nishiwaki (2005) propuseram depurar o assunto sobre flambagem não linear, pois, ainda não existia uma formulação geral do MOT aplicada para a síntese de tais estruturas que considerassem tal mecanismo de colapso. O trabalho de Bruns et al. (2002a) propôs projetar um mecanismo flexível que tivesse um comportamento não linear entre os carregamentos na porta de entrada e saída, ajustando uma curva de pós-flambagem. Tal comportamento poderia ser ajustado ao longo do processo de otimização impondo uma função que minimizasse o erro entre o comportamento real e o desejável da estrutura flexível, com restrições de carregamento, utilizando o Método das Assíntotas Móveis (MAM), para obter a ótima distribuição de material. No entanto, tais resulta- 
dos apresentavam uma grande escala de cinza, eram difíceis de serem reproduzidos e muito dependentes do valor inicial das variáveis de projeto, resultando em um trabalho posterior mais aprofundado (Bruns e Sigmund, 2004).

A continuação da pesquisa de Bruns e Sigmund (2004) forneceu uma metodologia mais geral para a síntese deste tipo de mecanismos que trabalham na pós flambagem. Esta metodologia foi dividida em três fases, sendo cada uma responsável por projetar um ponto de instabilidade na pós flambagem. Quando um ponto instável era obtido, o valor de carga era incrementado, aumentando o número de incrementos de controle e a formulação do problema era modificado, com a finalidade de se manter o ponto instável já obtido e buscar um novo. Comparando os trabalhos Bruns e Sigmund (2004) e de Ohsaki e Nishiwaki (2005) verificamos que o último também propôs a utilização de restrições de deslocamento e de incremento de carga, entretanto, as restrições foram aplicadas em uma única fase de projeto de forma direta no algoritmo de otimização de $\mathrm{PQS}$, utilizando um fator de relaxação das restrições para facilitar a convergência do problema. Conforme as restrições de projeto são respeitadas este fator de relaxação é minimizado até ser completamente removido do problema de otimização.

Examinando em detalhes às três metodologias apresentadas por ambos os autores Bruns et al. (2002a); Bruns e Sigmund (2004); Ohsaki e Nishiwaki (2005) fica explicito que o problema principal está relacionado ao valor inicial e não à violação das restrições impostas. O trabalho de Bruns e Sigmund (2004) propõem justamente melhorar o valor inicial de cada fase, possibilitando a solução pelo MAM Ajustes similares nas restrições de projeto foram feitas por Ohsaki e Nishiwaki (2005), porém, de uma maneira mais eficaz. O que leva a uma pergunta fundamental sobre o projeto de estruturas na pósflambagem: A utilização do MOT, OMAM o $\mathrm{PQS}$ é adequada para este tipo de problema? Ou são aplicados de forma inadequada? Esta é uma questão levantada devido ao uso de diversos artifícios numéricos apresentados na literatura e necessários para que as restrições de deslocamento e carregamento não sejam violadas pelos respectivos métodos supramencionados.

Prosseguindo com a cronologia, em 2006 o trabalho de Cardoso e Fonseca (2006) 
propôs o projeto de atuadores piezoelétrico considerando a não linearidade geométrica, onde resultados muito similares entre a aproximação linear e não linear eram apresentados. Todavia o trabalho de Pedersen et al. (2001) já havia apresentado resultados divergentes de mecanismos com topologias diferentes considerando linearidade e não linearidade geométrica. Esta abordagem foi capaz de avaliar o comportamento físico de forma numérica da classe de atuadores "Thunder" (Capozzoli et al., 1999) utilizando o MEF, pois incluindo um carregamento térmico foi possível adicionar o efeito da pretensão em uma casca laminada e avaliar o seu comportamento quando o material PZT era polarizado. Uma aproximação não linear geométrica para o projeto de "filmes atuadores" ou atuadores laminares muito finos e flexíveis utilizando o MOT foi sugerida também por Howard et al. (2009), considerando o efeito da flambagem não linear com a finalidade de projetar curvaturas quando o filme de material[PZT estivesse polarizado.

Somente em 2013 o trabalho de (Lindgaard e Dahl, 2013) buscou uma formulação geral mais consistente de como projetar estruturas considerando a flambagem não linear via MOT, Definições e derivadas de funções que permitem predizer pontos de instabilidade foram incluídas como funções de restrições no processo de OT, porém a formulação não linear relativa a não linearidade geométrica era muito simplista, com muitos truques na penalização no modelo material, mas se compararmos com o trabalho de Bruns e Sigmund (2004), foi uma grande evolução. Entretanto é um metodologia que busca maximizar a rigidez e que não considera o projeto de mecanismo flexível ou atuador flextensional.

O trabalho de Hu et al. (2015) se dedica a demonstrar a importância da flambagem de forma geral, sem mencionar qualquer formulação para o projeto de atuadores que utilizem o mecanismo de pós-flambagem via MOT, demonstrando ser um tema de grande relevância nas áreas acadêmica e industrial na atualidade. $\mathrm{O}$ artigo de James e Waisman (2016) descreve uma solução geral aparentemente simples para sintetizar atuadores biestáveis que utilizam o mecanismo de flambagem não linear. A formulação apresentada é muito semelhante com a terceira fase de projeto proposta pelo trabalho de Bruns e Sigmund (2004), porém, aplicada de forma direta no MAMe com o objetivo de projetar Stents cardíacos. No entanto, sua efetividade é discutível já que os autores 
Bruns e Sigmund (2004); Ohsaki e Nishiwaki (2005) foram enfáticos na dificuldade de se impor restrições de flambagem não linear de forma direta utilizando restrições de força de reação.

\subsection{Objetivos}

O objetivo deste trabalho é desenvolver uma metodologia de projeto de mecanismos flexíveis baseados no efeito da flambagem não linear utilizando o MOT, Será explorada a potencialidade da não linearidade elástica e geométrica nesta classe de mecanismo buscando os seguintes objetivos:

- Melhoria da formulação de MEF aplicada à OT considerando a não linearidade geométrica com a finalidade de estabilizar as regiões com elementos de baixa densidade;

- Formulação e implementação do MEFbaseada em elementos contínuos para simulação de domínios que levem em conta as propriedades elásticas, utilizando um modelo constitutivo policonvexo;

- Desenvolver uma formulação no MOT para restrições de projeto que envolvam flambagem não-linear, determinando a ótima distribuição de material;

\subsection{Justificativa}

Mecanismos flexíveis baseados no efeito da flambagem não linear utilizados atualmente são projetados com pouco uso de ferramentas computacionais, empregando apenas ferramentas de validação de conceito. Isto gera um alto custo de desenvolvimento, pois, necessita diversas reavaliações e adaptações para obter de um resultado simples e funcional, sem explorar o conceito da flambagem em toda a sua potencialidade. Baseado nesta premissa, esta tese ter por objetivo desenvolver uma metodologia 
eficiente utilizando métodos computacionais, como o MOT para o projeto e desenvolvimento de mecanismos que operem sob flambagem não linear.

A grande vantagem da utilização do mecanismo de flambagem não-linear nesta classe de dispositivo mecânico é o efeito amplificador dos deslocamentos gerados pela condição estrutural instável, resultando em mecanismos de alto desempenho.

A formulação de OT desenvolvida neste trabalho permitirá o projeto de mecanismos flexíveis inovadores que poderão ser utilizados para desenvolver atuadores flextensionais piezelétricos inovadores, gerando enormes ganhos de eficiência e aplicação.

\subsection{Contribuições Científicas}

Até o momento (2017) a literatura sobre o projeto de mecanismos flexíveis considerando o efeito da flambagem não linear é pequena (Bruns et al., 2002a; Bruns e Sigmund, 2004; Ohsaki e Nishiwaki, 2005; Iames e Waisman, 2016) e, no entanto se utiliza de muitos artifícios numéricos e de restrições de projeto. Portanto o desenvolvimento de uma metodologia mais consistente e robusta com um forte embasamento teórico terá grande relevância na área.

A metodologia desenvolvida nesta tese propõe soluções para grande parte das dificuldades apresentadas pelos autores citados (Lindgaard e Dahl, 2013; Bruns e Sigmund, 2004; Ohsaki e Nishiwaki, 2005; Iames e Waisman, 2016) e foi dividida em quatro tópicos importantes:

- Restrições não lineares. Como relatado pelos trabalhos de (Lindgaard e Dahl, 2013; Bruns e Sigmund, 2004; Ohsaki e Nishiwaki, 2005) as restrições de deslocamento e força interna na pós-flambagem são muito difíceis de serem respeitadas pelo algoritmo de otimização nas iterações iniciais. Geralmente tais restrições encontram-se fora do domínio viável de projeto e muitos algoritmos apresentam 
sérias dificuldades em encontrar uma topologia inicial que não as violem, mesmo utilizando uma funcão Beta dentro da funcão objetivo (Haftka e Gürdal, 2012; Vanderplaats, 1984). Analisando estas dificuldades previamente relatadas, decidiu-se por utilizar o Método dos Mínimos Quadrados (MMQ) em conjunto com o Método do Ponto Interior (MPI) para facilitar a estimativa das variáveis duais do problema de otimização, ou seja, as restrições de projeto relativas a flambagem não linear são impostas de forma gradativa, evitando assim instabilidades na convergência do processo de otimização, o que consiste em contribuição.

- Formulação do problema de OT. Foi proposta uma função multiobjetivo baseada na somatória de múltiplos casos de carga para obtenção de mecanismos flexíveis considerando a elasticidade não linear, onde o primeiro caso de carregamento é referente ao carregamento "real"do mecanismo. Os demais casos de carga atribuise um carregamento unitário em cada uma das portas de entrada e saída, somente para impor rigidez na estrutura das portas de saída, atribuída pela minimização da somatória da energia de deformação do seu respectivo caso de carga.

- Derivada do Incremento de Carga. Foi implementada a sensibilidade do incremento de carga consistente com relação aos MCA e Método do Controle Generalizado dos Deslocamentos (MCGD) para aplicação direta no MOT, possibilitando desta forma impor restrições de projeto relativa ao incremento de carga aplicado na estrutura, sem a necessidade de incluir molas na região de aplicação da carga.

- Método de solução não linear. Foi selecionado o MCGD como um recurso para o cálculo da trajetória de equilíbrio na pós flambagem devido a diversas vantagens que o método possui em relação aos demais MCA, como cálculo automático do incremento de arco e o cálculo do Parâmetro Generalizado de Rigidez (PGR) (Yang e Shieh, 1990; Cardoso e Fonseca, 2007). Somente o cálculo da trajetória de equilíbrio não é suficiente para obter o seu incremento ou de pseudo tempo de modo preciso como parâmetro, necessário para o impor as restrições de projeto no MOT. Lembrando que, o incremento de arco é uma projeção do incremento de carga em relação ao vetor dos deslocamentos e, portanto, é muito difícil antever o incremento de arco necessário para obter o incremento de carga desejado na trajetória de equilíbrio na pós-flambagem do mecanismo. Para solucionar esta 
questão, foi implementado um algoritmo de interpolação dos vetores de deslocamento utilizando uma função de base radial euclidiana (Franke, 1982; Carr et al., 2001; Rippa, 1999), que minimiza a norma do erro da função interpoladora em relação aos dados de entrada. Sendo este um método empregado também para a reconstrução de imagens, porém, não foi encontrado até o presente momento nenhuma referência ou trabalho que utilize este algoritmo de interpolação no MOT com a finalidade de obter o vetor das variáveis primais na configuração atualizada do modelo em um ponto do incremento de carregamento desejado em todo o cálculo da flambagem não linear.

\subsection{Organização da Tese}

No Capítulo—2 é feita uma descrição teórica sobre a mecânica do contínuo, tensores de deformação, leis materiais, formulação da forma fraca e o acoplamento mecânico. A implementação numérica relativa a linearização, MEF, métodos de solução de sistemas, métodos numéricos de otimização, MOTe sensibilidade são descritos no Capítulo 3. No Capítulo 4é feito um descritivo de aspecto teórico do MOT, modelo de material, função de continuação, problemas associados ao MOT, técnica de projeção, análise de sensibilidade. Os resultados com a metodologia proposta são apresentados no Capítulo 5, onde são feitos diversos testes com a formulação proposta considerando a flambagem não linear. Por fim, no Capítulo 6 são apresentadas as conclusões da tese e trabalhos futuros. No apêndice 6.1 são apresentadas formulações relativas ao CO, MAM que geralmente são utilizadas no MOT, 


\subsection{Notação Matemática}

A notação matemática utilizada neste trabalho segue o seguinte padrão:

- Letras minúsculas latinas ou gregas $(a, b, \ldots \alpha, \beta \ldots)$ representam grandezas escalares;

- Letras minúsculas latinas ou gregas em negrito-itálico $(\boldsymbol{a}, \boldsymbol{b}, \ldots \boldsymbol{\alpha}, \boldsymbol{\beta} \ldots)$ denotam vetores;

- Letras maiúsculas latinas ou gregas em negrito-itálico $(\boldsymbol{A}, \boldsymbol{B}, \ldots)$ expressam tensores de segunda ordem;

- Letras maiúsculas latinas ou gregas em negrito-itálico utilizando a fonte "Euclid Math One" ( $\mathcal{A}, \mathcal{B}, \ldots)$ expressam tensores de terceira ordem;

- Letras maiúsculas latinas ou gregas utilizando a fonte "Euclid Math Two" ( $\mathbb{A}, \mathbb{B}, \ldots)$ expressam tensores de quarta ordem;

- Matrizes e vetores construídos com componentes tensoriais são expressos por letras latinas em negrito $(\mathbf{A}, \mathbf{B}, \ldots \mathbf{a}, \mathbf{b} \ldots)$;

Lembrando que todas as grandezas estão no espaço Euclidiano tridimensional.

O espaço vetorial linear é descrito por $\mathcal{V}_{n}$, no qual $n$ é referente a dimensão do espaço. Adota-se ainda a convenção de somatório de Einstein sobre índices repetidos, estando subentendido que estes assumem valores entre $\{1,2\}$ para letras gregas ou entre $\{1,2,3\}$ para letras latinas, ambas com representação minúscula. Para descrever derivadas de uma função, tensor ou matriz utiliza-se esta convenção, onde a derivada é representada por vírgula com sua respectiva direção indicial. 


\section{Capítulo 2}

\section{Formulação Teórica}

Neste Capítulo são apresentados conceitos importantes para a formulação teórica da cinemática aplicada ao projeto de mecanismos flexíveis.

\subsection{Mecânica do Contínuo}

Neste item é abordado um resumo sobre a mecânica do contínuo, como relações cinemáticas, as leis de equilíbrio com as equações constitutivas que são necessárias para a solução do problema via MEF (Pimenta, 2008).

As relações cinemáticas são definidas com base no sistema de coordenadas inerciais na configuração de referência $\boldsymbol{e}_{\mathfrak{i}}^{r}$. Baseado nesta premissa as medidas de deformações são introduzidas. Portanto a formulação variacional pode ser definida para posterior uso no MEF,

A posição de um ponto material qualquer na configuração deformada é definida por:

$$
x=x^{\mathrm{r}}+u
$$

no qual os vetores $x^{\mathrm{r}}$ e $x$ são referentes a configuração de um ponto material na posição 


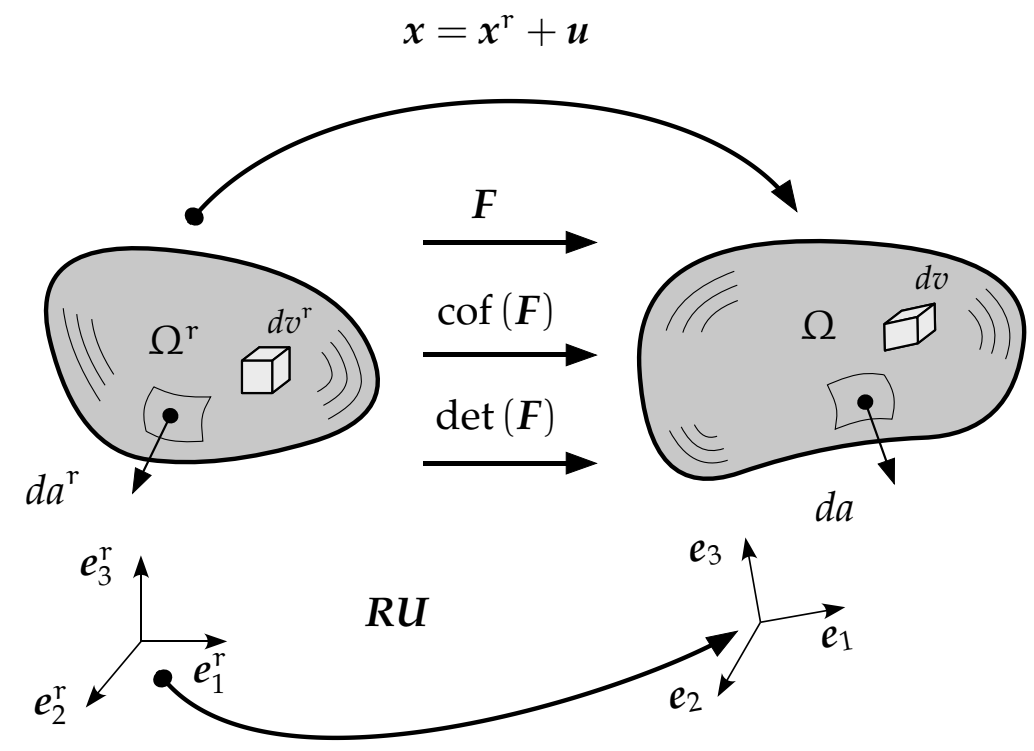

Figura 2.1: Configurações de um sólido no espaço.

de referência $(\bullet)^{\mathrm{r}}$ e atualizada $(\bullet)$ e o vetor de deslocamento é dado por $\boldsymbol{u}$ como ilustrado pela figura 2.1. A função que faz a transformação linear entre ambas as configurações é o gradiente da transformação e é dada por

$$
\boldsymbol{F}=\frac{\partial x}{\partial x^{r}}=\frac{\partial x}{\partial \eta_{i}} \otimes \boldsymbol{e}_{i}^{r}=f_{i} \otimes \boldsymbol{e}_{i}^{r}=I+\frac{\partial u}{\partial \eta_{i}} \otimes \boldsymbol{e}_{i}^{r} \quad \in \mathbb{R}^{n \times n}
$$

onde $\boldsymbol{F}$ pode ser descrito pelas suas componentes do vetor de base na configuração de referência $\frac{\partial x}{\partial \eta_{i}}=f_{i}$ ou pelas componentes do gradiente dos deslocamentos $\gamma_{i}=\frac{\partial u}{\partial \eta_{i}}$ que serão utilizadas ao longo do texto para facilitar as deduções das equações de movimento e equilíbrio (Pimenta, 2008). Portanto, é um tensor de campo de dois pontos (two-point) ou um vetor duplo, pois, é composto por um produto vetorial de dois vetores de base de um mesmo ponto material em duas configurações distintas (Holzapfel, 2000). No entanto, o gradiente da transformação é invariante a translação de corpo rígido, mas não pela sua rotação. O F pode ser decomposto através da decomposição polar para obter os tensores dos estiramentos direito $U$, esquerdo $V$ e de rotação $R$ :

$$
F=R U=V R \text {. }
$$


onde $\boldsymbol{R}$ é um tensor ortogonal que possuí as seguintes propriedades:

$$
\boldsymbol{R}^{\top}=\boldsymbol{R}^{-1}, \quad \boldsymbol{R}^{\top} \boldsymbol{R}=\boldsymbol{I}, \quad \operatorname{det}(\boldsymbol{R})=+1
$$

Uma medida de deformação muito utilizada que é invariante as rotações de corpo rígido é o tensor direito de Cauchy-Green, dado por:

$$
\boldsymbol{C}=\boldsymbol{F}^{\top} \boldsymbol{F}=\sqrt{\boldsymbol{U}}=\left(f_{\mathrm{i}} \cdot f_{\mathrm{j}}\right)\left(\boldsymbol{e}_{\mathrm{i}}^{\mathrm{r}} \otimes \boldsymbol{e}_{\mathrm{j}}^{\mathrm{r}}\right)
$$

A partir do tensor direito Cauchy-Green é possível obter o tensor das deformações de Green-Lagrangian ou Green-St-Venant:

$$
\boldsymbol{E}=\frac{1}{2}(\boldsymbol{C}-\boldsymbol{I})=\frac{1}{2}\left(\left(f_{\mathrm{i}} \cdot f_{j}\right)\left(\boldsymbol{e}_{\mathrm{i}}^{\mathrm{r}} \otimes \boldsymbol{e}_{\mathrm{j}}^{\mathrm{r}}\right)-\left(\boldsymbol{e}_{\mathrm{k}}^{\mathrm{r}} \otimes \boldsymbol{e}_{\mathrm{k}}^{\mathrm{r}}\right)\right)
$$

Outro tensor de deformação importante o tensor esquerdo de Cauchy-Green, dado por:

$$
B=F F^{\top}=\sqrt{V}=\left(f_{i} \otimes f_{i}\right) .
$$

Utilizando as componentes $f_{i}$ do gradiente da transformação apresentado na equação (2.2) ao qual tem relação com os estiramentos sofridos por um ponto material, podemos obter a relação da transformação do volume na configuração atualizada:

$$
J=f_{1} \cdot\left(f_{2} \times f_{3}\right)=\operatorname{det}(F)
$$

desse modo $J$ relaciona a configuração de referência $d v^{r}$ em relação a configuração atualizada $d v=\operatorname{det}(\boldsymbol{F}) d v^{\mathrm{r}}$ de um ponto material no espaço. É uma condição suficiente na transformação entre às duas configurações para que o sólido não penetre nele mesmo, portanto, o determinante do gradiente da transformação deve ser positivo em qualquer ponto material dentro do domínio de projeto $\Omega$. Esta condição é denominada por condição local de impenetrabilidade $J>0$ e é uma das condições necessárias para uma lei material policonvexa.

Observe que as transformações preservam o volume em cada ponto material infini- 
tesimal dentro do domínio de projeto $\Omega$ somente se:

$$
J(\boldsymbol{u})=1, \quad \forall \boldsymbol{u},
$$

sendo que tal transformação é denominada isocórica ou isovolumétrica. Um material incompressível é um material que preserva o volume em cada ponto material infinitesimal, ou seja, um material que admite uma transformação isocórica.

Outro conceito importante se dá entre a área de um elemento infinitesimal na configuração de referência $d a^{r}$ e a sua configuração atual $d a$. Esta relação é definida por:

$$
\boldsymbol{n} d a=\operatorname{cof}(\boldsymbol{F})\left(\boldsymbol{n}^{\mathrm{r}} d a^{\mathrm{r}}\right)
$$

sendo conhecida como relação de Nanson.

A matriz de cofatores é apresentada pela fórmula $\operatorname{cof}(\boldsymbol{F})=J \boldsymbol{F}^{-\mathrm{T}}$, tal que:

$$
\operatorname{cof}(\boldsymbol{F}) \boldsymbol{F}=\operatorname{adj}(\boldsymbol{F})^{\top} \boldsymbol{F}=n \operatorname{det}(\boldsymbol{F}) \boldsymbol{I},
$$

no qual $n$ se refere a dimensão do espaço. Um conjunto de vetores que será importante depois são os que representam deformações de áreas, representados por:

$$
g_{i}=\frac{1}{2} \epsilon_{i j k} f_{j} \times f_{k}=\frac{1}{2} \epsilon_{i j k} x_{, j} \times x_{, k}
$$

onde $\epsilon_{i j k}$ se refere a um operador de permutação Levi-Civita, que neste caso opera nas três dimensões:

$$
\epsilon_{i j k}= \begin{cases}+1 & \text { se }(i, j, k) \text { então }(1,2,3),(2,3,1) \text { ou }(3,1,2) \\ -1 & \text { se }(i, j, k) \text { então }(3,2,1),(1,3,2) \text { ou }(2,1,3) \\ 0 & \text { se } i=j, \text { ou } j=k, \text { ou } k=i\end{cases}
$$

Portanto aplicando o operador de permutação (2.13) em (2.12), obtemos:

$$
g_{1}=f_{2} \times f_{3}, \quad g_{2}=f_{3} \times f_{1}, \quad g_{3}=f_{1} \times f_{2} .
$$


Lembrando que a seguinte propriedade de $g_{i} \cdot f_{j}=\delta_{i j} J$, demonstrando assim que $\operatorname{cof}(\boldsymbol{F})=\boldsymbol{e}_{\mathrm{i}}^{\mathrm{r}} \otimes \boldsymbol{g}_{\mathrm{i}}$, já que

$$
\boldsymbol{e}_{i}^{r} \otimes g_{i}\left(f_{j} \otimes \boldsymbol{e}_{j}^{r}\right)=\left(g_{i} \cdot f_{j}\right)\left(\boldsymbol{e}_{i}^{r} \otimes \boldsymbol{e}_{i}^{r}\right)=\delta_{i j} J\left(\boldsymbol{e}_{i}^{r} \otimes \boldsymbol{e}_{i}^{r}\right)=J \boldsymbol{I} .
$$

\subsection{Equações Cinemáticas dos Sólidos Deformáveis}

As equações globais do movimento de translação de uma sólido deformável no espaço na configuração de referência $(\bullet)^{\mathrm{r}}$ é dada pela primeira lei de movimento de Euler:

$$
\Pi_{p}=\int_{\Omega^{r}} \boldsymbol{b}^{r} d v^{r}+\int_{\partial \Omega^{r}} t^{r} d a^{r}-\int_{\Omega^{r}} m^{r} \ddot{u} d v^{r},
$$

no qual os deslocamentos e as condições de contorno superficiais são apresentadas por:

$$
\boldsymbol{u}=\boldsymbol{u} \in \mathcal{H}_{1} \mid \overline{\boldsymbol{u}} \text { em } \partial \Omega_{u^{\prime}}^{\mathrm{r}} \quad \boldsymbol{P} \boldsymbol{n}^{\mathrm{r}}=\boldsymbol{t}^{\mathrm{r}} \text { em } \partial \Omega_{\sigma^{\prime}}^{\mathrm{r}}
$$

onde é o espaço de Sobolev de ordem k para campos escalares, vetoriais ou tensoriais, definidos no domínio $\Omega^{r}$ que possuem derivada fraca (Hughes, 2012). Salientando que as equações globais do movimento apresentadas em (2.17) também referidas como Forma Forte do equilíbrio de uma sólido deformável no espaço na configuração de referência.

A Forma Fraca na configuração atual pode ser obtida aplicando o Princípio dos Trabalhos Virtuais nas equações globais do movimento (2.16) e aplicando o teorema do Divergente obtemos:

$$
\delta \Pi_{p}(\rho, \boldsymbol{u}, \delta \boldsymbol{u})=\int_{\partial \Omega^{r}}\left(\operatorname{div} \boldsymbol{P}+\boldsymbol{b}^{\mathrm{r}}-m^{\mathrm{r}} \ddot{\boldsymbol{u}}\right) \cdot \delta \boldsymbol{u} d v^{\mathrm{r}},
$$

onde $\delta \boldsymbol{u}$ são referente aos vetores dos Deslocamentos Virtuais ou também interpretados como função de teste $\delta \boldsymbol{u}=\left\{\delta \boldsymbol{u} \mid \delta \boldsymbol{u}=\boldsymbol{0}\right.$ em $\left.\partial \Omega_{\mathfrak{u}}^{r}\right\}$. Aplicando a transformação $\operatorname{div}(\boldsymbol{P}) \cdot \delta \boldsymbol{u}$ em (2.18) e o teorema de Gauss considerando o processo conservativo e quase-estático $\ddot{u}=0$ :

$$
\delta \Pi_{p}(\rho, \boldsymbol{u}, \delta \boldsymbol{u})=\delta \mathrm{U}_{\text {int }}(\rho, \boldsymbol{u}, \delta \boldsymbol{u})+\delta \mathrm{U}_{\text {ext }}(\delta \boldsymbol{u})=0, \quad \forall \delta \boldsymbol{u},
$$


tal que $\delta u_{\text {int }}$ e $\delta u_{\text {ext }}$

$$
\begin{aligned}
\delta \mathrm{U}_{\text {int }}(\rho, \boldsymbol{u}, \delta \boldsymbol{u}) & =\int_{\Omega^{r}} \boldsymbol{P}: \delta \boldsymbol{F} d v^{\mathrm{r}}, \\
\delta \mathrm{U}_{\text {ext }}(\boldsymbol{u}, \delta \boldsymbol{u}) & =\int_{\partial \Omega^{\mathrm{r}}} \boldsymbol{t}^{\mathrm{r}} \cdot \delta \boldsymbol{u} d a^{\mathrm{r}}+\int_{\Omega^{\mathrm{r}}} \boldsymbol{b}^{\mathrm{r}} \cdot \delta \boldsymbol{u} d v^{\mathrm{r}},
\end{aligned}
$$

no qual $\delta U_{\text {int }}: \mathcal{U}_{k} \rightarrow \mathbb{R}$ é a trabalho virtual interno e $\delta U_{\text {ext }}: \mathcal{U}_{k} \rightarrow \mathbb{R}$ é a trabalho virtual externo. Ressaltando que dentro das equações de equilíbrio do sistema mecânico (2.19) estão implícitas a dependência do modelo de material $(\bullet)(\boldsymbol{\rho}, \boldsymbol{u})$, portanto, as variáveis que forem representadas por $(\bullet)^{0}$ tem dependência explicita, como, por exemplo, $\boldsymbol{\rho}(\bullet)^{0}$.

A função da energia de deformação específica é dada por:

$$
\delta \mathrm{U}_{\text {int }}(\boldsymbol{\rho}, \boldsymbol{u}, \delta \boldsymbol{u})=\delta\left(\int_{\Omega^{r}} \boldsymbol{\rho}^{p} \varphi^{0}(\boldsymbol{u}) d v^{r}\right)=\int_{\Omega^{r}} \boldsymbol{\rho}^{p} \frac{\partial \varphi^{0}}{\partial \boldsymbol{F}}: \delta \boldsymbol{F} d v^{\mathrm{r}}
$$

onde $\varphi$ é uma função referente a uma lei material hiperelástica $\varphi^{0}(\boldsymbol{u}): \mathcal{U}_{k} \times \mathbb{R} \rightarrow \mathbb{R}$, na qual a teoria da hiperelasticidade postula a existência de uma função de energia livre de Helmholtz $\varphi$ definida por unidade de volume $d v^{r}$ ao invés da unidade de massa para um processo reversível ao qual a dissipação da energia interna é zero (sem a geração de entropia), portanto, um processo conservativo (Holzapfel, 2000; Bertram, 2005). Logo por definição (Ciarlet, 1988b) temos a seguinte relação entre o par energeticamente conjugado $\{\boldsymbol{P}: \boldsymbol{F}\}$ :

$$
\boldsymbol{P}^{0}=\frac{\partial \varphi^{0}(\boldsymbol{F})}{\partial \boldsymbol{F}}=\boldsymbol{\tau}_{i}^{0} \otimes \boldsymbol{e}_{i}^{r}
$$

onde $\boldsymbol{P}^{0}$ é o tensor das tensões nominais, de engenharia ou o primeiro tensor das tensões de Piola Kirchhoff[PK1, Como $\boldsymbol{P}^{0}$ é um tensor de campo de dois pontos pode ser decomposto pelas suas componentes $\tau_{\mathfrak{i}}^{0}$ da mesma maneira que o tensor do gradiente das transformações $\boldsymbol{F}$.

A partir da equação (2.20) é possível obter o problema em relação as variáveis primais $(\boldsymbol{u})$ em seu respectivo par energeticamente conjugado decomposto em relação as componentes de base na configuração de referência $\boldsymbol{e}_{i}^{r}$ dado pelas equações (2.2) e 
(2.22) respectivamente:

$$
\begin{aligned}
\delta \Pi_{p}(\boldsymbol{\rho}, \boldsymbol{u}, \delta \boldsymbol{u}) & =\int_{\Omega^{r}} \boldsymbol{\rho} \boldsymbol{\tau}_{i}^{0} \cdot \delta \gamma_{i} d v^{r}-\left(\int_{\partial \Omega^{r}} \boldsymbol{t}^{\mathrm{r}} \cdot \delta \boldsymbol{u} d a^{\mathrm{r}}+\int_{\Omega^{r}} \boldsymbol{b}^{\mathrm{r}} \cdot \delta \boldsymbol{u} d v^{\mathrm{r}}\right) \\
& =\int_{\Omega^{r}} \boldsymbol{\rho}^{p} \frac{\partial \varphi^{0}}{\partial \boldsymbol{F}}: \delta \boldsymbol{F} d v^{r}-\left(\int_{\partial \Omega^{r}} \boldsymbol{t}^{\mathrm{r}} \cdot \delta \boldsymbol{u} d a^{r}+\int_{\Omega^{r}} \boldsymbol{b}^{r} \cdot \delta \boldsymbol{u} d v^{r}\right) .
\end{aligned}
$$

A função do trabalho externo para o caso conservativo, onde não é considerada a dependência das pseudo-densidades é dada por:

$$
\mathrm{U}_{\text {ext }}(\boldsymbol{u})=\int_{\partial \Omega^{\mathrm{r}}} \boldsymbol{t}^{\mathrm{r}} \cdot \boldsymbol{u} d a^{\mathrm{r}}+\int_{\Omega^{\mathrm{r}}} \boldsymbol{b}^{\mathrm{r}} \cdot \boldsymbol{u} d v^{\mathrm{r}}
$$

Complementando as informações sobre tensores de tensão, o segundo tensor das tensões de Piola Kirchhoff $S \overline{\text { PK2 }}$ é definido por:

$$
S^{0}=\boldsymbol{F}^{-1} \boldsymbol{P}^{0}
$$

e seu par energeticamente conjugado é expresso por $\{\boldsymbol{S}: \boldsymbol{E}\}$. O tensor das tensões de Cauchy ou tensor das tensões na configuração atualizada é dada por:

$$
T^{0}=P^{0} \frac{F^{\top}}{J},
$$

onde seu par energético conjugado é referente a parte simétrica do gradiente da velocidade $\boldsymbol{D}=\operatorname{Sym}(\nabla \boldsymbol{v})$, dado por:

$$
\boldsymbol{v}=\frac{d x}{d t}, \quad \boldsymbol{D}=\frac{1}{2}\left(\nabla \boldsymbol{v}^{\top}+\nabla \boldsymbol{v}\right)
$$




\subsection{Princípio da Objetividade Material}

O princípio da objetividade estabelece que uma lei de material deva ser invariante perante a superposição de movimentos de corpos rígidos ao movimento de um sólido ou da mudança de observador. Portanto, a lei material deve ser objetiva (ou neutra) em relação à transformação Euclidiana do movimento superpostos de corpo rígido (equação 2.28). Fisicamente o que se deseja é que os esforços internos não sejam afetados por movimentos de corpo rígido, pois, estes não provocam deformações, portanto, as propriedades materiais não podem depender da mudança do observador (Pimenta, 2008; Bertram, 2005; Holzapfel, 2000).

Um exemplo do princípio da objetividade é dado pelo movimento de um sólido no espaço descrito por $x\left(x^{r}, t\right)$. Superpor um movimento de corpo rígido a este movimento significa a seguinte transformação Euclidiana:

$$
x^{*}\left(x^{\mathrm{r}}, t\right)=x\left(x_{0}^{\mathrm{r}}, t\right)+q_{0}(t)+Q(t)\left(x\left(x^{\mathrm{r}}, t\right)-x\left(x_{0}^{\mathrm{r}}, t\right)\right),
$$

onde $Q(t)$ é um tensor que impõem um movimento de rotação superposto e $q_{0}(t)$ é um vetor que impõem um movimento de translação adicional do ponto 0 .

Podemos classificar funções escalares, vetoriais e tensoriais em objetivas e invariantes (Bertram, 2005; Holzapfel, 2000). Uma função é objetiva quando a sua transformação é dada por:

$$
\begin{aligned}
& \phi^{*}\left(x^{r}, t\right)=\phi\left(x^{r}, t\right), \\
& b^{*}\left(x^{r}, t\right)=Q b\left(x^{r}, t\right), \\
& A^{*}\left(x^{r}, t\right)=Q A\left(x^{r}, t\right) Q^{\top} .
\end{aligned}
$$

Uma função é invariante quando a sua transformação é neutra em relação a imposição 
de movimentos superpostos de corpo rígido, esta transformação é dada por:

$$
\begin{aligned}
& \phi^{*}\left(x^{r}, t\right)=\phi\left(x^{r}, t\right), \\
& b^{*}\left(x^{r}, t\right)=b\left(x^{r}, t\right), \\
& A^{*}\left(x^{r}, t\right)=A\left(x^{r}, t\right),
\end{aligned}
$$

onde é possível observar que a função escalar $\phi^{*}$ é invariante e também objetiva.

Recorrendo a equação do movimento do sólido (2.28), um novo gradiente da transformação é obtido pela diferenciação segundo a sua posição na configuração de referência $x^{\mathrm{r}}$, o que resulta em:

$$
\boldsymbol{F}^{*}\left(\boldsymbol{x}^{*}\right)=\frac{\partial \boldsymbol{x}^{*}}{\partial x} \frac{\partial x}{\partial \boldsymbol{x}^{\mathrm{r}}}=\boldsymbol{Q} \boldsymbol{F}, \quad \therefore \quad \operatorname{det}\left(\boldsymbol{F}^{*}\right)=\operatorname{det}(\boldsymbol{Q}) \operatorname{det}(\boldsymbol{F})=J,
$$

no entanto o gradiente da transformação é afetado por movimentos superpostos de corpo rígido, como exposto pela equação (2.31), porém é um tensor objetivo mesmo que a sua transformação seja a mesma de um vetor e não como a transformação de tensor de segunda ordem (equação (2.29)). Isto ocorre porque uma parte do tensor $\boldsymbol{F}$ descreve as coordenadas materiais ou de referência que são independentes do observador, ou seja é um tensor de campo de dois pontos ou um vetor duplo (Holzapfel, 2000). Baseado nesta propriedade o novo gradiente da transformação $\left(\boldsymbol{F}^{*}\right)$ é utilizado para descrever o novo tensor das deformações de Green com movimento de corpo rígido superposto:

$$
\boldsymbol{E}^{*}=\frac{1}{2}\left(\boldsymbol{F}^{* \top} \boldsymbol{F}^{*}-\boldsymbol{I}\right)=\frac{1}{2}\left(\boldsymbol{F}^{\top} \boldsymbol{Q}^{\top} \boldsymbol{Q} \boldsymbol{F}-\boldsymbol{I}\right)=\frac{1}{2}\left(\boldsymbol{F}^{\top} \boldsymbol{F}-\boldsymbol{I}\right)=\boldsymbol{E},
$$

como o tensor $Q$ é ortogonal e cujas propriedades estão descritas em (2.4), a imposição de movimento de corpo rígido superposto não afeta o tensor de Green $E$ (eq. 2.6). Podemos concluir então que o tensor direito de Cauchy $C$ (eq. 2.5) também não é afetado, logo, ambos os tensores são Invariantes ao movimento de corpo rígido.

Para verificar os demais tensores de tensão em relação a objetividade e invariança à superposição de movimentos de corpo rígido é necessário recorrer ao teorema das tensões de Cauchy (Bertram, 2005; Holzapfel, 2000; Bonet e Wood, 1997), onde o vetor 
normal a uma superfície $\boldsymbol{n}$ e o vetor das força superficiais $\boldsymbol{t}$ ambos na configuração atualizada são objetivos e por consequência o tensor das tensões de Cauchy também é objetivo conforme transformação (2.29).

A verificação da objetividade do Primeiro Tensor das Tensões de Piola Kirchhoff (PK1) $\boldsymbol{P}$ (eq. 2.22) pode ser demonstrada recorrendo ao tensor das tensões de Cauchy $\boldsymbol{T}$ :

$$
\begin{aligned}
\boldsymbol{P}^{*}(\boldsymbol{Q F})^{\top} & =J \boldsymbol{Q T} \boldsymbol{Q}^{\top} \\
\boldsymbol{P}^{*}\left(\boldsymbol{Q}^{\top} \boldsymbol{F}^{\top}\right)^{\top} & =J \boldsymbol{Q T} \boldsymbol{Q}^{\top}=\boldsymbol{Q P}\left(\boldsymbol{Q}^{\top} \boldsymbol{F}^{\top}\right) \\
\boldsymbol{P}^{*} & =\boldsymbol{Q P},
\end{aligned}
$$

em uma primeira análise o tensor $\boldsymbol{P}$ não é objetivo e nem invariante Bertram (2005); Altenbach (2012), porém, se analisarmos em mais detalhes a sua composição, verificaremos que se trata de um tensor de campo de dois pontos do mesmo tipo do gradiente da transformação $\boldsymbol{F}$, portanto, $\boldsymbol{P}$ deve ser verificado como um vetor (2.29), consequentemente $\boldsymbol{P}$ é um tensor objetivo e não invariante ao movimento de corpo rígido Holzapfel (2000).

A verificação do Segundo Tensor das Tensões de Piola Kirchhoff (ㅈ2) $S$ (eq. 2.25) é dada por:

$$
\boldsymbol{S}^{*}=\boldsymbol{F}^{*-1} \boldsymbol{P}^{*}=\boldsymbol{F}^{-1} \boldsymbol{Q}^{\top} \boldsymbol{Q P}=\boldsymbol{F}^{-1} \boldsymbol{P}=S,
$$

logo o tensor $S$ é invariante à superposição de movimentos de corpo rígido.

Uma forma simples de satisfazer o Princípio da Objetividade é expressar algum tensor não afetado pelo movimento de corpo rígido em função de algum tensor das deformações que também não seja afetado pelo movimento de corpo rígido Bertram (2005); Pimenta (2008). Por este motivo é importante utilizar tensores invariantes nas equações constitutivas, sendo esta uma das razões para a utilização do par energeticamente conjugado $\{\boldsymbol{S}, \boldsymbol{E}\}$ na análise não linear geométrica Bonet e Wood (1997); Wriggers (2008). Qualquer outro par conjugado é permitido, desde que o Princípio da Objetividade seja respeitado. Mesmo que o par conjugado em si não seja invariante $\{\boldsymbol{P}, \boldsymbol{F}\}$, a lei de material descrita por este par deve ser composta por parâmetros tensoriais invariantes. 
A verificação da objetividade e invariância da função energia de deformação específica $\varphi$ é equivalente a verificação da objetividade e invariância dos pares energeticamente conjugados (Bertram, 2005; Holzapfel, 2000), portanto a função energia de deformação específica deve obedecer a seguinte restrição:

$$
\varphi(\boldsymbol{F})=\varphi\left(\boldsymbol{F}^{*}\right)=\varphi(\boldsymbol{Q F})
$$

onde $\boldsymbol{F}$, det $(\boldsymbol{F})>0$ para qualquer tensor ortogonal $Q$, $\operatorname{det}(\boldsymbol{Q})=+1$, desde que a transformação $F=Q F$ seja respeitada.

\subsection{Policonvexidade}

Uma função de energia de deformação policonvexa $\varphi(\boldsymbol{F})$ implica que o seu respectivo tensor acústico material é elíptico para todas as possíveis deformações, garantindo que o material seja estável e que possua um estado de deformação que minimiza $\Pi_{p}$, ou seja, representa uma condição necessária para a existência de solução. Uma vantagem de uma função policonvexa $\varphi(\boldsymbol{F})$ é não ser necessariamente convexa em relação ao tensor $\boldsymbol{F}, \log$ o não é uma condição suficiente para a existência de um mínimo global (Ciarlet, 1988a; Ball, 1976).

Uma outra forma de garantir uma condição suficiente para a existência de solução é utilizar uma função de energia de deformação $\varphi$ estritamente convexa em $\boldsymbol{F}$, garantindo uma singularidade da solução, o que significa que um mínimo local é sempre um mínimo global. É uma condição muito restrita, pois, pode excluir alguns fenômenos físicos como flambagem (Ball, 1976) e também contradiz o Princípio da Obietividade (Coleman e Noll, 1959). Isto leva a um importante conceito de quase convexidade (quasiconvexity) introduzido por Morrey et al. (1952), onde uma função $\varphi(F)$ é quase convexa se:

$$
\int_{\Omega^{r}} \varphi(\boldsymbol{F}+\nabla \boldsymbol{u}) d v^{r} \geqslant \int_{\Omega^{r}} \varphi(\boldsymbol{F}) d v^{r},
$$

sendo uma condição necessária e suficiente para o funcional ser weakly lower semi- 
continuous, ou seja, é uma condição menos restrita que a condição de convexidade. Essa desigualdade é equivalente a dizer que o estado homogêneo de deformação é o mínimo do funcional $\Pi_{p}$, protegendo-o de instabilidades materiais. Infelizmente a quase convexidade é uma condição de desigualdade difícil de ser verificada, além disso a condição de crescimento exclui o tratamento da energia $\varphi(\boldsymbol{F}) \rightarrow+\infty$ quando $\operatorname{det}(\boldsymbol{F}) \rightarrow 0$, sendo um importante conceito relacionado a grandes deformações.

Um conceito de maior importância prática é o da policonvexidade. Segundo Marsden e Hughes (1994), uma função $\varphi$ é policonvexa se e somente se existir uma função $\psi$ composta pelos argumentos $F, \operatorname{cof} F$ e $\operatorname{det} F$, conforme apresentado a seguir:

$$
\varphi(\boldsymbol{F})=\psi(\boldsymbol{F}, \operatorname{cof} \boldsymbol{F}, \operatorname{det} \boldsymbol{F})=\psi_{1}(\boldsymbol{F})+\psi_{2}(\operatorname{cof} \boldsymbol{F})+\psi_{3}(\operatorname{det} \boldsymbol{F}),
$$

se $\psi_{i}, i=1, \ldots, 3$ é convexa em relação a um dos seus argumentos, então a função $\varphi(\boldsymbol{F})$ é policonvexa. A verificação aditiva é muito útil para conceber funções que não seja sujeitas a instabilidade material.

Uma outra opção para a verificação da estabilidade material é a condição de elipticidade que estabelece uma função de energia de deformação específica $\varphi(\boldsymbol{F})=\varphi(\boldsymbol{C})$ diferenciável duas vezes conduzindo a um sistema de equilíbrio uniformemente elíptico sempre que chamada a condição uniforme de Legendre-Hadamard. A perda de elipticidade ocorre quando o determinante do tensor acústico de $\varphi(\boldsymbol{F})$ torna-se zero, levando a uma condição de equilíbrio com um campo de deslocamento contínuo, mas com derivadas temporais descontínuas em relação ao gradiente da transformação $\boldsymbol{F}$. Neste caso, o material é dito instável (Kuhl et al., 2006).

Portanto, uma função de policonvexa também é uma função elíptica, uma função policonvexa suave em relação ao tensor $\boldsymbol{F}$, o que também implica que a condição de Legendre-Hadamard seja satisfeita, logo, podemos listar a ordem de verificação de estabilidade material da seguinte forma: 
Convexidade ${ }_{\notin}^{\Rightarrow}$ policonvexidade $\underset{\Leftarrow \text { quasi convexidade }}{\Rightarrow}$ elipticidade.

\subsection{Lei de Material de neo-Hookiano de Simo-Ciarlet}

Nesta seção é apresentado o modelo hiperelásticos utilizado neste trabalho para descrever o comportamento elástico de um sólido no espaço. Foi adotado um modelo isótropo baseado nas constantes de propriedades de material de Lamé $(\lambda, \mu)$, que representam as propriedades físicas intrínsecas de uma dado material, determinando o estado de tensões em qualquer ponto material para uma transformação arbitrária $\boldsymbol{F}$.

A lei material neo-Hookiano é um modelo hiperelástico semelhante à lei de material de Lei Material Kirchhof Saint Venant ( $(\mathrm{KSV})$ com característica isótropa. É baseada na termodinâmica estatística das cadeias longas de polímeros reticulados e foi proposta inicialmente por Treloar (1943), possuindo apenas dois parâmetros constitutivos $(\lambda, \mu)$. É muito utilizada para representar o comportamento de materiais plásticos e borrachas, tendo sido muito estudada e aprimorada ao longo dos anos (Ciarlet, 1988b; Simo e Hughes, 1991; Bonet e Wood, 1997; Holzapfel, 2000). Neste trabalho foi utilizada a modificação proposta por Ciarlet (1988a); Simo e Hughes (1991), sua introdução no MOT foi sugerida por Penzler et al. (2012); Lahuerta et al. (2013) com a finalidade de demonstrar a sua importância na estabilização dos elementos de baixa densidade durante o processo de otimização evitando instabilidade material, como relatado pelo trabalho de Buhl et al. (2000).

A função da energia de deformação específica da lei de material de Lei Material neo-Hookiana de Simo-Ciarlet $(\underline{\mathrm{nH}})$ é dada por:

$$
\varphi^{0}\left(\mathrm{I}_{1}^{\mathrm{C}}, J\right)=\mu \mathrm{A}\left(\mathrm{I}_{1}^{\mathrm{C}}, J\right)+\lambda \mathrm{U}(J)
$$

onde $A=0.5\left(\mathrm{I}_{1}^{\mathrm{C}}-n-2 \ln J\right), \mathrm{U}(J)=0.25\left(J^{2}-1-2 \ln J\right)$ e $\mathrm{I}_{1}^{\mathrm{C}}=\operatorname{tr} C$ é referente ao tensor direito de Cauchy. A variação de volume $J^{2}$ também pode ser chamada de terceiro 
invariante do tensor direito de Cauchy representado por $\mathrm{I}_{3}^{\mathrm{C}}$.

É uma lei material que obedece o princípio da Objetividade como apresentado na seção 2.3, $\operatorname{logo} \varphi^{0}(\boldsymbol{F})=\varphi^{* 0}(\boldsymbol{Q F})$ satisfazendo a restrição de Invariância, pois $\operatorname{det}(\boldsymbol{F})=$ $\operatorname{det}(\boldsymbol{Q F})$ e $C(\boldsymbol{F})=C^{*}(\boldsymbol{Q F})$.

É importante também definir a primeira variação da função de energia de deformação (2.38) em relação ao gradiente da transformação, para obter o tensor de engenharia PK1, dado por:

$$
\frac{\partial \varphi^{0}}{\partial \boldsymbol{F}}=\boldsymbol{P}^{0}=\boldsymbol{\tau}_{\mathrm{i}}^{0} \otimes \boldsymbol{e}_{\mathrm{i}}^{\mathrm{r}}=\left(\frac{\lambda}{2}\left(J^{2}-1\right)-\mu\right) \frac{\boldsymbol{g}_{\mathrm{i}}}{J} \otimes \boldsymbol{e}_{\mathrm{i}}^{\mathrm{r}}+\mu\left(\boldsymbol{f}_{\mathrm{i}} \otimes \boldsymbol{e}_{\mathrm{i}}^{\mathrm{r}}\right)
$$




\section{Capítulo 3}

\section{Formulação Numérica}

Para obter a solução de estacionariedade do equilíbrio quase-estático $\left(\delta \Pi_{p}\left({ }^{*} \boldsymbol{u}\right)=\boldsymbol{0}\right)$ no espaço ${ }^{*} x$ é necessário aplicar um método de solução numérica para que seja possível estimar o vetor das variáveis primais * $\boldsymbol{u}$ (deslocamentos), no entanto, é fundamental obter a linearização da forma fraca do variacional $\Delta\left(\delta \Pi_{p}\right)$ ou a aproximação de $\delta^{2} \Pi_{p}$ do problema. O método de Newton pode ser aplicado para esta finalidade, pois, é um método muito eficiente, no entanto, não é capaz de obter múltiplas soluções de equilíbrio em problemas de instabilidade. Portanto, neste Capítulo será descrita a linearização de $\delta \Pi_{p}$ com o objetivo de descrever os Métodos de Newton, Comprimento de Arco e do Controle Generalizado dos Deslocamentos. Na sequência será descrito o método de otimização numérico aplicado para obter a ótima distribuição de material * $\rho$ ao longo do domínio de projeto via $\mathrm{MOT}$

\subsection{Ambiente de Implementação}

Utilizando a forma variacional apresentada em (2.23) é possível implementar um código para solucionar as EDPsutilizando o MEFe o MOT, no entanto, esta implementação pode ser feita manualmente no ambiente de programação do $M A T L A B$, da linguagem Python ou de forma automatizada, utilizando o método de derivação automatizado 
(Griewank et al., 1996) para calcular todas as derivadas e variações de um dado funcional necessário para descrever o equilíbrio do sistema $\Pi_{p}$ e obter as EDPs,

Neste trabalhou utilizou-se a plataforma FEniCS (Logg et al., 2012) para obter as EDPS de forma automatizada, no entanto, os métodos utilizados para a solução do equilíbrio ou pontos estacionários são descritos neste Capítulo (ver Seção 3.5).

A plataforma|FEniCSé uma coleção de software e módulos que automatizam a geração e solução das EDPs. Este processo automatizado envolve a tradução de linguagem matemática simbólica (Unified Form Language (UFL)) que é interpretada em um código C++ compilável (FEniCS Form Compiler (파) ) que contém todas as funções otimizadas necessárias para obter as EDPs,

Para solucionar as EDPs, são utilizados os tensores obtidos pelo método de derivação automatizado que são interpolados via MEFe estas equações podem ser resolvidas por diversas biblioteca de álgebra linear, como PETSc, LAPACK, $u B L A S$ e também combinálas ao método de Newton ou Picard (Logg et al., 2012).

\subsection{Linearização da Forma Fraca}

A forma fraca do problema primal (2.23) é composta por expressões não lineares em suas respectivas relações cinemáticas, onde a linearização irá permitir gerar um sistema de equações (ou $\overline{E D P S}$ ) tangente que possibilitará obter a estimativa das variáveis primais $\left({ }^{*} \boldsymbol{u}\right)$ dentro de uma determinada tolerância de erro ou de convergência " $\epsilon$ "baseada na norma $L^{2}$.

Aplicando a linearização na forma fraca do equilíbrio (2.23) por:

$$
\Delta\left(\delta \Pi_{p}\right)=\hat{\Pi}_{p}(\rho, u, \delta u)+\Delta \hat{\Pi}_{p}(\rho, u, \delta u, \Delta u)
$$


na qual a forma fraca incremental do equilíbrio $\Delta \hat{\Pi}_{p}$ é obtida pela derivada direcional definida por:

$$
\Delta \hat{\Pi}_{p}(\rho, u, \delta u, \Delta u)=\left.\frac{d}{d \epsilon}\left[\delta \Pi_{p}(u+\Delta u, \delta u)\right]\right|_{\epsilon=0}=D \hat{\Pi}_{p}(\rho, u, \delta u) \cdot \Delta u
$$

onde $\hat{\Pi}_{p}(\rho, u, \delta \boldsymbol{u})$ é referente ao resíduo do trabalho virtual.

$$
\Delta \hat{\Pi}_{p}=\Delta \int_{\Omega^{r}}\left(\boldsymbol{\tau}_{i} \cdot \delta \gamma_{i}-\boldsymbol{b}^{r} \cdot \delta \boldsymbol{u}\right) d v^{r}-\Delta \int_{\partial \Omega^{r}} \boldsymbol{t}^{r} \cdot \delta \boldsymbol{u} d a^{r}
$$

Expandindo a linearização $\Delta \hat{\Pi}_{p}$ nos termos internos de cada integral de área e volume obtemos:

$$
\Delta \hat{\Pi}_{p}=\int_{\Omega^{r}}\left(\delta \gamma_{i} \cdot C_{i j} \Delta \gamma_{j}-\delta \boldsymbol{u} \cdot L_{v} \Delta u\right) d v^{r}-\int_{\partial \Omega^{r}} \delta \boldsymbol{u} \cdot \boldsymbol{L}_{s} \Delta \boldsymbol{u} d a^{r}
$$

onde os tensores $\frac{\partial \tau_{i}}{\partial \gamma_{j}}=C_{i j}, \frac{\partial b^{r}}{\partial u}=L_{v}, \frac{\partial t^{r}}{\partial u}=L_{s}$ são denominados de tensores tangente material, das forças de volume, das forças de superfície respectivamente. Iremos representar estes tensores por letras conforme a notação matemática para diferenciar a sua respectiva ordem tensorial e facilitar o equacionamento que será feito ao longo do texto.

\subsection{Método de Elementos Finitos}

$\mathrm{O}$ MEF é um método numérico de interpolação utilizado neste trabalho para obter a solução do comportamento de um sólido no espaço considerando a sua cinemática e elasticidade finita. A solução é obtida através aproximação da geometria, variáveis primais e equações globais de movimento a partir da aproximação do domínio $\Omega^{r}$ em uma somatória de subdomínios $\Omega_{e}^{r}$.

A forma fraca do problema em questão é obtida pelo princípio dos trabalhos virtuais (Capítulo 2.2), em seguida linearizada (Seção 3.2) e depois introduzida a aproximação por elementos finitos, obtendo um sistema tangente de equações não lineares baseados 
nas variáveis primais do problema $\mathbf{u}$. Geralmente o sistema tangente de equações não lineares são resolvidas utilizando o Método de Newton (Seção 3.5.1)

Neste trabalho foi adotado o elemento Lagrangiano de ordem 1 que satisfaz a condição $C^{1}$ em $\Omega^{r}$, portanto, $u \in \mathcal{H}_{1}$ (Logg et al., 2012; Hughes, 2012). As funções de aproximação ou interpolação dentro de um subdomínio $\Omega_{e}^{r}$ é dada por:

$$
x_{e}=\mathbf{N}_{e} \mathbf{x}_{e}, u_{e}=\mathbf{N}_{e} \mathbf{u}_{e},
$$

onde $\mathbf{N}_{e}$ é referente a matriz com as funções de interpolação elemento $\Omega_{e}^{r}$ e $\mathbf{x}_{e}, \boldsymbol{u}_{e}$ são referente aos vetores de posição e o dos deslocamentos, onde $e=1, \ldots, n_{e}$. As componentes do vetor do gradiente da transformação baseado nas derivadas em relação as coordenadas ou grau de liberdade $i$ são apresentadas por:

$$
\left[\gamma_{i}\right]_{e}=\mathbf{N}_{e, i} \mathbf{u}_{e}
$$

$\log 0 \mathbf{N}_{e, i}$ se refere a matriz das derivadas da função de forma ou de interpolação. Aplicando as funções interpoladoras (3.5) e (3.6) de um elemento e no vetor local do resíduo do variacional linearizado (3.1) obtemos a aproximação do trabalho virtual interno:

$$
\delta\left[\mathrm{u}_{\mathrm{int}}\right]_{e}=\int_{\Omega_{e}^{r}}\left[\boldsymbol{\tau}_{i}^{0}\right]_{e} \cdot \mathbf{N}_{e, i} \delta \mathbf{u}_{e} d v_{e}^{r}
$$

e para o trabalho virtual externo obtemos:

$$
\delta\left[\mathrm{u}_{\mathrm{ext}}\right]_{e}=-\int_{\Omega_{e}^{r}} \boldsymbol{b}_{e}^{\mathrm{r}} \cdot \mathbf{N}_{e} \delta \mathbf{u}_{e} d v_{e}^{r}-\int_{\partial \Omega_{e}^{r}} \boldsymbol{t}_{e}^{\mathrm{r}} \cdot \mathbf{N}_{e} \delta \mathbf{u}_{e} d a_{e^{\prime}}^{r} \quad \forall \delta \mathbf{u}_{e} .
$$

Isolando os termos $\delta \mathbf{u}_{e}$ :

$$
\left[\hat{\Pi}_{p}\right]_{e}=(\underbrace{\int_{\Omega_{e}^{r}} \mathbf{N}_{e, i}^{\top}\left[\boldsymbol{\tau}_{i}\right]_{e} d v_{e}^{r}}_{\left[\mathbf{f}_{f}\right]_{e}}-\underbrace{\int_{\Omega_{e}^{r}} \mathbf{N}_{e}^{\top} \boldsymbol{b}_{e}^{r} d v_{e}^{r}-\int_{\Omega_{e}^{r}} \mathbf{N}_{e}^{\top} \boldsymbol{t}_{e}^{r} d a_{e}^{r}}_{\left[\mathbf{f}_{\mathrm{ext}}\right]_{e}}) \cdot \delta \mathbf{u}_{e}=0 .
$$


Fazendo a somatória ou a montagem do vetor global dos esforços (3.9) em cada elemento finito e que este contenha um nó " $a$ " $(e \ni a)$ dentro do domínio $\left(\Omega^{r}\right)$ é possível obter os vetores globais dos esforços internos e externos:

$$
\sum_{e=1, e \ni a}^{n_{e}} \underbrace{\left[\mathbf{f}_{\text {int }}\right]_{e}^{a}}_{\text {esforços internos }}-\underbrace{\left[\mathbf{f}_{\text {ext }}\right]_{e}^{a}}_{\text {esforços externos }}=\mathbf{f}_{\text {int }}-\mathbf{f}_{\text {ext }}=\mathbf{r}(\rho, \mathbf{u})=\mathbf{0},
$$

onde $\mathbf{r}(\mathbf{u})$ é denominado de vetor do resíduo dos esforços.

Aplicando as aproximações das funções de forma 3.5 em 3.4 obtemos a seguinte aproximação de $\Delta \hat{\Pi}_{p}$ :

$$
\begin{aligned}
{\left[\Delta \hat{\Pi}_{\mathrm{p}}\right]_{e} } & =\delta \mathbf{u}_{e} \cdot \int_{\Omega^{r}} \mathbf{N}_{e, i}^{\top}\left[\boldsymbol{C}_{i j}\right]_{e} \mathbf{N}_{e, j} \Delta \mathbf{u}_{e} d v_{e}^{r}-\delta \mathbf{u}_{e} \cdot \int_{\Omega^{r}} \mathbf{N}_{e}^{\top}\left[\boldsymbol{L}_{v}\right]_{e} \mathbf{N}_{e} \Delta \mathbf{u}_{e} d v_{e}^{r} \\
& -\delta \mathbf{u}_{e} \cdot \int_{\partial \Omega^{r}} \mathbf{N}_{e}^{\top}\left[\boldsymbol{L}_{s}\right]_{e} \mathbf{N}_{e} \Delta \mathbf{u}_{e} d a_{e}^{r}
\end{aligned}
$$

onde cada termos será representado por:

$$
\begin{cases}{\left[\mathbf{K}_{M}\right]_{e}=\int_{\Omega^{r}} \mathbf{N}_{e, i}^{\top}\left[\boldsymbol{C}_{i j}\right]_{e} \mathbf{N}_{e, j} d v_{e}^{r}} & \text { Matriz tangente material. } \\ {\left[\mathbf{K}_{\mathrm{V}}\right]_{e}=\int_{\Omega^{r}} \mathbf{N}_{e}^{\top}\left[\boldsymbol{L}_{v}\right]_{e} \mathbf{N}_{e} d v_{e}^{r}} & \text { Matriz tangente das forças de volume. } \\ {\left[\mathbf{K}_{\mathrm{S}}\right]_{e}=\int_{\partial \Omega^{r}} \mathbf{N}_{e}^{\top}\left[\boldsymbol{L}_{\mathrm{s}}\right]_{e} \mathbf{N}_{e} d a_{e}^{r}} & \text { Matriz tangente das forças de superfície. }\end{cases}
$$

Fazendo a montagem dos vetores e da matriz tangentes globais, obtendo desta forma o sistema tangente de equações linearizadas. Isolando $\delta \mathbf{u}$ o sistema de equações que será solucionado é dado:

$$
\Delta\left(\delta \Pi_{\mathrm{p}}\right)=\left(\left(\mathbf{K}_{\mathrm{M}}+\mathbf{K}_{\mathrm{V}}+\mathbf{K}_{\mathrm{S}}\right) \Delta \mathbf{u}+\mathbf{r}\right) \cdot \delta \mathbf{u}=\left(\mathbf{K}_{\mathrm{T}} \Delta \mathbf{u}+\mathbf{r}\right) \cdot \delta \mathbf{u}=\mathbf{0}
$$

tal que o sistema (3.13) pode ser resolvido numericamente pelo método de Newton, onde, o vetor global do incremento dos deslocamentos $\Delta \mathbf{u}$ é atualizado iterativamente $\mathbf{u}=\mathbf{u}+\Delta \mathbf{u}$ até o valor do vetor do resíduo (3.10) se aproximar de uma tolerância $\epsilon$ desejada (ver Capítulo 3.5.1). 


\subsection{Aproximações Bidimensionais}

Quando um problema de elasticidade tridimensional " $\boldsymbol{u}\left(x_{1}^{r}, x_{2}^{r}, x_{3}^{r}\right)^{\prime \prime}$ é aproximado ou restrito a um problema de duas dimensões, uma das dimensões se tornará dependente das outras duas dimensões independentes " $u\left(\chi_{1}^{r}, \chi_{2}^{r}\right)$ "e para esta condição temos três hipóteses, Estado Plano de Deformação (EPD), Estado Plano de Tensão (EPT) e Axissimetria.

\subsubsection{Estado Plano de Deformação -EPD}

No EPD assume-se que o gradiente da transformação (2.2) a componente 33 não se altera $\left(F_{33}=1\right.$ ou $\left.f_{3}=1\right)$, ou seja, assume-se a hipótese que ao longo da direção 3 $\left(\boldsymbol{e}_{3}^{r}\right)$ o sólido possuí uma espessura muito grande e com uma seção constante sujeita a carregamentos somente nas coordenadas 1 e $2\left(\boldsymbol{e}_{\alpha}^{\mathrm{r}}\right)$ conforme ilustrado pela figura 3.1 respectivamente. Portanto a hipotese EPD aplicada ao gradiente da transformação é

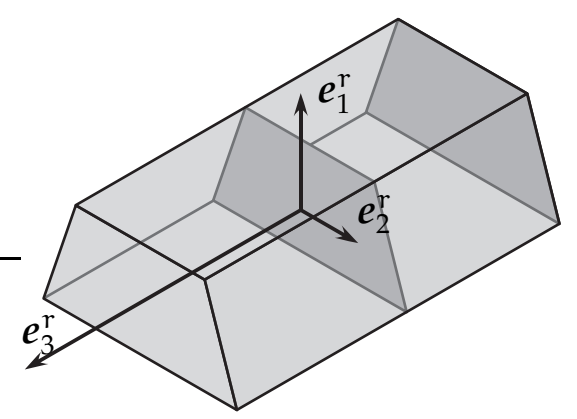

Figura 3.1: Representação da hipótese doEPD $\left(\gamma_{33}=0\right.$ e $\left.\tau_{33} \neq 0\right)$.

dada por:

$$
\boldsymbol{F}^{(\mathrm{D})}=\frac{\partial x_{\alpha}}{\partial x_{\beta}^{r}}\left(\boldsymbol{e}_{\alpha}^{\mathrm{r}} \otimes \boldsymbol{e}_{\beta}^{\mathrm{r}}\right)+\left(\boldsymbol{e}_{3}^{\mathrm{r}} \otimes \boldsymbol{e}_{3}^{\mathrm{r}}\right)=\tilde{\boldsymbol{F}}+\left(\boldsymbol{e}_{3}^{\mathrm{r}} \otimes \boldsymbol{e}_{3}^{\mathrm{r}}\right)=\tilde{\boldsymbol{F}}+\boldsymbol{I}_{3},
$$

acarretando em $\gamma_{33}^{0}=0$ e $\tau_{33} \neq 0$ (Pimenta, 2008).

Aplicando esta hipótese EPD no invariante $\mathrm{I}_{1}(.2)$ é possível obter a mesma decomposição 
feita no gradiente da transformação (3.14)

$$
\mathrm{I}_{1}\left(\boldsymbol{F}^{(\mathrm{D})}\right)=\underbrace{\mathrm{I}_{1}(\tilde{\boldsymbol{F}})}_{\mathrm{I}_{1}^{(\mathrm{D})}}+\underbrace{\mathrm{I}_{1}\left(\boldsymbol{I}_{3}\right)}_{0}=\mathrm{I}_{1}^{(\mathrm{D})} .
$$

\subsubsection{Estado Plano de Tensão -EPT}

Para o caso do EPT a hipótese formulação da consiste em uma aproximação bidimensional de uma estrutura plana fina na coordenada $\boldsymbol{e}_{3}^{r}$ (direção $z$ ), ou seja, $\tau_{33}=0$, na qual os carregamentos externos são aplicados somente nas coordenadas 1 e $2\left(\boldsymbol{e}_{\alpha}^{\mathrm{r}}\right)$ conforme apresentado na figura 3.2 respectivamente. Portanto, aplicando a hipótese

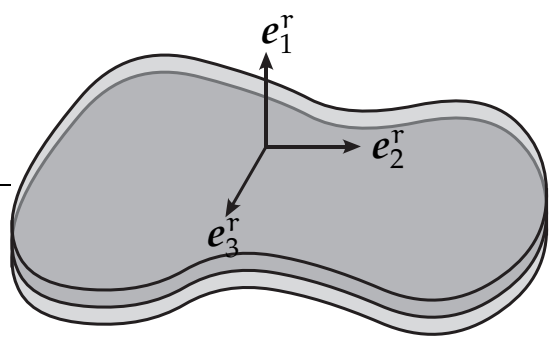

Figura 3.2: Representação da hipótese doEPT $\left(\gamma_{33} \neq 0\right.$ e $\left.\tau_{33}=0\right)$.

EPT no gradiente da transformação (2.2):

$$
\begin{aligned}
\boldsymbol{F}^{(\mathrm{T})} & =\frac{\partial x_{\alpha}}{\partial x_{\beta}^{\mathrm{r}}}\left(\boldsymbol{e}_{\alpha} \otimes \boldsymbol{e}_{\beta}^{\mathrm{r}}\right)+\left(1+\gamma_{33}\right)\left(\boldsymbol{e}_{3} \otimes \boldsymbol{e}_{3}^{\mathrm{r}}\right)=\tilde{\boldsymbol{F}}+\left(1+\gamma_{33}\right)\left(\boldsymbol{e}_{3} \otimes \boldsymbol{e}_{3}^{\mathrm{r}}\right) \\
& =\tilde{\boldsymbol{F}}+\left(\boldsymbol{e}_{3} \otimes \boldsymbol{e}_{3}^{\mathrm{r}}\right)+\gamma_{33}\left(\boldsymbol{e}_{3} \otimes \boldsymbol{e}_{3}^{\mathrm{r}}\right)=\boldsymbol{F}^{\mathrm{D}}+\gamma_{33}\left(\boldsymbol{e}_{3} \otimes \boldsymbol{e}_{3}^{\mathrm{r}}\right),
\end{aligned}
$$

onde $\gamma_{33}^{0} \neq 0$ e $\tau_{33}=0$. Considerando tais condições EPT, o invariante $I_{1}(.2)$ pode ser decomposto da mesma maneira que o gradiente da transformação em (3.16), resultando em:

$$
\mathrm{I}_{1}\left(\boldsymbol{F}^{(\mathrm{T})}\right)=\mathrm{I}_{1}^{(\mathrm{D})}+\tilde{\mathrm{I}}_{1}
$$




\subsubsection{Condição de Axissimetria}

A condição de axissimetria consiste em descrever o movimento de um sólido de revolução no espaço utilizando coordenadas cilíndricas como representado na figura 3.3 resultando em um problema tridimensional que pode ser descrito por duas dimensões Kasper e Taylor (2004):

$$
x_{R}=x_{R}^{r}+u_{R}, \quad x_{\theta}=x_{\theta}^{r}+u_{\theta}, \quad x_{3}=x_{3}^{r}+u_{3},
$$

onde $\chi_{R}, \chi_{\theta}$ e $\chi_{3}$ são referentes ao raio, ângulo e a coordenada axial. Os deslocamentos radiais e axiais são dados por $u_{R}, u_{3}$ e $u_{\theta}$ é referente a torção radial.

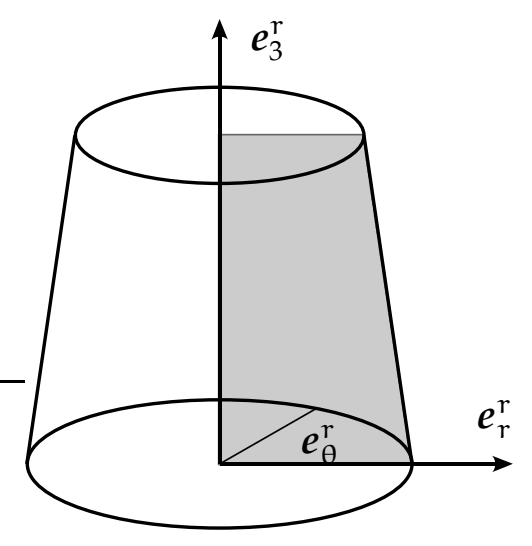

Figura 3.3: Representação da hipótese de axissimetria.

Aplicando a equação 3.18 em 2.2 para obter o gradiente da transformação em relação as coordenadas cilíndricas, temos:

$$
\boldsymbol{F}=\frac{\partial x}{\partial x_{R}^{r}} \otimes \boldsymbol{e}_{\mathrm{R}}^{\mathrm{r}}+\frac{\partial x}{\partial x_{3}^{r}} \otimes \boldsymbol{e}_{3}^{\mathrm{r}}+\frac{1}{\chi_{\mathrm{R}}} \frac{\partial x}{\partial x_{\theta}^{\mathrm{r}}} \otimes \boldsymbol{e}_{\theta}^{\mathrm{r}}
$$

Os vetores de base referentes as coordenadas cilíndrica na configuração de referência $\boldsymbol{e}_{\mathrm{R}}^{\mathrm{r}}, \boldsymbol{e}_{\theta}^{\mathrm{r}}$ dependem dos vetores da base cartesiana $\boldsymbol{e}_{1}^{\mathrm{r}}, \boldsymbol{e}_{2}^{\mathrm{r}}$, as derivadas parciais das bases na coordenada cilíndrica em relação as coordenadas cartesianas são dadas por:

$$
\frac{\partial \boldsymbol{e}_{R}^{r}}{\partial x_{\theta}^{r}}=\boldsymbol{e}_{\theta}^{r}, \quad \frac{\partial \boldsymbol{e}_{\theta}^{r}}{\partial x_{\theta}^{r}}=-\boldsymbol{e}_{\mathrm{R}}^{r}
$$

Aplicando a regra da cadeia para obter as derivadas parciais de $\boldsymbol{e}_{\mathrm{R}}^{\mathrm{r}}$ em relação a $\chi_{r}, \chi_{3}$ 
na equação 3.20 obtém-se:

$$
\frac{\partial e_{R}^{r}}{\partial x_{R}^{r}}=\frac{\partial e_{R}^{r}}{\partial x_{\theta}^{r}} \frac{\partial x_{\theta}^{r}}{\partial x_{R}^{r}}=\frac{\partial x_{\theta}^{r}}{\partial x_{R}^{r}} e_{\theta}^{r}, \quad \frac{\partial e_{R}^{r}}{\partial x_{3}^{r}}=\frac{\partial e_{R}^{r}}{\partial x_{\theta}^{r}} \frac{\partial x_{\theta}^{r}}{\partial x_{3}^{r}}=\frac{\partial x_{\theta}^{r}}{\partial x_{3}^{r}} e_{\theta}^{r}
$$

Portanto aplicando as equações $3.20,3.21 \mathrm{em} 3.19$ chegasse ao gradiente da transformação F em relação as coordenadas cilíndricas:

$$
\begin{aligned}
\boldsymbol{F}= & \frac{\partial x_{R}}{\partial x_{R}^{r}} \boldsymbol{e}_{R} \otimes \boldsymbol{e}_{\mathrm{R}}^{\mathrm{r}}+\frac{\partial x_{R}}{\partial x_{3}^{r}} \boldsymbol{e}_{R} \otimes \boldsymbol{e}_{3}^{\mathrm{r}}+\frac{\partial x_{3}}{\partial x_{R}^{r}} \boldsymbol{e}_{3} \otimes \boldsymbol{e}_{\mathrm{R}}^{\mathrm{r}}+\frac{\partial x_{3}}{\partial x_{3}^{r}} \boldsymbol{e}_{3} \otimes \boldsymbol{e}_{3}^{\mathrm{r}}+ \\
& x_{R} \frac{\partial x_{\theta}}{\partial x_{R}^{r}} \boldsymbol{e}_{\theta} \otimes \boldsymbol{e}_{\mathrm{R}}^{\mathrm{r}}+x_{R} \frac{\partial x_{\theta}}{\partial x_{3}^{r}} \boldsymbol{e}_{\theta} \otimes \boldsymbol{e}_{3}^{r}+\frac{x_{R}}{x_{R}^{r}} \frac{\partial x_{\theta}}{\partial x_{\theta}^{r}} \boldsymbol{e}_{\theta} \otimes \boldsymbol{e}_{\theta}^{r} .
\end{aligned}
$$

\subsubsection{Tensor Tangente Material para Lei Material de neo-Hookiano de Simo-Ciarlet}

Para obter a solução do sistema de equações (3.13) relativas a uma configuração de equilíbrio estático de um sólido, é necessário obter o vetor dos deslocamentos ${ }^{*} \boldsymbol{u}$, ou das variáveis primais que satisfaçam a condição de estacionariedade da energia potencial $\delta \Pi_{\mathfrak{p}}\left(\rho,{ }^{*} \boldsymbol{u}, \delta \boldsymbol{u}\right)=\mathbf{0}$. Para determinar os múltiplos pontos estacionários ${ }^{*} \boldsymbol{u}$, um problema de otimização deve ser solucionado utilizando informações dos gradientes da função $\Pi_{p}$ (2.16), verificando a condição necessária de primeira ordem de Karush-Kuhn-Tucker (ver Seção 3.7).

Para que a condição de estacionariedade seja satisfeita é fundamental obter a linearização do problema tangente $\Delta\left(\delta \Pi_{p}\right)(\underline{3.13})$ que está relacionado com o tensor tangente material ou também chamado de tensor dos módulos tangente material (Pimenta, 2008), sendo obtido pela segunda derivada do funcional de energia de deformação específica $\varphi$ (2.22), que representa a rigidez do sistema quando um ponto material está sujeito a uma deformação ou transformação. É importante que o tensor tangente material satisfaça a condição de objetividade (Holzapfel, 2000; Bertram, 2005) (ver Seção 2.3) e também a condição de elipticidade ou de policonvexidade (ver Seção 2.4).

O tensor tangente material $C_{i ;}^{0}$ referente a lei material $n \mathrm{nH}$ (ver item 2.5) (Campello et al., 2003) é obtido através da derivada parcial do tensor $\tau_{i}^{0}$ PK1 (2.39) em relação ao 
gradiente dos deslocamentos $\gamma_{j}$ :

$$
\frac{\partial \tau_{i}}{\partial \gamma_{j}}=C_{i j}^{0}=\left[\frac{\lambda}{2}\left(1+J^{-1}\right)+\frac{\mu}{J}\right] g_{i} \otimes g_{j}+\left[\frac{\lambda}{2}\left(J-J^{-1}\right)-\frac{\mu}{J}\right] \frac{\partial g_{i}}{\partial f_{j}}+\delta_{i j} \mu I,
$$

onde $i$ e $\boldsymbol{j}$ são referentes as colunas dos seus respectivos tensores $\boldsymbol{P}$ e $\boldsymbol{F}$ conforme demonstrado em (2.2). Considerando a hipótese EPD (3.14) é necessário impor as condição $\gamma_{33}=0$ em $\boldsymbol{F}$, ou seja $f_{33}=1$ para obter o tensor das tensões de engenharia $\boldsymbol{\tau}_{\mathfrak{i}}^{0}(.3)$ correspondente:

$$
\left[\tau_{\alpha}^{0}\right]^{(\mathrm{D})}=\left[\frac{\lambda}{2}\left(J^{2}-1\right)-\mu\right] \frac{g_{\alpha}}{J}+\mu f_{\alpha} .
$$

Utilizando a mesma derivação em (3.23) no tensor das tensões de engenharia PK1 (3.24) é possível obter o tensor tangente material na condiçãoEPD:

$$
\left[\frac{\partial \tau_{\alpha}}{\partial \gamma_{\beta}}\right]^{(D)}=\left[\frac{\lambda}{2}\left(1+J^{-1}\right)+\frac{\mu}{J}\right] g_{\alpha} \otimes g_{\beta}+\left[\frac{\lambda}{2}\left(J-J^{-1}\right)-\frac{\mu}{J}\right] \frac{\partial g_{\alpha}}{\partial f_{\beta}}+\delta_{\alpha \beta} \mu I,
$$

No caso da hipótese EPT (3.16) para obter o tensor tangente material $C_{\mathrm{ij}}^{0}$ matematicamente consistente é necessário impor a condição $f_{33}=1+\gamma_{33}$ no cálculo do tensor das tensões de engenharia $\tau_{i}^{0}$ e no jacobiano do gradiente da transformação $J(\underline{2.8})$, resulta em:

$$
\begin{aligned}
J^{(\mathrm{T})} & =\operatorname{det}\left(\boldsymbol{F}^{(\mathrm{T})}\right)=\left(1+\gamma_{33}\right) \boldsymbol{e}_{3}^{\mathrm{r}} \cdot\left(f_{1} \times f_{2}\right) \\
& =\left(1+\gamma_{33}\right)\left(\boldsymbol{e}_{3}^{\mathrm{r}} \cdot \boldsymbol{g}_{3}\right)=\left(1+\gamma_{33}\right) J^{(\mathrm{D})} \\
& =\left(1+\gamma_{33}\right) g_{33}
\end{aligned}
$$

Também é necessário aplicar a mesma hipótese na equação (2.12), a qual é relativo aos vetores auxiliares de medida de área $g_{\alpha}$ que são obtidos a partir do produto vetorial das componentes vetoriais do gradiente da transformação (2.2). Estes vetores são apresentados por:

$$
g_{1}=f_{2} \times e_{3}^{r}, \quad g_{2}=-f_{1} \times e_{3}^{r}, \quad g_{3}=f_{1} \times f_{2} .
$$

Substituindo (3.26) e (3.27) em (2.39) obtemos o tensor das tensões de engenharia para 
a condição EPT

$$
\left[\boldsymbol{\tau}_{\mathrm{i}}^{0}\right]^{(\mathrm{T})}=\left[\frac{\lambda}{2}\left(J^{2}-1\right)-\mu\right] \frac{\boldsymbol{g}_{\mathrm{i}}}{J}+\mu\left[f_{\alpha}+\left(1+\gamma_{33}\right) \boldsymbol{e}_{3}^{\mathrm{r}}\right]
$$

Isolando o termo $\left[\tau_{33}^{0}\right]$ de $(\underline{3.28)}$ :

$$
\left[\tau_{3}^{0}\right]^{(\mathrm{T})} \cdot e_{3}^{\mathrm{r}}=\left[\tau_{33}^{0}\right]^{(\mathrm{T})}=\left[\frac{\lambda}{2}\left(J^{2}-1\right)-\mu\right] \frac{g_{33}}{J}+\mu\left(1+\gamma_{33}\right) .
$$

Impondo a a condição $\tau_{33}^{0}=$ e isolando o termo $\gamma_{33}$ :

$$
\gamma_{33}=\sqrt{\frac{2 \mu+\lambda}{\lambda J^{2}+2 \mu}}-1
$$

portanto para obter o tensor tangente material é necessário substituir (3.30) na equação (3.25) para obter a componente $f_{3} \cdot \boldsymbol{e}_{3}^{\mathrm{r}}=\left(1+\gamma_{33}\right)$ do gradiente da transformação sob a condição EPT.

\subsection{Métodos de Solução de Sistemas}

Para obter o equilíbrio estático (2.19) apresentado no Capítulo 2.2 que são referentes as EDPs é necessário montar um sistema de equações não lineares (3.13). Nesta seção serão apresentados alguns métodos de solução para problemas não lineares com objetivo de obter os diversos pontos estacionários do sistema (3.13), no caso os vetores referente ao campo dos deslocamentos * $\mathbf{u}$.

\subsubsection{Método de Newton}

Para resolver o sistema de equações não lineares dado pela equação (3.10) e encontrar as suas respectivas raízes "* $\mathbf{u}^{\prime \prime}$, um dos métodos utilizados foi o método de Newton. Para isso, toma-se um vetor com valor inicial das variáveis primais $\left({ }^{(0)} \mathbf{u}\right)$ da função "r $\mathbf{r}(\mathbf{u})$ ", calcula-se a derivada da função em relação a variável e aplica-se o vetor com o 
valor inicial:

$$
\frac{\partial \mathbf{r}((j+1) \mathbf{u})}{\partial \mathbf{u}}=\mathbf{K}_{\mathrm{T}}
$$

subtraindo-se do vetor da solução anterior $\left.{ }_{(j)} \mathbf{u}\right)$ iterativamente " $(j)$ "até que o vetor das raízes estimadas anteriormente seja próximo ao vetor calculado na iteração atual $\left({ }_{j+1} \mathbf{u}\right)$ dentro de uma determinada tolerância " $\epsilon$ ", onde * $\mathbf{u}$ é vetor raiz da função (r) ou o vetor ótimo que minimiza a tolerância " $\epsilon$ ".

Uma das grandes vantagens deste método é a convergência quadrática quando próxima da raiz da função $\delta \Pi_{p}$, reduzindo desta forma o tempo computacional necessário para a solução do sistema de equações não lineares (3.13). A partir da descrição feita é possível escrever a função iterativa do método de Newton que é dada por:

$$
{ }_{(j+1)} \mathbf{u}={ }_{(j)} \mathbf{u}-{ }_{(j)} \Delta \mathbf{u}={ }_{(j)} \mathbf{u}-\left(\mathbf{K}_{\mathrm{T}}\left({ }_{(j)} \mathbf{u}\right)\right)^{-1} \mathbf{r}\left({ }_{(j)} \mathbf{u}\right)
$$

\subsubsection{Método de Newton Incremental}

O método de Newton incremental é aplicado geralmente para a resolver de forma incremental e iterativa sistemas não lineares, cujo ponto estacionário não é possível obter em um único passo via método de Newton (3.32). Utilizando pontos estacionários obtidos em incrementos anteriores, ou seja, pontos mais próximos da nova solução, é possível encontrar um novo ponto estacionário do problema de forma mais rápida e fácil, no entanto, isto não garante obtenção de solução. Para descreve o método de Newton Incremental é necessário incluir um fator de carga proporcional $\bar{\lambda}$ na equação de equilíbrio (3.10), mais especificamente o vetor que se refere aos esforços externos, dado por:

$$
\mathbf{r}\left({ }^{*} \mathbf{u}, \bar{\lambda}\right)=\mathbf{f}_{\mathrm{int}}\left({ }^{*} \mathbf{u}, \bar{\lambda}\right)-\bar{\lambda} \mathbf{f}_{\mathrm{ext}}=\mathbf{0}
$$

onde (i) se refere ao número do incremento de carga. Neste caso o incremento de carga é divido na forma

$$
\bar{\lambda}=\sum_{i=1}^{n_{\text {inc }}}{ }^{(i)} \Delta \bar{\lambda} .
$$


O looping do processo incremental continua até o limite de carga for igual à $\bar{\lambda}$. Podemos resumir o método de Newton incremental a partir do seguinte algoritmo:

Configuração dos valores iniciais de ${ }^{(0)} \mathbf{u}:{ }_{(0)}^{(\mathfrak{i})} \mathbf{u}={ }_{(\mathfrak{j})}^{(\mathfrak{i}-1)} \mathbf{u}$.

Looping Incremental $i=0,1, \ldots$ até ${ }^{(i)} \bar{\lambda} \approx \bar{\lambda}_{\max }$

Looping Iterativo $j=0,1, \ldots$ até tol $\leqslant \epsilon$

1. Cálculo de $\left.\mathbf{r}\left(\begin{array}{c}(\mathfrak{i}) \\ (\mathfrak{j})\end{array}\right){ }^{(i)} \bar{\lambda}\right)$ e $\left.\mathbf{K}_{\mathrm{T}}\left(\begin{array}{l}(\mathfrak{i}) \\ (\mathfrak{j})\end{array}\right){ }^{(i)} \bar{\lambda}\right)$

2. Cálculo do incremento dos deslocamentos: $\mathbf{K}_{\mathrm{T}} \Delta \mathbf{u}=-\mathbf{r}$

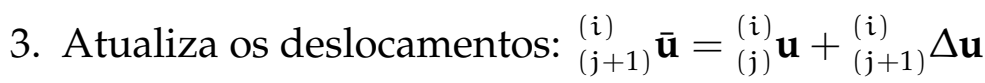

4. Verificação de convergência:

Se $\epsilon \leqslant \epsilon_{\text {lim, }}$ atualiza o vetor dos deslocamentos e o fator de carga proporcional, ${ }_{(0)}^{(i+1)} \mathbf{u}={ }_{(j)}^{(\mathfrak{i})} \overline{\mathbf{u}} ;{ }^{(i+1)} \bar{\lambda}={ }^{(i)} \bar{\lambda}+{ }^{(i)} \Delta \bar{\lambda}$

\subsubsection{Método do Comprimento de Arco - MCA}

Inicialmente o MCA foi proposto por Riks (1979) com objetivo de obter a solução em diversos pontos de instabilidade. No contexto estrutural, busca-se obter a "trajetória de equilíbrio"ou sua respectiva curva de incremento de carga em relação ao vetor dos deslocamentos no ponto de aplicação. Devido ao fato do Método de Newton Incremental não ser capaz de determinar toda a trajetória de equilíbrio da estrutura em configurações instáveis, como, por exemplo, obter os pontos limites, de bifurcação, onde o fator de carga é constante ou sofre reversão e o deslocamento continua aumentando.

A principal justificativa numérica da não convergência do Método de Newton está relacionada as características que a matriz rigidez tangente adquire ao longo do incremento de carga, com o aspecto de positiva semi-definida ou indefinida. Em outra análise o seu determinante se aproxima de zero $\operatorname{det}\left(\mathbf{K}_{\mathrm{T}}\right)=0$. No entanto, é importante diferenciar instabilidade estrutural, da instabilidade material (ver trabalhos de Ball (1976); 
Lahuerta et al. (2013), onde este assunto é abordado).

Ao longo dos anos o MCA vem sendo aprimorado com a utilização da restrição de arco cilíndrico e esférico (Wriggers, 2008; Bonet e Wood, 1997). Sendo a ideia básica do MCA é adicionar uma restrição a mais nas EDPs onde o fator de carga incremental $\bar{\lambda}$ é desconhecido e pode ser determinado utilizando uma restrição de arco:

$$
\mathbf{s}=\left[\begin{array}{c}
\Delta \overline{\mathbf{u}} \\
\overline{\Delta \lambda} \psi
\end{array}\right],
$$

onde s é um vetor que define uma superfície esférica sobre a qual a solução iterativa move-se conforme a convergência do equilíbrio é obtida em uma direção, formando uma trajetória de equilíbrio da estrutura. A constante $\psi$ é referente a um fator de escala que ajusta a dimensão do vetor de carga externa para valores factíveis, com a norma do vetor do campo dos deslocamentos.

O vetor $\Delta \overline{\mathbf{u}}$ define a variação iterativa (i) de $\mathbf{u}$ e $\overline{\Delta \lambda}$ é referente ao fator de variação do incremento de carga. Logo podemos definir estas variáveis incrementais

$$
\left\{\begin{array}{l}
{ }^{(i)} \Delta \mathbf{x}={ }^{(i)} \mathbf{x}-{ }^{(i-1)} \mathbf{x} \\
{ }^{(i)} \Delta \mathbf{p}={ }^{(i)} \overline{\Delta \lambda} \mathbf{f}_{\mathrm{ext}}
\end{array}\right.
$$

e o fator incremento de carga por

$$
\left\{\begin{array}{l}
{ }^{(i)} \bar{\Delta} \lambda={ }^{(i)} \bar{\lambda}-{ }^{(i-1)} \bar{\lambda} \\
{ }^{(i)} \bar{\Delta} \bar{\lambda}=\bar{\gamma}+{ }^{(i-1)} \bar{\Delta} \bar{\lambda}
\end{array},\right.
$$

onde $\bar{\Delta} \bar{\lambda}$ se refere a variação do incremento de carga e $\bar{\gamma}$ a variação iterativa (j) de $\bar{\lambda}$.

Portanto o comprimento de arco ou o seu respectivo raio s pode ser calculado à partir do produto interno do vetor ${ }^{(i)} \mathbf{s}($ (3.36):

$$
{ }^{(i)} \Delta \mathbf{x} \cdot{ }^{(i)} \Delta \mathbf{x}+\psi^{2} \overline{\Delta \lambda^{2}}={ }^{(i)} \Delta s^{2} .
$$


Levando em conta que o fator de incremento de carga $\bar{\lambda}$ é alterado durante o processo iterativo para satisfazer a restrição ${ }^{(i)}$ s na equação (3.36), portanto, é necessário reescrever o equilíbrio (3.33) incluindo $\bar{\gamma}$ :

$$
\mathbf{r}\left(\mathbf{u}^{(i)} \bar{\lambda}\right)+\mathbf{K}_{\mathrm{T}}\left(\mathbf{u}^{(i)} \bar{\lambda}\right) \Delta \mathbf{u}-\bar{\gamma}^{(i)} \mathbf{f}_{\mathrm{ext}}=\mathbf{0}
$$

Para resolver as equações acima apresentadas, é necessário obter o vetor dos deslocamento incrementais $\Delta \mathbf{u}$, no entanto é fundamental dividi-lo em relação ao vetor do resíduo e da força externa do seguinte modo:

$$
\left\{\begin{aligned}
\Delta \mathbf{u} & =\Delta \mathbf{u}_{\mathrm{r}}+\bar{\gamma} \Delta \mathbf{u}_{0} \\
\tilde{\mathbf{u}} & ={ }^{(i-1)} \mathbf{u}+\Delta \mathbf{u} \\
\mathbf{u}_{\mathrm{r}} & =\mathbf{K}_{\mathrm{T}}(\tilde{\mathbf{u}})^{-1} \mathbf{r}\left(\tilde{\mathbf{u}},{ }^{(i)} \bar{\lambda}\right) \\
\mathbf{u}_{0} & =-\mathbf{K}_{\mathrm{T}}(\tilde{\mathbf{u}})^{-1} \mathbf{f}_{\mathrm{ext}}
\end{aligned}\right.
$$

Expandindo a equação (3.38) obtemos uma equação de segundo grau em relação a variação do iterativa do incremento de carga $\bar{\gamma}$ :

$$
{ }^{(i)} \Delta \overline{\mathbf{u}} \cdot{ }^{(i)} \Delta \overline{\mathbf{u}}+\left({ }^{(i)} \overline{\Delta \lambda}+\bar{\gamma}\right)^{2} \psi^{2}={ }^{(i)} \Delta s^{2} \text {, }
$$

isolando as demais constantes em relação a ordem do poliônimo, obtemos:

$$
a_{1} \bar{\gamma}^{2}+a_{2} \bar{\gamma}+a_{3}=0
$$

onde as constantes $a_{i}$ são dadas por:

$$
a_{i}=\left\{\begin{array}{l}
a_{1}=\Delta \mathbf{u}_{0} \cdot \Delta \mathbf{u}_{0}+\psi^{2} \overline{\Delta \lambda^{2}} \\
a_{2}=2 \Delta \mathbf{u}_{0} \cdot\left({ }^{(i)} \mathbf{u}+\mathbf{u}_{r}\right)+2\left({ }^{(i)} \overline{\Delta \lambda} \psi^{2}\right) \\
a_{3}=\Delta \mathbf{u}_{r} \cdot\left(2^{(i)} \mathbf{u}+\mathbf{u}_{r}\right)+{ }^{(i)} \mathbf{u} \cdot{ }^{(i)} \mathbf{u}-s_{i}^{2}
\end{array}\right.
$$

Resolvendo (3.44), obtemos duas raízes referente a variação do iterativa do incremento de carga $\bar{\gamma}^{(1)}$ e $\bar{\gamma}^{(2)}$, no entanto a variação correta é aquela, que minimiza o ângulo entre 
cada incremento de arco $\theta$. O cálculo do ângulo $\theta$ é dada por:

$$
\cos \left({ }^{(i)} \theta\right)=\frac{{ }^{(i)} \mathbf{u} \cdot{ }^{(i+1)} \mathbf{u}}{s^{2}}
$$

\subsubsection{Método do Controle Generalizado dos Deslocamentos -MCGD}

O MCGD foi proposto por Yang e Shieh (1990), é um algoritmo capaz que solucionar diversos pontos de instabilidade de uma estrutura e obter seu caminho instável (pathfollow). É um método incremental (i) e iterativo (j) que consiste em um juste automático do passo incremental $(\bar{\Delta} \lambda)$ utilizando o $\overline{P G R}$ da estrutura. Pode se generalizar como um Método do Comprimento de Arco Ortogonal (Cardoso e Fonseca, 2007; Wriggers, 2008; Bonet e Wood, 1997). O método relaciona os deslocamentos incrementais em dois passos sucessivos para avaliar melhor o comportamento da estrutura ou estabilidade do sistema, através da seguinte expressão para o cálculo do fator de carga proporcional:

$$
{ }_{(\mathrm{j})}^{(\mathfrak{i})} \overline{\Delta \lambda}=-\frac{{ }_{(0)}^{(0)} \mathbf{u}_{0} \cdot{ }_{(0)}^{(0)} \Delta \mathbf{u}_{\mathrm{r}}}{{ }_{(0)}^{(\mathrm{i}-1)} \mathbf{u}_{0} \cdot{ }_{(0)}^{(\mathrm{i})} \Delta \mathbf{u}_{0}},
$$

onde os índices do lado esquerdo se referem ao incremento de carga $(\mathfrak{i})$ e iteração $(\mathfrak{j})$. $\mathrm{O}$ fator de carga proporcional $\overline{\Delta \lambda}$ é composto pelo vetor dos deslocamentos lineares $\mathbf{u}_{0}$ que é obtido resolvendo o sistema linear $\mathbf{K}_{\mathrm{T}}^{0} \backslash \mathbf{f}_{\mathrm{ext}}$ considerando os carregamentos externos totais e o vetor dos deslocamentos residuais atualizados representado por $\mathbf{u}_{\mathrm{r}}$.

Para toda primeira iteração $(j=0)$ do método, é necessário calcular o $\underline{\mathrm{PGR}}$, dado por:

$$
P G R=\frac{{ }_{(0)}^{(0)} \mathbf{u}_{0} \cdot{ }_{(\mathfrak{j})}^{(\mathfrak{i})} \mathbf{u}_{\mathrm{r}}}{{ }_{(\mathrm{i}-1)}^{(0)} \mathbf{u}_{0} \cdot{ }_{(\mathfrak{j})}^{(\mathfrak{i})} \mathbf{u}_{0}},
$$

de modo que o incremento no fator de carga proporcional inicial leve em consideração a direção do carregamento, pois, o carregamento pode reverter se $P G R<0$, portanto:

$$
\text { (i) } \overline{\Delta \lambda}=\operatorname{sign}(P G R){ }_{0}^{0} \overline{\Delta \lambda} \sqrt{|P G R|} \text {, }
$$




$$
\underset{(\mathfrak{j}+1)}{(\mathfrak{i}+1)} \overline{\Delta \lambda}=\underset{(\mathfrak{j})}{(\mathfrak{i})} \bar{\lambda}+\underset{(\mathfrak{j})}{(\mathfrak{i})} \overline{\Delta \lambda}
$$

O PGR possui duas propriedades importantes: a primeira delas é o numerador e o denominador de sua expressão que representam respectivamente, os deslocamentos no primeiro passo e os deslocamentos no passo corrente. Desta forma, o $\overline{P G R}$ representa a variação da rigidez da estrutura em relação ao incremento inicial e consequentemente pode ser utilizado para determinar de forma automática o ajuste do tamanho do passo ou fator de carga proporcional $(\bar{\Delta} \bar{\lambda})$. A segunda característica é o sinal, quando negativo, a curva de carga se próxima de pontos instáveis, pois, seu sinal depende do produto escalar entre ${ }_{(0)}^{(i-1)} \mathbf{u}_{0} \mathrm{e}_{(0)}^{(\mathfrak{i})} \mathbf{u}_{0}$ fornecendo a indicação da mudança no sinal do incremento de carga ou reversão do carregamento. Portanto, comparando o MCGD ao MCA a única diferença na implementação se refere a estimativa de $\bar{\gamma}$, que é feita através do PGR utilizando as equações (3.46), (3.47) e (3.48) respectivamente, ou seja, ${ }_{(0)}^{(i)} \bar{\gamma}={ }_{(0)}^{(i)} \overline{\Delta \lambda}$.

\subsection{Instabilidade Elástica}

Estruturas reais respondem a carregamento externo muitas vezes de forma não linear, ou seja, a relação entre os carregamentos externo e os deslocamentos são não linear. Geralmente estas não linearidades estão associadas a mudanças súbitas do comportamento do estrutural, tais mudanças súbitas podem ser classificadas como um tipo de instabilidade. Uma das causas associadas a este comportamento instável está ligada ao mecanismo de flambagem, que geralmente é caracterizado por uma falha estrutural súbita associada a múltiplas soluções de equilíbrio a um certo fator de carregamento (Wriggers, 2008; Marsden e Hughes, 1994; Bažant e Cedolin, 2010). Ambos os casos só podem ocorrer em sistemas não lineares e portanto, necessitam serem tratados com base em um modelo teórico adequado.

Todos os sistemas conservativos não lineares sujeitos a carregamentos externos para os quais exista um funcional $\delta \Pi_{p}$ apresentam algum tipo de instabilidade ou configuração de equilíbrio crítica (flambagem, divergência), isto porque de acordo com o teorema de Lagrange-Dirichlet um sistema que descreve a configuração de equilíbrio de um sólido 
seja estável é necessário que a segunda variação da energia potencial $\delta^{2} \Pi_{p}$ seja positivodefinida nesta configuração. No entanto, esta é uma condição suficiente e não uma condição necessária segundo o teorema de Lyapunov que é muito mais rigoroso na análise de estabilidade. Portanto, quando a energia potencial $\delta^{2} \Pi_{p}$ não é positivo-definida o sistema pode ou não ser estável. A ausência do mínimo local de $\delta^{2} \Pi_{p}$ (ausência da definição de positivo-definida) não implica necessariamente em instabilidade pelo primeiro teorema de Lyapunov, logo pode-se dizer que o teorema de Lagrange-Dirichlet é um caso particular do primeiro teorema de Lyapunov (Leipholz, 2013; Lyapunov, 1992).

Para uma análise da trajetória de equilíbrio baseado no teorema da energia potencial é necessário analisar a segunda variação de $\delta^{2} \Pi_{p}$, portanto, a partir da primeira variação da energia potencial (2.19) é possível obter $\delta^{2} \Pi_{p}$ :

$$
\begin{aligned}
\delta^{2} \Pi_{p}(\rho, \boldsymbol{u}, \delta \boldsymbol{u}) & =\int_{\Omega^{\mathrm{r}}}\left(\frac{\partial^{2} \varphi}{\partial \boldsymbol{F}^{2}} \delta \boldsymbol{F}\right): \delta \boldsymbol{F} d v^{\mathrm{r}}+\int_{\partial \Omega^{r}}\left(\frac{\partial^{2} \varphi_{\mathrm{t}}}{\partial \boldsymbol{u}^{2}} \delta \boldsymbol{u}\right) \cdot \delta \boldsymbol{u} d a^{\mathrm{r}}+ \\
& +\int_{\Omega^{\mathrm{r}}}\left(\frac{\partial^{2} \varphi_{\mathrm{b}}}{\partial \boldsymbol{u}^{2}} \delta \boldsymbol{u}\right) \cdot \delta \boldsymbol{u} d v^{\mathrm{r}}
\end{aligned}
$$

A linearização de $\delta \Pi_{p}$ (3.13) é importante para obter o sistema tangente do problema, pois, representam as equações de estado da deformação elástica, cujo processo é reversível e preserva o equilíbrio termodinâmico. Portanto, a matriz rigidez tangente $\mathbf{K}_{\mathrm{T}}(\underline{3.13})$ representa a aproximação da Hessiana do funcional $\Pi_{p}$ em relação vetor do campo dos deslocamentos ${ }^{*} \boldsymbol{u}$. Neste caso $\mathbf{K}_{\mathrm{T}}$ é uma matriz simétrica, pois como dito, o sistema $\Pi_{p}$ é conservativo e ${ }^{*} \boldsymbol{u}$ independe do fator de carregamento $\bar{\lambda}$. No entanto, para uma análise mais precisa da estabilidade do problema, pode ser necessário incluir efeitos de segunda ordem, como pre-tensão e imperfeições do problema aproximado (Leipholz, 2013).

A verificação da estabilidade do equilíbrio de um sólido pode ser feita utilizando a matriz do sistema tangente, se $\mathbf{K}_{\mathrm{T}}$ é positiva-definida, $\log 0 \delta^{2} \Pi_{\mathrm{p}}$ também é positivadefinida, portanto, a condição suficiente de estabilidade é satisfeita. Como $\mathbf{K}_{\mathrm{T}}=\mathbf{K}_{\mathrm{T}}^{\mathrm{T}}$ $\operatorname{logo}$ se $\operatorname{det} \mathbf{K}_{\mathrm{T}}>0$ a condição suficiente de estabilidade também será satisfeita. Um 
exame mais detalhado da estabilidade do sistema tangente $\mathbf{K}_{\mathrm{T}}$ (positiva-definida) pode ser feito pela análise dos seus respectivos autovalores $\kappa_{i}$ dado por:

$$
\left(\mathbf{K}_{\mathrm{T}}\left({ }^{*} \boldsymbol{u}\right)-\mathrm{K}_{(i)} \mathbf{I}\right) \boldsymbol{\Phi}_{(\mathfrak{i})}=\mathbf{0}
$$

onde $\boldsymbol{\phi}_{(i)}$ é referente autovetor da solução $(i)$, ou seja, $\kappa_{(1)} \leqslant \kappa_{(2)} \leqslant \ldots \leqslant \kappa_{(m)}$ e $m$ é referente a dimensão de $\mathbf{K}_{\mathrm{T}} \in \mathbb{R}^{\mathrm{m} \times \mathrm{m}}$.

Por consequência, quando $\kappa_{(1)} \approx 0$ a estrutura estará em uma condição próxima de uma configuração de equilíbrio crítica em relação ao incremento de carga ${ }^{(i)} \bar{\lambda}, \operatorname{logo}$ o vetor do campo dos deslocamentos ${ }^{(1)} \mathbf{u}$ será ortogonal a ${ }^{(1)} \mathbf{K}_{\mathrm{T}}$, pois, ${ }^{(1)} \mathbf{u}^{\mathrm{T}(1)} \mathbf{K}_{\mathrm{T}}{ }^{(1)} \mathbf{u}^{\mathrm{T}} \approx 0$. Portanto, o vetor dos deslocamentos e dos autovetor (1) são referentes ao modo de flambagem da estrutura, assim sendo temos a seguinte relação:

$$
\frac{{ }^{(1)} \Delta \mathbf{u}}{\left\|{ }^{(1)} \Delta \mathbf{u}\right\|} \approx \frac{\boldsymbol{\phi}_{(1)}}{\left\|\boldsymbol{\phi}_{(1)}\right\|}
$$

Outro método muito eficiente utilizado para a verificação de estabilidade da trajetória de equilíbrio é o PGR dado em (3.46), quanto mais próximo o PGR de zero, mais próximo de um ponto instável, no entanto, não é possível classificar o tipo de instabilidade. Sendo este um parâmetro utilizado no MCGD para aumentar ou reduzir o fator de carga proporcional e também reverter o carregamento quando a estrutura se encontra em um ponto instável evitando desta forma resolver uma equação de segundo grau (ver seções 3.5.3 e 3.5.4). No entanto, fazendo uma análise do produto interno dos autovetores em relação ao vetor do carregamento externo, podemos classifica-los em relação ao seu valor:

1. Ponto Limite, neste caso ${ }^{(i)} \Delta \bar{\lambda}$ se aproxima de zero e a variação dos deslocamentos ${ }^{(i)} \Delta \overline{\mathbf{u}}$ se aproxima do modo do autovetor $\boldsymbol{\phi}_{(i)}$, representa um máximo da o incremento de carga ${ }^{(i)} \bar{\lambda}$.

$$
\phi_{(i)} \cdot \mathbf{f}_{\text {ext }} \neq 0
$$

2. Ponto de Bifurcação das trajetórias de equilíbrio, podendo ser classificada como 
simétrica ou assimétrica dependendo o valor de ${ }^{(i)} \Delta \bar{\lambda}$. Se ${ }^{(i)} \Delta \bar{\lambda}=0$ então a bifurcação é do tipo simétrica e $\boldsymbol{\phi}_{(\mathfrak{i})}$ se aproxima do modo do vetor ${ }^{(i)} \Delta \overline{\mathbf{u}}$. Caso contrário a bifurcação é do tipo assimétrica e o modo do autovetor $\boldsymbol{\phi}_{(\mathfrak{i})}$ não tem correlação com o modo do vetor ${ }^{(i)} \Delta \overline{\mathbf{u}}$

$$
\phi_{(i)} \cdot \mathbf{f}_{\mathrm{ext}}=0 \text {. }
$$

Considerando que o problema não-linear dado pela equação de equilíbrio (3.9) admita múltiplas soluções (problema não convexo), mantendo elipticidade da lei material (ver item 2.4), consideramos que o mecanismo de instabilidade estrutural está relacionado com flambagem elástica da estrutura. Existem outros mecanismos de instabilidade, no entanto, não são o foco desta tese, para um entendimento mais aprofundado sobre a teoria de estabilidade estrutural ver os trabalhos de Marsden e Hughes (1994); Bažant e Cedolin (2010).

A flambagem pode ser analisada antes ou próxima da configuração crítica, na préflambagem e após a configuração crítica ou na pós-flambagem. Na figura 3.4 é apresentado o gráfico das configuracões de equilíbrio crítica do modelo de pórtico proposto Lee e Manuel (1968); Schweizerhof e Wriggers (1986). É uma estrutura muito utilizada como referência na literatura para a avaliação de diversos métodos de solução não lineares (ver Seções 3.5.1, 3.5.4), pois, este modelo possui solução analítica e exibe muitas configurações críticas $A, B, C, D, E, F G$, sendo o efeito de estalo ou "snap-back"o mais instável de todos e indicado pelo ponto C (Bažant e Cedolin, 2010).

A utilização do mecanismo de flambagem tem se mostrado promissor como uma ferramenta de grande potencial no projeto de estruturas e dispositivos inteligentes como MEMS, atuadores, sistemas de coleta de energia com o objetivo de maximizar o seu desempenho. Uma revisão mais detalhada é feita por Hu et al. (2015), onde uma extensiva revisão sobre aplicações, estágio atual e futuras aplicações sobre o tema em questão.

Na figura 3.5 são apresentados dois gráficos com comportamento do modelo do 


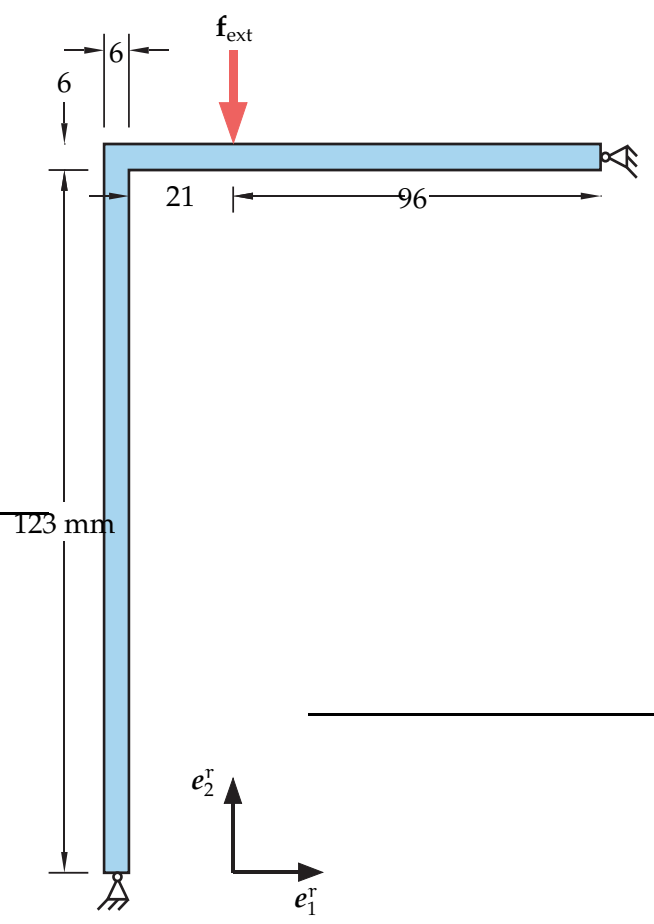

(a)
Gráfico de Flambagem

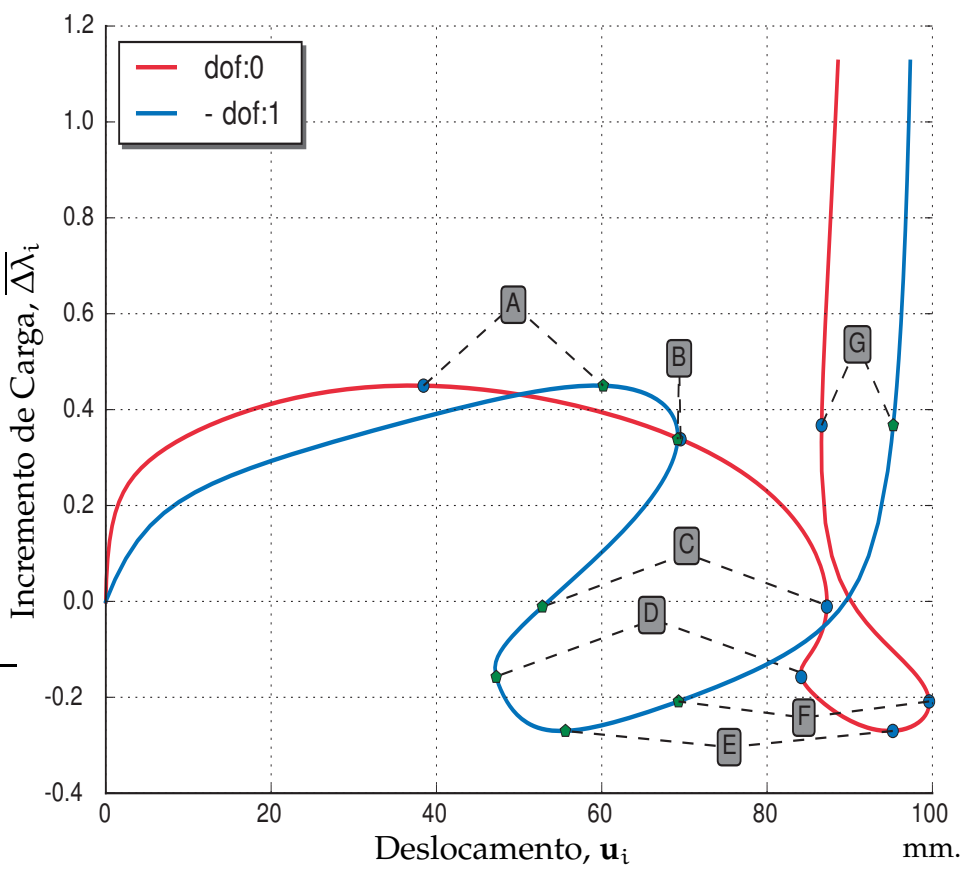

(b)

Figura 3.4: (a) Configuração do modelo do pórtico Lee. (b) Curva incremental de carga versus deslocamento da estrutura nos nós de aplicação.

pórtico Lee ao longo de diversas configurações de equilíbrio crítica (A, B, C, D, E, F e G), onde é possível visualizar a variação da energia potencial $\Pi_{p}$ em diversas configurações críticas da estrutura e na sua conversão em energia cinética. No caso, a energia cinética, é a energia que será utilizada nas portas de saída do mecanismo flextensional para atuação.
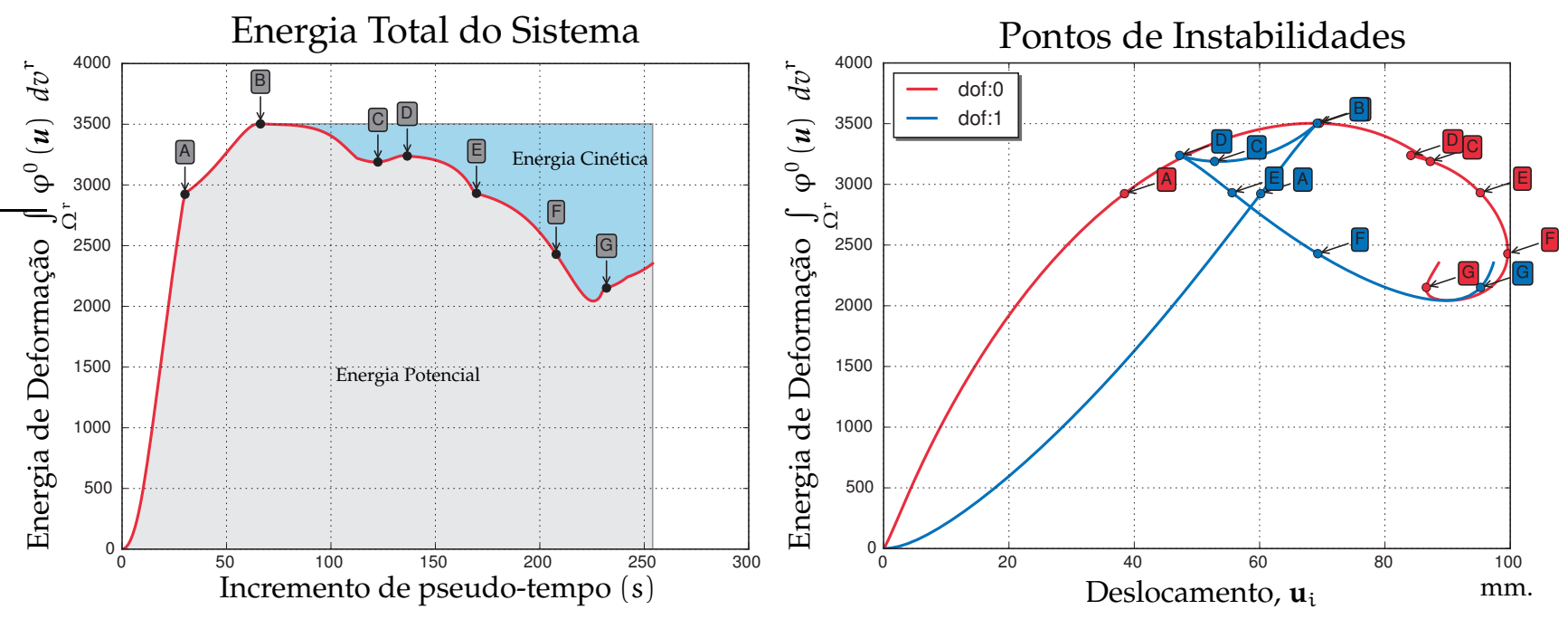

Figura 3.5: Gráfico da Energia de Deformação Específica $\left(\varphi^{0}\right)$ em relação ao deslocamento e ao incremento de pseudo-tempo do modelo do pórtico Lee. 


\subsection{Otimização Numérica}

Para utilizar uma ferramenta numérica de otimização é preciso identificar a função objetivo, restrições e variáveis de projeto. Nosso objetivo é encontrar a ótima distribuição para as variáveis de projeto que minimizem ou maximizem a função objetivo e que satisfaçam as restrições de projeto no MOT.

A formulação da função objetivo $f$, restrições $h_{j}, g_{e}$ e variáveis de projeto $\rho_{i}$ para um determinado problema é conhecido como modelagem, onde uma correta formulação é o primeiro passo e o mais importante no processo de otimização. Uma vez que o modelo tenha sido formulado, um algoritmo ou método de otimização ou um conjunto de métodos podem ser selecionados para o MOT, desta forma busca-se iterativamente a solução ótima ou o respectivo ponto estacionário do problema * $(\bullet)$.

De forma generalista problema de otimização pode ser apresentado por:

$$
\begin{array}{ll}
\underset{\rho_{i} \in \mathbb{R}^{n}}{\operatorname{Minimizar}} & f\left(\rho_{i}\right) \\
\text { Sujeito à: } & h_{j}\left(\rho_{i}\right)-b_{j}=0 \\
& g_{e}\left(\rho_{i}\right)-b_{e} \leqslant 0 \\
& \underline{\rho} \leqslant \rho_{i} \leqslant \bar{\rho}
\end{array}
$$

onde $b_{j}$ e $b_{e}$ são refere ao valores das restrições de igualdade e desigualdade e $\underline{\rho}$ e $\bar{\rho}$ são referentes as restrições de caixa das variáveis de projeto $\underline{\rho} \leqslant \rho_{i} \leqslant \bar{\rho} \in \mathbb{R}^{n}$. Logo o conjunto viável do problema é definido por $F=\left\{\rho_{i} \in \mathbb{R}^{n} \mid h_{j}\left(\rho_{i}\right)-b_{j}=0\right.$ e $\left.g_{e}\left(\rho_{i}\right)-b_{e} \leqslant 0\right\}$.

Esta generalização do problema (3.54) contém restrições de igualdade (j) e de desigualdade (e) que não devem ser violadas e para tal, podemos rescrever o problema de otimização em uma função Lagrangiana (Nocedal e Wright, 2006; Byrd et al., 1999) sem restrições da seguinte forma:

$$
\mathcal{L}\left(\rho_{i}, \Lambda_{j}, \Gamma_{e}\right)=f\left(\rho_{i}\right)+\Lambda_{j}\left(h_{j}\left(\rho_{i}\right)-b_{j}\right)+\Gamma_{e}\left(g_{e}\left(\rho_{i}\right)-b_{e}\right)
$$


onde os parâmetros $\Lambda_{j}, \Gamma_{e} \in \mathbb{R}^{+}$são referentes aos multiplicadores de Lagrange ou variáveis duais do problema, que devolvem de forma indireta as restrições através da solução $\nabla \mathcal{L}\left({ }^{*} \rho_{i},{ }^{*} \Lambda_{j},{ }^{*} \Gamma_{e}\right)=[0,0,0]$, obtendo a condição de condição de estacionariedade:

$$
\begin{gathered}
\nabla \mathcal{L}\left({ }^{*} \rho_{i},{ }^{*} \Lambda_{j},{ }^{*} \Gamma_{e}\right)=\left[\frac{\partial \mathcal{L}}{\partial \rho_{k}}, \frac{\partial \mathcal{L}}{\partial \Lambda_{l}}, \frac{\partial \mathcal{L}}{\partial \Gamma_{y}}\right]=[0,0,0], \\
\frac{\partial \mathcal{L}}{\partial \rho_{i}}=\frac{\partial f}{\partial \rho_{k}}+\Lambda_{j} \frac{\partial h_{j}}{\partial \rho_{k}}+\Gamma_{e} \frac{\partial g_{e}}{\partial \rho_{k}}=0, \\
\frac{\partial \mathcal{L}}{\partial \Lambda_{l}}=\delta_{l j}\left(h_{j}\left(\rho_{i}\right)-b_{j}\right)=0, \\
\frac{\partial \mathcal{L}}{\partial \Gamma_{y}}=\delta_{e y}\left(h_{e}\left(\rho_{i}\right)-b_{e}\right)=0,
\end{gathered}
$$

e com a condição de complementaridade $\Lambda_{j} h_{j}\left(\rho_{i}\right)=0$ sejam satisfeitas.

A condição de estacionariedade implica que os multiplicadores de Lagrange $\left(\Lambda_{j} \geqslant 0\right)$ sejam estritamente positivos apenas quando as restrições $\left(h_{j}\right)$ estiverem ativas, caso contrário iguala-se a zero quando não ativas $\left(\Lambda_{j}=0\right)$. Portanto, se as condições de estacionariedade e complementaridade forem satisfeitas então pode-se afirmar que * $\rho_{i}$ minimiza a função $f$, pois $f\left(\rho_{i}\right) \geqslant f\left({ }^{*} \rho_{i}\right)$, logo são condições necessárias e suficientes do problema. No entanto, não é possível qualificar se * $\rho_{i}$ é um mínimo local ou global de $f$, tais também são referidas como condições necessárias e suficientes de primeira ordem.

\subsection{Método de Ponto Interior}

O método de ponto interior ou também chamado de método da barreira logarítmica é um método de programação matemática inicialmente proposto por John von Neumann (Dantzig e Thapa, 2006) que consiste em substituir as restrições de desigualdade $((\bullet) \leqslant 0)$ por uma função logarítmica de barreira que mantém a busca da solução ótima dentro do domínio viável de projeto. A função de barreira é dada por:

$$
B\left(\rho_{i}, \mu_{\mathrm{o}}\right)=-\mu_{\mathrm{o}} \ln s_{e},
$$


onde $\mu_{\mathrm{o}}>0$ é denominado de parâmetro de barreira e $s_{e} \in \mathbb{R}^{+}$são as variáveis de folga. O parâmetro de barreira define a aproximação da função de restrição de desigualdade, conforme a função objetivo $f$ se aproxima do valor ótimo, $\mu_{\mathrm{o}} \rightarrow 0$.

Considerando o problema de otimização (3.54), a função Lagrangiana (3.55) com a função de barreira logarítmica pode ser apresentada da seguinte forma:

$$
\mathcal{L}\left(\rho_{i}, s_{e}, \Lambda_{j}, \Gamma_{e}\right)=f\left(\rho_{i}\right)-\mu_{o} \ln s_{e}+\Lambda_{j}\left(h_{j}\left(\rho_{i}\right)-b_{j}\right)+\Gamma_{e}\left(g_{e}\left(\rho_{i}\right)-b_{e}+s_{e}\right)
$$

Com a finalidade de obter a condição de estacionariedade de (3.58) é necessário obter as derivadas parciais de primeira ordem $\nabla \mathcal{L}\left({ }^{*} \rho_{i},{ }^{*} s_{e},{ }^{*} \Lambda_{j},{ }^{*} \Gamma_{e}\right)=[0,0,0,0]$ :

$$
\begin{aligned}
& \nabla \mathcal{L}\left({ }^{*} \rho_{i},{ }^{*} s_{e},{ }^{*} \Lambda_{j},{ }^{*} \Gamma_{e}\right)=[0,0,0,0] \\
& \frac{\partial \mathcal{L}}{\partial \rho_{i}}=\frac{\partial f}{\partial \rho_{k}}+\Lambda_{j} \frac{\partial h_{j}}{\partial \rho_{k}}+\Gamma_{e} \frac{\partial g_{e}}{\partial \rho_{k}}=0 \\
& \frac{\partial \mathcal{L}}{\partial s_{y}}=-\delta_{e y}\left(\mu_{\mathrm{o}} \frac{1}{s_{e}}-\Gamma_{e}\right)=0 \\
& \frac{\partial \mathcal{L}}{\partial \Lambda_{l}}=\delta_{l j}\left(h_{j}\left(\rho_{i}\right)-b_{j}\right)=0 \\
& \frac{\partial \mathcal{L}}{\partial \Gamma_{y}}=\delta_{e y}\left(h_{e}\left(\rho_{i}\right)-b_{e}\right)=0
\end{aligned}
$$

A condição de complementaridade $\Lambda_{j} h_{j}\left(\rho_{i}\right)=0$ impõem aos multiplicadores de Lagrange $\left(\Lambda_{j} \geqslant 0\right)$ o valor zero se as restrições estiverem inativas $\left(\Lambda_{j}=0\right)$.

\subsection{Método dos Mínimos Quadrados}

$\mathrm{O}$ MMQ é uma recurso utilizado em otimização para encontrar o melhor ajuste para um conjunto de dados ou funções, minimizando a somatória dos quadrados das diferenças entre o valor estimado e os valores observados. Em outras palavras um processo de minimização iterativo do erro quadrático das funções a serem estimadas.

Neste trabalho o MMQ foi utilizado para obter uma solução aproximada de um 
problema mal condicionado, onde as restrições de projeto se encontram fora do conjunto viável do problema $F$ (3.54). Neste caso impondo de forma adaptativa as restrições de modos específicos de flambagem, relaxando de forma gradual as funções de restrições que são impostas de forma indireta no problema de otimização.

A função MMQ é dada por uma somatória de " $v^{\prime \prime}$ funções de restrições:

$$
\sum_{n=1}^{v} S_{a}\left(\rho_{i}\right)=\sum_{a=1}^{v} \frac{1}{2}\left(\frac{\vartheta_{a}\left(\rho_{i}\right)-k_{a}}{w_{a}}\right)^{2},
$$

onde $k_{a}$ é referente ao valor da restrição e $w_{a}$ o peso da função que é alterado de forma iterativa conforme o valor da função de erro $S_{a}$ é minimizada.

Inicialmente o valor do peso da função $S_{a}$ é dado por $w_{a}=1$ e ao longo do processo de otimização conforme o valor do erro se reduz, o peso é alterado iterativamente subtraindo-se 0,02 até o valor final de $w_{a}=0,01$, desta forma se atribui $\vartheta_{a} \sim k_{a}$ de forma gradativa.

A particularidade da modificação do MMQ como apresentado em (3.60) em estabelecer um peso $w_{a}$ está no fato de favorecer quadraticamente as derivadas da função de erro $S_{\mathrm{a}}$ (3.61), já que o parâmetro de peso $w_{\mathrm{a}} \leqslant 1 \in \mathbb{R}^{+}$.

$$
\frac{\partial S_{a}\left(\rho_{i}\right)}{\partial \rho_{j}}=\left(\frac{\vartheta_{a}\left(\rho_{i}\right)-k_{a}}{w_{a}^{2}}\right) \frac{\partial \vartheta_{a}}{\partial \rho_{j}}
$$

Logo a forma geral do problema de otimização (3.54) com as restrições imposta via MMQ com as restrições de igualdade e desigualdade é dada por:

$$
\begin{array}{ll}
\underset{\rho_{i} \in R^{\mathfrak{n}}}{\operatorname{Minimizar}} & f\left(\rho_{i}\right)+S_{a}\left(\rho_{i}\right) \\
\text { Sujeito à: } & h_{j}\left(\rho_{i}\right)-b_{j}=0 \\
& g_{e}\left(\rho_{i}\right)-b_{e} \leqslant 0 \\
& \underline{\rho} \leqslant \rho_{i} \leqslant \bar{\rho}
\end{array}
$$

Esta forma geral do problema (3.62) também pode ser expressa pela com a somatória de 
(3.61) na função Lagrangiana (3.56):

$$
\mathcal{L}\left(\rho_{i}, \Lambda_{j}, \Gamma_{e}\right)=f\left(\rho_{i}\right)+\frac{1}{2}\left(\frac{\vartheta_{a}\left(\rho_{i}\right)-k_{a}}{w_{a}}\right)^{2}+\Lambda_{j}\left(h_{j}\left(\rho_{i}\right)-b_{j}\right)+\Gamma_{e}\left(g_{e}\left(\rho_{i}\right)-b_{e}\right)
$$




\section{Capítulo 4}

\section{Método de Otimização Topológica}

Essencialmente, o MOT procura distribuir material no interior de um domínio fixo de projeto, discretizado em elementos finitos, que permanecerá fixo durante o processo de otimização (Bendsøe e Kikuchi, 1988; Bendsoe e Sigmund, 2013). Um conceito importante deste método está relacionado na distribuição das propriedades físicas de um dado material entre as restrições de caixa dentro domínio fixo pois a distribuição de material em somente dois parâmetros é mal-posto, ou seja, não apresenta solução única, necessitando de uma relaxação das variáveis de restrições de complexidade, tais como restrição de perímetro ou de complexidade (Haber et al., 1996; Bourdin, 2001).

A relaxação das variáveis de projeto através de um modelo de material permite uma gradação entre os limites inferiores $\underline{\rho}$ e superiores $\bar{\rho}$ da variável de projeto, ou seja o material pode assumir valores intermediários entre as restrições de caixa do problema tornando o processo de otimização bem posto. Assim, as variáveis de projeto se tornam as funções que definem uma mistura de diversos materiais em cada ponto material $x^{r}$ do domínio de projeto $\Omega^{r} \subset \mathbb{R}^{n}$.

Os principais modelos de materiais (Seção 4.1) que definem uma mistura em microescala de dois ou mais materiais são o métodos da homogeneização (Suzuki e Kikuchi, 1991) e das densidades, ente eles o SIMP (Bendsøe e Sigmund, 1999). Baseado em todas estas considerações podemos definir o problema genérico de OTcomo um problema de 
programação matemática de otimização a ser minimizado

$$
\begin{array}{ll}
\underset{\left.\left.\rho\left(\boldsymbol{x}^{\mathrm{r}}\right) \in\right] 0,1\right] \in \mathbb{R}^{+}}{\operatorname{Minimizar}} & f(\boldsymbol{\rho}, \boldsymbol{u}) \\
\text { Sujeito à } & \delta \Pi_{p}(\boldsymbol{\rho}, \boldsymbol{u}, \delta \boldsymbol{u})=\mathbf{0}, \quad \forall \delta \boldsymbol{u} \\
& \frac{\mathrm{V}\left(\boldsymbol{\rho}\left(\boldsymbol{x}^{\mathrm{r}}\right)\right)}{\mathrm{V}_{0}}-\bar{V} \leqslant 0 \\
& r_{\min } \leqslant r^{*}\left(\Omega_{\mathrm{d}}^{\mathrm{r}}\right)
\end{array}
$$

onde $f(\boldsymbol{\rho}, \boldsymbol{u})$ é a função objetivo do problema a ser minimizada. As restrições de projeto são dadas pela forma fraca do equilíbrio do sólido no espaço $\delta \Pi_{p}(2.20)$, da função de volume por $\bar{V}$ e da função de membro mínimo por $r_{\min }$ para o controle da complexidade da topologia respectivamente. A restrições de caixa das variáveis de projeto são dadas por $\rho\left(x^{r}\right) \in[\underline{\rho}, \bar{\rho}] \subset(0,1] \in \mathbb{R}^{+}$. Cada uma das restrições de projeto têm um papel importante na estabilidade do processo de otimização, sendo impostas em cada iteração do processo de iterativo de otimização baseado no método de ponto interior, para mais detalhes ver Seção 3.7 .

Neste trabalho o MOT é aplicado para sintetizar mecanismos flexíveis e atuadores devido a sua flexibilidade em conceber uma nova geometria ou topologia. É importante salientar que o termo $\mathrm{OT}$ restringe o verdadeiro escopo do método já que propriedades geométricas, como curvatura e área, também são determinados, sendo este um método que possui uma grande flexibilidade em sintetizar uma nova estrutura.

Para descrever a nova topologia $\left(\Omega_{d}^{r}\right)$ oMOT utiliza uma função de mistura das propriedades de materiais $\rho\left(x^{r}\right)$ dentro de um domínio fixo de projeto $\left(\Omega^{r}\right)$, denominado por domínio fixo estendido na configuração de referência que descreve uma nova geometria. Desta forma gerando uma região "pseudo-vazio" $\left(\Omega_{\mathrm{o}}^{r}\right)$ com propriedades muito inferior das demais regiões $\left(\Omega_{d}^{r}\right)$ (Bendsøe, 1989), como ilustrado na figura 4.1, tal que

$$
\Omega^{r}=\Omega_{d}^{r}+\Omega_{o}^{r}, \quad \rho\left(x^{r}\right)=\left\{\begin{array}{lll}
\bar{\rho} \leqslant 1.0 & \forall & x^{r} \in \Omega_{d}^{r} \\
\underline{\rho}>0.0 & \forall & x^{r} \in \Omega_{o}^{r}
\end{array}\right.
$$

Neste trabalho foi adotado o método das densidades utilizando o SIMP (Bendsøe, 1989) que será detalhado na Seção 4.1 e a técnica de projeção na Seção 4.4 proposta por 


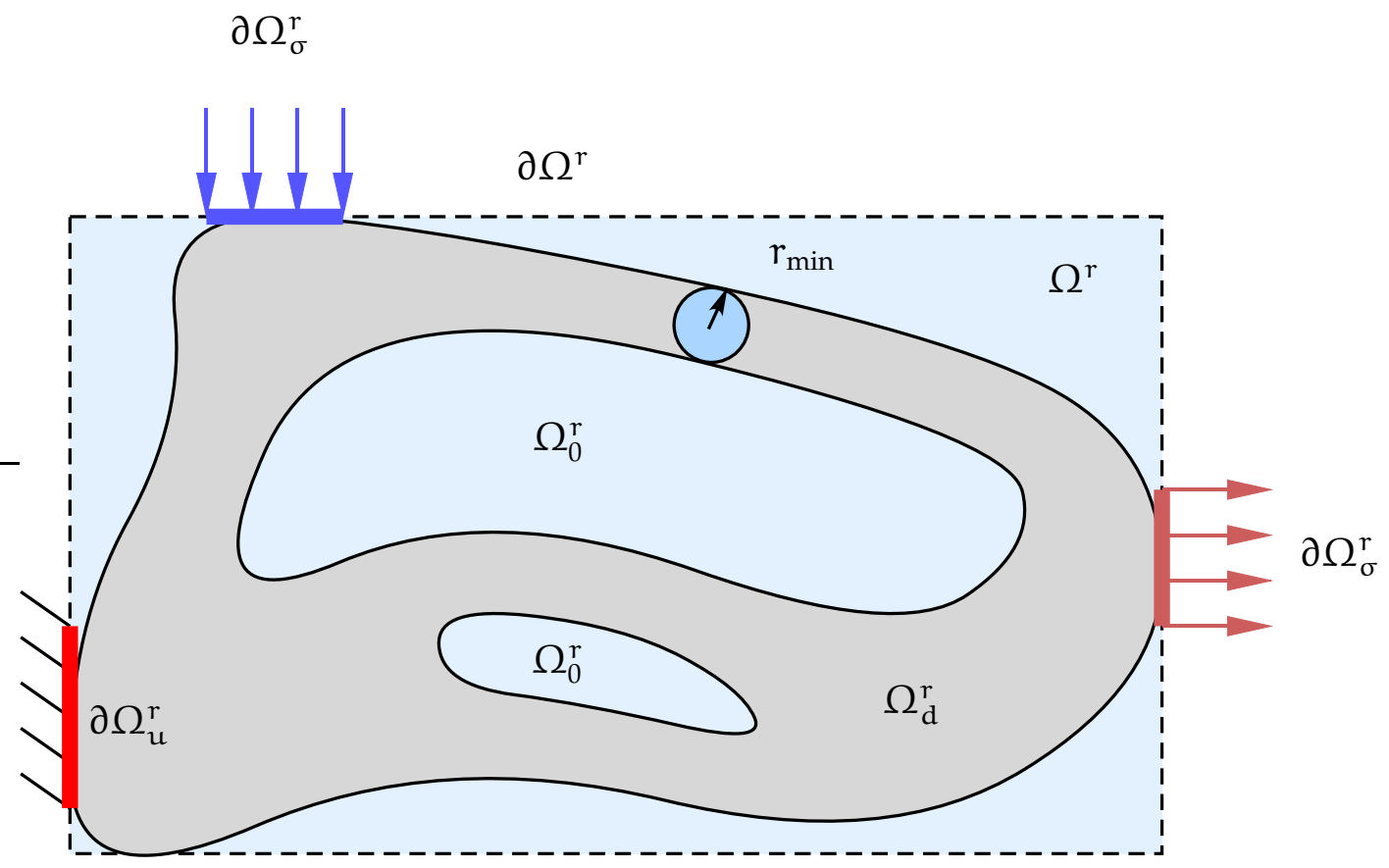

Figura 4.1: Topologia de um sólido deformável no MOT.

(Bourdin, 2001).

\subsection{Modelo de material - SIMP}

O MOT como já descrito procura distribuir material de forma otimizada no interior do domínio fixo estendido de projeto $\Omega^{\mathrm{r}}$ satisfazendo as equações de projeto (4.1) e para tal o método se utiliza do MEF para discretizar $\Omega^{r}$ em vários elementos finitos $(e)$ onde cada um deles se refere a uma variável que representam um ponto material no espaço. É importante mencionar que existem outros métodos equivalentes ao MEF que também podem ser utilizados em conjunto com MOT(Seo et al., 2010; Luo et al., 2013).

O modelo de material é aplicado na forma fraca do variacional do sistema multiplicando neste caso a função de energia de deformação específica de uma lei material (Lahuerta et al., 2013) em cada ponto material no espaço $\left(x^{r}\right)$ dentro do domínio $\Omega^{r}$ (ver Seções 2.2 $\mathrm{e} 2.5$ ). Esta alteração muda o comportamento físico do sólido permitindo assim que o processo iterativo de otimização, com as informações da sensibilidade do 
modelo material em relação as funções objetivo e de restrições separe os domínios $\Omega_{d}^{r}$ e $\Omega_{0}^{r}$. Entre os vários modelos de materiais utilizado no MOT) (Bendsoe e Sigmund, 2013), destaca-se o SIMP cuja sigla é uma abreviatura de "Solid Isotropic Material with Penalization" (Bendsøe, 1989), a função referente ao modelo material SIMP)é dada por:

$$
\varphi(\rho, \boldsymbol{u})=\rho\left(\boldsymbol{x}^{\mathrm{r}}\right) \varphi^{0}(\boldsymbol{u})
$$

onde as pseudo-densidades são integradas como uma função constante dentro de cada elemento finito $e$, logo:

$$
\rho_{e} \approx \frac{1}{|e|} \int_{\Omega^{r}} \rho\left(\mathbf{x}_{e}^{r}\right) d v_{e}^{r} .
$$

Substituindo as variáveis de projeto na função da energia de deformação específica (2.21), fica explícito na forma fraca do variacional $\delta \Pi_{p}$ onde o modelo de material recai sobre o tensor das tensões de engenharia $P(2.22)$, ou seja, no vetor de forças interna $\left(\mathbf{f}_{\text {int }}\right)(3.9)$, no tensor constitutivo material (3.4) e na matriz tangente material (3.12) $\left(\mathbf{K}_{M}\right)$ e assim por diante. Na equação (4.5) é apresentado de forma clara onde é aplicado o modelo de material no MOT como apresentado a seguir nas matrizes de elemento finito

$$
\begin{aligned}
{[\boldsymbol{P}]_{e} } & =\rho_{e}\left[\boldsymbol{P}^{0}\right]_{e}, & {\left[\boldsymbol{C}_{\mathbf{i j}}\right]_{e}=\rho_{e}\left[\boldsymbol{C}_{\mathbf{i j}}^{0}\right]_{e}, } \\
{\left[\mathbf{f}_{\text {int }}\right]_{e} } & =\rho_{e}\left[\mathbf{f}_{\text {int }}^{0}\right]_{e}, & {\left[\mathbf{K}_{M}\right]_{e}=\rho_{e}\left[\mathbf{K}_{M}^{0}\right]_{e} . }
\end{aligned}
$$

O SIMP sem um parâmetro de penalização ou com um valor igual um (1) permite a ocorrência de pseudo-densidades com valores intermediários, efeito conhecido como escala de cinza. Tal ocorrência torna o MOT incapaz de separar de forma discreta o domínio $\Omega^{r}$ nos domínios $\Omega_{0}^{r}$ para valores próximos de zero e $\Omega_{d}^{r}$ para valores iguais a um (1). No entanto, com a aplicação de um parâmetro de penalização exponencial em $\rho_{i}$ (Bendsøe, 1989; Lahuerta et al., 2013) permite mitigar a formação da escala de cinza na topologia

$$
\varphi(\rho, \boldsymbol{F})=\boldsymbol{\rho}\left(\boldsymbol{x}^{\mathrm{r}}\right)^{p} \varphi^{0}(\boldsymbol{F}),
$$

onde o expoente $p$ é referente a um fator de penalização que proporciona uma distribuição de material mais próxima de um caráter discreto ]0,1]. Na Figura 4.2 é possível visualizar o efeito do parâmetro de penalização sobre as pseudo-densidades em relação aos valores das propriedades físicas efetivas do material em relação a $\varphi(\rho, \boldsymbol{F})$. A penalização 


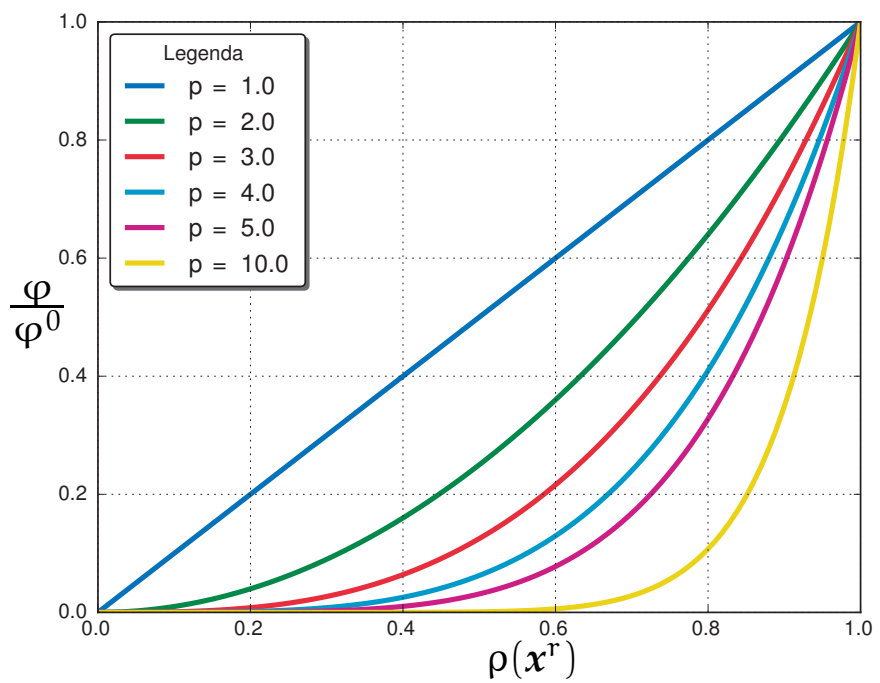

Figura 4.2: Influência do fator de penalização na propriedade efetiva da função de energia de deformação específica.

reduz o efeito dos valores intermediários sobre os valores propriedades efetivas do material. Nota-se que para valores altos de penalização, a curva da propriedade efetiva fica mais próxima de uma função "degrau", aproximando-se de uma distribuição discreta (Figura 4.3). Entretanto, valores muito elevados do fator de penalização levam aos problemas característicos de uma abordagem discreta ou seja o problema torna-se mal-posto novamente.

\subsection{Função de Continuação}

A maioria dos problemas de $\mathrm{OT}$ tem solução não convexa, acarretando na existência de mínimos locais dentro do espaço de solução. Portanto, a solução ótima é também dependente do valor de penalização utilizado. Isto significa que os parâmetros de penalização inicial e a ocorrência do seu incremento pode alterar a topologia final da estrutura. Tais resultados são apresentados por Lahuerta et al. (2013), onde é possível observar a ocorrência deste efeito. A ideia de utilizar uma função de continuação do parâmetro de penalização é evitar esta dependência no SIMP, alterando de forma gradualmente a seu valor, tornando desta forma o problema menos dependente dos valores iniciais de penalização no modelo material. A cada iteração o valor de "p"é 
gradativamente somado a um valor de $\Delta p$ assim sucessivamente até um valor limite:

$$
{ }^{(i+1)} p={ }^{(i)} p+\Delta p
$$

A literatura do MOT sobre elasticidade linear é comum utilizar um valor inicial de penalização constante $p=2$ ou $p=3$. No trabalho de Kemmler et al. (2005) foi demonstrado, a partir de exemplos numéricos, que o valor $p=2$ apresenta resultados melhores em termos de valor final da função objetivo quando aplicados em problemas de maximização da rigidez. No entanto, o trabalho de Lahuerta et al. (2013), foi apresentado exemplos numéricos onde $p=2$ também acarreta em soluções sub-ótimas e que o ideal é iniciar o processo iterativo de otimização com o valor inicial de penalização $p=1$ e de modo incremental alterar seu valor através de uma função de continuação.

\subsection{Problemas associados à discretização no MOT}

$\mathrm{O}$ MOT possui alguns problemas relativos ao modo que é implementado numericamente, como dependência de malha, instabilidade de tabuleiro, escala de cinza. Como já mencionado no item 4.1 em problemas tradicionais de OT onde se busca obter uma distribuição discreta ótima de material ao longo do domínio fixo estendido de projeto $\left(\Omega^{r}\right)$ pode se apresentar de forma suave e contínuo ao longo do domínio, resultando em valores intermediários entre os dois limites da restrição de caixa do problema de otimização. A este fenômeno é dado o nome de "escala de cinza", como é apresentado na figura 4.3 (ver seção 4.1). Os resultados com escala de cinza são consistentes do ponto de vista numérico, geralmente alcançam maior eficiência, mas do ponto de vista de engenharia ainda são pouco factíveis pelos processos de manufatura atuais, porém com o aperfeiçoamento das impressoras 3Ds em breve será possível ter resultados com escala de cinza a um custo competitivo e com uma aplicação regular na Engenharia. Outro problema relacionado ao MOTé referente a instabilidade de tabuleiro (ou em inglês checkerboard) que é caracterizada pela formação de regiões com material e sem material ou com baixo valor de pseudo-densidade próximo do valor da restrição de caixa, dispostos em 


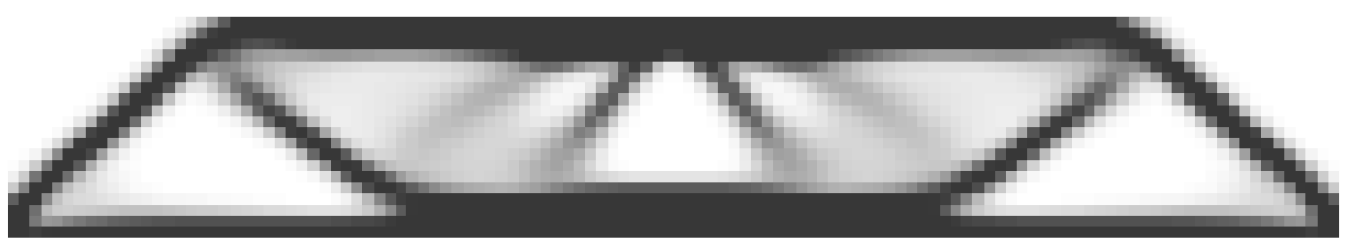

Figura 4.3: Representação da escala de cinza.

um formato de tabuleiro como ilustrado na Figura 4.4. Este tipo de instabilidade é mais recorrente em problemas de otimização estrutural onde a função objetivo que se deseja minimizar está relacionada com a função de flexibilidade do modelo. Nestes casos, a configuração padrão de tabuleiro apresenta numericamente uma maior rigidez (rigidez artificial) do que uma configuração de mesmo volume porém homogênea, sendo neste caso predominantes em algumas regiões ao final do processo de OT. Conforme demonstrado por Haber et al. (1996), a natureza do surgimento do padrão checkerboard é a mesma envolvida na convergência do MEF. Trata-se de uma incompatibilidade entre a função de forma do campo dos deslocamentos em relação ao campo das pseudo-densidades, que provocam o surgimento de uma distribuição de oscilações espúrias das variáveis de projeto.

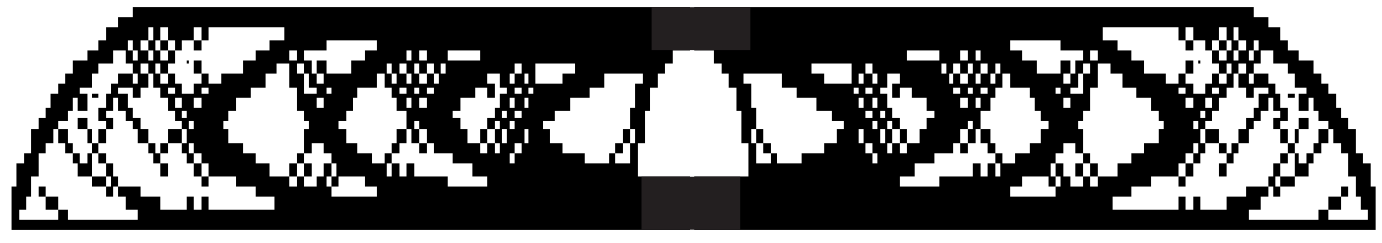

Figura 4.4: Resultado com instabilidade de tabuleiro.

\subsection{Função de Projeção}

A função de projeção foi inicialmente proposta por Bourdin (2001) para que fosse possível impor uma restrição indireta de perímetro mínimo local, eliminando problemas de dependência de malha e de instabilidade de tabuleiro. É uma função de regularização que consiste em projetar um domínio pseudo-densidades sobre um domínio de variáveis regularizadas dentro do domínio de projeto através de uma integral de convolução, 
operando desta forma como um filtro.

A operação de regularização via integral de convolução utilizada para obter as variáveis de projeto é dada por:

$$
\widehat{\rho}\left(\bar{x}^{\mathrm{r}}\right):=(G * \rho)\left(\bar{x}^{\mathrm{r}}\right)=\int_{\mathbb{R}^{\mathrm{n}}} G\left(x^{\mathrm{r}}-\bar{x}^{\mathrm{r}}\right) d x^{\mathrm{r}}, \quad G \in \mathcal{H}_{1}
$$

onde um ponto material $\bar{x}^{r} \in \Omega^{r} \in \mathbb{R}^{n}$ no centro uma esfera de raio $r_{\text {min }}>0$ representada pelo subdomínio $\bar{\Omega}^{r} \subset \Omega^{r}$, logo a função de ponderação $G \geqslant 0 \Leftrightarrow \subset \bar{\Omega}^{r} \operatorname{logo}$ sua integral tem o seguinte valor:

$$
w\left(\bar{x}^{r}\right):=\int_{\bar{\Omega}^{r}} G d x^{r}=1
$$

Portanto, cada ponto material $x^{r}$ tem-se uma função de regularização suave e diferenciável em $\mathcal{H}_{1}$ que representam o campo das variáveis de projeto, onde $\rho \rightarrow \widehat{\rho}$, também denominadas de variáveis topológicas. Consequentemente as propriedades físicas da função de energia de deformação específica $\varphi$ (2.21) dependeram da função de regularização $G$.

Aplicando a função de regularização (4.8) na forma discreta sobre o domínio $\Omega^{r}$ aproximado pelo MEF obtemos a seguinte função $\widehat{\rho}_{e}$ em um elemento finito arbitrário e:

$$
\widehat{\rho}_{e}:=\left(G * \rho_{e}\right)\left(\mathbf{x}_{e}^{r}\right)=\frac{\sum_{j \subset \bar{\Omega}_{e}^{r}} \rho_{j} \int_{j} G\left(\mathbf{x}_{j}^{r}-\mathbf{x}_{e}^{r}\right) d \mathbf{x}^{r}}{\sum_{j \subset \bar{\Omega}_{e}^{r}} \int_{j} G\left(\mathbf{x}_{j}^{r}-\mathbf{x}_{e}^{r}\right) d \mathbf{x}^{r}}=\frac{\sum_{j \subset \bar{\Omega}_{e}^{r}} \rho_{j} \bar{w}_{j}}{\sum_{j \subset \bar{\Omega}_{e}^{r}}^{r} \bar{w}_{j}}=\frac{\overline{\boldsymbol{\rho}}_{e} \cdot \overline{\mathbf{w}}_{e}}{\overline{\mathbf{w}}_{e}}
$$

onde $\overline{\boldsymbol{\rho}}_{e}, \overline{\mathbf{w}}_{e}, \mathbf{x}_{e}^{\mathrm{r}}$ e $\mathbf{x}_{j}^{\mathrm{r}}$ são os vetores que contém o campo das pseudo-densidades, dos pesos de ponderação, das coordenadas do centroide de um elemento arbitrário e e das coordenadas do centroide dos elementos arbitrários $j$ dentro do subdomínio $\bar{\Omega}_{e}^{r}$ como ilustrado pela Figura 4.5. Portanto, quanto maior for à distância de um elemento arbitrário j do centro da esfera $\bar{\Omega}_{e}^{r}$, menor será a sua influência em $\widehat{\rho}_{e}$, de modo que, quanto mais próximo do centro, maior será a influência.

A função de ponderação $G$ na forma discreta pode ser apresentada da seguinte 


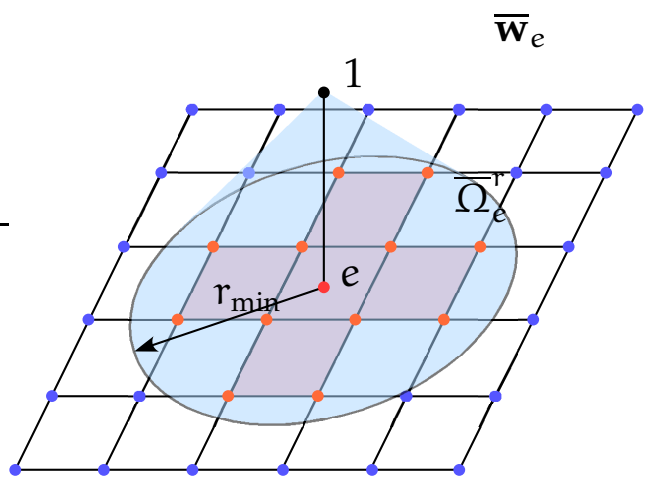

Figura 4.5: Representação do raio de influência $r_{\min }$ considerando a penalização a função de ponderação $\overline{\mathbf{w}}_{e}$.

forma:

$$
\overline{\mathbf{w}}_{e}=\left\{\begin{array}{ll}
\frac{r_{\min }-\mathbf{r}_{e}}{r_{\min }} & \text { se } \mathbf{x}_{j}^{r} \subset \bar{\Omega}_{e}^{r} \\
0 & \text { se } \mathbf{x}_{j}^{r} \not \subset \bar{\Omega}_{e}^{r}
\end{array}, \quad \text { onde } \quad \mathbf{r}_{e}=\left\|\mathbf{x}_{j}^{r}-\mathbf{x}_{e}^{r}\right\| \leqslant r_{\min } .\right.
$$

Aplicando um parâmetro de penalização exponencial $p$ na função de ponderação $G$ é possível controlar a influência dos elementos dentro do perímetro de projeção $\bar{\Omega}_{e}^{r}$. O parâmetro $p$ pode ser utilizado ou atualizado em conjunto com a função de continuação do modelo material (ver Seção 4.2) ou seja em conjunto com o parâmetro de penalização do SIMP (4.6) com a finalidade de reduzir a escala no resultado final. Esta técnica de penalizar a função de ponderação $G$ demonstrou eficiência na eliminação da escala de cinza, porém também removeu o efeito da projeção ao longo do processo OTacarretando na dependência de malha se o valor do parâmetro de penalização $p$ for muito elevado.

A vantagem desta função de projeção está na sua simplicidade, pois, não necessita de restrições adicionais, garantindo a existência de solução para $\rho \rightarrow \widehat{\rho}$ pois está associado a convergência do MEF(Bourdin, 2001). Na Figura 4.6é possível verificar a independência dos resultados utilizando a técnica de projeção sobre uma viga engastada de 1000 × 250 mm de domínio de projeto. 

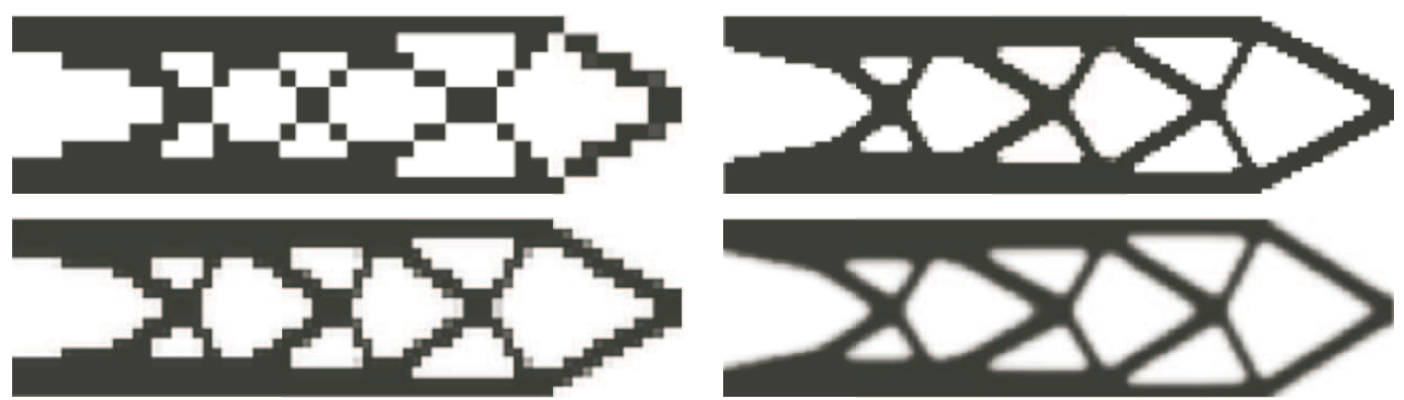

Figura 4.6: Resultados de topologia ótima para diferentes tamanhos de elementos para uma viga engastada de dimensões de $1000 \times 250 \mathrm{~mm}$, para $r_{\min }=30$ com uma discretização de $175,1250,2500$ e 5000 elementos respectivamente.

\subsection{Análise de Sensibilidade}

O problema de otimização topológica neste projeto foi resolvido de forma separada do equilíbrio do sistema (3.10), o que é chamado na literatura de solução segmentada ou "Nested Approach" (Haftka e Gürdal, 2012). Inicialmente é resolvido o problema do equilíbrio (3.10), utilizando algum método iterativo de solução e então com os vetores dos deslocamentos nodais é possível calcular a sensibilidade da função objetivo e restrições do problema para resolver o problema da distribuição de material ao longo do domínio fixo estendido de projeto utilizando um algoritmo de otimização. Neste trabalho foi utilizado o algoritmo de ponto interior, cujo método é descrito na Seção 3.8 .

\subsubsection{Energia de Deformação Externa}

A função da energia de deformação específica no domínio estendido de projeto $\left(\omega^{r}\right)$ está conectada carregamentos externos aplicados ao modelo e pode ser denominada também como a flexibilidade no ponto de aplicação do carregamento ou em inglês por 'End Compliance'.

Esta funcão foi muito utilizada em diversos artigos (Buhl et al., 2000; Bruns e Tortorelli, 2001; Lahuerta et al., 2013) com aplicações do MOT envolvendo não linearidade elástica ou geométrica devido a simplicidade de obter a sua primeira derivada em relação as variáveis de projeto $\rho_{i}$. Podemos definir então a função de flexibilidade externa no 
domínio estendido de projeto por:

$$
\mathrm{U}_{\mathrm{ext}}(\boldsymbol{\rho}, \boldsymbol{u})=\int_{\partial \Omega^{\mathrm{r}}} \boldsymbol{t}^{\mathrm{r}} \cdot \boldsymbol{u} d a^{\mathrm{r}}+\int_{\Omega^{\mathrm{r}}} \boldsymbol{b}^{\mathrm{r}} \cdot \boldsymbol{u} d v^{\mathrm{r}} \approx \mathbf{f}_{\mathrm{ext}} \cdot \mathbf{u},
$$

onde $\mathbf{f}_{\text {ext }}$ é referente ao vetor dos carregamentos externos e $\mathbf{u}$ o vetor dos deslocamento, portanto o seu produto interno é igual a energia de deformação originada pelo carregamento externo. Derivando a equação (4.11) em relação a variável de projeto, obtemos:

$$
\begin{aligned}
\frac{\partial \mathbf{U}_{\mathrm{ext}}}{\partial \rho_{\mathrm{i}}} & =\frac{\partial\left(\mathbf{f}_{\mathrm{ext}} \cdot \mathbf{u}\right)}{\partial \rho_{\mathrm{i}}}=\frac{\partial \mathbf{f}_{\mathrm{ext}}}{\partial \rho_{\mathrm{i}}} \cdot \mathbf{u}+\mathbf{f}_{\mathrm{ext}} \cdot \frac{\partial \mathbf{u}}{\partial \rho_{\mathrm{i}}}=\mathbf{0}+\mathbf{f}_{\mathrm{ext}} \cdot\left(\frac{\partial \mathbf{u}}{\partial \rho_{\mathrm{i}}}\right) \\
& =\mathbf{f}_{\mathrm{ext}} \cdot\left(\frac{\partial \mathbf{u}}{\partial \mathbf{r}} \frac{\partial \mathbf{r}}{\partial \rho_{\mathrm{i}}}\right)=\mathbf{f}_{\mathrm{ext}} \cdot\left(\mathbf{K}_{\mathrm{T}}^{-1} p \int_{\Omega^{r}} \rho_{i}^{p-1} \mathbf{N}_{, j}^{\top} \boldsymbol{\tau}_{\mathbf{j}}^{0} d v^{\mathrm{r}}\right) \\
& =\underbrace{\left(\mathbf{f}_{\mathrm{ext}}^{\top} \mathbf{K}_{\mathrm{T}}^{-1}\right)}_{\beta} \cdot p \int_{\Omega^{r}} \rho_{i}^{-1} \mathbf{N}_{, j}^{\top} \boldsymbol{\tau}_{j} d v^{r} .
\end{aligned}
$$

Isolando em (4.12) o termo $\beta$ em relação a variável de projeto $\rho_{i}$

$$
\frac{\partial \mathrm{U}_{\mathrm{ext}}}{\partial \rho_{\mathrm{i}}}=\boldsymbol{\beta}_{\mathrm{i}} \cdot p \int_{\Omega^{\mathrm{r}}} \rho_{i}^{-1} \mathbf{N}_{, j}^{\top} \boldsymbol{\tau}_{\mathrm{j}} d v^{\mathrm{r}}
$$

\subsubsection{Deslocamento}

A derivada do vetor dos deslocamentos u dado pela equação (3.5) em relação a variáveis de projeto $\rho_{j}$ é obtida por:

$$
\frac{\partial \mathbf{u}}{\partial \rho_{j}}=\frac{\partial \mathbf{u}}{\partial \mathbf{r}} \frac{\partial \mathbf{r}}{\partial \rho_{j}}=\mathbf{K}_{\mathrm{T}}^{-1} \frac{\partial}{\partial \rho_{j}}\left(\mathbf{f}_{\text {int }}-\mathbf{f}_{\mathrm{ext}}\right)=\mathbf{K}_{\mathrm{T}}^{-1} p \int_{\Omega^{\mathrm{r}}} \rho_{\mathrm{i}}^{\mathrm{p}-1} \mathbf{N}_{, \mathrm{k}}^{\top} \boldsymbol{\tau}_{\mathrm{k}}^{0} d v^{\mathrm{r}},
$$

no entanto a dimensão de (4.14) não é compatível com derivada de um grau de liberdade i em específico. Para tal, é preciso selecionar o grau de liberdade desejado multiplicando (4.14) por um vetor de seleção $\mathbf{h}_{i}$ dos deslocamentos em região do contorno $\partial \Omega_{\mathfrak{u}}^{r}$

$$
\frac{\partial u_{i}}{\partial \rho_{j}}=\mathbf{h}_{i} \cdot \frac{\partial \mathbf{u}}{\partial \rho_{j}}=\underbrace{\mathbf{h}_{i}^{\top} \mathbf{K}_{\mathrm{T}}^{-1}}_{\boldsymbol{\beta}_{i}} p \int_{\Omega^{r}} \rho_{j}^{p-1} \mathbf{N}_{, k}^{\top} \boldsymbol{\tau}_{k}^{0} d v^{r}=\left[\boldsymbol{\beta}_{i}\right] \cdot p \int_{\Omega^{r}} \rho_{j}^{p-1} \mathbf{N}_{, k}^{\top} \boldsymbol{\tau}_{k}^{0} d v^{r} .
$$




\subsubsection{Fator de Incremento de carga}

Em problemas relacionados a configuração de equilíbrio crítica, o fator de carregamento é desconhecida, sendo uma variável a mais no problema a ser determinada, além dos deslocamentos. Para contorna esta dificuldade e obter uma derivada que relaciona o carregamento proporcional em relação às variáveis de projeto Noguchi e Hisada (1993) propôs utilizar a restrição de arco cilíndrica em relação a configuração de referência.

A ideia está relacionada com o gradiente do carregamento proporcional, que é estimado através da busca de uma configuração pseudo-crítica ou pseudo-instável dentro de uma restrição de arco cilíndrico aplicando-se uma perturbação na configuração de equilíbrio do sistema através das variáveis de projeto $\rho_{j}$. A restrição de arco cilíndrica em relação a posição final do sistema pode ser escrita como:

$$
\mathbf{u} \cdot \mathbf{u}=\text { constante, }
$$

e diferenciando em relação as variáveis de projeto, obtemos a relação de ortogonalidade:

$$
\mathbf{u} \cdot \frac{\mathrm{d} \mathbf{u}}{\mathrm{d} \rho_{\mathrm{j}}}=0
$$

Diferenciando a equação do comprimento de arco (3.40) que rege a atualização dos deslocamentos para o MCGD e MCA, obtemos:

$$
\frac{d \mathbf{u}}{d \rho_{j}}=\frac{\partial \mathbf{u}_{r}}{\partial \rho_{j}}+\frac{d \bar{\lambda}}{d \rho_{j}} \mathbf{u}_{0}
$$

onde $\partial \mathbf{u}_{\mathrm{r}} / \partial \rho_{\mathrm{j}}$ é referente a derivada parcial do vetor dos deslocamentos em relação as variáveis de projeto, onde é dado pela equação (4.14) e aplicando a condição de ortogonalidade apresentada pela equação (4.17), chega-se na seguinte relação:

$$
\mathbf{u} \cdot \frac{d \mathbf{u}}{d \rho_{j}}=\mathbf{u} \cdot \frac{\partial \mathbf{u}_{r}}{\partial \rho_{j}}+\frac{d \bar{\lambda}}{d \rho_{j}} \mathbf{u} \cdot \mathbf{u}_{0}=0
$$

Igualando-se a zero, é possível isolar $d \bar{\lambda} / d \rho_{j}$ e multiplicando ambos os lados por $\mathbf{u}_{0}$ 
obtendo:

$$
\frac{\mathrm{d} \bar{\lambda}}{\mathrm{d} \rho_{\mathrm{j}}}=-\frac{\mathbf{u} \cdot \partial \mathbf{u}_{\mathrm{r}} / \partial \rho_{\mathrm{j}}}{\mathbf{u} \cdot \mathbf{u}_{0}}=-\frac{\mathbf{u} \cdot \mathbf{K}_{\mathrm{T}}^{-1} \partial \mathbf{r} / \partial \rho_{j}}{\mathbf{u} \cdot \mathbf{K}_{\mathrm{T}}^{-1} \mathbf{f}_{\mathrm{ext}}}=-\frac{\mathbf{v} \cdot \partial \mathbf{r} / \partial \rho_{\mathrm{j}}}{\mathbf{v} \cdot \mathbf{f}_{\mathrm{ext}}}
$$

É interessante mencionar que a equação (4.20) é muito similar a equação da derivada da flambagem não linear proposta por Wu e Arora (1988) por:

$$
\frac{\mathrm{d}^{w} \bar{\lambda}}{\mathrm{d} \rho_{\mathrm{j}}}=\frac{\boldsymbol{\phi}_{\mathrm{i}} \cdot \partial \mathbf{u}_{\mathrm{r}} / \partial \rho_{j}}{\boldsymbol{\phi}_{\mathrm{i}} \cdot \mathbf{f}_{\mathrm{ext}}}
$$

No entanto, a equação (4.21) só será valida ou terá efetividade quando a estrutura estiver próximo de um ponto instável na trajetória de equilíbrio, também denominado de ponto de carregamento crítico (c), $\bar{\lambda}={ }^{c} \bar{\lambda}$.

Teste numéricos, apresentam evidencias que próximo ao carregamento crítico ${ }^{c} \bar{\lambda}, o$ vetor $\mathbf{v}$ se aproxima do autovetor $\boldsymbol{\phi}_{i}$ ou do modo de flambagem conforme:

$$
\frac{{ }^{c} \mathbf{v}}{\left\|{ }^{c} \mathbf{v}\right\|} \approx \frac{\phi_{i}}{\left\|\phi_{i}\right\|}
$$

atestando uma relação entre o modo do autovetor $(i)$ e o ponto de carregamento crítico (c), indicando que ambas as formulações são similares na configuração crítica. Entretanto, a formulação de Noguchi e Hisada (1993) é mais ampla, pois, permite a utilização de uma configuração fora da crítica, não necessitando do cálculo dos autovalores e autovetores da estrutura.

\subsubsection{Volume}

A função que descreve o volume total do domínio fixo estendido $\left(\overline{\mathrm{V}}^{\mathrm{r}}\right)$ em relação as variáveis de projeto $\left(\rho_{i}\right)$ é dado pela somatória da integral de cada elemento finito $(i)$ dentro do domínio de projeto $\left(\Omega^{r}\right)$, descrito por:

$$
\int_{\Omega_{i}^{r}} \rho_{i} d v_{i}^{r} \cong \sum_{i=1}^{n_{e}} \rho_{i} v_{i}^{r}=\sum_{i=1}^{n_{e}} \bar{v}_{i}^{r}=\bar{V}^{r},
$$


portanto a sensibilidade em relação as variáveis de projeto $\rho_{e}$ é obtida da seguinte forma:

$$
\frac{\partial \bar{V}^{r}}{\partial \rho_{e}}=\frac{\partial\left(\rho_{i} \bar{v}_{i}^{r}\right)}{\partial \rho_{e}}=\delta_{e i} \bar{v}_{i}^{r}=\bar{v}_{e}^{r}
$$

\subsection{Sínteses de Mecanismos Flexíveis via Método de Otimização Topológica}

Um mecanismo flexível ou flextensional geralmente é projetado para trabalhar no regime elástico do material do qual é constituído. Para tanto, sua flexibilidade é utilizada como meio para transformar a energia $\left(W_{\text {in }}\right)$ na porta de entrada $\left(\partial \Omega_{\mathfrak{i n}}^{r}\right)$ em deslocamento ou força na porta $\left(\mathbf{u}_{\mathrm{ou}}, \mathbf{f}_{\mathrm{in}}\right)$ de saída $\left(\left(\partial \Omega_{\mathrm{ou}}^{\mathrm{r}}\right)\right)$, conforme descrição da figura 4.7. Estas

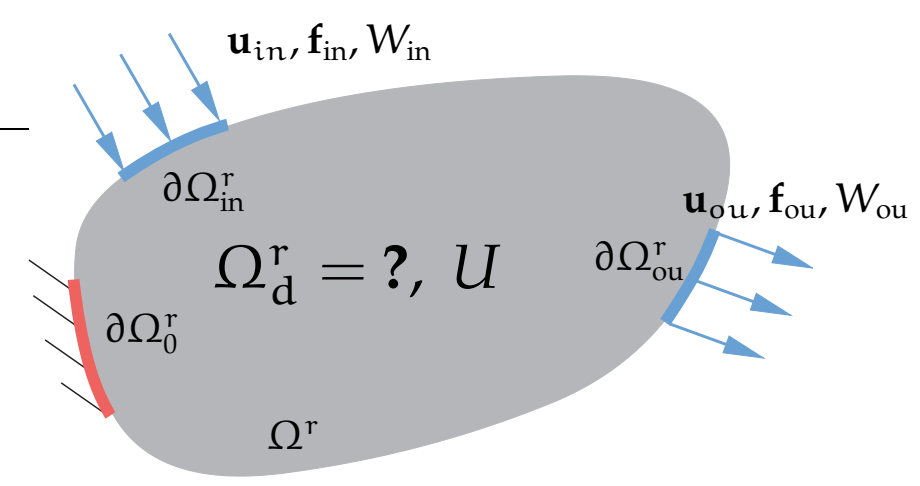

Figura 4.7: Representação do domínio de projeto de um mecanismo flexível.

estruturas podem tem uma ou mais portas de entrada e saída, dependendo somente dos requisitos de projeto. Considerando neste trabalho que o sistema seja conservativo e quase-estático, a energia na porta de entrada é igual à soma da energia interna $(U)$ da estrutura com o trabalho da porta de saída do mecanismo $\left(W_{\mathrm{ou}}\right)$.

$$
W_{\text {in }}=W_{\text {ou }}+U,
$$


onde $W_{\text {in, }} W_{\text {ou }}$ e são $U$ são dados por por:

$$
\begin{aligned}
W_{\text {in }} & =\mathrm{u}_{\mathrm{ext}}=\mathbf{f}_{\mathrm{in}} \cdot \mathbf{u}_{\mathrm{in}}, \\
W_{\mathrm{ou}} & =\mathbf{f}_{\mathrm{ou}} \cdot \mathbf{u}_{\mathrm{ou}}, \\
u & =W_{\mathrm{in}}-W_{\mathrm{ou}} .
\end{aligned}
$$

Os vetores $\mathbf{f}_{\text {ou }}$ e $\mathbf{f}_{\text {in }}$ são referentes a força na porta de saída e entrada do mecanismo $\left(\partial \Omega_{\mathrm{ou}}^{\mathrm{r}}\right.$ e $\left.\partial \Omega_{\mathrm{in}}^{\mathrm{r}}\right)$, e $U$ é referente a energia interna retida pelo mecanismo. O mecanismo pode ser projetado com base na relação entre as forças na porta de entrada e saída ou coeficiente de eficiência mecânica (ou em inglês denominado de MA), apresentado por:

$$
M A=\sum \mathbf{f}_{\mathrm{in}} \oslash \mathbf{f}_{\mathrm{ou}}=\frac{\left\|\mathbf{f}_{\mathrm{in}}\right\|_{1}}{\left\|\mathbf{f}_{\mathrm{ou}}\right\|_{1}}
$$

Outro importante parâmetro de eficiência reside na relação entre os deslocamentos na porta de entrada e de saída, denominado por coeficiente de eficiência geométrica (ou em inglês denominado de (GA) e dado por:

$$
G A=\sum \mathbf{u}_{\mathrm{ou}} \oslash \mathbf{u}_{\mathrm{in}}=\frac{\left\|\mathbf{u}_{\mathrm{ou}}\right\|_{1}}{\left\|\mathbf{u}_{\mathrm{in}}\right\|_{1}}
$$

Portanto o projeto de tais estruturas devem levar em consideração os coeficientes MA e GA para obter uma relação de redução, ampliação de forças ou de deslocamento nas portas de entrada e de saída, dispondo de uma relação inversamente proporcional entre os coeficientes.

A implementação do MOTneste trabalho não inclui elementos ou variáveis de rotação e translação como mola ou treliça, consequentemente as ligações internas da estrutura são constituídas basicamente de vigas, barras e dobradiças formadas por elementos contínuos, que serão responsáveis pela flexibilidade da estrutura ao longo do domínio de projeto $\left(\Omega^{r}\right)$, também denominadas em inglês por "hinges".

No Capítulo 4.6.1 é apresentada uma formulação geral para a síntese de atuadores flextensionais que independe da hipótese cinemática ou elástica do domínio de projeto. 


\subsubsection{Minimização da Energia de Deformação Interna com Restrições via Método dos Mínimos Quadrados}

A ideia de projetar mecanismos flexíveis impondo restrições de força interna e deslocamento via uma modificação do método dos mínimos quadrados apresentado na Seção 3.9 foi baseado nas conclusões e métodos apresentados pelos trabalhos de Bruns et al. (2002a); Bruns e Sigmund (2004); Ohsaki e Nishiwaki (2005); Lindgaard e Dahl (2013) cuja conclusão implícita foi a dificuldade de se impor uma restrição de deslocamento e de incremento de carga $\bar{\lambda}$ na síntese de estruturas que utilizam o mecanismo de pós-flambagem. Aparentemente os algoritmos de otimização utilizados (CO, MAM, PLS, PQS, ver Seção.3e Apêndice para mais detalhes) não foram capazes de obter uma ótima distribuição das variáveis de projeto de forma a satisfazer as restrições, no caso os deslocamentos, sujeito a uma restrição não linear de equilíbrio do sistema mecânico. Equilíbrio este necessário para resolver o problema de elasticidade não-linear (3.10) que envolve também a questões relativas as regiões com baixo valor de pseudo-densidade. Uma descrição mais detalhada sobre este tema é apresentada por Lahuerta et al. (2013), portanto, a formulação geral do problema de OT proposta para evitar todos estes problemas relatados é apresentada:

$$
\begin{array}{ll}
\underset{\rho_{i} \in[0.0,1.0]}{\operatorname{Min} .} & \Theta \\
\text { Sujeito à } & \delta \Pi_{p}\left(\rho_{i}, u, \delta u\right)=0 \\
& \boldsymbol{\alpha}\left(\rho_{i}\right)-\underline{\alpha}=0 \\
& \bar{V}^{r}\left(\rho_{i}\right)-\underline{v} \leqslant 0 \\
& r_{\min } \leqslant r^{*}\left(\Omega_{d}^{r}\right)
\end{array}
$$

onde $\Theta$ é uma função relacionada a somatória da flexibilidade (4.30) de caso de carregamento (lc) é descrita da seguinte forma:

$$
\Theta=\sum_{l c}^{n_{l c}} \mathbf{f}_{\mathrm{ext}}^{(\mathrm{lc})} \cdot \mathbf{u}^{(\mathrm{lc})} .
$$

As restrições de igualdade são dadas pelo vetor $\alpha$ que representam os valores das funções e $\underline{\alpha}$ o vetor com os valores desejados ou os valores de igualdade. Este vetor 
de restrições pode conter os deslocamentos ou incremento de carga desejados na porta de entrada e/ ou saída do mecanismo. Também pode-se utilizar para obter um valor específico de MA GA. Logo podemos reescrever (4.29) incluindo as restrições via MMQ por:

$$
\begin{array}{ll}
\operatorname{Min}_{\rho_{i} \in[0.0,1.0]} & \sum_{l c}^{n_{l c}} \mathbf{f}_{\text {ext }}^{(l c)} \cdot \mathbf{u}^{(l c)}+\sum_{n=1}^{m} \frac{1}{2}\left(\frac{\alpha_{n}\left(\rho_{i}\right)-\underline{\alpha}_{n}}{w_{n}}\right)^{2} \\
\text { Sujeito à } & \delta \Pi_{p}\left(\rho_{i}, u, \delta u\right)=0 \\
& \bar{V}^{r}\left(\rho_{i}\right)-\underline{v} \leqslant 0 \\
& r_{\min } \leqslant r^{*}\left(\Omega_{d}^{r}\right)
\end{array}
$$

O conceito de impor as restrições via $\mathrm{MMQ}$ é permitir uma relaxação das restrições criando domínio viável alargado e desta forma obter uma distribuição de material que satisfaça a condição de equilíbrio dada pela equação (3.10), pois, a imposição de restrições inicialmente fora do domínio viável $F$ (4.31) geram instabilidades durante o processo de otimização, sendo esta uma das razões das dificuldades se impor as restrições relatadas pelos trabalhos de Bruns e Sigmund (2004); Ohsaki e Nishiwaki (2005).

\subsection{Implementação do MOT}

A implementação deste trabalho utilizou-se de derivadas de primeira ordem das funções objetivo e de restrições, utilizando as derivadas descritas nas seções 4.5.1, 4.5.2, 4.5.3, 4.5.4. Sendo o algoritmo de otimização numérico parte essencial do processo de OT, conforme fluxograma apresentado na figura 4.8, respectivamente. 


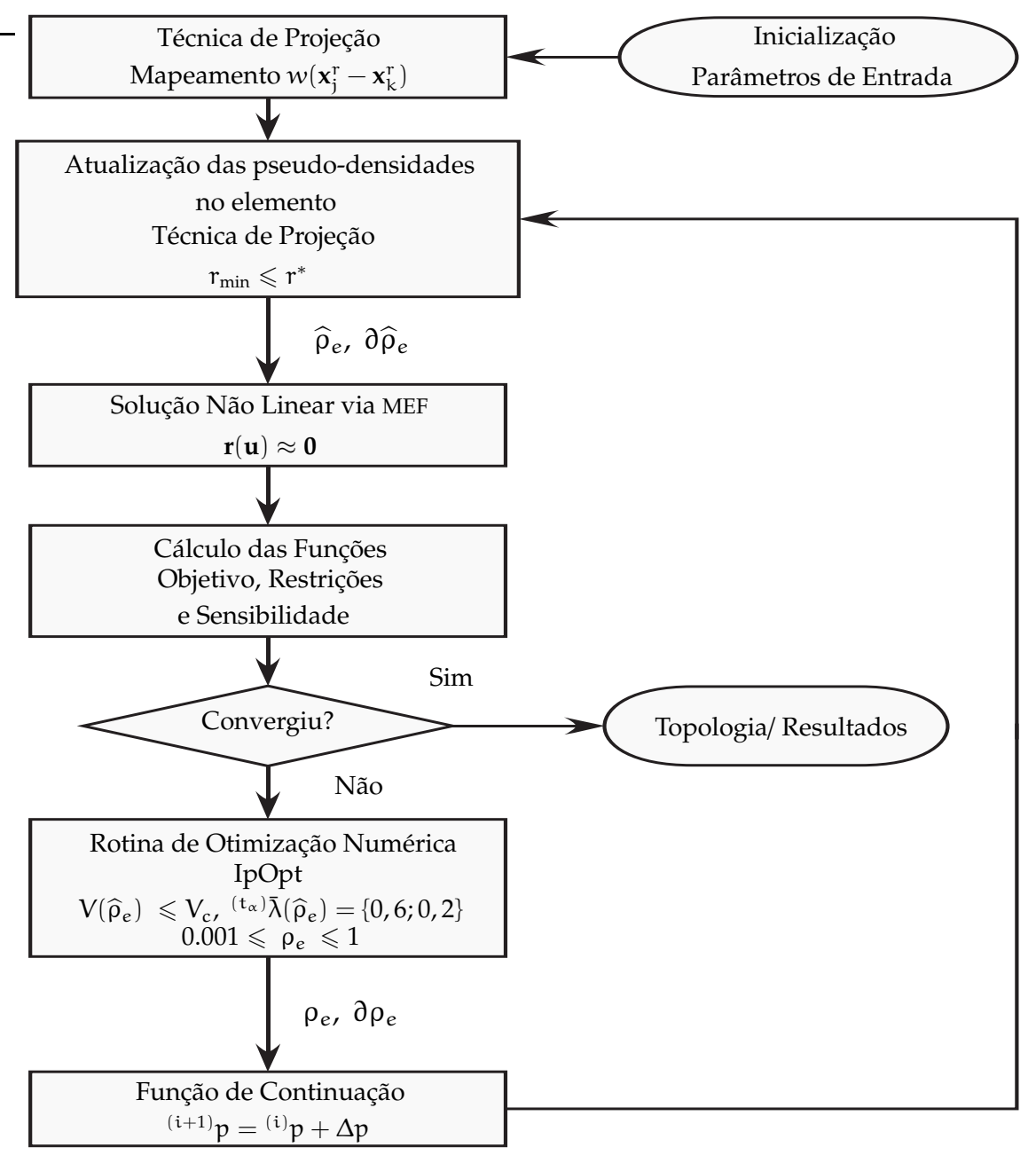

Figura 4.8: Generalização do algorítimo de OT 


\section{Capítulo 5}

\section{Resultados}

Os resultados apresentados neste trabalho são baseados na síntese de atuadores flextensionais, considerando como objeto de projeto, obter mecanismos flexíveis desses atuadores que trabalhem utilizando o mecanismo de flambagem não linear. A porta de entrada do mecanismo é representada por "in"e de saída por "ou"no domínio $\partial \Omega^{\text {r }}$ respectivamente. As propriedades elásticas do material utilizado são apresentadas na tabela 5.1 .

Tabela 5.1 - Propriedade elásticas do material utilizado

\begin{tabular}{ccc}
\hline Propriedades & valor & unidade \\
\hline Módulo de Elasticidade $(E):$ & 1,00 & $\mathrm{GPa}$ \\
Coeficiente de poisson $(v):$ & 0,40 & - \\
\hline
\end{tabular}

Lembrando que as constantes de Lamé em relação as propriedades dada na tabela 5.1 são apresentadas:

$$
\lambda=\frac{E v}{(1+v)(1-2 v)}, \quad \mu=\frac{E}{2(1+v)} .
$$




\subsection{Mecanismo Flexível Biestável}

Nesta seção, é apresentada a formulação de mecanismos flexíveis biestáveis desenvolvida neste trabalho, ou seja, busca-se uma estrutura que possua duas posições de equilíbrio estáveis $\left\{0 ; t_{1}\right\},\left\{t_{2} ; 1,0\right\}$ separados por uma região de equilíbrio instável $\left\{t_{1} ; t_{2}\right\}$. Tais mecanismos têm aplicação em atuadores biestáveis, válvulas comutadoras, dispositivos de acionamento "liga/desliga", stents cardíacos, por exemplo. Necessitam de pouca energia para operar, pois, somente alteram seu estado de equilíbrio (liga/desliga) sob uma força externa, não necessitando, portanto, de fonte de energia extra para se manter atuado (ligado) na porta de saída.

Na figura 5.1 são apresentadas as condições de contorno $\partial \Omega_{\mathfrak{u}}^{r}$ e $\partial \Omega_{\sigma}^{r}$, sendo somente uma caso de carga necessário para o projeto. O comprimento $w$ é 300,00 mm e a altura em 60,00 mm considerando o tamanho do elemento triangular elemento em 3, $50 \mathrm{~mm}$, totalizando 5780 variáveis de projeto utilizando a formulação Lagragiana de ordem 1 (Alnæs et al., 2015) para cada elemento finito triangular. Na região $\partial \Omega_{\text {in }}^{r} / \partial \Omega_{\text {ou }}^{r}$ é aplicado um carregamento superficial de $0,0225 \mathrm{~N} / \mathrm{mm}^{2}$. As regiões $\partial \Omega_{0}^{r}$ e $\partial \Omega_{1}^{r}$ são relativas as condições de contorno de Dirichlet, onde $\partial \Omega_{0}^{r}$ é referente a fixação $(\mathbf{u}=\mathbf{0})$ e simetria no eixo $x\left(u_{0}=0\right)$ respetivamente.

As variáveis de projeto $\rho$ são inicializadas com o respetivo valor da restrição de fração volume imposta, com o valor de penalização do modelo de material SIMP em 3, 0 . Esta seleção de parâmetros foi baseado em diversos testes numéricos realizados.

Foram feitas comparações de resultados alterando a restrição de volume e por último modificando as restrições de incremento de carga.

Definido, portanto todas as condições e parâmetros relativos a solução do problema de equilíbrio via MEF, podemos agora definir o problema relativo a distribuição de 


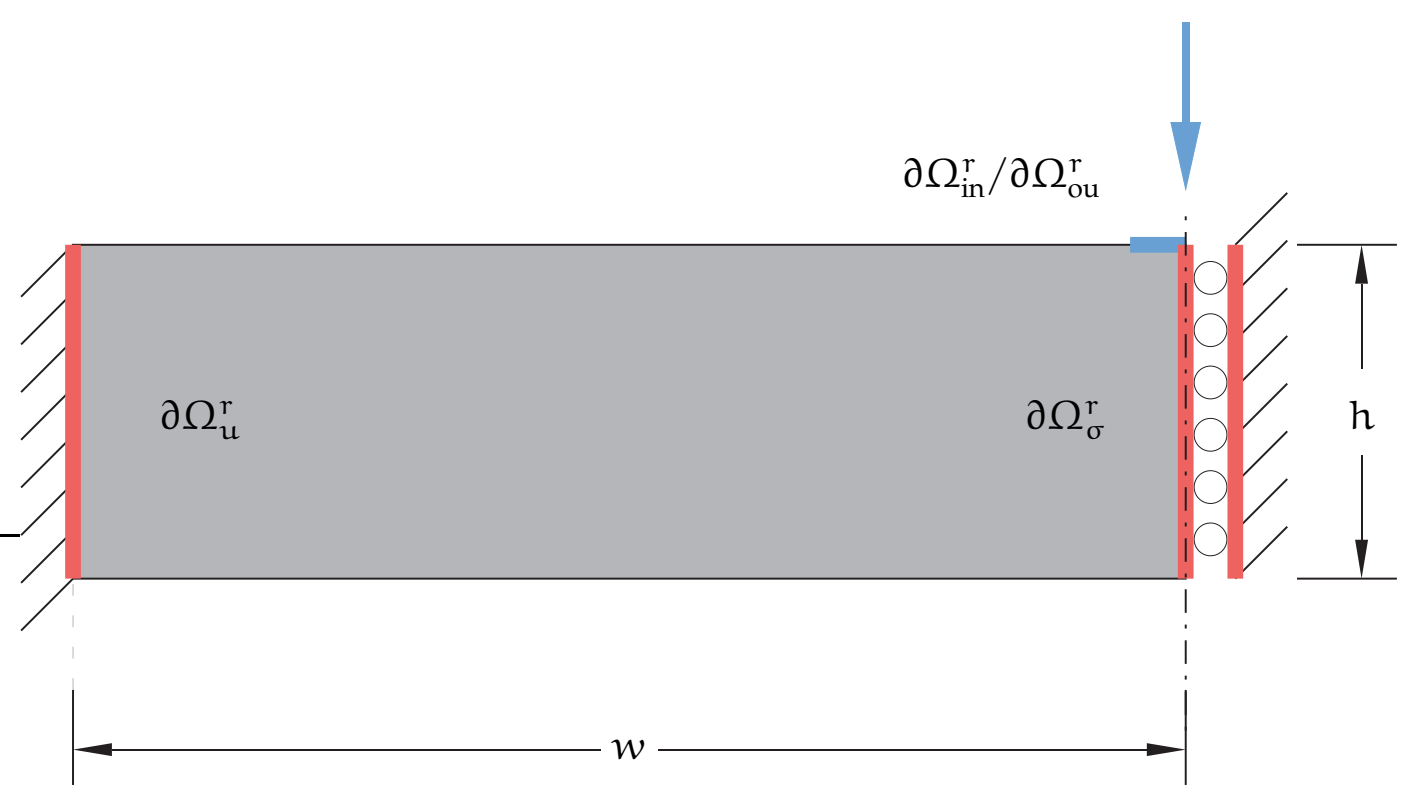

Figura 5.1: Configuração do domínio fixo estendido para à síntese de um mecanismo biestável.

material pelo MOT com a finalidade de projetar um mecanismo biestável:

$$
\begin{array}{ll}
\underset{\rho_{e} \in[0.0,1.0]}{\text { Min. }} & \Theta=\sum_{t}^{n_{t}(t)} \mathbf{f}_{\text {ext }} \cdot{ }^{(t)} \mathbf{u} \\
\text { Sujeito à } & \delta \Pi_{p}(\rho, u, \delta u)=0 \\
& { }^{\left(t_{\alpha}\right)} \bar{\lambda}=\{0,60 ; 0,20\} \\
& \bar{V}^{r}\left(\rho_{i}\right)-\underline{V} \leqslant 0,00 \\
& 2,5 m m=r_{\text {min }} \leqslant r^{*}\left(\Omega_{d}^{r}\right)
\end{array}
$$

onde o pseudo-tempo $\left(t_{\alpha}\right)$ selecionado para controlar as restrições de incremento de carga $\left({ }^{\left(t_{\alpha}\right)} \bar{\lambda}\right)$ são de 0,25 e 0,70 respectivamente. A função objetivo selecionada é refente a energia de deformação externa, os incrementos de pseudo-tempo selecionados foram $0,25,0,7$ e 1,0 .

\subsubsection{Restrição de Volume em 60\%}

Neste item são apresentados os resultados referente a restrição de volume em $60 \%$ do volume total. A topologia final da estrutura do mecanismo flexível biestável obtida pode ver verifica na figura 5.2. É uma topologia de fácil interpretação utilizando um 
ferramenta de CAD genérica.

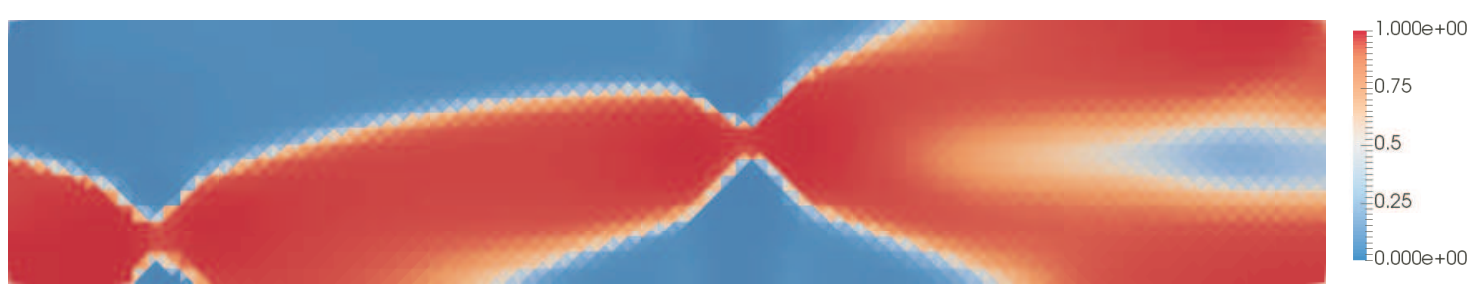

Figura 5.2: Topologia do mecanismo flexível biestável considerando a restrição de volume em $60 \%$.

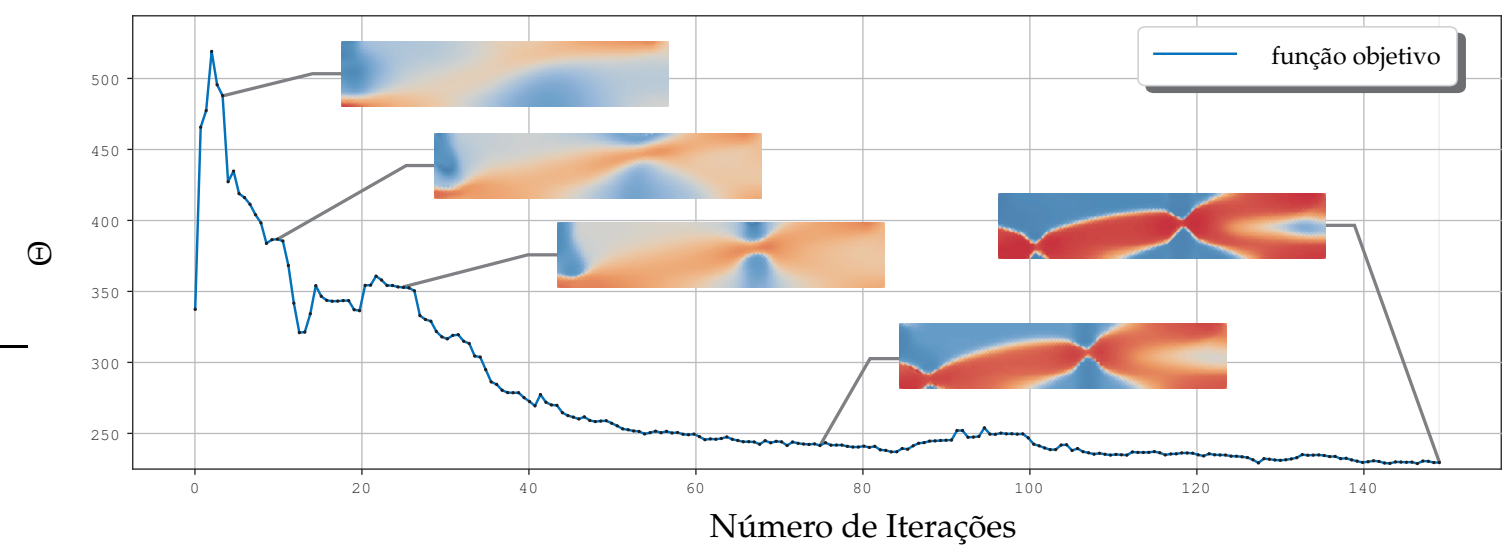

Figura 5.3: Evolução da função objetivo considerando a restrição de volume em $60 \%$.

Nas figuras 5.3 e 5.4 são apresentados gráficos referentes a função objetivo e de restrições referentes a convergência e evolução do problema. A evolução da topologia da estrutura são apontadas nas iterações 5, 15, 25, 50, 75 e 150 da figura 5.3.

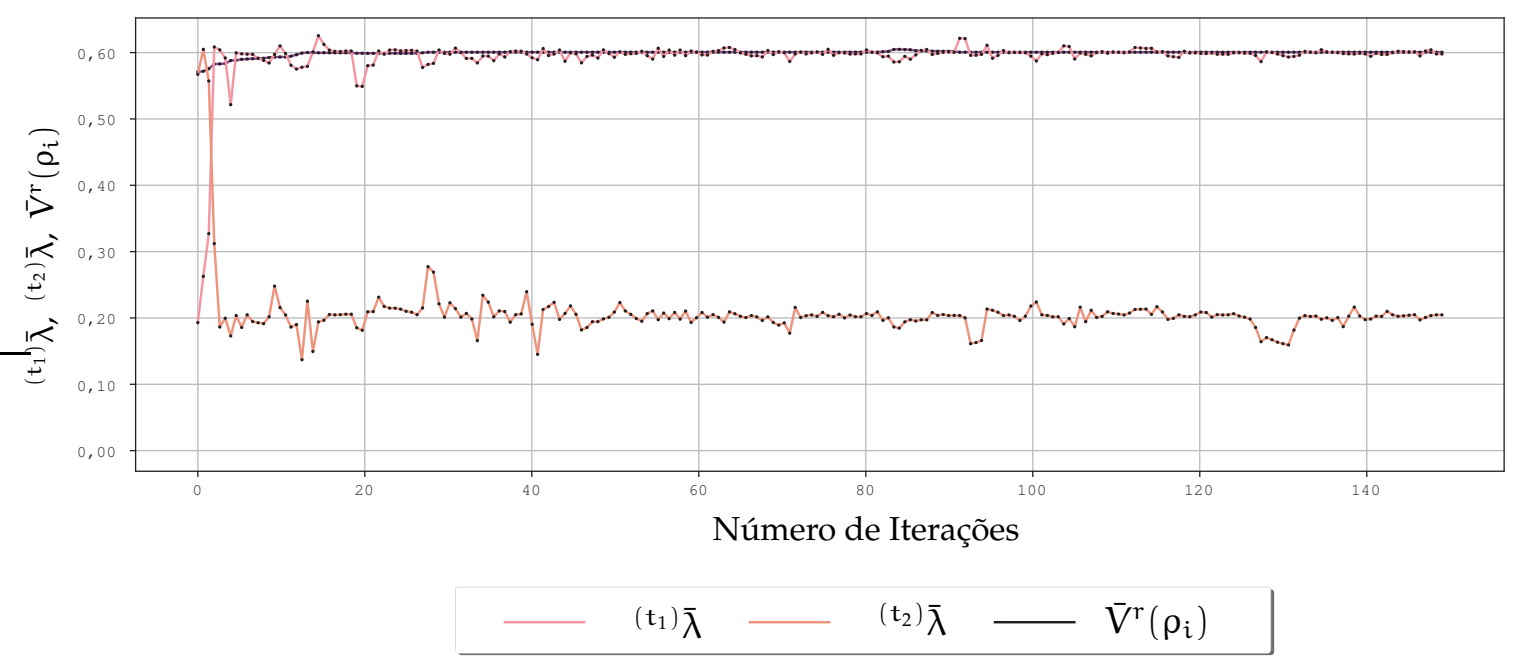

Figura 5.4: Gráfico de evolução das funções de restrições. 
Os gráficos referentes ao caminho de carga da estrutura em relação aos deslocamentos em $\partial \Omega_{\text {in }}^{r} / \partial \Omega_{\text {ou }}^{r}$ e pseudo tempo na região $\Omega_{\text {in }}^{r}$ são demonstrado pela figura 5.5, onde é possível verificar que as restrições de projeto não são totalmente respeitadas, mas os resultados estão muito próximos de satisfazer as restrições dentro dos limites impostos.

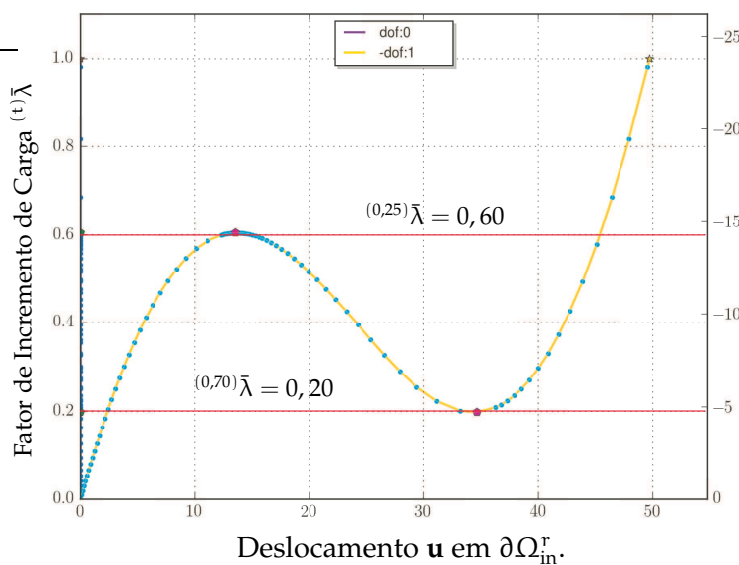

(a)

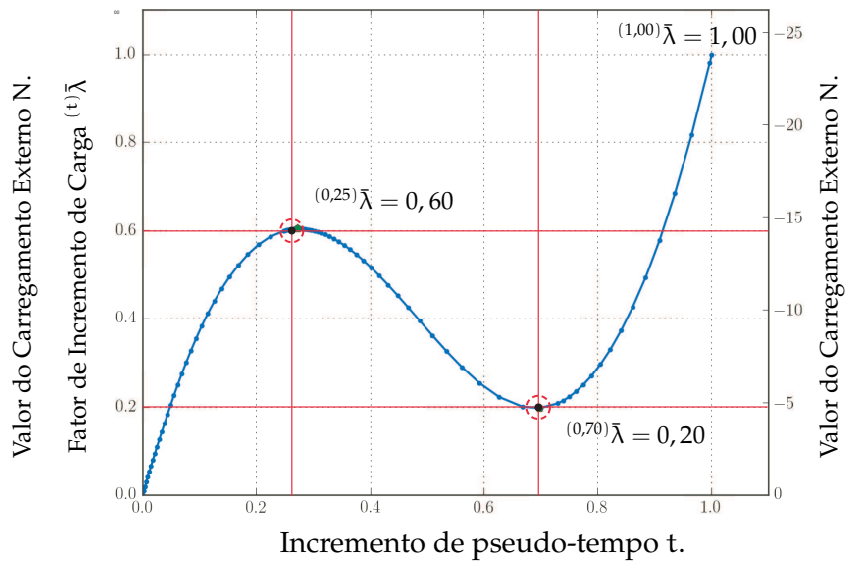

(b)

Figura 5.5: Gráfico de incremento de carga em relação ao deslocamento (a) e pseudo-tempo (b) considerando a restrição de volume em $60 \%$.

Foi feita uma interpretação da topologia final somente removendo elementos dentro de um valor de pseudo densidade que minimizasse o erro em relação ao modelo original. O modelo interpretado utilizando como função de erro da somatória das funções de restrições baseado no $\mathrm{MMQ}$ (5.2). O resultado da topologia é apresentado na figura 5.6.

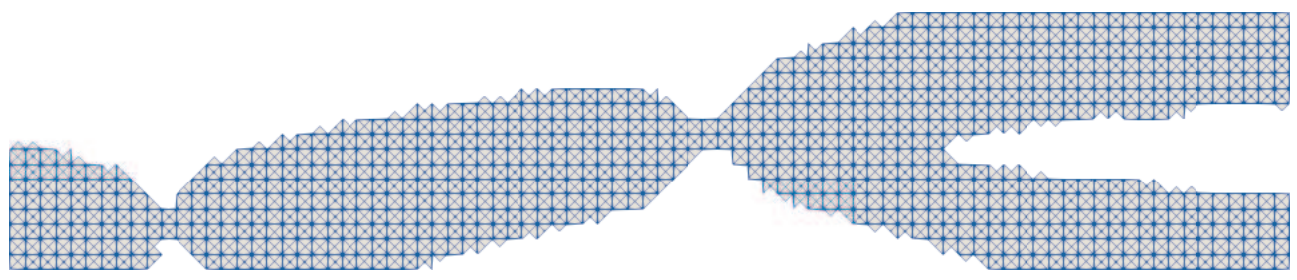

Figura 5.6: Malha da interpretação dos resultados considerando a restrição de volume em $60 \%$.

Foi feita a verificação do desempenho do mecanismo biestável em relação a interpretação obtida, os gráficos referentes ao caminho de carga da estrutura interpretada em relação aos deslocamentos e pseudo tempo na região $\Omega_{\text {in }}^{r}$ são demonstrados na figura 5.5, onde é possível verificar que a restrição não se mantém igual à restrição apresentada na figura 5.7 mesmo assim os resultados estão próximos de satisfazer as restrições de igualdade do problema referente ao fator de carga incremental. 


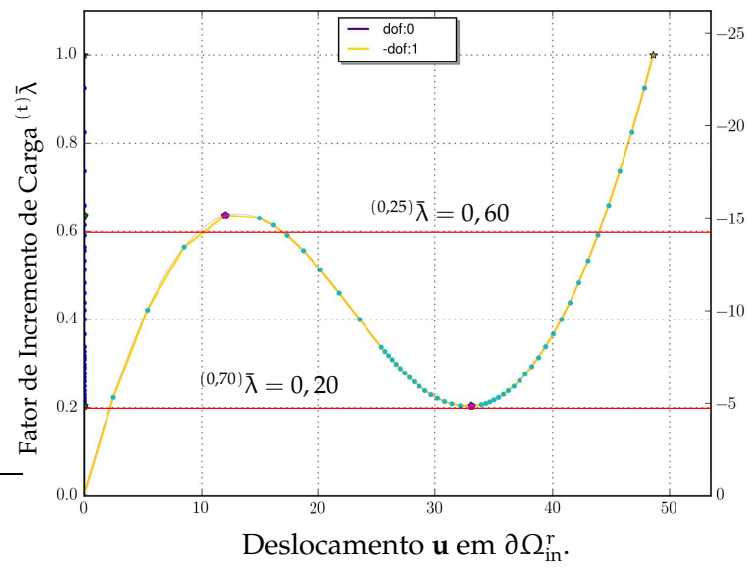

(a)
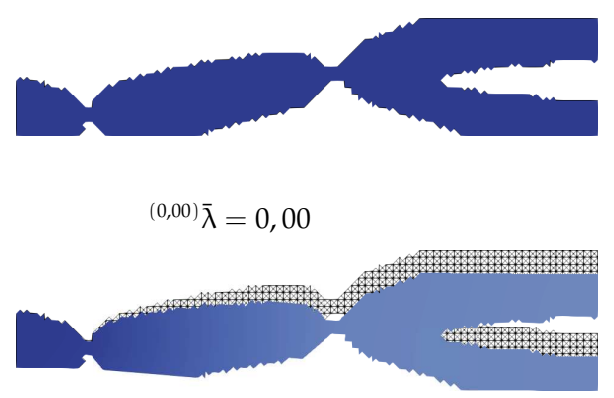

${ }^{(0,25)} \bar{\lambda}=0,60$

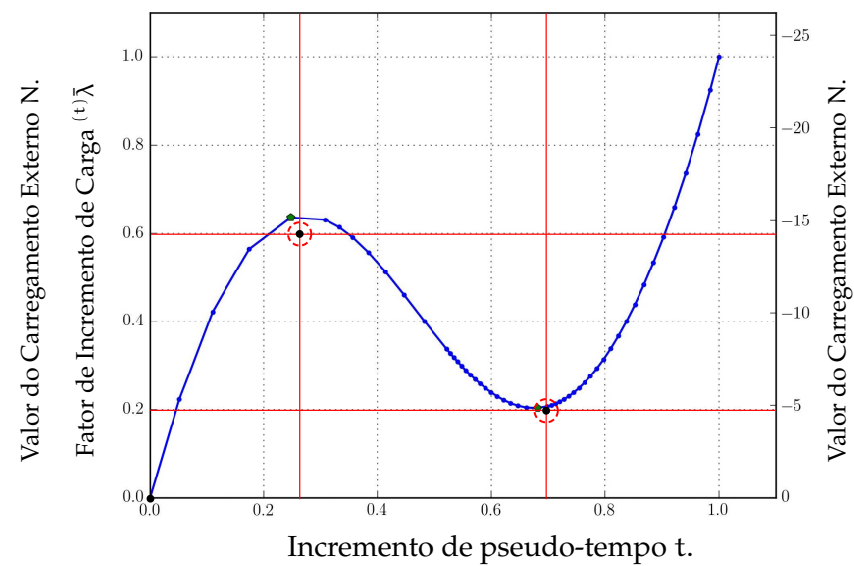

(b)

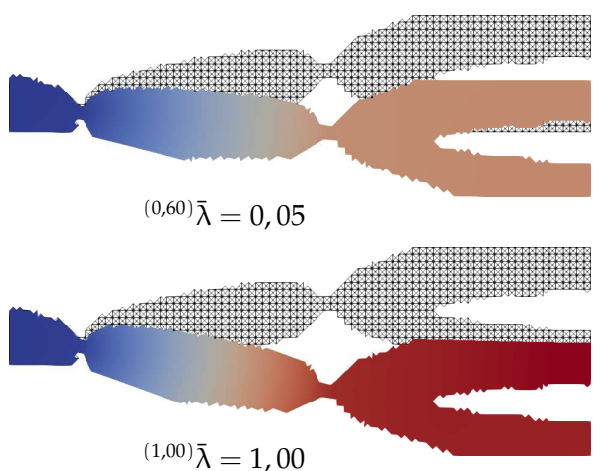

(c)

Figura 5.7: Gráfico de incremento de carga em relação ao deslocamento (a) e pseudo-tempo (b), configuração deformada (c) do modelo interpretado considerando a restrição de volume em $60 \%$.

\subsubsection{Restrição de Volume em 50\%}

Neste item são apresentados os resultados referente a restrição de volume em 50\% do volume total. A topologia final da estrutura do mecanismo flexível biestável obtida pode ver verifica na figura 5.8 .
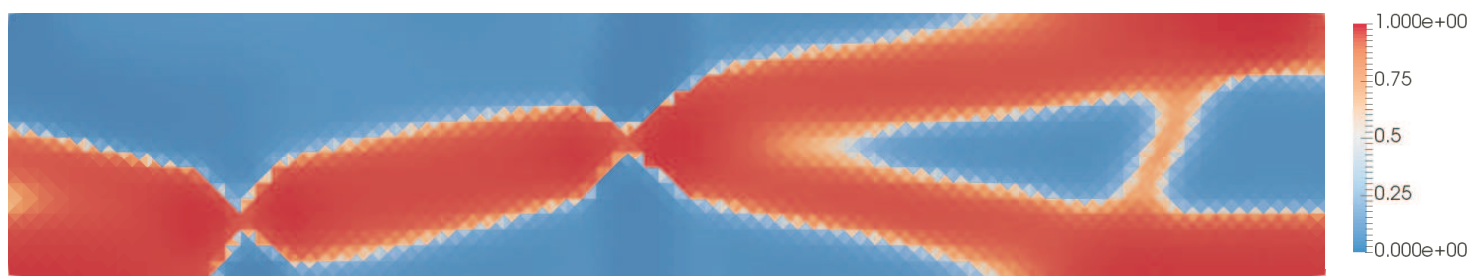

Figura 5.8: Topologia do mecanismo flexível biestável considerando a restrição de volume em 50\%.

Nas figuras 5.9 e 5.10 são apresentados gráficos referentes a função objetivo e de 
restrições referentes a convergência e evolução do problema. A evolução da topologia da estrutura são apontadas nas iterações 5, 15, 25, 50, 75 e 150.

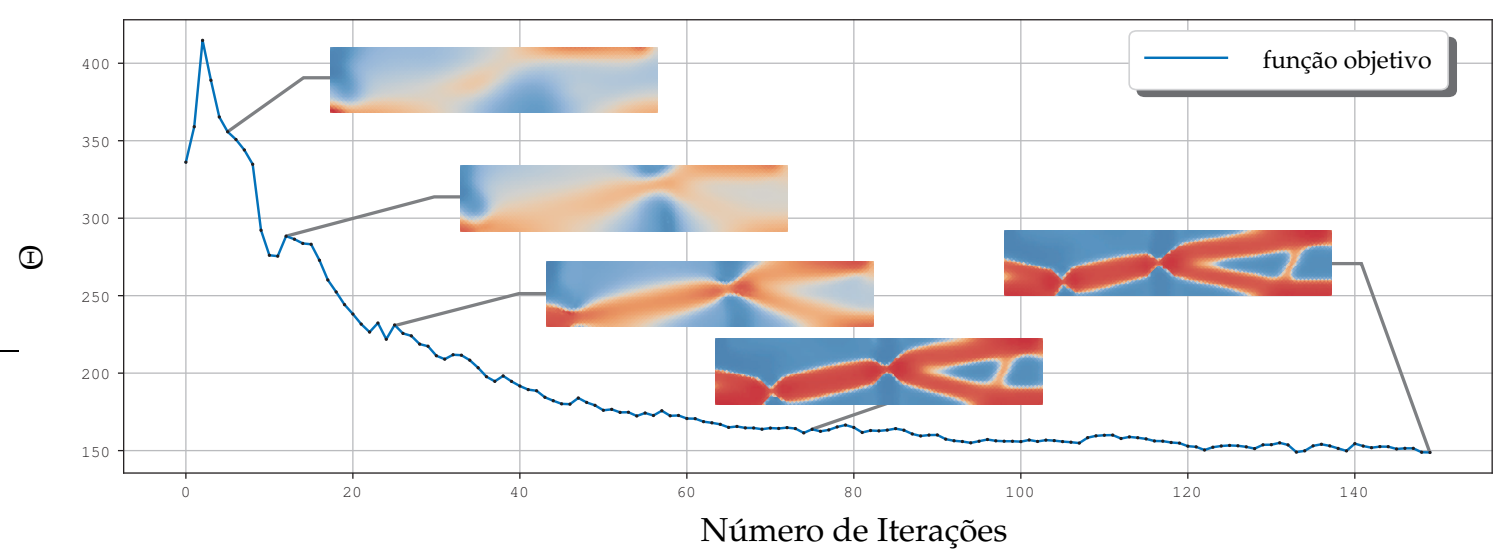

Figura 5.9: Evolução da função objetivo considerando a restrição de volume em $50 \%$.

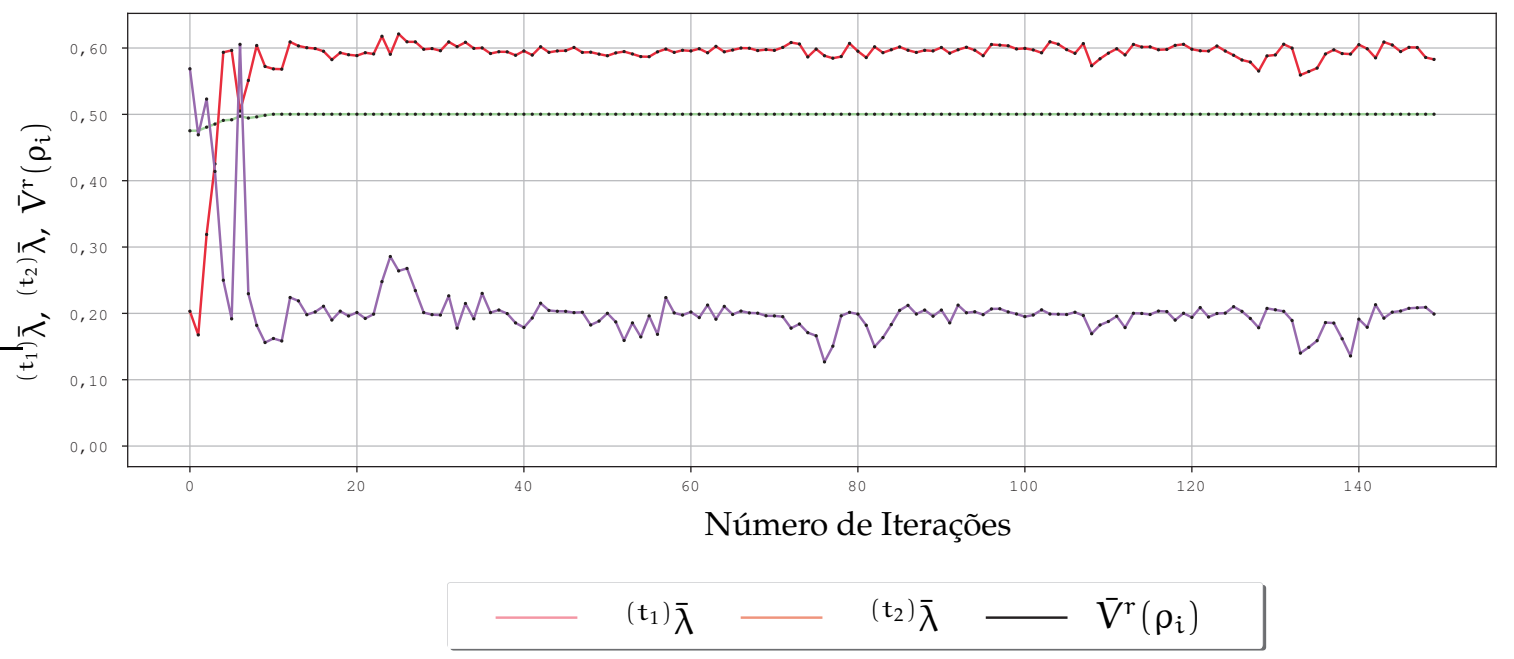

Figura 5.10: Gráfico de evolução das funções de restrições.

Os gráficos referentes ao caminho de carga da estrutura em relação aos deslocamentos em $\partial \Omega_{\text {in }}^{r} / \partial \Omega_{\text {ou }}^{r}$ e pseudo tempo na região $\Omega_{\text {in }}^{r}$ são demonstrados pela figura 5.11 , onde é possível verificar que as restrições de projeto não são totalmente respeitadas, mas os resultados estão muito próximos de satisfazer as restrições dentro dos limites impostos.

Foi feita uma interpretação da topologia final somente removendo elementos dentro de um valor de pseudo densidade que minimizasse o erro entre o modelo original. O modelo interpretado utilizando como função de erro em relação das funções de restrições. O resultado da topologia é apresentado na figura 5.12 . 


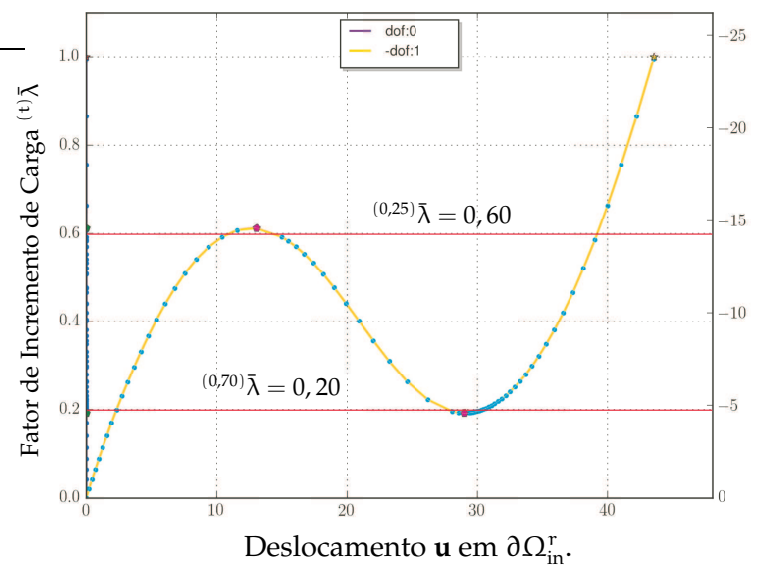

(a)

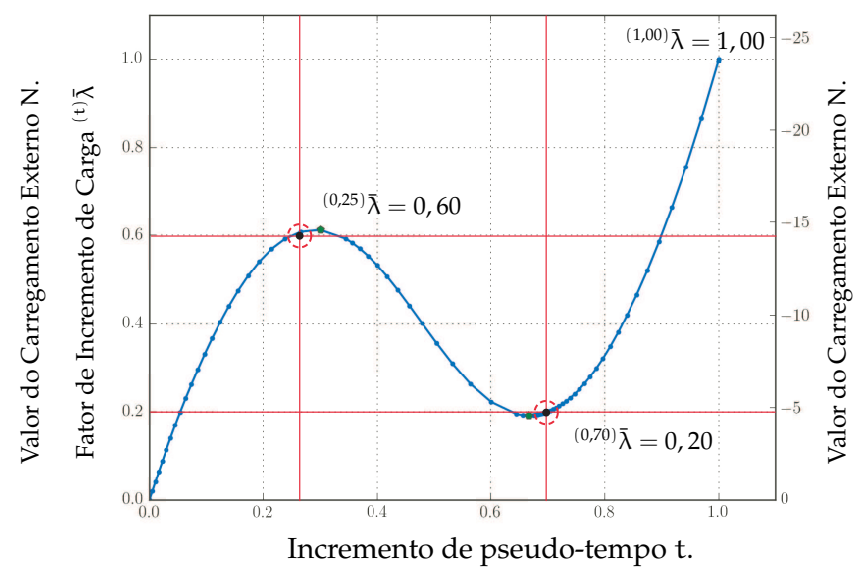

(b)

Figura 5.11: Gráfico de incremento de carga em relação ao deslocamento (a) e pseudo-tempo (b) considerando a restrição de volume em $50 \%$.

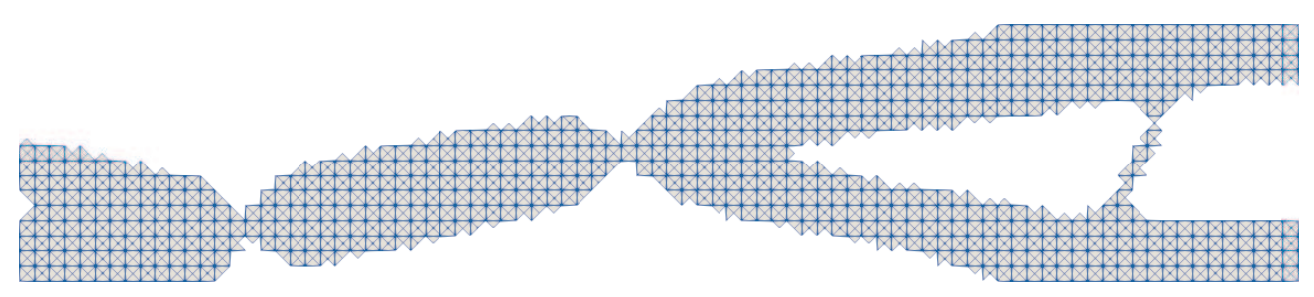

Figura 5.12: Malha da interpretação dos resultados considerando a restrição de volume em $50 \%$.

Foi feita a verificação do desempenho do mecanismo biestável em relação à interpretação obtida, os gráficos referentes ao caminho de carga da estrutura interpretada em relação aos deslocamentos e pseudo tempo na região $\Omega_{\text {in }}^{r}$ são demonstrados na figura 5.11 , onde é possível verificar que a restrição não se mantém igual à restrição apresentada na figura 5.13. Mesmo assim a restrição em ${ }^{\left(t_{1}\right)} \bar{\lambda}=0,6$ está muito próxima do valor desejado, no entanto em ${ }^{\left(t_{1}\right)} \bar{\lambda}=0,4$ a restrição se encontra um pouco fora do pseudo-tempo desejado, mas muito próximo do fator de incremento de carga selecionado.

\subsubsection{Restrição de Volume em 40\%}

Neste item são apresentados os resultados referente a restrição de volume em 40\% do volume total. A topologia final da estrutura do mecanismo flexível biestável obtida pode ver verifica na figura 5.14 .

Nas figuras 5.9 e 5.10 são apresentados gráficos referentes a função objetivo e de 


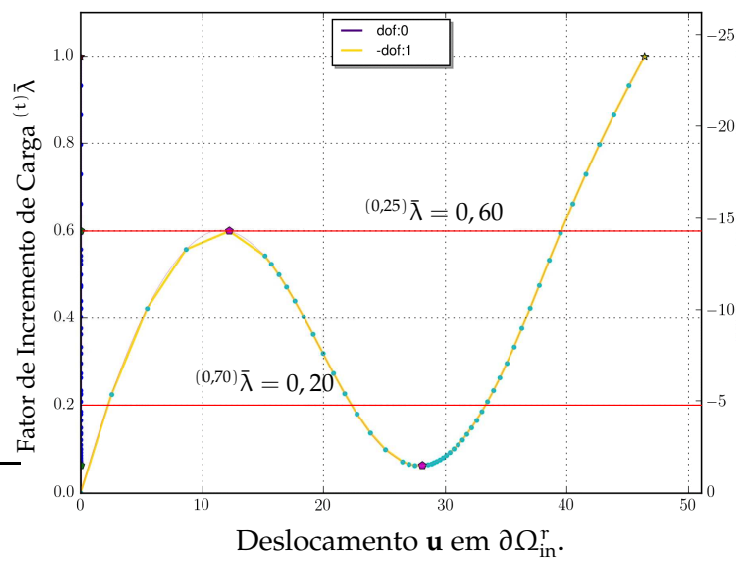

(a)

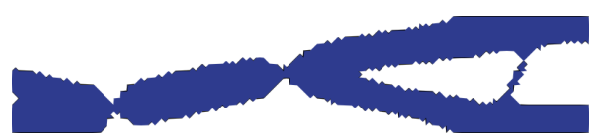

${ }^{(0,00)} \bar{\lambda}=0,00$

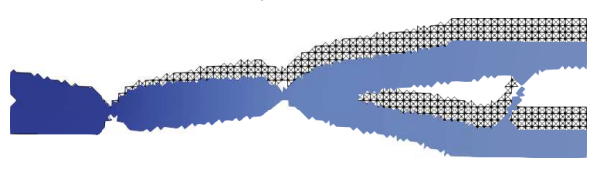

${ }^{(0,25)} \bar{\lambda}=0,60$

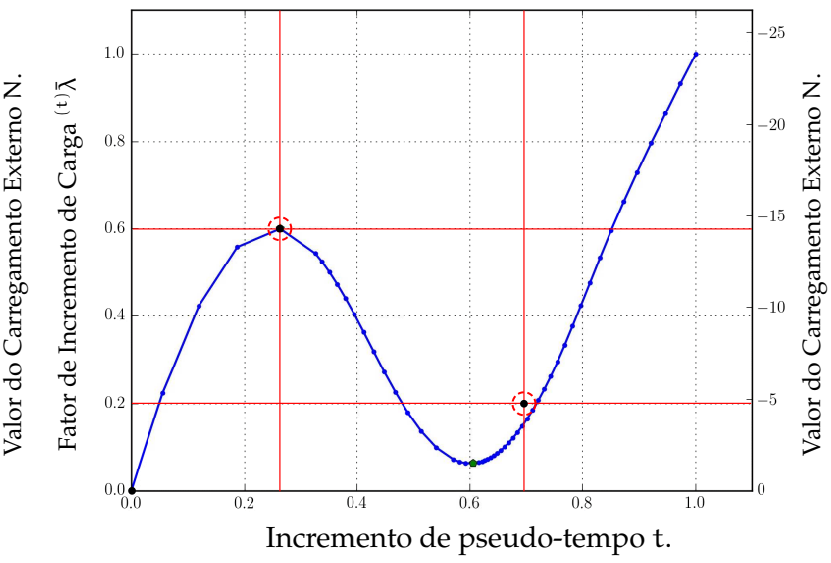

(b)

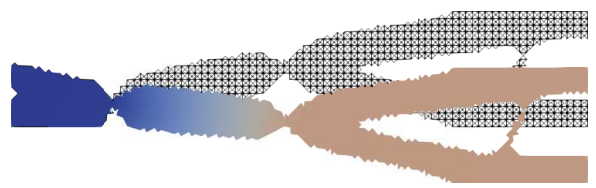

${ }^{(0,60)} \bar{\lambda}=0,05$

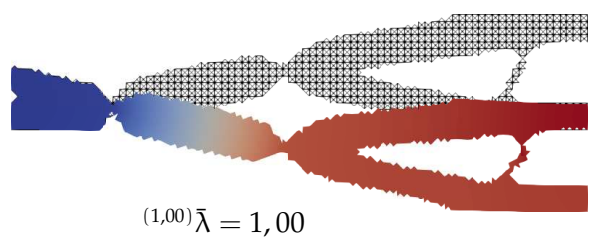

${ }^{(1,00)} \bar{\lambda}=1,00$

(c)

Figura 5.13: Gráfico de incremento de carga em relação ao deslocamento (a) e pseudo-tempo (b), configuração deformada (c) do modelo interpretado considerando a restrição de volume em $50 \%$.
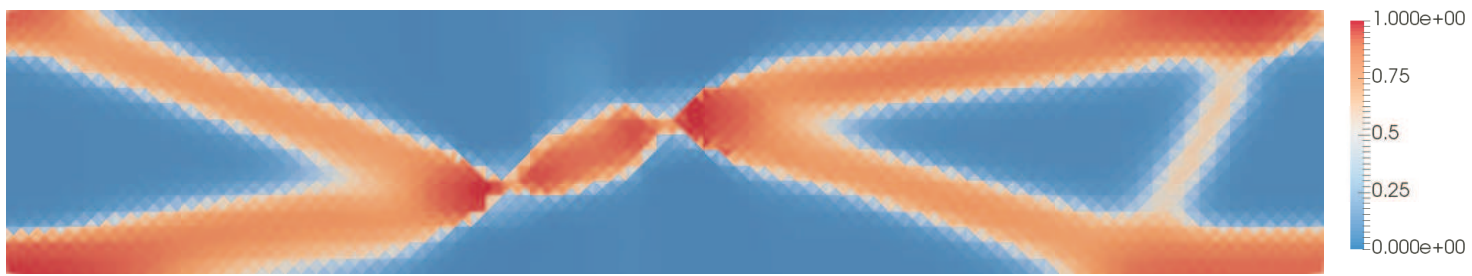

Figura 5.14: Topologia do mecanismo flexível biestável considerando a restrição de volume em $40 \%$.

restrições referentes a convergência e evolução do problema. A evolução da topologia da estrutura são apontadas nas iterações 10, 25, 50, 100 e 150.

Os gráficos referentes ao caminho de carga da estrutura em relação aos deslocamentos em $\partial \Omega_{\text {in }}^{r} / \partial \Omega_{\text {ou }}^{r}$ e pseudo tempo na região $\Omega_{\text {in }}^{r}$ são demonstrados na figura 5.17, onde é possível verificar que as restrições de projeto estão próximas de serem respeitadas, no entanto a curva não está dentro dos limites de carga ${ }^{\left(\mathrm{t}_{1}\right)} \bar{\lambda}=0,6 \mathrm{e}^{\left(\mathrm{t}_{2}\right)} \bar{\lambda}=0,2$ como desejado. 


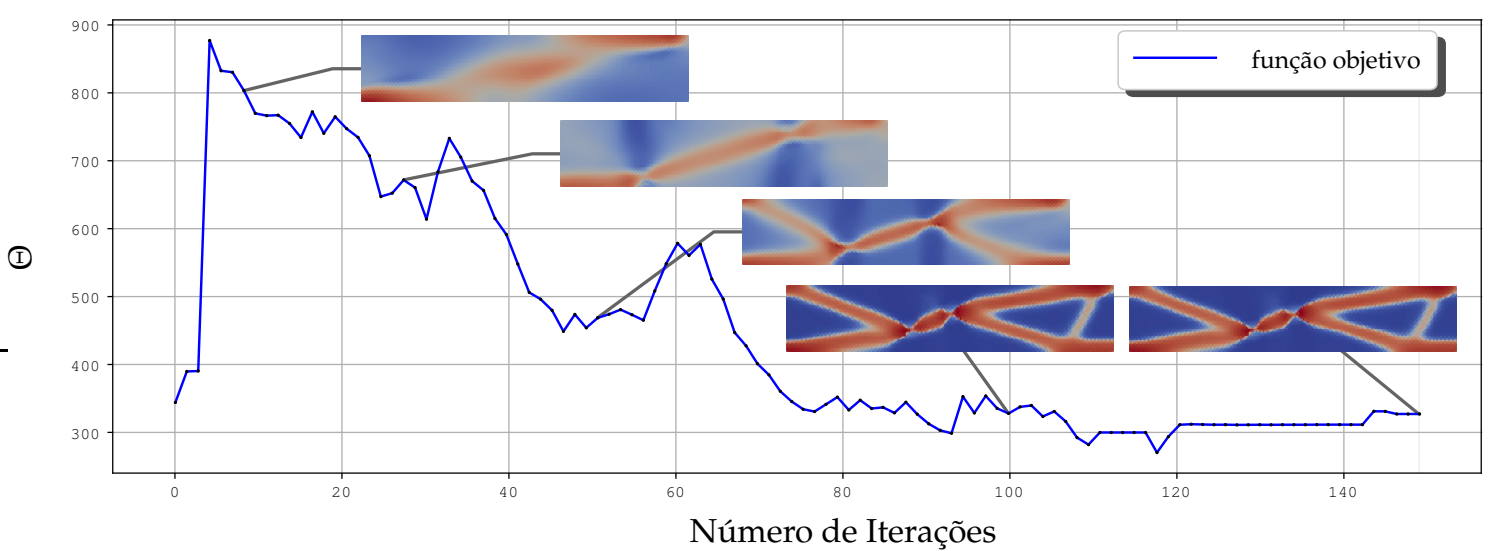

Figura 5.15: Evolução da função objetivo considerando a restrição de volume em $40 \%$.

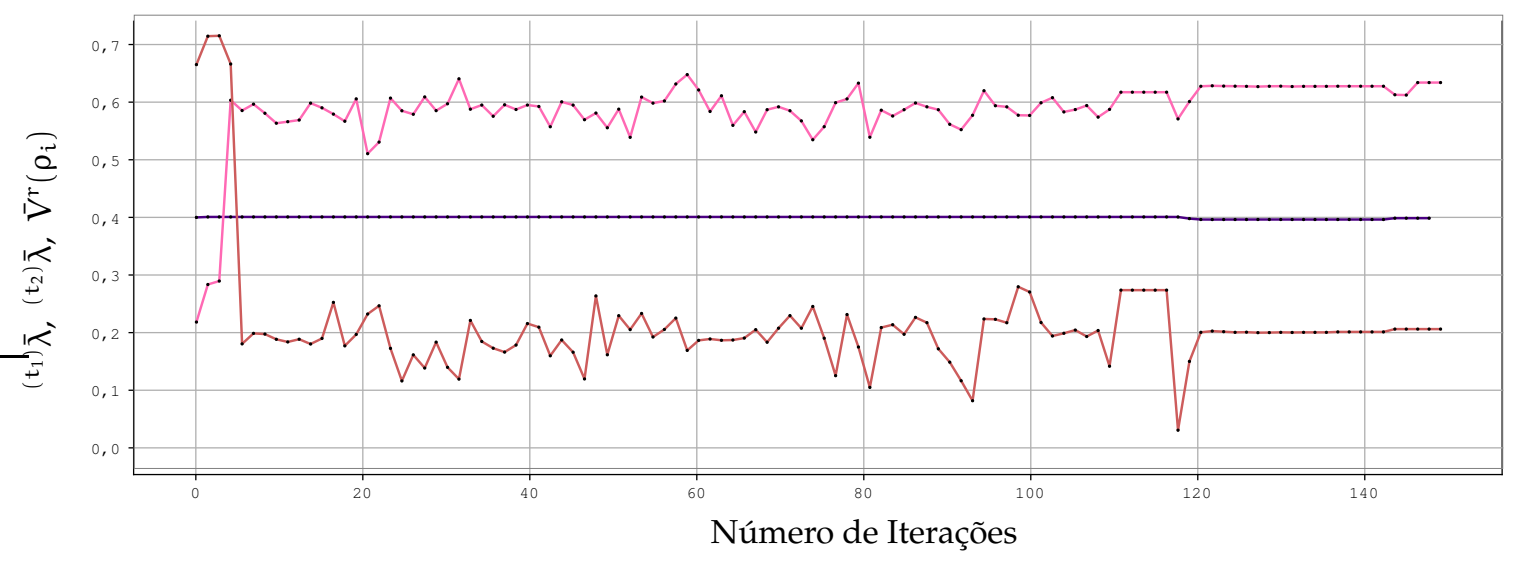

${ }^{\left(t_{1}\right)} \bar{\lambda} \quad{ }^{\left(t_{2}\right)} \bar{\lambda} \quad \longrightarrow \quad \bar{V}^{\mathrm{r}}\left(\rho_{\mathrm{i}}\right)$

Figura 5.16: Gráfico de evolução das funções de restrições.

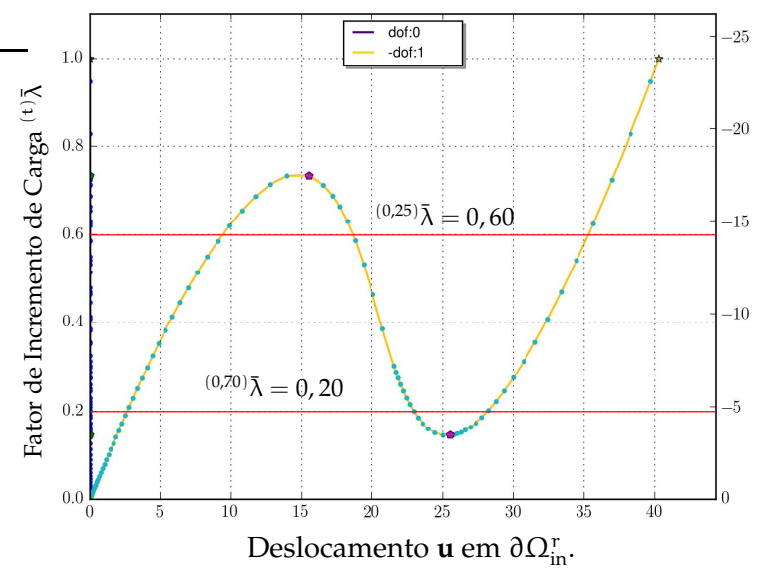

(a)

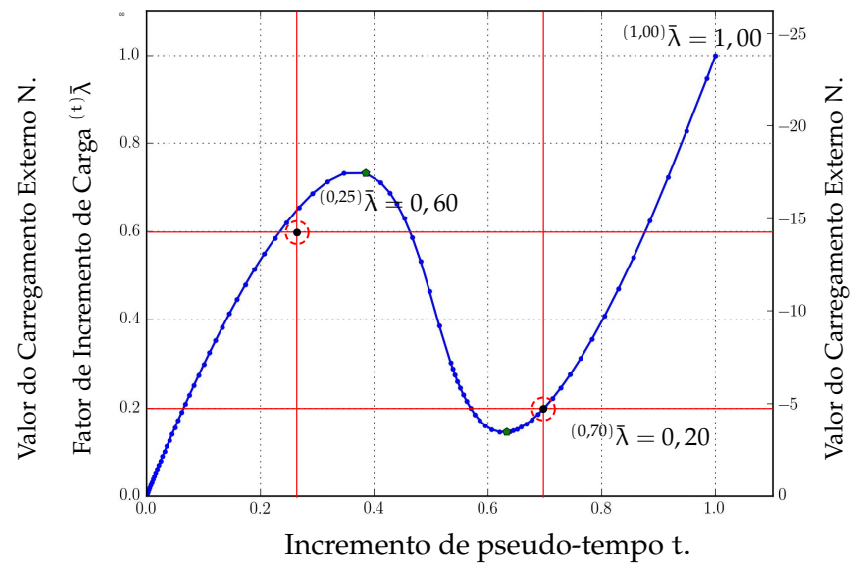

(b)

Figura 5.17: Gráfico de incremento de carga em relação ao deslocamento (a) e pseudo-tempo (b) considerando a restrição de volume em $40 \%$. 
Foi feita uma interpretação da topologia final somente removendo elementos dentro de um valor de pseudo densidade que minimizasse o erro em relação modelo original. $O$ modelo interpretado utiliza como função de erro a somatória das funções de restrições. O resultado da topologia é apresentado na figura 5.18 .

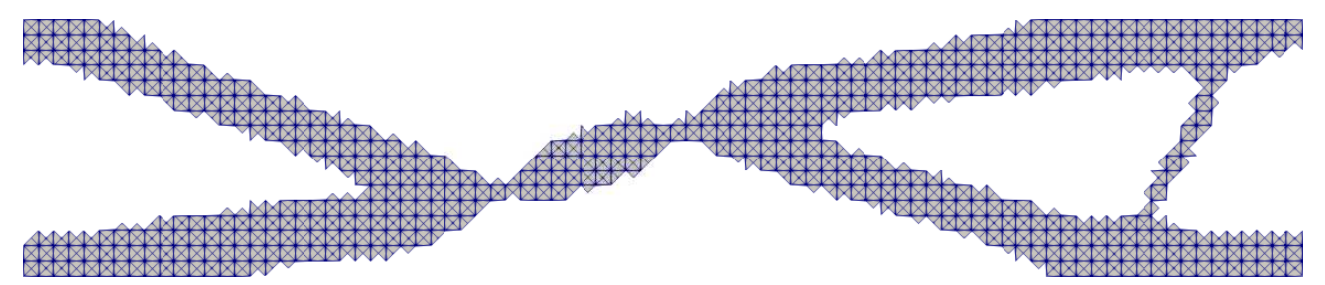

Figura 5.18: Malha da interpretação dos resultados considerando a restrição de volume em $40 \%$.

Foi feita a verificação da performance do mecanismo biestável em relação à interpretação obtida, os gráficos referentes ao caminho de carga da estrutura interpretada em relação aos deslocamentos e pseudo tempo na região $\Omega_{\text {in }}^{r}$ é demonstrado na figura 5.17, onde é possível verificar que a restrição não se mantém igual à restrição imposta.

\subsubsection{Restrição de Volume em 50\% com alteração na restrição de carga}

Neste item são apresentados os resultados referente a restrição de volume em 50\% do volume total, no entanto alterando as restrições de incremento de carga em $t_{1}$ e $t_{2}$ conforme equação (5.3). A topologia final da estrutura do mecanismo flexível biestável obtida pode ser verificada na figura 5.20 .

$$
\begin{aligned}
& { }^{(0,25)} \bar{\lambda}=0,40 \\
& { }^{(0,60)} \bar{\lambda}=0,05
\end{aligned}
$$

Nas figuras 5.21 e 5.22 são apresentados gráficos referentes a função objetivo e de restrições referentes a convergência e evolução do problema. A evolução da topologia da estrutura são apontadas nas iterações 5, 25, 50, 75, 100 e 150.

Nas figuras 5.21 e 5.22 são apresentados gráficos referentes a função objetivo e de 


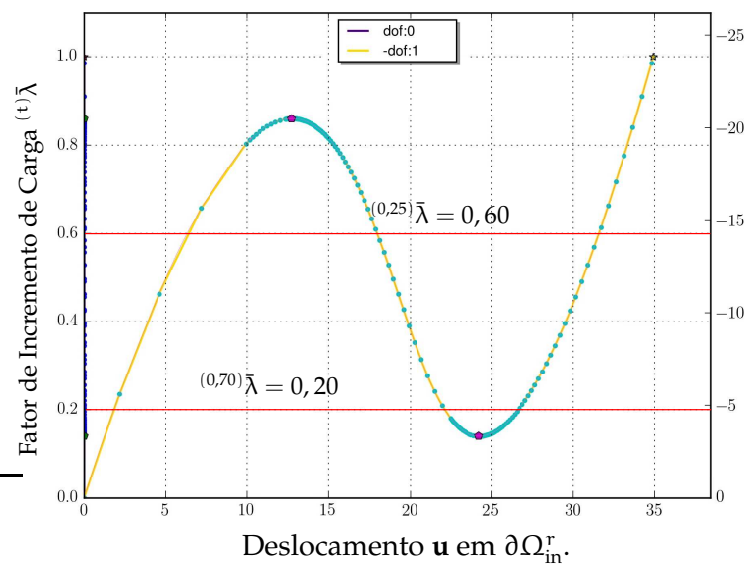

(a)

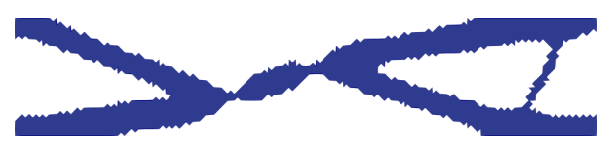

${ }^{(0,00)} \bar{\lambda}=0,00$



${ }^{(0,25)} \bar{\lambda}=0,60$

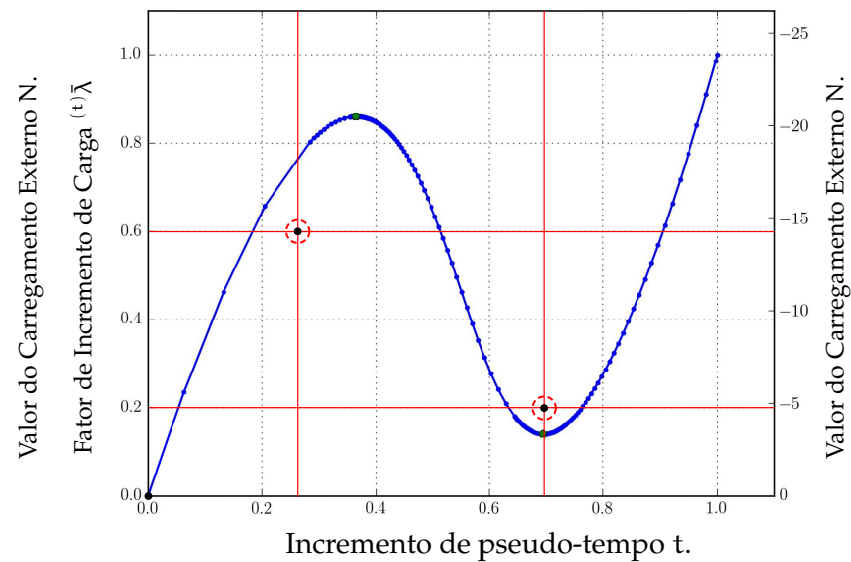

(b)

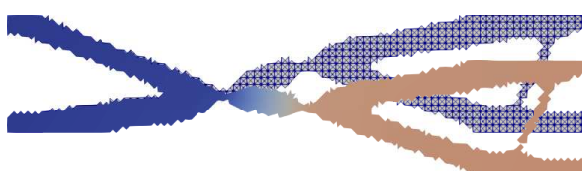

${ }^{(0,60)} \bar{\lambda}=0,05$

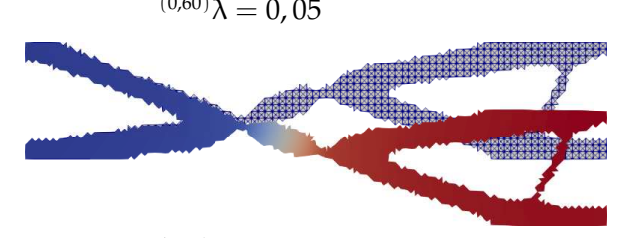

${ }^{(1,00)} \bar{\lambda}=1,00$

(c)

Figura 5.19: Gráfico de incremento de carga em relação ao deslocamento (a) e pseudo-tempo (b), configuração deformada (c) do modelo interpretado considerando a restrição de volume em $40 \%$.
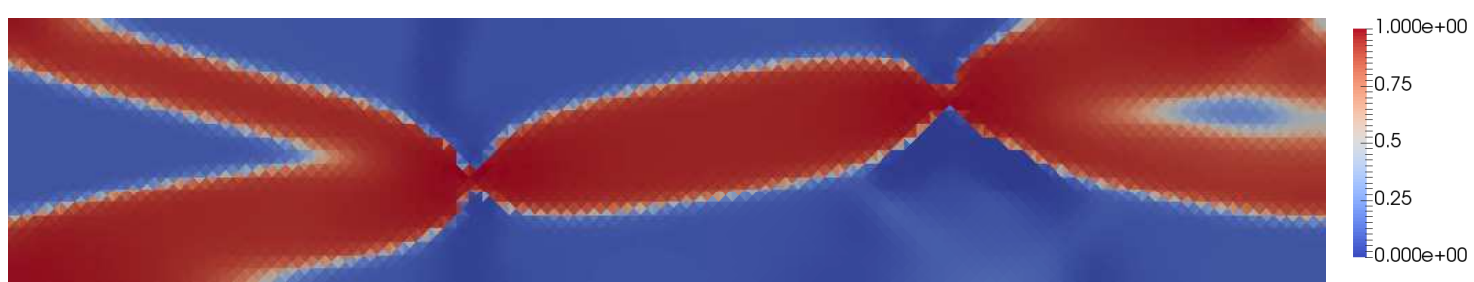

Figura 5.20: Topologia do mecanismo flexível biestável considerando a restrição de volume em 50\%.

restrições referentes a convergência e evolução do problema. A evolução da topologia da estrutura são apontadas nas iterações 5, 25, 50, 75, 100 e 150.

Os gráficos referentes ao caminho de carga da estrutura em relação aos deslocamentos em $\partial \Omega_{\text {in }}^{r} / \partial \Omega_{\mathrm{ou}}^{r}$ e pseudo tempo na região $\Omega_{\text {in }}^{r}$ são demonstrados pela figura 5.23 , onde é possível verificar que as restrições de projeto não são totalmente respeitadas, mas os resultados estão muito próximos de satisfazer as restrições dentro dos limites impostos. 


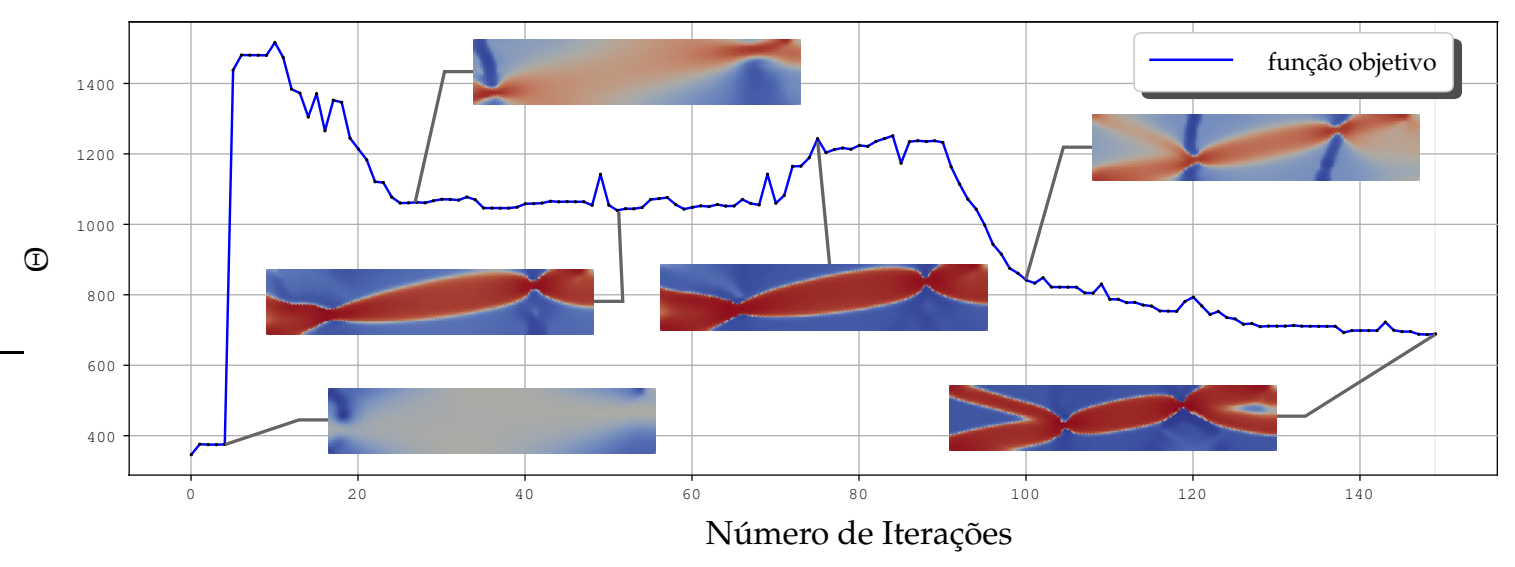

Figura 5.21: Evolução da função objetivo considerando a restrição de volume em $50 \%$.

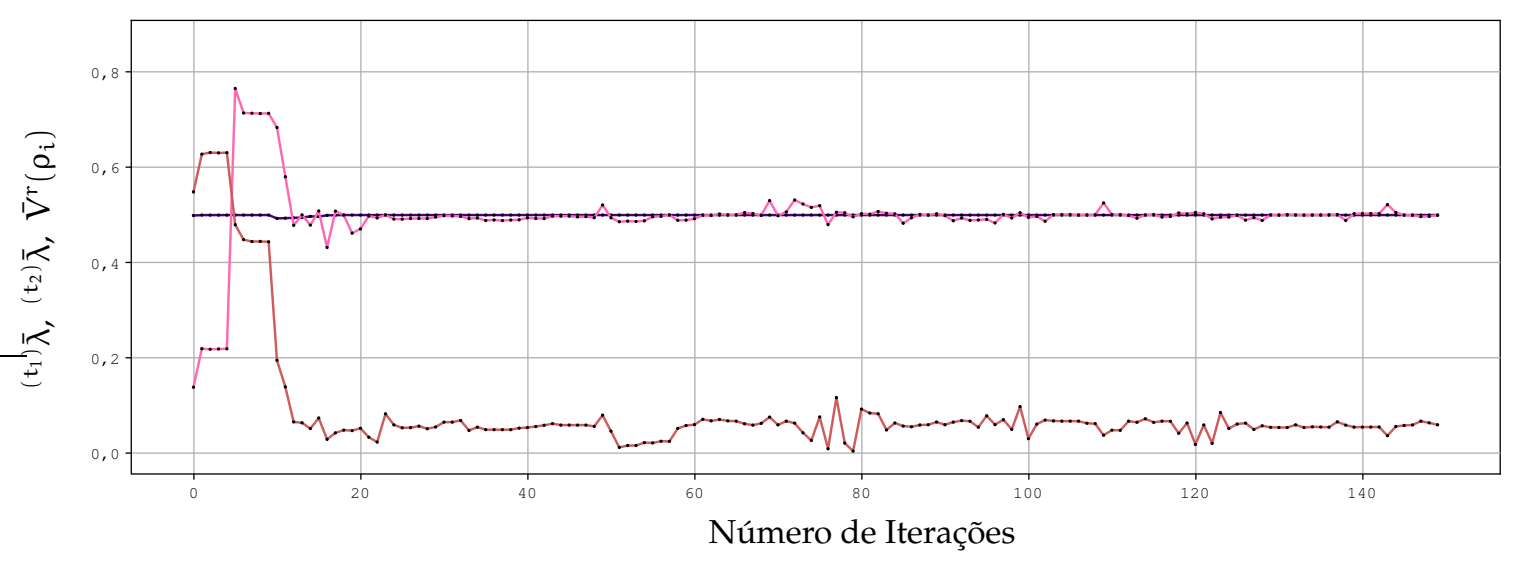

$$
\longrightarrow{ }^{\left(t_{1}\right)} \bar{\lambda} \longrightarrow{ }^{\left(t_{2}\right)} \bar{\lambda} \quad \bar{V}^{r}\left(\rho_{i}\right)
$$

Figura 5.22: Gráfico de evolução das funções de restrições.

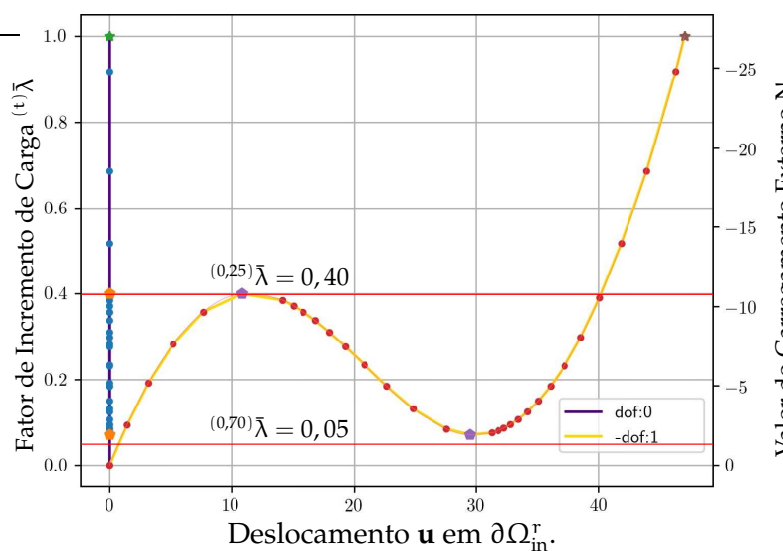

(a)

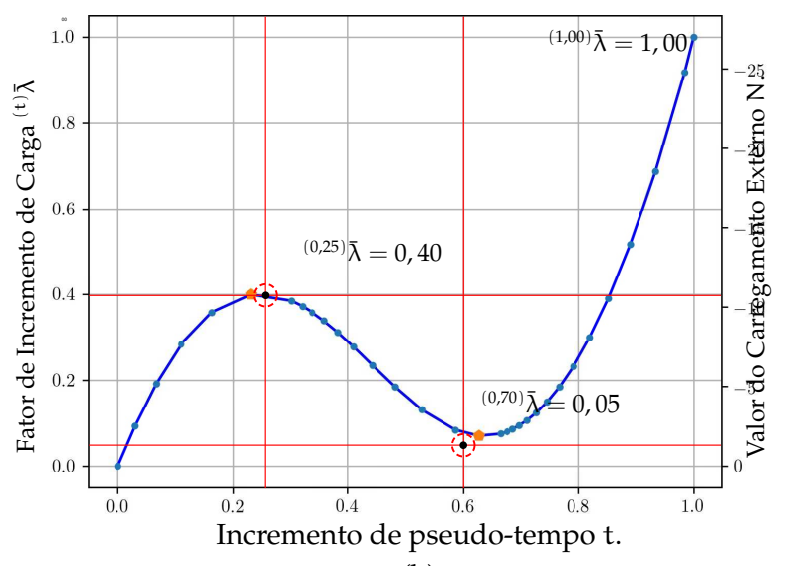

(b)

Figura 5.23: Gráfico de incremento de carga em relação ao deslocamento (a) e pseudo-tempo (b) considerando a restrição de volume em $50 \%$. 


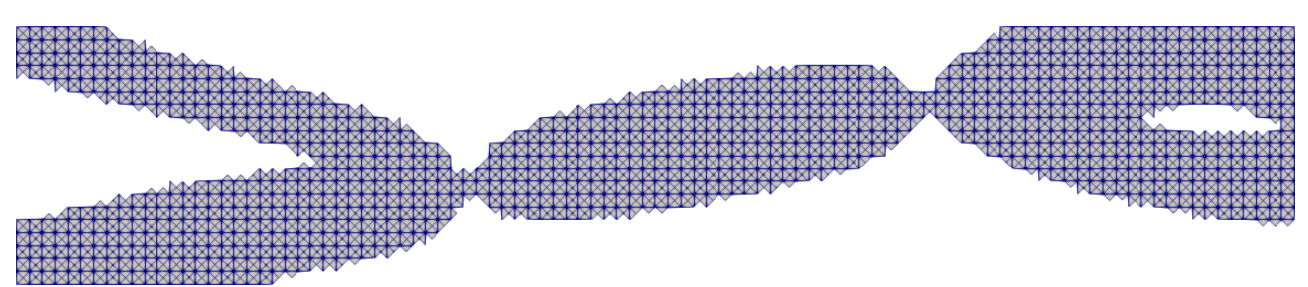

Figura 5.24: Malha da interpretação dos resultados considerando a restrição de volume em $50 \%$.

Uma verificação do desempenho do mecanismo biestável em relação a interpretação foi feita e os gráficos referentes ao caminho de carga da estrutura interpretada em relação aos deslocamentos e pseudo tempo na região $\Omega_{\text {in }}^{r}$ são apresentados na figura 5.23, onde é possível verificar que a restrição se mantém muito próxima à restrição apresentada na figura 5.25 ,

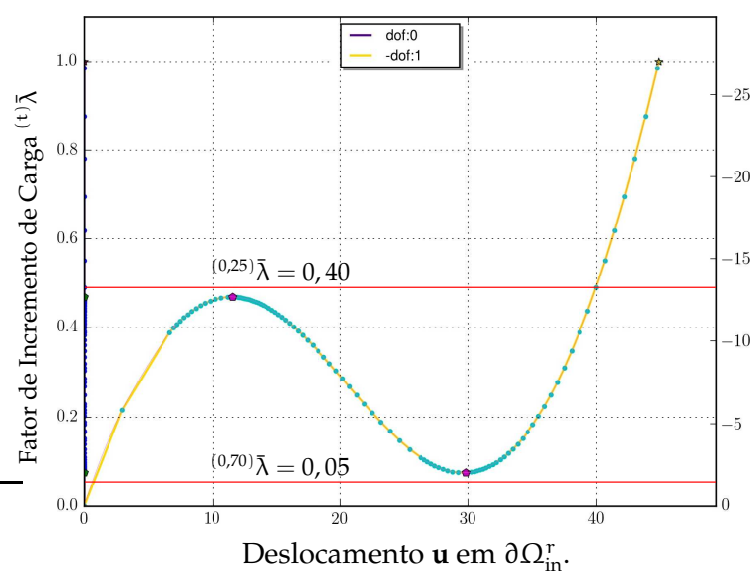

(a)
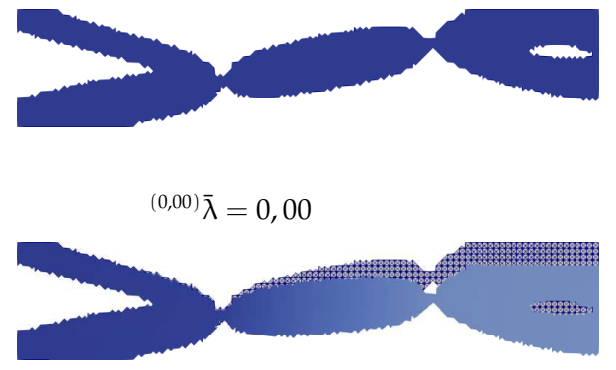

${ }^{(0,25)} \bar{\lambda}=0,40$

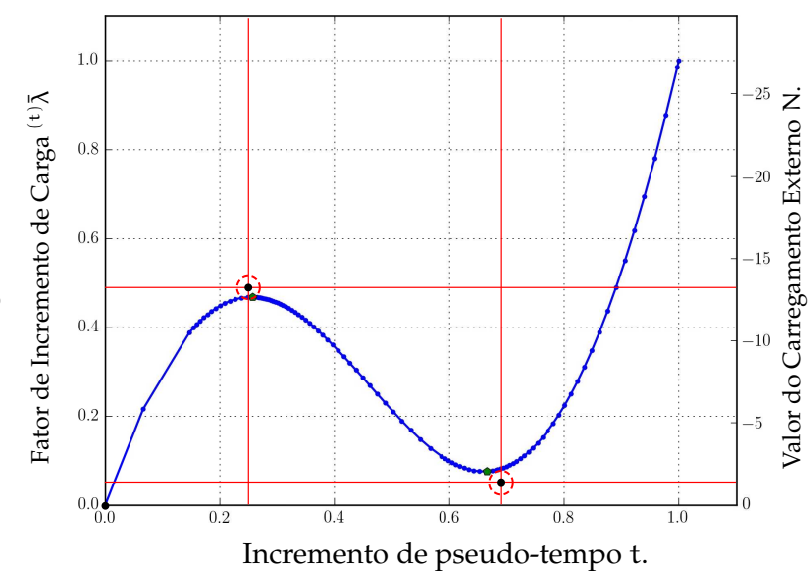

(b)

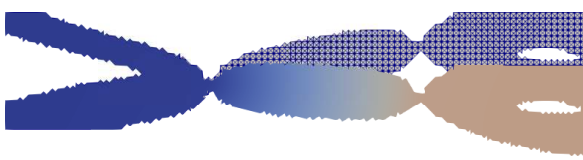

Desloc. Mag. mm

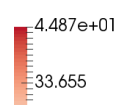

${ }^{(0,70)} \bar{\lambda}=0,05$

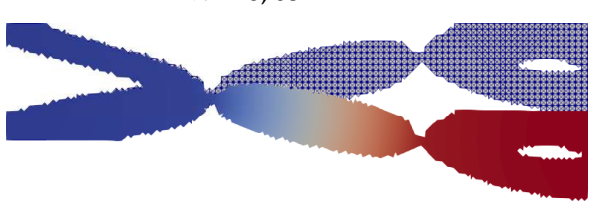

${ }^{(1,00)} \bar{\lambda}=1,00$

(c)

Figura 5.25: Gráfico de incremento de carga em relação ao deslocamento (a) e pseudo-tempo (b), configuração deformada (c) do modelo interpretado considerando a restrição de volume em 50\%.

É importante relatar que a topologia obtida entre as iterações 25 à 50 são muito semelhantes as topologias apresentadas na literatura pelos trabalhos de Bruns et al. 
(2002b); James e Waisman (2016)

\subsection{Mecanismo Inversor}

Nesta seção, é apresentada a formulação de mecanismos flexíveis inversor que utiliza o mecanismo de flambagem não linear a porta de entrada $\partial \Omega_{\text {in }}^{r}$ desenvolvida neste trabalho. Busca-se uma estrutura que possua duas posições de equilíbrio estáveis $\left[0 ; t_{1}\right]$, $\left[t_{2} ; 1,0\right]$ em $\partial \Omega_{\text {in }}^{r}$ separados por uma região de equilíbrio instável $\left\{t_{1} ; t_{2}\right\}$, onde no tempo $t_{2}$ o deslocamento em $\partial \Omega_{\text {ou }}^{r}$ sem carregamento.

Na figura 5.26 são apresentadas as condições de contorno $\partial \Omega_{\mathfrak{u}}^{r}$ e $\partial \Omega_{\sigma}^{r}$, sendo necessários dois casos de carga para o projeto. O primeiro caso referente ao carregamento na porta de entrada $\partial \Omega_{\mathrm{in}}^{\mathrm{r}}$ e o segundo referente ao carregamento na porta de saída $\partial \Omega_{\mathrm{ou}}^{\mathrm{r}}$. O comprimento $w$ é 300, 0 mm e a altura é igual a 150, 0 mm considerando o tamanho de elemento em 6,0 mm, totalizando 7200 variáveis de projeto. A formulação Lagragiana de ordem 1 foi utilizada para cada elemento finito triangular (?).

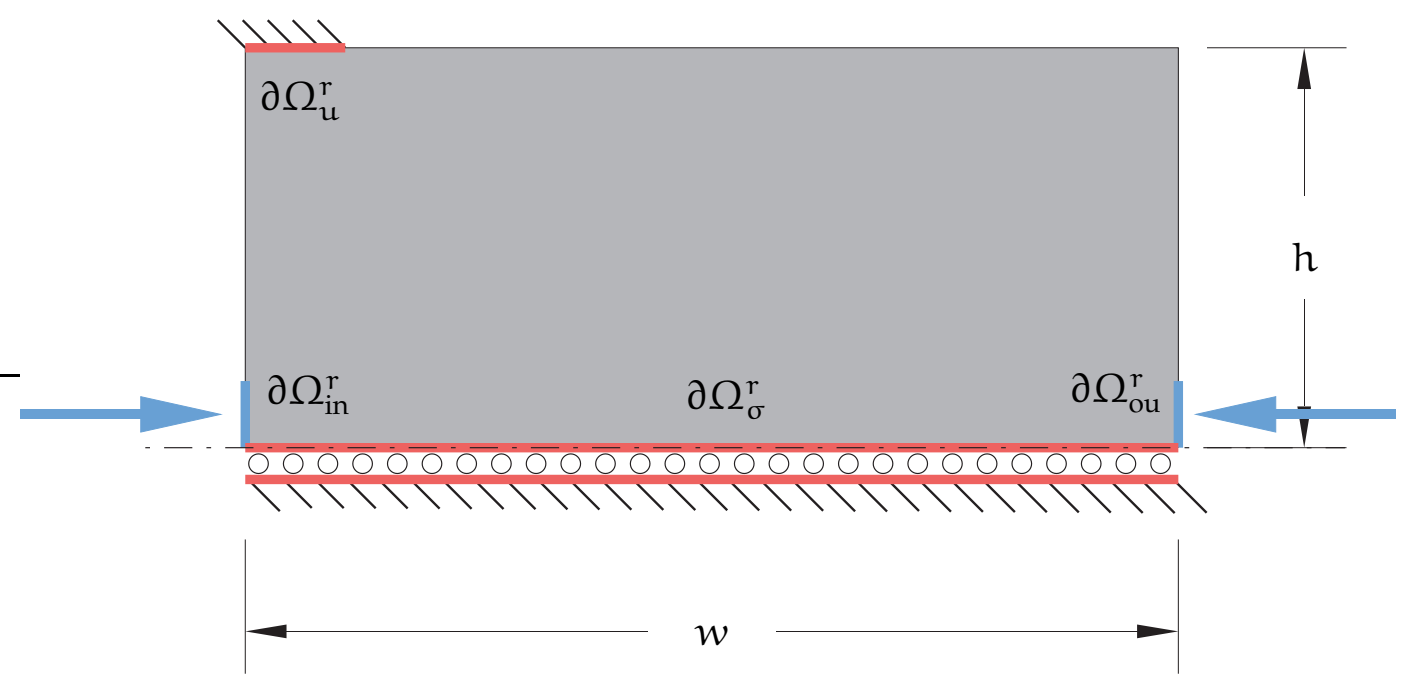

Figura 5.26: Configuração do domínio fixo estendido para à síntese de um mecanismo flexível inversor.

Na região $\partial \Omega_{\text {in }}^{r}$ é aplicado um carregamento superficial de $100,0 \mathrm{~N} / \mathrm{mm}^{2}$ e em $\partial \Omega_{\text {ou }}^{\mathrm{r}}$ o carregamento é de $-20,0 \mathrm{~N} / \mathrm{mm}^{2}$. As regiões $\partial \Omega_{0}^{r}$ e $\partial \Omega_{1}^{r}$ são relativas as condições de contorno de Dirichlet, onde $\partial \Omega_{0}^{r}$ é referente a fixação $(\mathbf{u}=\mathbf{0})$ e simetria no eixo y 
$\left(u_{1}=0\right)$ respetivamente.

O valor penalização utilizada no modelo de material SIMP foi de 3,0 , sendo este o valor que foram obtidos os melhores resultados.

Definido, portanto todas as condições e parâmetros relativos a solução do problema de equilíbrio via MEF, podemos agora definir o problema relativo a distribuição de material pelo MOT com a finalidade de projetar esse mecanismo biestável:

$$
\begin{array}{cl}
\underset{\rho_{e} \in[0.0,1.0]}{\operatorname{Min} .} & \Theta^{(l c=1)}+\Theta^{(l c=2)}=\sum_{l c}^{2} \sum_{t}^{n_{t}(t)} \mathbf{f}_{e x t} \cdot{ }^{(t)} \mathbf{u} \\
\text { Sujeito à } & \delta \Pi_{p}\left(\rho_{e}, u, \delta \boldsymbol{u}\right)=0,0 \\
& \left({ }_{\left.t_{i}\right)} \bar{\lambda}^{l c=1}=\{0,6 ; 0,4 ; 0,8\}\right. \\
& \bar{V}^{r}\left(\rho_{e}\right)-\underline{V} \leqslant 0 \\
& \int_{\partial \Omega_{\text {ou }}^{r}} \mathbf{h}_{0} \cdot \mathbf{u} d a^{r} \leqslant-50,0 \\
& 12,5 \mathrm{~mm}=r_{\min } \leqslant r^{*}\left(\Omega_{d}^{r}\right)
\end{array}
$$

onde restrição de deslocamento da porta de saída do mecanismo é definida como uma integral de superfície em $\partial \Omega_{\text {ou }}^{r}$ dos deslocamentos $\mathbf{u}$ no caso de carregamento lc $=1$ e pseudo-tempo $t=1,0$ com o produto interno de do vetor de seleção $\mathbf{h}_{0}$ dos graus de liberdade em $x_{0}$, ou seja, é equivalente a uma restrição de deslocamento no $-10,0 \mathrm{~mm}$ em cada nó contido em $\partial \Omega_{\mathrm{ou}}^{\mathrm{r}}$. A restrição do incremento de carga é imposta no primeiro caso de carregamento $\left({ }^{(t)} \bar{\lambda}^{l c=1}\right)$, onde os valores de pseudo-tempo selecionados são de $\{0,5 ; 0,7 ; 0,9\}$.

A função objetivo selecionada é refente a energia de deformação externa e os incrementos de pseudo-tempo selecionados para o primeiro caso de carregamento foram $\{0,5 ; 0,7 ; 0,9 ; 1,0\}$. Para o segundo caso de carregamento foi de $\{0,5 ; 0,7 ; 0,9 ; 1,0\}$.

A restrição de volume de volume selecionado foi 40,00\% em relação ao volume total. A topologia final da estrutura do mecanismo flexível inversor obtido pode ver verificada na figura 5.2 . 

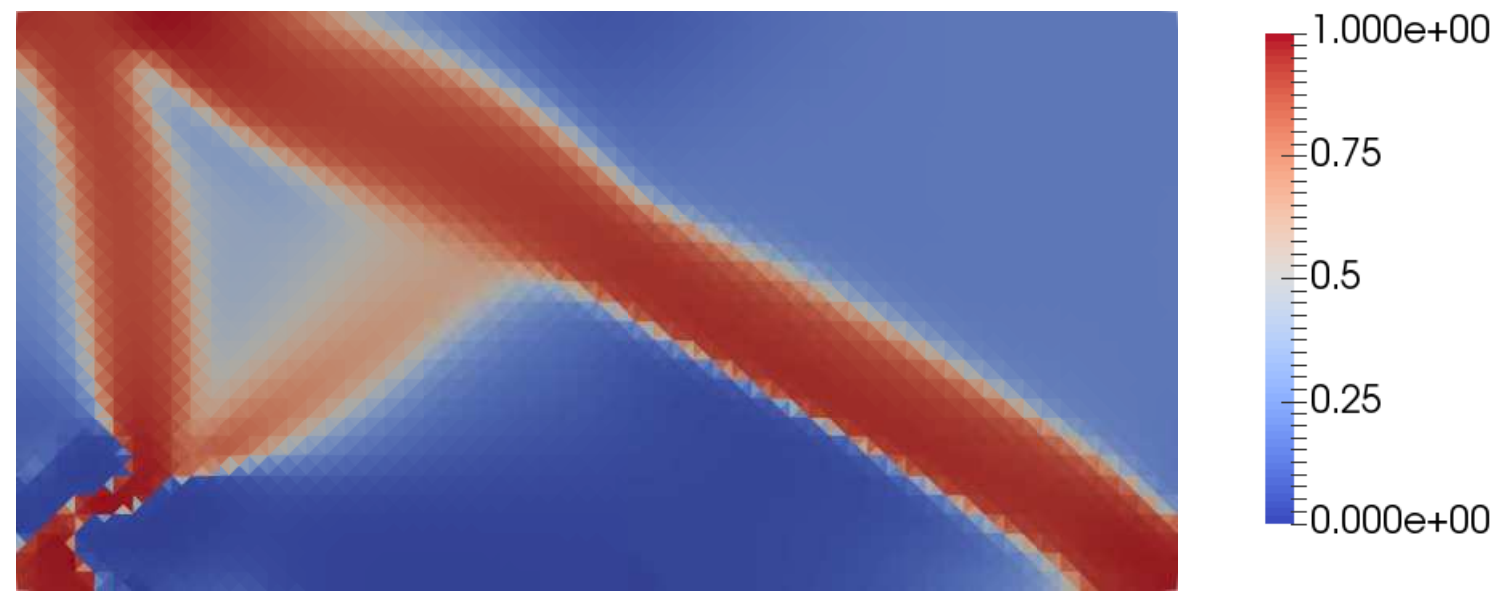

Figura 5.27: Topologia do mecanismo flexível biestável inversor considerando a restrição de volume em $40 \%$.

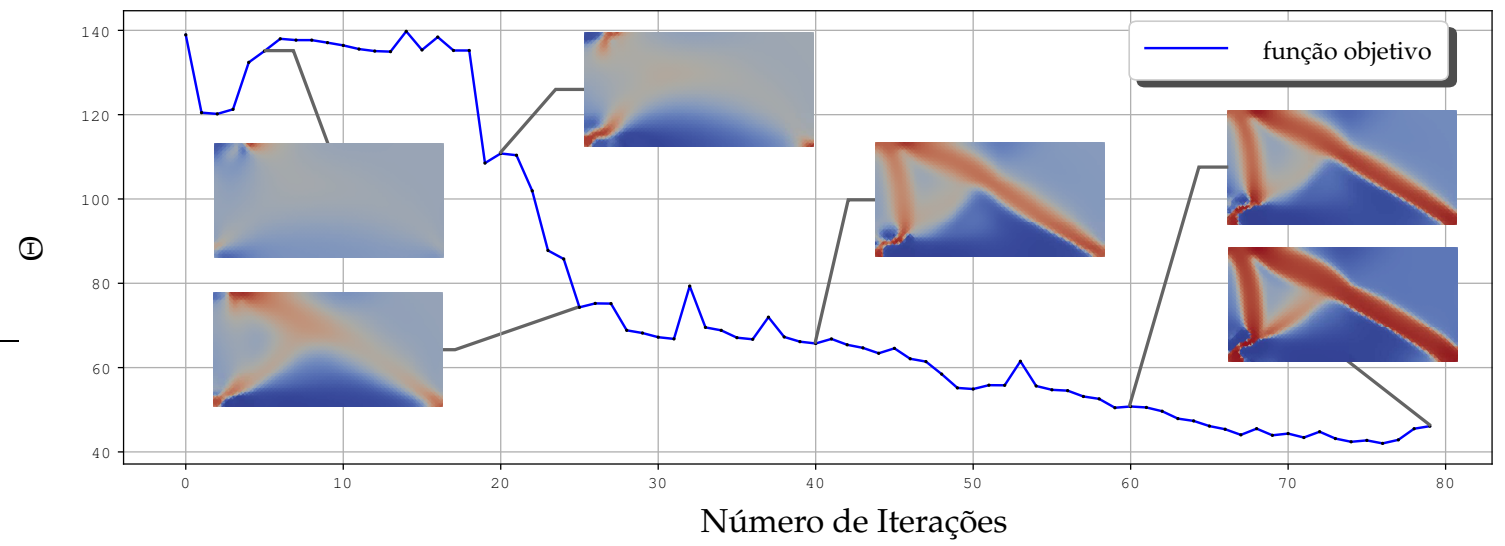

Figura 5.28: Evolução da função objetivo considerando a restrição de volume em $60 \%$.

Nas figuras 5.28, 5.29 e 5.30 são apresentados gráficos referentes a função objetivo e de restrições referentes a convergência e evolução do problema. A evolução da topologia da estrutura são apontadas nas iterações 5, 15, 25, 50, 75 e 150 da figura 5.28.

Os gráficos referentes ao caminho de carga da estrutura em relação aos deslocamentos e pseudo-tempo na região $\partial \Omega_{\text {in }}^{r}$ são apresentados na figura 5.31 , onde é possível verificar a violação das restrições de incremento de carga respectivamente.

Foi feita uma interpretação da topologia final somente removendo elementos dentro dum valor de pseudo densidade que minimizasse o erro entre o modelo original. O modelo interpretado utilizando como função de erro a somatória das funções de restrições. O resultado da topologia é apresentado na figura 5.32 . 


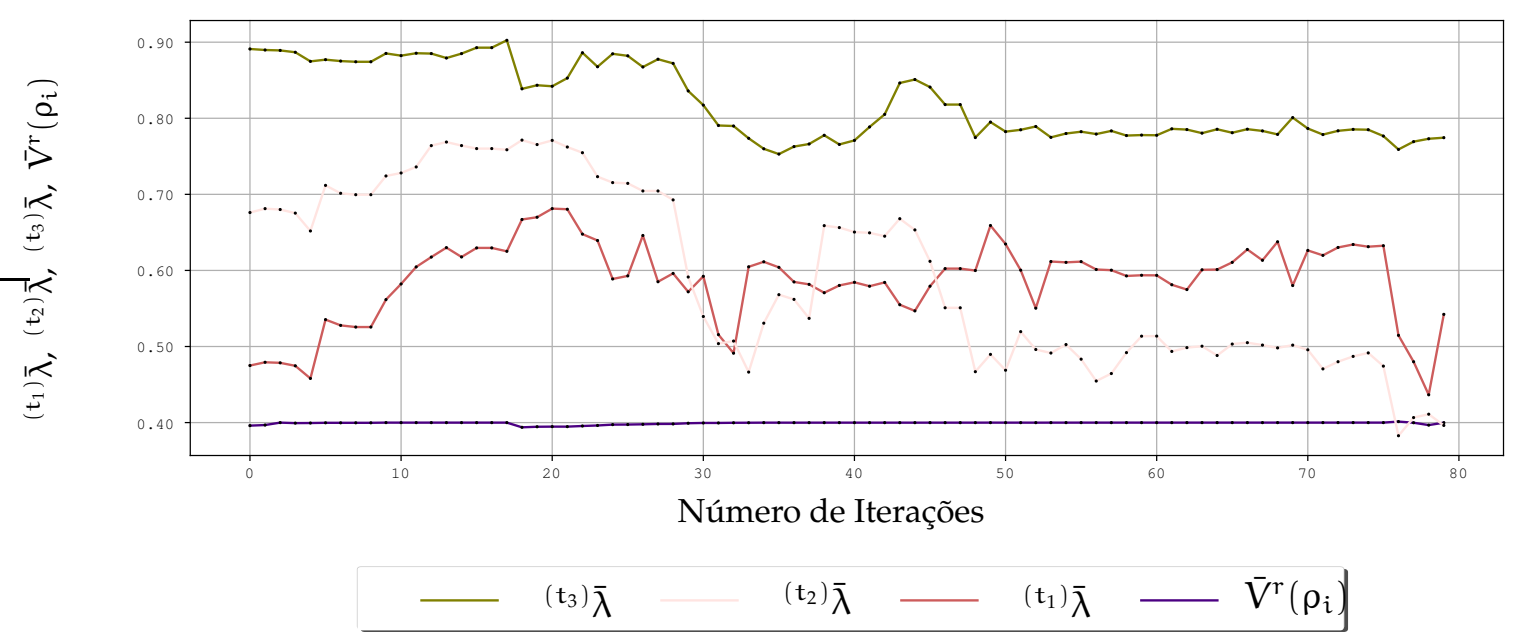

Figura 5.29: Evolução das funções de restrições de incremento de carga e fração de volume.

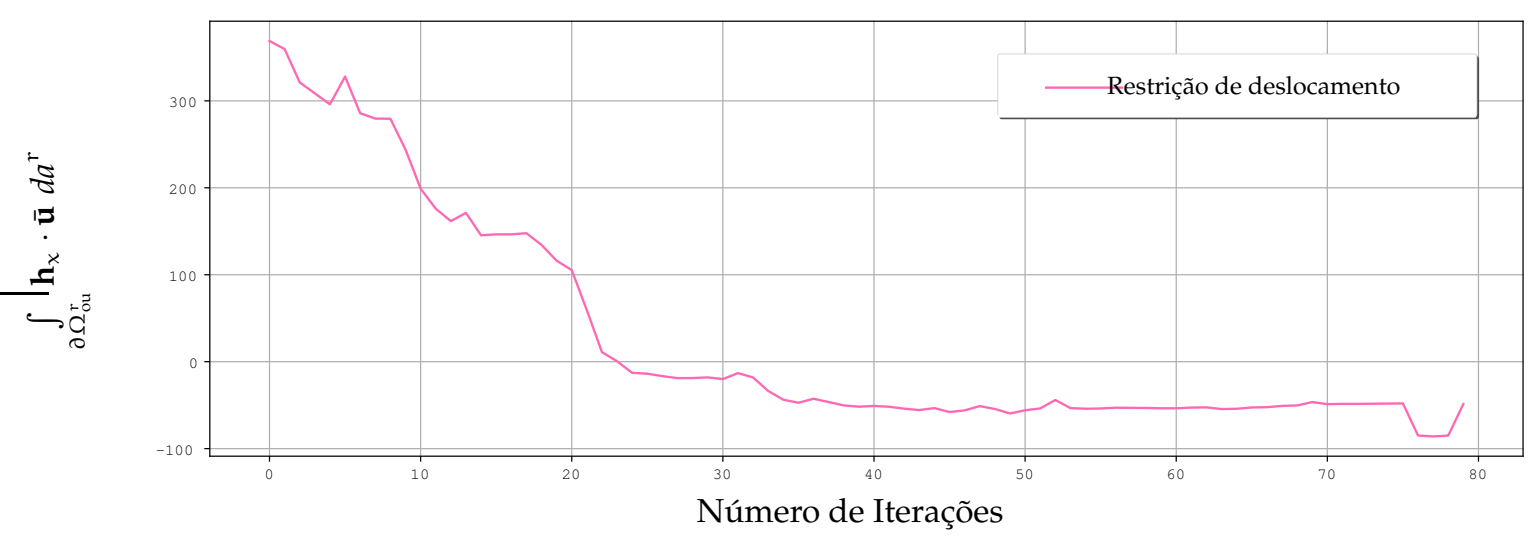

Figura 5.30: Evolução da restrição de deslocamento em $\partial \Omega_{\mathrm{ou}}^{\mathrm{r}}$.

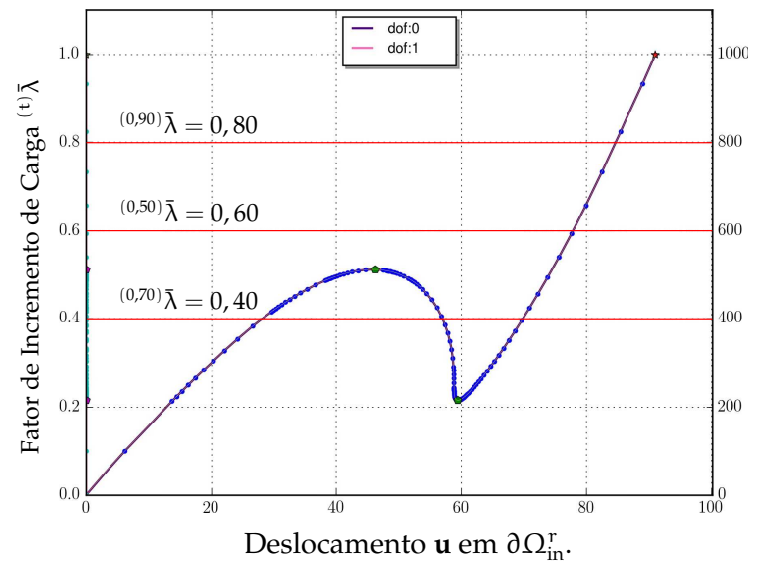

(a)

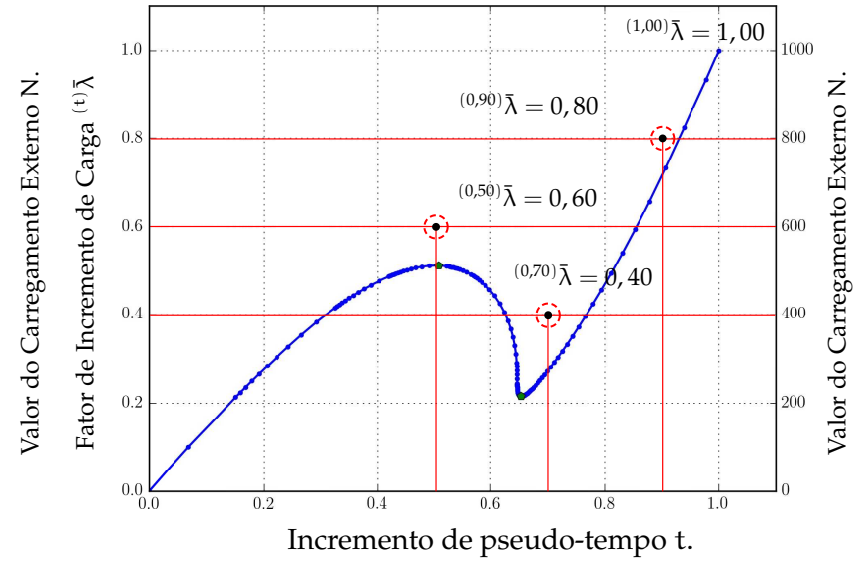

(b)

Figura 5.31: Gráfico de incremento de carga em relação ao deslocamento (a) e pseudo-tempo (b) considerando a restrição de volume em $40 \%$. 


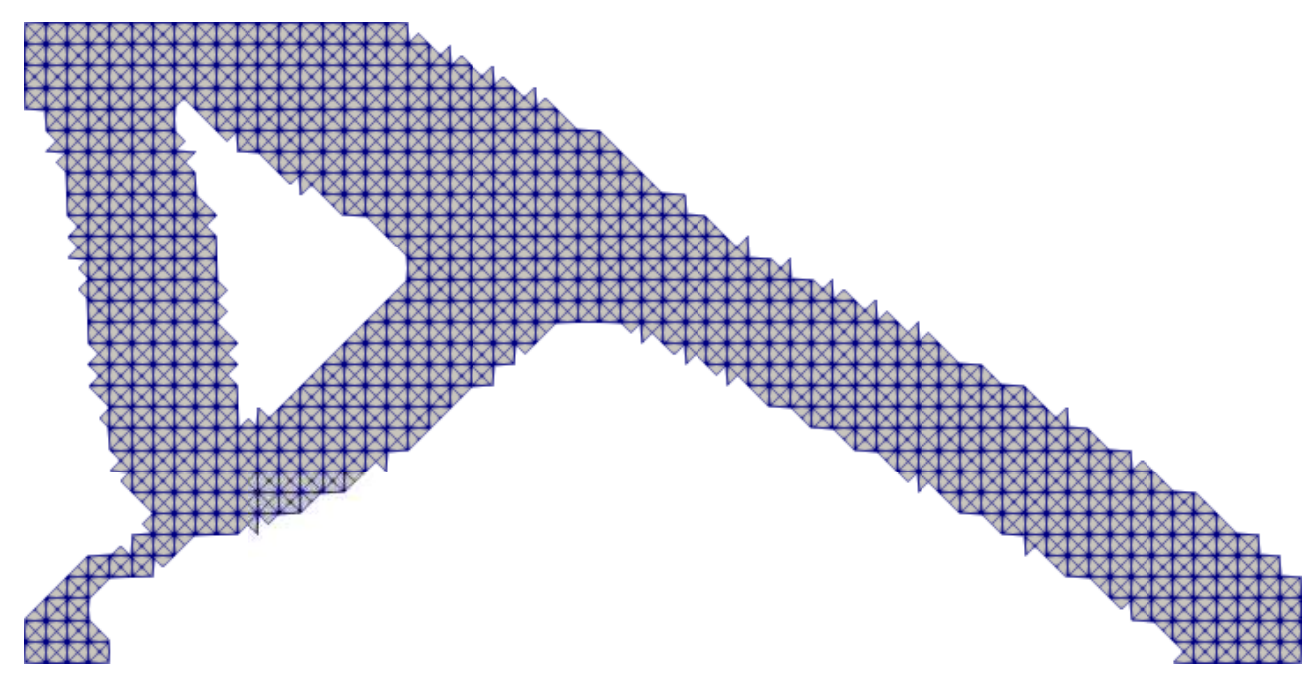

Figura 5.32: Malha da interpretação dos resultados para um mecanismo inversor considerando a restrição de volume em $40 \%$.

Uma verificação do desempenho do mecanismo biestável inversor em relação à interpretação obtida foi feita e os gráficos com os resultados referentes ao caminho de carga da estrutura em relação aos deslocamentos e pseudo tempo na região $\Omega_{\text {in }}^{r}$ são apresentados na figura 5.19, onde é possível verificar que as restrições do fator de incremento de carga não se mantiveram próximas em comparação as apresentadas na figura 5.17 .

No entanto falta a verificação dos deslocamentos na porta de saída do mecanismo, esta verificação é apresentada na figura 5.34, onde é possível verificar que o valor dos deslocamentos estão próximos aos valores das restrições (5.4), ou seja próximos de $-10,0 \mathrm{~mm}$ no pseudo-tempo $\mathrm{t}=1,0$. 


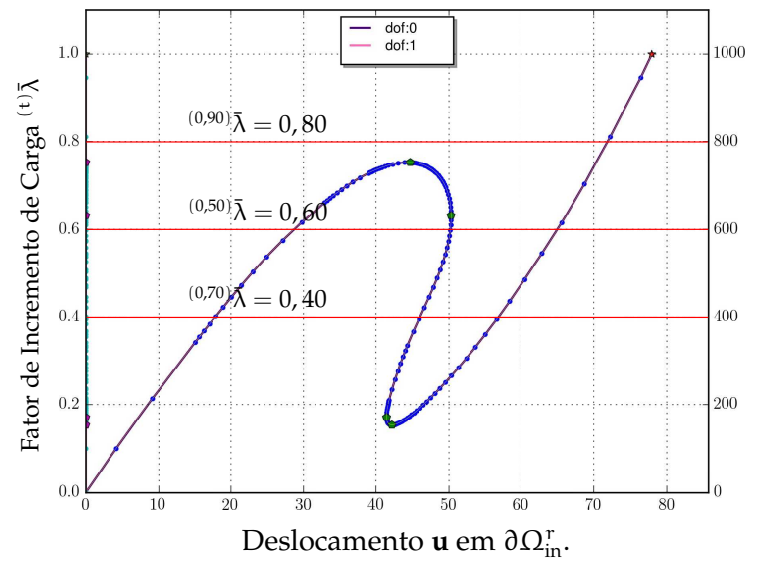

(a)
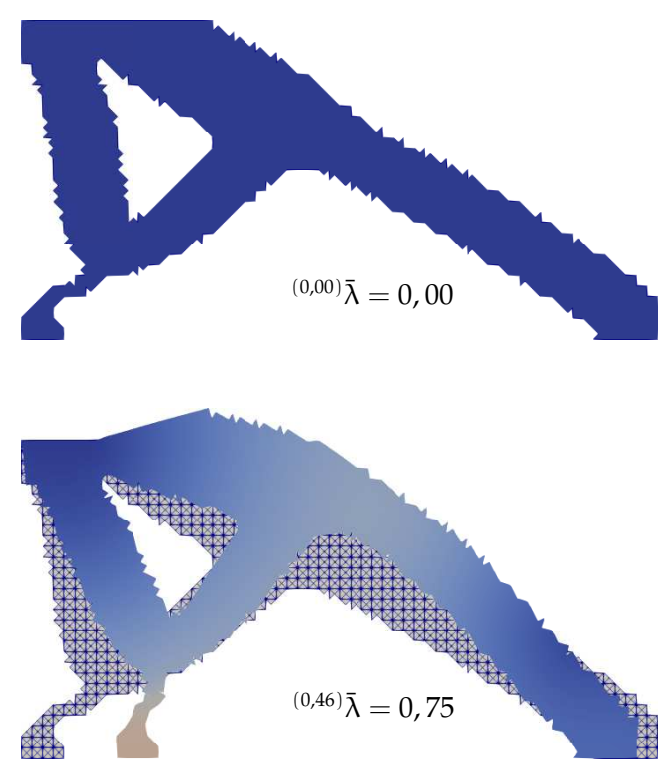

(c)

Figura 5.33: Gráfico de incremento de carga em relação ao deslocamento (a) e pseudo-tempo (b), configuração deformada (c) do modelo interpretado considerando a restrição de volume em $40 \%$.
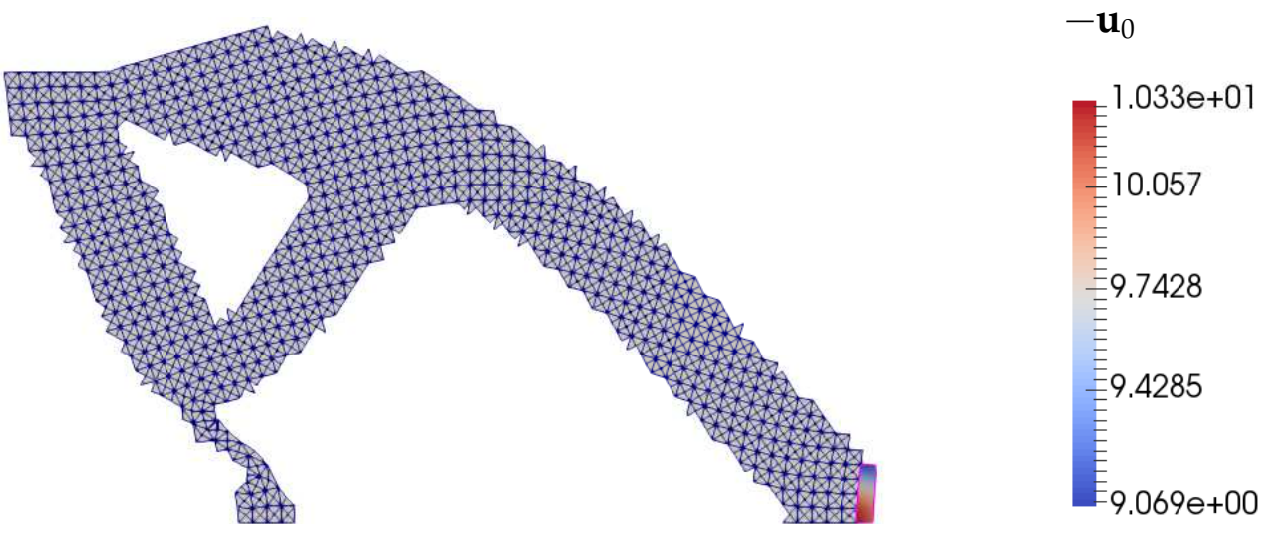

Figura 5.34: Avaliação dos deslocamentos relativo ao resultado obtido para um mecanismo biestável inversor considerando a restrição de volume em $40 \%$. 


\section{Capítulo 6}

\section{Conclusões}

A metodologia desenvolvida nesta tese obteve um caminho claro para o desenvolvimento de mecanismos flexíveis biestáveis utilizando o MOT, onde foi necessário utilizar uma equação elástica constitutiva policonvexa, com um algoritmo de otimização numérico baseado no Método de Ponto Interior em conjunto com o Método dos Mínimos Quadrados, para alargar o domínio viável inicial utilizando derivadas do fator de incremento de carga com base na configuração inicial do comprimento de arco da solução não linear. No entanto, ainda é necessária melhorias na metodologia para projetar mecanismos flexíveis onde a porta de entrada é diferente da porta de saída, mais especificamente no caso de inversores, onde os resultados se apresentaram instáveis.

A formulação desenvolvida neste trabalho é descrita pelas equações (4.31), (5.2) e (5.4) impõem restrições de deslocamento e de incremento de carga via Método dos Mínimos Quadrados (MMQ) em conjunto ao Método de Ponto Interior com o objetivo de estabilizar e estimar as variáveis duais fora do domínio viável, ou seja, criando domínio viável alargado e trazendo de forma gradativa as restrições para dentro dos limites de projeto. Esta metodologia se demonstrou um ingrediente fundamental que possibilitou estabilizar e obter uma solução factível, com pouca escala de cinza para mecanismos biestáveis sem restrição de deslocamento, conforme apresentado na seção 5.1. No entanto, só foi apresentado um exemplo de mecanismos flexíveis biestáveis inversores, devido à instabilidade de solução, credita-se esta instabilidade a inclusão da 
restrição de deslocamento.

Comparando os resultados de mecanismos flexíveis biestáveis obtidos neste trabalho em relacão aos da literatura (Bruns et al., 2002a; Bruns e Sigmund, 2004; James e Waisman, 2016) é possível constatar a clareza dos resultados obtidos, como baixa escala de cinza e boa definição das dobradiças ou "hinges". Portanto, pela primeira vez foi possível impor restrições de projeto relativa ao fator de incremento de carga em pontos pré-determinados na trajetória de equilíbrio da estrutura utilizando MOTpara projetar estruturas que utilizassem o mecanismo de flambagem não linear (Bruns et al., 2002a; Bruns e Sigmund, 2004; Ohsaki e Nishiwaki, 2005; Lindgaard e Dahl, 2013; Prasad e Diaz, 2006; James e Waisman, 2016). Para uma melhor clareza e entendimento dos resultados obtidos, na figura 6.1 é apresentada uma comparação das topologias supra mencionadas.

Avaliou-se a maioria dos métodos de otimização numéricos mais utilizados na literatura para aplicação no MOT, como CO, एPLS, CONLIN, MAM, IpOpt, Foi analisado inicialmente o desempenho em lidar com restrições não lineares, como deslocamento e foi verificado que o melhor desempenho em relação aos demais métodos era da implementação IpOpt. Chegou-se a esta conclusão na metade do ano de 2015, quando já havia sido trocada a plataforma de trabalho MATLAB para a plataforma FEniCS(Logg et al., 2012). No mesmo período outro trabalho foi desenvolvido por Rojas-Labanda e Stolpe (2015), cujo tema era direcionado a testes e comparações de diversos algoritmos de otimização numérico aplicado ao MOT também e conclusão deste trabalho é enfática: o IpOpt obteve um desempenho muito superior ao MAM em todos os testes, onde foram comparados valores da função objetivo e estabilidade da solução durante o processo iterativo de OT. Estas conclusões de Rojas-Labanda e Stolpe (2015) corroboram com as decisões tomadas durante o desenvolvimento desta tese. No entanto, este trabalho vai além e apresenta fortes indícios numéricos que o IpOpt também é superior em trabalhar com restrições não-lineares envolvendo problemas aplicados a OT, no caso restrições de deslocamento e de fator de incremento de carga proporcional, tema ainda não abordado na literatura até a presente data (junho de 2017). 


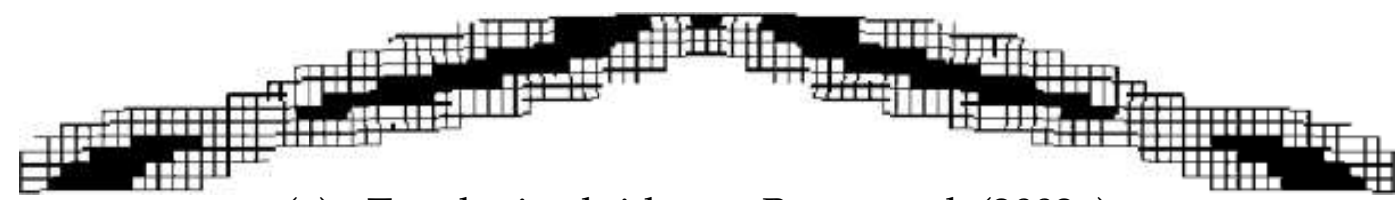

(a) - Topologia obtida por Bruns et al. (2002a).

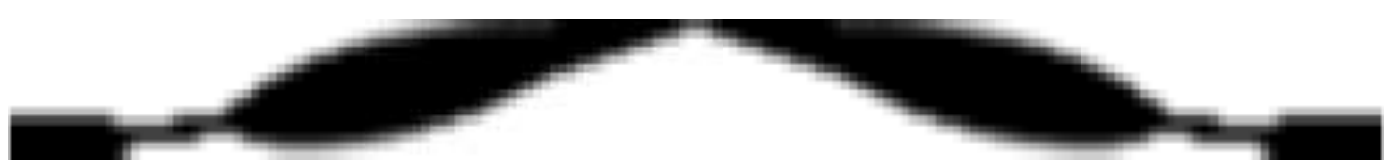

(b) - Topologia obtida por Iames e Waisman (2016).

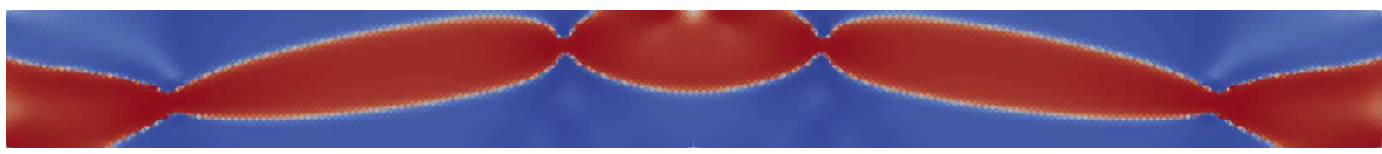

(c) - Topologia obtida utilizando a metodologia proposta, iteração 75.

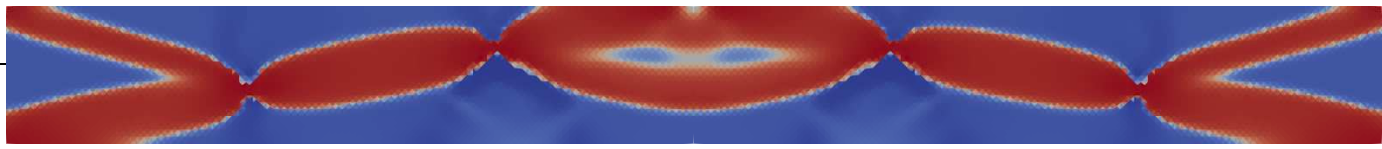

(d) - Topologia final obtida utilizando a metodologia proposta, iteração 150.

Figura 6.1: Comparação entres os resultados da literatura com os resultados obtidos com a metodologia desenvolvida neste trabalho.

\subsection{Trabalhos Futuros}

A continuação natural do trabalho desenvolvido neste tese é estendê-los com o intuito de melhor o elemento finito utilizado, o método de otimização e com a popularização das impressoras 3Ds, projetar estruturas instáveis compostas por diversos materiais.

Lista de itens de interesse em estudos futuros:

- Decomposição recursiva para otimização não-convexa: Outro campo importante de estudo na área relativa a flambagem não linear está relacionada ao estudo do algoritmo de otimização numérico, este tipo de problema é não convexo e para melhorar a convergência deseja-se estudar o Método da Decomposição Recursiva.

- Método dos Elementos Finitos Mistos: Mesmo com a utilização de equações 
constitutivas policonvexas, ainda existe a necessidade de melhor a estabilidade do elemento finito quando sujeito a grandes deformações e um meio de melhorar é através da adição variáveis primais extras ao problema, como variáveis referente ao campo de pressão interna, campo do gradiente da transformação e o tensor do gradiente da transformação, no caso aplicar o funcional misto de $\mathrm{Hu}$-Washizu ou similar.

- Projeto de Mecanismos Flexíveis Multi-Material: O projeto de mecanismos flexíveis envolve o problema de dobradiças ou hinges que em alguns casos se apresentam como uma única ligação nodal entre os membros da estrutura. A flexibilização do número de materiais durante o processo de OT, pode permitir uma modelagem mais precisa das regiões de dobradiças com materiais de propriedades similares a elastômeros, possibilitando a obtenção de mecanismos com respostas mais complexas. 


\section{Referências Bibliográficas}

Alnæs M., Blechta J., Hake J., Johansson A., Kehlet B., Logg A., Richardson C., Ring J., Rognes M. E., Wells G. N., The fenics project version 1.5, Archive of Numerical Software, 2015, vol. 3, p. 9

Altenbach H., Yuriy I. Dimitrienko, Nonlinear Continuum Mechanics and Large Inelastic Deformations, Springer: Dordrecht, Heidelberg, London, New York, 2012. Wiley Online Library, 2012

Ananthasuresh G. K., A new design paradigm for micro-electro-mechanical systems and investigations on the compliant mechanisms synthesis., 1994, Tese de Doutorado

Anton S. R., Sodano H. A., A review of power harvesting using piezoelectric materials (2003-2006), Smart materials and Structures, 2007, vol. 16, p. R1

Ball J. M., Convexity conditions and existence theorems in nonlinear elasticity, Archive for Rational Mechanics and Analysis, 1976, vol. 63, p. 337

Bažant Z. P., Cedolin L., Stability of structures: elastic, inelastic, fracture and damage theories. World Scientific, 2010

Bendsøe M. P., Kikuchi N., Generating optimal topologies in structural design using a homogenization method, Computer Methods in Applied Mechanics and Engineering, 1988, vol. 71, p. 197

Bendsøe M. P., Optimal Shape Design as a Material Distribution Problem, Structural Optimization, 1989, vol. 1, p. 192 
Bendsøe M. P., Optimization of structural topology, shape, and material. vol. 2, Springer, 1995

Bendsøe M. P., Sigmund O., Material interpolation schemes in topology optimization, Archive of applied mechanics, 1999, vol. 69, p. 635

Bendsoe M. P., Sigmund O., Topology optimization: theory, methods, and applications. Springer Science \& Business Media, 2013

Bertram A., Elasticity and plasticity of large deformations. Springer, 2005

Bonet J., Wood R. D., Nonlinear continuum mechanics for finite element analysis. Cambridge university press, 1997

Bourdin B., Filters in topology optimization, International Journal for Numerical Methods in Engineering, 2001, vol. 50, p. 2143

Bruns T., Sigmund O., Toward the topology design of mechanisms that exhibit snapthrough behavior, Computer Methods in Applied Mechanics and Engineering, 2004, vol. 193 , p. 3973

Bruns T., Sigmund O., Tortorelli D. A., Numerical methods for the topology optimization of structures that exhibit snap-through, International Journal for Numerical Methods in Engineering, 2002a, vol. 55, p. 1215

Bruns T. E., Sigmund O., Tortorelli D. A., Numerical methods for the topology optimization of structures that exhibit snap-through, International Journal for Numerical Methods in Engineering, 2002b, vol. 55, p. 1215

Bruns T. E., Tortorelli D. A., Topology optimization of non-linear elastic structures and compliant mechanisms, Computer Methods in Applied Mechanics and Engineering, 2001, vol. 190, p. 3443

Buhl T., Pedersen C. B., Sigmund O., Stiffness design of geometrically nonlinear structures using topology optimization, Structural and Multidisciplinary Optimization, 2000, vol. 19 , p. 93

Byrd R. H., Hribar M. E., Nocedal J., An interior point algorithm for large-scale nonlinear programming, SIAM Journal on Optimization, 1999, vol. 9, p. 877 
Campello E., Pimenta P., Wriggers P., A triangular finite shell element based on a fully nonlinear shell formulation, Computational Mechanics, 2003, vol. 31, p. 505

Capozzoli M., Gopalakrishnan J., Hogan K., Massad J. E., Tokarchik T., Wilmarth S., Banks H. T., Mossi K. M., Smith R. C., Modeling aspects concerning THUNDER actuators. In 1999 Symposium on Smart Structures and Materials , 1999, p. 719

Carbonari R. C., Silva E. C., Paulino G. H., Topology optimization design of functionally graded bimorph-type piezoelectric actuators, Smart Materials and Structures, 2007, vol. 16, p. 2605

Cardoso E., Fonseca J., The GDC method as an orthogonal arc-length method, Communications in numerical methods in engineering, 2007, vol. 23, p. 263

Cardoso E. L., Fonseca J. S. O., Strain energy maximization approach to the design of fully compliant mechanisms using topology optimization, Latin American Journal of Solids and Structures, an ABCM Journal, 2004, vol. 1, p. 263

Cardoso E. L., Fonseca J. S. O., Topology Optimization of Piezoelectric Actuators Considering Geometric Nonlinearities. In IUTAM Symposium on Topological Design Optimization of Structures, Machines and Materials , 2006, p. 391

Carr J. C., Beatson R. K., Cherrie J. B., Mitchell T. J., Fright W. R., McCallum B. C., Evans T. R., Reconstruction and representation of $3 \mathrm{D}$ objects with radial basis functions. In Proceedings of the 28th annual conference on Computer graphics and interactive techniques , 2001, p. 67

Ciarlet P. G., Mathematical Elasticity 1 edn. vol. 1, Elsevier Science North Holland, Amsterdam, 1988a

Ciarlet P. G., Preface. vol. 20, Elsevier, 1988b, ix

Coleman B. D., Noll W., On the thermostatics of continuous media, Archive for rational mechanics and analysis, 1959 , vol. 4, p. 97

Crawley E. F., De Luis J., Use of piezoelectric actuators as elements of intelligent structures, AIAA journal, 1987, vol. 25, p. 1373 
Dantzig G. B., Thapa M. N., Linear programming 2: theory and extensions. Springer Science \& Business Media, 2006

Fleury C., CONLIN: an efficient dual optimizer based on convex approximation concepts, Structural optimization, 1989, vol. 1, p. 81

Franke R., Scattered data interpolation: Tests of some methods, Mathematics of computation, 1982, vol. 38, p. 181

Gea H. C., Luo J., Topology optimization of structures with geometrical nonlinearities, Computers \& Structures, 2001, vol. 79, p. 1977

Griewank A., Juedes D., Utke J., Algorithm 755: ADOL-C: a package for the automatic differentiation of algorithms written in $\mathrm{C} / \mathrm{C}++, \mathrm{ACM}$ Transactions on Mathematical Software (TOMS), 1996, vol. 22, p. 131

Groenwold A. A., Etman L., On the equivalence of optimality criterion and sequential approximate optimization methods in the classical topology layout problem, International journal for numerical methods in engineering, 2008, vol. 73, p. 297

Haber R., Jog C., Bendsøe M. P., A new approach to variable-topology shape design using a constraint on perimeter, Structural Optimization, 1996, vol. 11, p. 1

Haftka R. T., Gürdal Z., Elements of structural optimization. vol. 11, Springer Science \& Business Media, 2012

Holzapfel A. G., Nonlinear Solid Mechanics II. John Wiley \& Sons, Inc., 2000

Howard M., Pajot J., Maute K., Dunn M. L., A computational design methodology for assembly and actuation of thin-film structures via patterning of eigenstrains, Journal of Microelectromechanical Systems, 2009, vol. 18, p. 1137

$\mathrm{Hu}$ N., et al., Buckling-induced smart applications: recent advances and trends, Smart Materials and Structures, 2015, vol. 24, p. 063001

Hughes T. J., The finite element method: linear static and dynamic finite element analysis. Courier Corporation, 2012

Ikeda T., Fundamentals of piezoelectricity. Oxford university press, 1996 
James K. A., Waisman H., Layout design of a bi-stable cardiovascular stent using topology optimization, Computer Methods in Applied Mechanics and Engineering, 2016, vol. 305, p. 869

Jog C., Distributed-parameter optimization and topology design for non-linear thermoelasticity, Computer Methods in Applied Mechanics and Engineering, 1996, vol. 132, p. 117

Jonsmann J., Technology Development for Topology Optimised Thermal Microactuators. MIC, 2000

Jung S.-M., Yun K.-S., Energy-harvesting device with mechanical frequency-up conversion mechanism for increased power efficiency and wideband operation, Applied Physics Letters, 2010, vol. 96, p. 111906

Kasper E., Taylor R., Mixed-enhanced formulation for finite deformation axisymmetrictorsion, International journal for numerical methods in engineering, 2004, vol. 60, p. 341

Kemmler R., Lipka A., Ramm E., Large deformations and stability in topology optimization, Structural and Multidisciplinary Optimization, 2005, vol. 30, p. 459

Kikuchi N., Kota S., Topological synthesis of compliant mechanisms using multi-criteria optimization, 1997

Kögl M., Bucalem M., Analysis of smart laminates using piezoelectric MITC plate and shell elements, Computers \& structures, 2005, vol. 83, p. 1153

Kögl M., Silva E. C., Topology optimization of smart structures: design of piezoelectric plate and shell actuators, Smart materials and Structures, 2005, vol. 14, p. 387

Kuhl E., Askes H., Steinmann P., An illustration of the equivalence of the loss of ellipticity conditions in spatial and material settings of hyperelasticity, European Journal of Mechanics-A/Solids, 2006, vol. 25, p. 199

Lahuerta R. D., Simões E. T., Campello E. M. B., Pimenta P. M., Silva E. C. N., Towards the stabilization of the low density elements in topology optimization with large deformation, Computational Mechanics, 2013, vol. 52, p. 779 
Larsen U. D., Signund O., Bouwsta S., Design and fabrication of compliant micromechanisms and structures with negative Poisson's ratio, Journal of Microelectromechanical Systems, 1997, vol. 6, p. 99

Lee S.-L., Manuel F., Large deflections and stability of elastic frames, 1968

Leipholz H., Stability theory: An introduction to the stability of dynamic systems and rigid bodies. Springer-Verlag, 2013

Lindgaard E., Dahl J., On compliance and buckling objective functions in topology optimization of snap-through problems, Structural and Multidisciplinary Optimization, 2013, vol. 47, p. 409

Logg A., Mardal K.-A., Wells G., Automated solution of differential equations by the finite element method: The FEniCS book. vol. 84, Springer Science \& Business Media, 2012

Luo Z., Zhang N., Wang Y., Gao W., Topology optimization of structures using meshless density variable approximants, International Journal for Numerical Methods in Engineering, 2013, vol. 93, p. 443

Lyapunov A. M., The general problem of the stability of motion, International Journal of Control, 1992, vol. 55, p. 531

Marsden J. E., Hughes T. J., Mathematical foundations of elasticity. Courier Corporation, 1994

Meressi T., Paden B., Buckling control of a flexible beam using piezoelectric actuators, Journal of Guidance, Control, and Dynamics, 1993, vol. 16, p. 977

Morrey C. B., et al., Quasi-convexity and the lower semicontinuity of multiple integrals, Pacific J. Math, 1952, vol. 2, p. 25

Mossi K. M., Bishop R. P., Characterization of different types of high-performance THUNDER actuators. In 1999 Symposium on Smart Structures and Materials , 1999, p. 43 
Nishiwaki S., Frecker M. I., Min S., Kikuchi N., Topology optimization of compliant mechanisms using the homogenization method, International Journal for Numerical Methods in Engineering, 1998, vol. 42, p. 535

Nocedal J., Wright S., Numerical optimization. Springer Science \& Business Media, 2006

Noguchi H., Hisada T., Sensitivity analysis in post-buckling problems of shell structures, Computers \& structures, 1993, vol. 47, p. 699

Ohsaki M., Nishiwaki S., Shape design of pin-jointed multistable compliant mechanisms using snapthrough behavior, Structural and Multidisciplinary Optimization, 2005, vol. 30 , p. 327

Pedersen C. B., Buhl T., Sigmund O., Topology synthesis of large-displacement compliant mechanisms, International Journal for numerical methods in engineering, 2001, vol. 50, p. 2683

Penzler P., Rumpf M., Wirth B., A phase-field model for compliance shape optimization in nonlinear elasticity, ESAIM: Control, Optimisation and Calculus of Variations, 2012, vol. 18 , p. 229

Pimenta P. M., Fundamentos da Mecânica dos Sólidos e das Estruturas 1 edn. USP São Paulo, 2008

Portela P., Camanho P., Weaver P., Bond I., Analysis of morphing, multi stable structures actuated by piezoelectric patches, Computers \& Structures, 2008, vol. 86, p. 347

Prasad J., Diaz A., Synthesis of bistable periodic structures using topology optimization and a genetic algorithm, Journal of mechanical design, 2006, vol. 128, p. 1298

Riks E., An incremental approach to the solution of snapping and buckling problems, International Journal of Solids and Structures, 1979, vol. 15, p. 529

Rippa S., An algorithm for selecting a good value for the parameter c in radial basis function interpolation, Advances in Computational Mathematics, 1999, vol. 11, p. 193

Rojas-Labanda S., Stolpe M., Benchmarking optimization solvers for structural topology optimization, Structural and Multidisciplinary Optimization, 2015, vol. 52, p. 527 
Schwartz R. W., Narayanan M., Development of high performance stress-biased actuators through the incorporation of mechanical pre-loads, Sensors and Actuators A: Physical, 2002, vol. 101, p. 322

Schweizerhof K. H., Wriggers P., Consistent linearization for path following methods in nonlinear FE analysis, Computer Methods in Applied Mechanics and Engineering, 1986 , vol. 59 , p. 261

Seo Y.-D., Kim H.-J., Youn S.-K., Shape optimization and its extension to topological design based on isogeometric analysis, International Journal of Solids and Structures, 2010, vol. 47, p. 1618

Sigmund O., On the design of compliant mechanisms using topology optimization, Journal of Structural Mechanics, 1997, vol. 25, p. 493

Silva E. C. N., Topology optimization applied to the design of linear piezoelectric motors, Journal of intelligent material systems and structures, 2003, vol. 14, p. 309

Silva E. C. N., Kikuchi N., Design of piezoelectric transducers using topology optimization, Smart Materials and Structures, 1999, vol. 8, p. 350

Silva E. N., Nishiwaki S., Kikuchi N., Topology optimization design of flextensional actuators, IEEE transactions on ultrasonics, ferroelectrics, and frequency control, 2000, vol. 47 , p. 657

Simo J. C., Hughes T. R. J., Plasticity and viscoplasticity: Numerical analysis and computational aspects. Springer-Verlag Berlin, 1991

Starnes Jr J. H., Haftka R. T., Preliminary design of composite wings for buckling, strength, and displacement constraints, Journal of Aircraft, 1979, vol. 16, p. 564

Suzuki K., Kikuchi N., A homogenization method for shape and topology optimization, Computer methods in applied mechanics and engineering, 1991, vol. 93, p. 291

Svanberg K., The method of moving asymptotes-a new method for structural optimization, International journal for numerical methods in engineering, 1987, vol. 24, p. 359 
Tawfik S. A., Dancila D. S., Armanios E., Unsymmetric composite laminates morphing via piezoelectric actuators, Composites Part A: Applied Science and Manufacturing, 2011, vol. 42, p. 748

Treloar L., The elasticity of a network of long-chain molecules-II, Transactions of the Faraday Society, 1943, vol. 39, p. 241

Uchino K., Advanced piezoelectric materials: Science and technology. Elsevier, 2010

Vanderplaats G. N., Numerical optimization techniques for engineering design: with applications. vol. 1, McGraw-Hill New York, 1984

Wallin M., Ristinmaa M., Finite strain topology optimization based on phase-field regularization, Structural and Multidisciplinary Optimization, 2015, vol. 51, p. 305

Wriggers P., Nonlinear Finite Element Methods 1 edn. Springer-Verlag Berlin Heidelberg Berlin, 2008

Wu C., Arora J., Design sensitivity analysis of non-linear buckling load, Computational Mechanics, 1988, vol. 3, p. 129

Yang Y.-B., Shieh M.-S., Solution method for nonlinear problems with multiple critical points, AIAA journal, 1990, vol. 28, p. 2110

Zhang W.-M., Yan H., Peng Z.-K., Meng G., Electrostatic pull-in instability in MEMS/NEMS: A review, Sensors and Actuators A: Physical, 2014, vol. 214, p. 187 
Appendices 



\section{.1 Lei de Material de Kirchhof Saint Venant}

A lei material KSV frequentemente utilizada em análises não-lineares geométrica devida a sua simplicidade, é basicamente uma extensão do modelo linear elástico baseado na lei de Hooke. Utiliza o tensor de deformação de Green $\boldsymbol{E}$, permitindo desta forma obter resultados plausíveis. No entanto é um modelo que não possuí garantia de policonvexidade (ver (Ball, 1976)), ou seja é uma lei material que possui instabilidades quando sujeito a grandes deformações podendo levar a problemas estáticos sem solução. Ainda assim foi muito utilizada nos primeiros artigos de não-linearidade geométrica envolvendo o MOT (Buhl et al., 2000; Gea e Luo, 2001; Lahuerta et al., 2013).

A função da energia de deformação específica da lei de material de KSV é dada por:

$$
\varphi^{0}\left(\mathrm{I}_{1}^{\mathrm{E}}, \mathrm{I}_{2}^{\mathrm{E}}\right)=\frac{\lambda}{2}\left(\mathrm{I}_{1}^{\mathrm{E}}\right)^{2}+\mu \mathrm{I}_{2}=\frac{\lambda}{2}\left(\frac{1}{2} f_{\mathrm{i}} \cdot f_{\mathrm{i}}-3\right)^{2}+\mu \mathrm{I}_{2}^{\mathrm{E}}
$$

onde os invariantes do tensor de Green são dados por $\mathrm{I}_{1}^{\mathrm{E}}$ e $\mathrm{I}_{2}^{\mathrm{E}}$ :

$$
\begin{aligned}
& \mathrm{I}_{1}^{\mathrm{E}}=\operatorname{tr}(\boldsymbol{E})=\boldsymbol{I}: \boldsymbol{E}=\mathrm{E}_{\mathrm{ii}}, \\
& \mathrm{I}_{2}^{\mathrm{E}}=\frac{1}{2}\left((\operatorname{tr} \boldsymbol{E})^{2}-\operatorname{tr} \boldsymbol{E}^{2}\right)=\frac{1}{2}\left(\boldsymbol{E}: \boldsymbol{E}-(\boldsymbol{I}: \boldsymbol{E})^{2}\right) .
\end{aligned}
$$

A primeira variação da função de energia de deformação (.1) em relação ao gradiente da transformação:

$$
\begin{aligned}
\frac{\partial \varphi^{0}}{\partial \boldsymbol{F}} & =\boldsymbol{P}_{0}=\lambda \mathrm{I}_{1}^{\mathrm{E}} \boldsymbol{F}+\mu(\boldsymbol{B}-\boldsymbol{I}) \boldsymbol{F} \\
& =\boldsymbol{\tau}_{i}^{0} \otimes \boldsymbol{e}_{\mathrm{i}}^{\mathrm{r}}=\left(\lambda \mathrm{I}_{1}^{\mathrm{E}}-\mu\right)\left(\boldsymbol{f}_{\mathrm{i}} \otimes \boldsymbol{e}_{\mathrm{i}}^{\mathrm{r}}\right)+\mu\left(f_{j} \otimes \boldsymbol{f}_{j}\right)\left(\boldsymbol{f}_{\mathrm{i}} \otimes \boldsymbol{e}_{\mathrm{i}}^{\mathrm{r}}\right) \\
& =\left(\left(\lambda \mathrm{I}_{1}^{\mathrm{E}}-\mu\right)+\mu\left(\boldsymbol{f}_{j} \otimes f_{j}\right)\right) \boldsymbol{f}_{\mathrm{i}} \otimes \boldsymbol{e}_{\mathrm{i}}^{\mathrm{r}} .
\end{aligned}
$$

\section{.1.1 Tensor Tangente Material}

O tensor tangente material $C_{i j}^{0}$ para a lei material KSV (ver Seção .1) Lahuerta et al. (2013) é obtido através da derivada parcial do tensor $\tau_{i}^{0}$ PK1 (.3) em relação ao gradiente 
dos deslocamentos $\gamma_{j}$ :

$$
\frac{\partial \tau_{i}}{\partial \gamma_{j}}=C_{i j}^{0}=\lambda f_{i} \otimes f_{j}+\mu\left[\left(f_{i} \cdot f_{j}\right) I+f_{j} \otimes f_{i}\right]
$$

Para obter $C_{i j}^{0}$ considerando a hipótese EPD (3.14) matematicamente consistente, antes é necessário igualar as deformações na direção 3 à 0 impondo $\gamma_{33}=0$ no cálculo do tensor das tensões de engenharia $\tau_{i}^{0}(.3)$, obtendo:

$$
\boldsymbol{\tau}_{\alpha}^{0}=\left[\left(\lambda \mathrm{I}_{1}^{(\mathrm{D})}-\mu\right) \boldsymbol{I}+\mu\left(f_{\theta} \otimes f_{\theta}\right)\right] f_{\alpha}
$$

Aplicando a mesma derivação utilizada na equação (.4) no tensor das tensões de engenharia PK1 dado em (.5) é possível obter o tensor tangente material EPD,

$$
\left[\frac{\partial \tau_{\alpha}}{\partial \gamma_{\beta}}\right]^{(\mathrm{D})}=\left[C_{\alpha \beta}^{0}\right]^{(\mathrm{D})}=\lambda f_{\alpha} \otimes f_{\beta}+\mu\left[\left(f_{\alpha} \cdot f_{\beta}\right) \boldsymbol{I}+f_{\beta} \otimes f_{\alpha}\right] .
$$

No caso da hipótese EPT (3.16) para obter o tensor tangente material $C_{\mathrm{ij}}^{0}$ matematicamente consistente é necessário impor $1+\gamma_{33}$ no cálculo do tensor das tensões de engenharia $\tau_{i}^{0}$ e no respectivo invariante $I_{1}$ das deformações de Cauchy-Green, obtendo o tensor das tensões de engenharia:

$$
\left[\boldsymbol{\tau}_{i}^{0}\right]^{(\mathrm{T})}=\left(\lambda \mathrm{I}_{1}^{(\mathrm{D})}-\mu\right) f_{\mathrm{i}}+\mu\left(f_{\gamma} \otimes f_{\gamma}\right) f_{i}+\lambda \tilde{\mathrm{I}}_{1} f_{i}+\mu\left(f_{3} \otimes f_{3}\right) f_{i}
$$

Isolando as variáveis com componentes em 3 na equação (.7) obtemos:

$$
\left[\boldsymbol{\tau}_{i}^{0}\right]^{(T)}=\left[\boldsymbol{\tau}_{i}^{0}\right]^{(D)}+\omega_{\alpha}^{0}
$$

onde $\boldsymbol{\omega}_{\alpha}^{0}$ é dado por:

$$
\boldsymbol{\omega}_{\alpha}^{0}=\frac{\lambda}{2}\left(f_{3} \cdot f_{3}-1\right) f_{\alpha}=\frac{\lambda}{2}\left((1+\gamma)^{2}-1\right) f_{\alpha}
$$

Para obter $\boldsymbol{\omega}_{\alpha}^{0}$ em relação as componentes 1 e 2 do gradiente da transformação nas bases de referencia $\boldsymbol{e}_{\alpha}^{r}$, é necessário encontrar $\gamma_{33}$, no entanto é preciso isolar o termo $\left[\tau_{33}\right]^{(T)}$ do tensor das tensões de engenharia, substituindo a equação (3.16) na equação 
(.7). A partir de $\tau_{3}^{0}$, depois de algumas simplificações obtemos:

$$
\left[\boldsymbol{\tau}_{3}^{0}\right]^{(\mathrm{T})}=\left[\lambda\left(\mathrm{I}_{1}^{(\mathrm{D})}-\frac{1}{2}\right)-\mu\right]\left(1+\gamma_{33}\right) \boldsymbol{e}_{3}^{\mathrm{r}}+\left(\frac{\lambda+2 \mu}{2}\right)\left(1+\gamma_{33}\right)^{3} \boldsymbol{e}_{3}^{\mathrm{r}}
$$

aplicando $\tau_{33}^{0}=\tau_{3}^{0} \cdot \boldsymbol{e}_{3}^{\mathrm{r}}$, projetando na base $\boldsymbol{e}_{3}^{\mathrm{r}}$

$$
\left[\tau_{33}^{0}\right]^{(\mathrm{T})}=\left[\lambda\left(\mathrm{I}_{1}^{(\mathrm{D})}-\frac{1}{2}\right)-\mu\right]\left(1+\gamma_{33}\right)+\left(\frac{\lambda+2 \mu}{2}\right)\left(1+\gamma_{33}\right)^{3} .
$$

Lembrando que para problemas bidimensionais (2D), as componentes da tensão de engenharia $\tau_{\alpha 3}^{0}$ e $\tau_{3 \alpha}^{0}$ são iguais à 0 (zero). Aplicando a hipótese doEPT, onde $\tau_{33}^{0}=0$ na equação (.11), o estiramento $\gamma_{33}$ é obtido:

$$
\gamma_{33}=\sqrt{\frac{\lambda\left(1-2 \mathrm{I}_{1}^{(\mathrm{D})}\right)+2 \mu}{\lambda+2 \mu}}-1
$$

Substituindo $\gamma_{33}$ da equação (.12) no tensor $\boldsymbol{\omega}_{\alpha}^{0}$ dado pela equação (.9) obtemos:

$$
\boldsymbol{\omega}_{\alpha}^{0}=-\frac{\lambda^{2} \mathrm{I}_{1}^{(\mathrm{D})}}{\lambda+2 \mu} f_{\alpha}
$$

Feita todas as deduções e hipóteses prévias relativo ao EPT é possível obter à partir das equações (.6), (.9) e (.13) o tensor tangente material:

$$
\left[C_{\alpha \beta}^{0}\right]^{(\mathrm{T})}=\frac{\partial\left[\boldsymbol{\tau}_{\alpha}^{0}\right]^{(\mathrm{D})}}{\partial \gamma_{\beta}}+\frac{\partial \boldsymbol{\omega}_{\alpha}^{0}}{\partial \gamma_{\beta}}=\left[C_{\alpha \beta}^{0}\right]^{(\mathrm{D})}+Q_{\alpha \beta}^{0},
$$

onde o tensor $Q_{\alpha \beta}^{0}$ é dado por:

$$
Q_{\alpha \beta}^{0}=-\frac{\lambda^{2}}{\lambda+2 \mu}\left(f_{\alpha} \otimes f_{\beta}+\delta_{\alpha \beta} I_{1}^{(D)} I\right)
$$

\section{.2 Projeto de Mecanismo Flexível via MOT}

Nesta Seção é descrito as principais formulações utilizadas na literatura para o projeto ou síntese de mecanismos flexíveis. 


\section{.2.1 Maximização dos Deslocamentos na Porta de Saída}

A maximização do deslocamento na porta de saída de um estrutura impõem na condição de contorno a necessidade de uma força ou deslocamento prescrito na porta de entrada. Neste caso estamos considerando deslocamento prescrito na porta de entrada $\underline{\mathbf{u}}_{\text {in }}$. Logo a formulação geral do problema de otimização é dado por:

$$
\begin{array}{ll}
\operatorname{Min}_{\rho_{\mathrm{i}} \in[0,1]} & \mathbf{u}_{\mathrm{ou}} \\
\text { Sujeito à } & \delta \Pi_{\mathrm{p}}\left(\rho_{\mathrm{i}}, \boldsymbol{u}, \delta \boldsymbol{u}\right)=0 \\
& \overline{\mathrm{V}}^{\mathrm{r}}\left(\rho_{\mathrm{i}}\right)-\underline{\mathrm{v}} \leqslant 0 \\
& \mathbf{u}_{\text {in }}-\underline{\mathbf{u}}_{\text {in }} \leqslant 0 \\
& 0.0 \leqslant \rho_{\mathrm{i}} \leqslant 1.0 \\
& r_{\min } \leqslant r^{*}\left(\Omega_{\mathrm{d}}^{\mathrm{r}}\right)
\end{array}
$$

\section{.2.2 Maximização da Energia de Deformação}

A maximização da energia de deformação de uma estruturas tem pouca aplicação no projeto de estruturas, no entanto sua aplicação no projeto de mecanismos flexíveis tem o objetivo maximizar MA ou GA, irá depender das restrições adicionais impostas, no caso deslocamentos ou forças nas respectivas portas de entrada ou saída. Aqui iremos apresentar uma formulação geral proposta por Cardoso e Fonseca (2004).

$$
\begin{array}{ll}
\operatorname{Min}_{\rho_{\mathrm{i}} \in[0,1]} & \mathrm{u}_{\text {int }} \\
\text { Sujeito à } & \delta \Pi_{\mathrm{p}}\left(\rho_{\mathrm{i}}, \boldsymbol{u}, \delta \boldsymbol{u}\right)=0 \\
& \bar{V}^{\mathrm{r}}\left(\rho_{\mathrm{i}}\right)-\underline{\mathrm{v}} \leqslant 0 \\
& \mathbf{u}_{\text {in }}-\underline{\mathbf{u}}_{\mathrm{in}} \leqslant 0 \\
& \mathbf{u}_{\mathrm{ou}}-\underline{\mathbf{u}}_{\mathrm{ou}} \leqslant 0 \\
& \mathrm{r}_{\min } \leqslant \mathrm{r}^{*}\left(\Omega_{\mathrm{d}}^{\mathrm{r}}\right)
\end{array}
$$

As restrições de desigualdade de projeto são dadas por (ํ)), portanto nesta formulação são impostas restrição de deslocamento nas portas de entrada saída, ou seja uma restrição 
de GA (4.28) é imposta. Com a maximização da energia de deformação então busca-se minimizar a energia retida pelo mecanismo $(U)$ dada pela equação (4.26) respectivamente.

\section{.3 Métodos Numéricos de Otimização}

Nesta seção são apresentadas a formulação básica do Critério da Optimalidade (ㅈ) e MAM, que são métodos de otimização numéricos utilizados em larga escala no MOT, portanto iremos demonstrar que o MAM é uma método basado no $\overline{C O}$ assim como o CONLIN

\subsection{Método do Critério da Optimalidade}

O método do CO é baseado em algoritmos de atualização das variáveis de projeto que se baseiam na condição de estacionariedade da função Lagrangiana do problema a ser minimizado (Haftka e Gürdal, 2012), estimando as variáveis duais através o método da bisseção, quando este atinge a fronteira da restrição, obtendo assim grande eficiência numérica. Este método opera de forma similar ao método de PLS, pois se baseiam na solução de uma sequencia de aproximações de primeira ordem do problema (primeira derivada) para solucionar um modelo não-linear. OCO é um método muito específico, pois geralmente as restrições são lineares e muitas restrições de projeto podem tornar a solução de ${ }^{*} \rho_{i},{ }^{*} \Lambda_{j}$ muito custosa, pois as variáveis duais são estimadas. Um vantagem também está relacionado ao valor inicial das variáveis, pois a regra de atualização das variáveis duais $\left(\Lambda_{j}\right)$ forçam resultados somente dentro do domínio viável. Na literatura é muito discutida a origem do CO, e muitos afirmam ser um Método Empírico ou sem origem matemática (Groenwold e Etman, 2008), no entanto iremos demonstrar que estas afirmações não se sustentam e que o método tem uma origem na condição de estacionariedade da função Lagrangiana (3.55) utilizando aproximações locais lineares e reciprocas (Starnes Ir e Haftka, 1979). Uma função local pode ser aproximada 
linearmente utilizando o termo da derivada de primeira da série de Taylor dada por:

$$
\bar{g}_{\mathrm{L}}=g^{(0)}-g_{, \mathrm{i}}^{(0)}\left(\rho_{\mathrm{i}}-\rho_{\mathrm{i}}^{(0)}\right), \quad g_{, \mathrm{i}}^{(0)}=\left.\frac{\partial g}{\partial \rho_{\mathrm{i}}}\right|_{\rho_{\mathrm{i} 0}},
$$

da mesma forma pode ser aproximada por uma função que é linearmente inversa em relação as variáveis de projeto, também chamada de aproximação reciproca:

$$
\bar{g}_{\mathrm{R}}=g^{(0)}-g_{, i}^{(0)}\left(\frac{\rho_{i}^{(0)}}{\rho_{i}}\right)\left(\rho_{i}-\rho_{i}^{(0)}\right) .
$$

A combinação das duas aproximações (.18) e (.19) obtemos uma função denominada de aproximação hibrida (Starnes Ir e Haftka, 1979) ou aproximação linear convexa (Fleurv, 1989):

$$
\bar{g}_{\mathrm{H}}=g^{(0)}-g_{, \mathrm{i}}^{(0)} v_{\mathrm{i}}
$$

onde o termo $v_{i}$ depende do sinal da derivada da função a ser aproximada:

$$
v_{i}=\left\{\begin{array}{ll}
\left(\rho_{i}-\rho_{i}^{(0)}\right) & \text { se } g_{, i}^{(0)}<0 \\
\left(\frac{\rho_{i}^{(0)}}{\rho_{i}}\right)\left(\rho_{i}-\rho_{i}^{(0)}\right), & \text { se } g_{, i}^{(0)} \geqslant 0
\end{array} .\right.
$$

Esta funcão de aproximacão hibrida (.20) foi inicialmente proposta por Starnes Jr e Haftka (1979) e precursora do método denominado por Convex Linearization (CONLIN) (Fleury, 1989) e sua modificação por assintotas (Svanberg, 1987) gerou o MAM que será descrito na Seção 3.2 em detalhes. No caso do problema de minimização da flexibilidade, com restrição de volume, proposto inicialmente por Bendsøe e Kikuchi (1988), foi avaliado que a derivada da função de flexibilidade em relação as variáveis de projeto possui valores negativos e a função de volume valores positivos, portanto a aproximação da função objetivo $f_{0}$ deve ser aproximada por uma função não-linear, no caso uma aproximação reciproca (.19) e a função de restrição $f_{j}$ por uma função linear 
(.18) na condição de estacionariedade da função Lagrangiana (3.55) na iteração (k):

$$
\begin{aligned}
\frac{\partial \mathcal{L}\left(\rho_{i}\right)}{\partial \rho_{i}} & =\frac{\partial\left[\bar{f}_{\mathrm{R}}\right]_{0}^{(k)}}{\partial \rho_{i}}+\frac{\partial}{\partial \rho_{i}}\left(\sum_{j=1}^{n_{g}} \Lambda_{j}\left[\bar{f}_{\mathrm{L}}\right]_{j}^{(k)}\right) \\
& =f_{0, i}^{(k)}+\sum_{j=1}^{n_{g}} \Lambda_{j} f_{j, i}^{(k)}\left(\frac{\rho_{i}^{(k)}}{\rho_{i}}\right)^{2}=0 .
\end{aligned}
$$

Isolando o termo $\rho_{i}$ de (.22), obtemos a solução do problema relativo as variáveis primais:

$$
\begin{aligned}
\rho_{i}^{(k+1)} & =\rho_{i}^{(k)}\left(\frac{f_{0, i}^{(k)}}{\sum_{j=1}^{n_{g}} \Lambda_{j} f_{j, i}^{(k)}}\right)^{1 / 2} \\
& =\rho_{i}^{(k)} B_{i}^{k}
\end{aligned}
$$

onde o parâmetro $\kappa$ se refere a uma constante exponencial dada pela combinação entre a função de aproximação linear na função objetivo e uma função de aproximação reciproca nas funções de restrições. No entanto, na literatura (Bendsøe, 1995; Groenwold e Etman, 2008) o parâmetro k é descrito como um parâmetro de amortecimento heurístico que tem a função de melhorar a convergência e a estabilidade do processo iterativo do CO, mas como demonstrado em (.22) e (.23), este parâmetro tem o valor de "1/2"não por acaso, logo não pode-se afirmar que se trata de um parâmetro heurístico. Por outro lado, sua alteração tem sim uma conotação heurística já que não se tem prova ou demonstração matemática que justifiquem sua alteração, somente testes numéricos demonstraram um retardo no processo de convergência, sendo o mesmo efeito obtido por uma alteração nos parâmetros $\left(\alpha_{i}, \beta_{i}\right)$ na função do limite móvel (.25).

Em relação as variáveis primais $\rho_{i}^{(k+1)}$ é necessário impor limites móveis como restrições de caixa a cada iteração (k) com um limite superior $\underline{\rho}_{i}^{(k)}$ e inferior $\bar{\rho}_{i}^{(k)}$ de cada variável da seguinte forma:

$$
\rho_{i}^{(k+1)}=\left\{\begin{array}{lll}
\rho_{i}^{(k)} B_{i}^{1 / 2} & \text { se } & \underline{\rho}_{i}^{(k)}<\rho_{i}^{(k)} B_{i}^{1 / 2}<\bar{\rho}_{i} \\
\underline{\rho}_{i}^{(k)} & \text { se } & \rho_{i}^{(k)} B_{i}^{1 / 2} \leqslant \underline{\rho}_{i}^{(k)} \\
\bar{\rho}_{i}^{(k)} & \text { se } & \rho_{i}^{(k)} B_{i}^{1 / 2} \geqslant \bar{\rho}_{i}^{(k)}
\end{array},\right.
$$


também é necessário impor as restrições de caixa no processo global de otimização, dada por:

$$
\begin{aligned}
& \underline{\rho}_{i}^{(k)} \leftarrow \max \left(\rho_{i}^{(k)}-\alpha_{i}, \rho_{\text {min }}\right), \\
& \bar{\rho}_{i}^{(k)} \leftarrow \min \left(\rho_{i}^{(k)}+\beta_{i}, \rho_{\text {max }}\right) .
\end{aligned}
$$

Todo este processo de otimização é dependente das variáveis duais para obter a contra parte primal das variáveis dada pela equação (.23). Para resolver este problema o método de bisseção é utilizado para estimar as variáveis $\Lambda_{j}$, ou seja cada iteração $(k)$ cria uma sub-problema com um looping que só é interrompido quando a verificação da função de restrição estiver dentro ou na fronteira do domínio viável $\left(f_{j}\left(\rho_{i}\right) \leqslant b_{i}\right)$.

Finalmente a convergência do método do COé dada pela pelo seguinte comparação entre entre as variáveis:

$$
\left\|\rho_{i}^{(k+1)}-\rho_{i}^{(k)}\right\|_{2}<\varepsilon,
$$

onde $\varepsilon$ é uma tolerância mínima admitida na verificação da convergência.

\section{.3.2 Método das Assíntotas Móveis}

O MAM foi originalmente proposto por Svanberg (1987) como um novo método de otimização para a solução de problemas não-lineares, este método opera de forma muito similar aos métodos do $\mathrm{CO}$ e ao CONLIN pois resolve uma sequência de aproximações mais simples do problema original (com uma função convexa separável) utilizando uma linearização da aproximação onde é possível ajustar o grau de convexidade e conservatividade do problema. Esta função de aproximação é dada por:

$$
\bar{g}_{A}=r^{(k)}+\left(\frac{p_{i}^{(k)}}{U_{i}^{(k)}-\rho_{i}}+\frac{q_{i}^{(k)}}{\rho_{i}-L_{i}^{(k)}}\right)
$$

onde $U_{i}$ e $L_{i}$ são referentes aos parâmetros de ajuste da função de aproximação denominados de assintotas móveis superior e inferior dada na iteração $(k)$ respectivamente. 
Os parâmetros $r^{(k)}, p_{i}^{(k)}$ e $q_{i}^{(k)}$ são definidos por:

$$
\begin{aligned}
& p_{i}^{(k+1)}= \begin{cases}\left(u_{i}^{(k)}-\rho_{i}^{(k)}\right)^{2} f_{, i}^{(k)} & \text { se } f_{, i}^{(k)}>0 \\
0, & \text { se } f_{, i}^{(k)} \leqslant 0\end{cases} \\
& q_{i}^{(k+1)}= \begin{cases}0, & \text { se } f_{, i}^{(k)} \geqslant 0 \\
-\left(\rho_{i}^{(k)}-L_{i}^{(k)}\right)^{2} f_{, i}^{(k)} & \text { se } f_{, i}^{(k)}<0\end{cases} \\
& r^{(k)}=f^{(k)}-\left(\frac{p_{i}^{(k)}}{u_{i}^{(k)}-\rho_{i}^{(k)}}+\frac{q_{i}^{(k)}}{\rho_{i}^{(k)}-L_{i}^{(k)}}\right) .
\end{aligned}
$$

Observando as equações (.20) e (.21) que definem a aproximação híbrida e a aproximação por assintotas móveis dada pelas equações $(.20),(.20),(.20)$ e $(.20)$ podemos verificar a separação das funções de aproximação baseada no sinal dos gradientes da primeira derivada e desta forma concluir que o MAMé um método derivado doCO. Também foi observado por Groenwold e Etman (2008) através experimentos numéricos a semelhança entre os métodos e constatou que os resultados eram muito parecidos ou quase idênticos para alguns exemplos e muito próximo para os outros, porém com uma convergência mais lenta. Conforme Svanberg (1987), os valores das assintotas móveis tem a seguinte banda de valores em relação a variável de projeto:

$$
-\infty<\mathrm{L}_{i}^{(\mathrm{k})}<\rho_{i}^{(\mathrm{k})}<\mathrm{U}_{i}^{(\mathrm{k})}<+\infty
$$

portando se aplicarmos os valores de $\mathrm{L}_{\mathrm{i}}^{(\mathrm{k})}=0$ e $\mathrm{U}_{\mathrm{i}}^{(\mathrm{k})}=+\infty$ na função de aproximação (.27) obtemos uma função de aproximação hibrida conforme descrita na equação (.21) do CO, corroborando com trabalho de Groenwold e Etman (2008).

As derivadas de segunda ordem da função de aproximação (.27) podem ser utilizadas pelo MAM para dar mais estabilidade no processo de otimização e são apresentadas por:

$$
\frac{\partial^{2} f_{A}^{(k)}}{\partial \rho_{i}^{2}}=\frac{2 p_{i}^{(k)}}{\left(u_{i}^{(k)}-\rho_{i}^{(k)}\right)^{3}}+\frac{2 q_{i}^{(k)}}{\left(\rho_{i}^{(k)}-L_{i}^{(k)}\right)^{3}}, \quad \frac{\partial^{2} f^{(k)}}{\partial \rho_{i} \partial \rho_{l}}=0, \text { se } i \neq l .
$$


Lembrando que para $\mathrm{p}_{i}^{(\mathrm{k})} \geqslant 0$ e $\mathrm{q}_{i}^{(\mathrm{k})} \geqslant 0$, a função $f^{(\mathrm{k})}$ é convexa e portanto:

$$
\frac{\partial^{2} f^{(k)}}{\partial \rho_{i}^{2}}= \begin{cases}\frac{2 f_{, i}^{(k)}}{\left(u_{i}^{(k)}-\rho_{i}^{(k)}\right)}, & \text { se } f_{, i}^{(k)}>0, \\ -\frac{2 f_{, i}^{(k)}}{\left(\rho_{i}^{(k)}-L_{i}^{(k)}\right)}, & \text { se } f_{, i}^{(k)}<0 .\end{cases}
$$

Consequentemente quanto mais próximo os valores das assintotas móveis de $\mathrm{L}_{i}^{(k)}$ e $u_{i}^{(k)}$ das variáveis de projeto $x_{i}^{(k)}$, maior será os valores das derivadas de segunda ordem, maior será a curvatura da função de aproximação $f_{j}^{(k)}$ e mais conservativa será a aproximação do problema, deste modo mais precisa será a solução (Svanberg, 1987).

A estratégia adaptativa dos limites moveis leva em conta a atualização das assintotas móveis, considerando uma regra heurística de atualização em relação a iteração:

- $\operatorname{Para}(k) \leqslant 1$ :

$$
\mathrm{L}_{i}^{(\mathrm{k})}=\rho_{i}^{(\mathrm{k})}-\left(\bar{\rho}_{i}^{(\mathrm{k})}-\underline{\rho}_{i}^{(\mathrm{k})}\right), \quad \mathrm{U}_{i}^{(\mathrm{k})}=\rho_{i}^{(\mathrm{k})}+\left(\bar{\rho}_{i}^{(\mathrm{k})}-\underline{\rho}_{i}^{(\mathrm{k})}\right),
$$

onde o limite superior $\underline{\rho}_{i}^{(k)}$ e inferior $\bar{\rho}_{i}^{(k)}$ das variáveis primais utilizam a mesma regra das restrições de caixa dada pela equação (.25).

- $\operatorname{Para}(k) \geqslant 2$ :

Se $\operatorname{sign}\left(\rho_{i}^{(k)}-\rho_{i}^{(k-1)}\right) \neq\left(\rho_{i}^{(k-1)}-\rho_{i}^{(k-2)}\right)$, indica que o processo iterativo de otimização está oscilando, então as assintotas móveis devem ter a seguinte regra de atualização:

$$
\begin{aligned}
& \mathrm{L}_{i}^{(\mathrm{k})}=\rho_{i}^{(\mathrm{k})}-s\left(\rho_{i}^{(\mathrm{k}-1)}-\mathrm{L}_{i}^{(\mathrm{k}-1)}\right), \\
& \mathrm{u}_{i}^{(\mathrm{k})}=\rho_{i}^{(\mathrm{k})}+s\left(\mathrm{U}_{i}^{(\mathrm{k}-1)}-\rho_{i}^{(k-1)}\right),
\end{aligned}
$$

onde s é uma fator de atualização empírico das assintotas móveis, descrito no artigo de Svanberg (1987) como s $=0.7$. 
$\operatorname{Se} \operatorname{sign}\left(\rho_{i}^{(k)}-\rho_{i}^{(k-1)}\right)=\left(\rho_{i}^{(k-1)}-\rho_{i}^{(k-2)}\right)$, ou seja tem o mesmo sinal, indica que as assintotas estão convergindo e a regra de atualização é dada por:

$$
\begin{aligned}
& \mathrm{L}_{i}^{(\mathrm{k})}=\rho_{i}^{(\mathrm{k})}-\left(\rho_{i}^{(\mathrm{k}-1)}-\mathrm{L}_{\mathrm{i}}^{(\mathrm{k}-1)}\right) / \mathrm{s}, \\
& \mathrm{u}_{i}^{(\mathrm{k})}=\rho_{i}^{(\mathrm{k})}+\left(\mathrm{U}_{i}^{(\mathrm{k}-1)}-\rho_{i}^{(\mathrm{k}-1)}\right) / s,
\end{aligned}
$$

Logo podemos descrever de forma generalizada a relação entre as assintotas móveis e os limites móveis:

$$
\mathrm{L}_{i}^{(\mathrm{k})}<\alpha_{i}^{(\mathrm{k})}<\rho_{i}^{(\mathrm{k})}<\beta_{i}^{(\mathrm{k})}<\mathrm{U}_{i}^{(\mathrm{k})}
$$

Como é possível concluir ao longo deste Apêndice que o MAMé um método derivado do CO, pois compartilha a mesma dependência das variáveis duais (Groenwold e Etman, 2008), logo é possível obter a relação entre as variáveis primais e duais aplicando a função de aproximação (.27) sujeita a condição de estacionariedade (3.55) dada por:

$$
\rho_{i}=\frac{\left(p_{0 i}+\sum_{j=1}^{n_{g}} \Lambda_{j} f_{j, i}\right)^{1 / 2} L_{i}+\left(q_{0 i}+\sum_{j=1}^{n_{g}} \Lambda_{j} f_{j, i}\right)^{1 / 2} u_{i}}{\left(p_{0 i}+\sum_{j=1}^{n_{g}} \Lambda_{j} f_{j, i}\right)^{1 / 2}+\left(q_{0 i}+\sum_{j=1}^{n_{g}} \Lambda_{j} f_{j, i}\right)^{1 / 2}}
$$

